
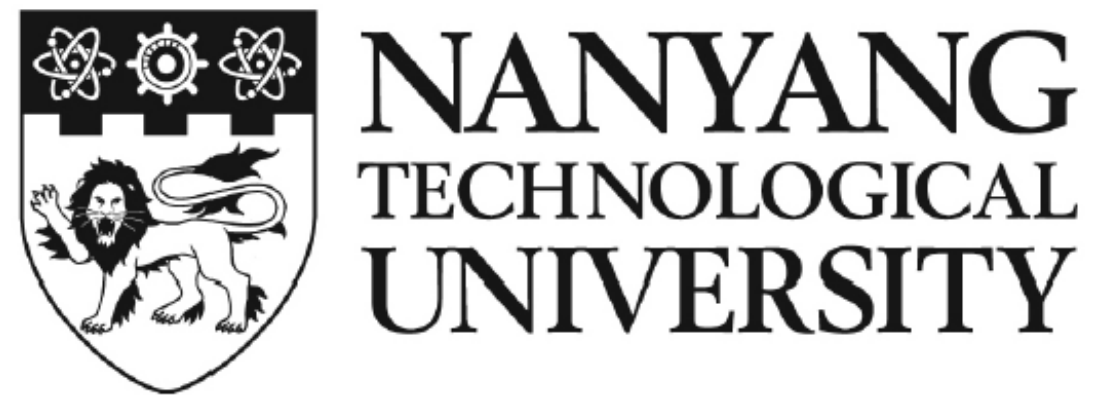

\title{
LARGE-SCALE CAPACITIVE TOUCH PANELS: SENSOR PATTERN DESIGN, SAMPLING AND INTERPOLATION
}

\author{
HUMZA AKHTAR
}

SCHOOL OF COMPUTER SCIENCE AND ENGINEERING 


\section{LARGE-SCALE CAPACITIVE TOUCH PANELS: SENSOR PATTERN DESIGN, SAMPLING AND \\ INTERPOLATION}

\section{HUMZA AKHTAR}

\section{SCHOOL OF COMPUTER SCIENCE AND ENGINEERING}

A thesis submitted to the Nanyang Technological University in partial fulfilment of the requirement for the degree of

Doctor of Philosophy 
To my wife, Mahham, who has always been a beacon of light for me in dark times during my research. 


\section{Acknowledgments}

I would like to express my sincere gratitude to my supervisor, Prof. Kemao Qian and my ex-supervisor Prof. Ramakrishna Kakarala for their continued support and guidance since I started my $\mathrm{PhD}$ in 2012. I am thankful to them for the amount of independence they gave me during my $\mathrm{PhD}$, which allowed me to start thinking as a researcher. I would also like to thank them for the confidence they showed in me and the patience that they had for me when I was busy with my full time job. I thank them for always keeping their door open for me to discuss my research problems, even at odd times during the day. Finally, I am grateful for the support and motivation that they have provided during the course of my $\mathrm{PhD}$.

I would like to say thanks to Earth Observatory of Singapore, NTU and in particular Prof. Kerry Sieh, Prof. Isaac Kerlow and Dr. Susan Eriksson for financially supporting my PhD during the last four and a half years. It was hard to work as a full time employee and at the same time do my $\mathrm{PhD}$ and I could not have done it without their understanding and generous support.

Finally, I would like to express my gratefulness to my parents, Dr. Tanvir Akhtar and Shagufta Yasmeen and my sister Rumma for helping me realize my own potential. All the support they have provided me over the years was the greatest gift anyone has ever given me. Without them, I may never have gotten to where I am today. 


\section{Abstract}

A touch panel is an input device for human computer interaction. It consists of a network of sensors, a sampling circuit and a controller for detecting and locating a touch input. Touch input can come from either finger or stylus depending upon the type of touch technology. These touch panels provide an intuitive and collaborative workspace so that people can perform various tasks without the use of traditional input devices like keyboard and mouse, and with the use of their fingers. Our research studies and tries to improve upon the traditional design and algorithms for capacitive touch technology.

We begin by briefly describing the types of touch technology and observe that according to market research, capacitive touch will dominate the touch panel industry in years to come. We differentiate between touch panels and touch screens based on size, and steer the direction of this thesis towards touch panels after identifying three unanswered research questions that come up while designing large-scale capacitive touch panels. We discuss role of large-scale touch panels in Earth science education and explain the motivation for doing research on large-scale panels.

The first question asks about the best geometrical design for a capacitive touch sensor. Capacitive sensors are arranged on a touch panel, whether it is a smart phone or a wall mount display in a grid formation. Industries use their own patented designs for manufacturing grid patterns and the efficiency of a touch panel relies on the geometry and arrangement of sensors on the grid. We study various designs patented by industry over the last few years and propose a methodology for testing and comparing different geometrical designs in a simulation environment. Such 
methodology was not explored in academic literature at the time of this research and it opened up an avenue for further work by other researchers in this field. By treating touch panels as binary images, we study their Fourier transform and create a hybrid sensor by combining magnitude and phase of two different designs. We also propose geometrical changes to increase the sensing area of the existing designs and test the sensitivity of sensor patterns using ANSYS Method Of Moments (MOM) capacitance solver.

The second question deals with the transformation of small-scale touch panel to a large-scale one. We look at the sampling circuit of a traditional capacitive touch panel and identify that for large panels, efficient sampling is required in order to avoid latency. Considering compressive sensing as the answer to our sampling problem, we propose algorithms which use structured binary matrices for an efficient touch signal recovery, and show their implementation and memory constraints. It is also shown that such algorithms under-perform in the presence of noise. Looking at the capacitive touch sampling problem carefully, one realizes that the touch signal is highly structured and therefore we propose modified greedy algorithms for capacitive touch sampling which are not only efficient, but accurate in the presence of noise. This work further bridges the gap between two independent but actively researched fields, that is, compressive sensing and capacitive touch sensing.

The third and the last question is related to subpixel interpolation procedure at the touch controller. Industry is using simple three-point interpolation schemes but as is the case with sensor design, no one has explored the possibility for an efficient interpolation algorithm designed specific to capacitive touch signals. We apply various one dimensional, two dimensional and iterative subpixel interpolators for the case of a human finger covering more than one sensor in both row and column direction while touching the panel, and compare them with each other in terms of efficiency and accuracy. Finally, at the end, we propose a full framework for capacitive touch panel including an improved sensor, an efficient sampling algorithm and an accurate subpixel interpolator. 


\section{Contents}

Acknowledgments $\quad$ i

$\begin{array}{ll}\text { Abstract } & \text { ii }\end{array}$

List of Contents $\quad$ iv

List of Figures viii

List of Tables $\quad$ xvii

List of Abbreviations $\quad$ xviii

1 Introduction $\quad 1$

1.1 Touch Panel Market Share and Forecast . . . . . . . . . . . . . . . . 3

1.2 Touch Technologies Overview . . . . . . . . . . . . . . . 6

1.2.1 Resistive-based Technology . . . . . . . . . . . . . . 6

1.2.2 Surface Acoustic Wave-based technology . . . . . . . . . . . 8

1.2.3 Infrared-based technology . . . . . . . . . . . . . . . . 10

1.2.4 Surface Capacitive-based technology . . . . . . . . . . . . 11

1.2.5 Projected Capacitive-based technology . . . . . . . . . . . . 12

1.3 Motivation . . . . . . . . . . . . . . . . . . . 19

1.3.1 Issues to Address . . . . . . . . . . . . . . . . . . . . . 21

1.4 Outline and Contribution . . . . . . . . . . . . . . . . . 22 
2.1 Introduction . . . . . . . . . . . . . . . . . 25

2.2 EOS-GeoTouch: Basic Architecture . . . . . . . . . . . . . . . . 28

2.3 EOS-GeoTouch as an Education and Outreach Tool . . . . . . . . . . 31

2.3.1 Quantitative Analysis of EOS-GeoTouch Impact as an Outreach Tool . . . . . . . . . . . . . . . . . 34

2.4 EOS-GeoTouch and Urban Planning . . . . . . . . . . . . . . . 37

2.5 Shortcomings of Infrared Touch Technology and Comparison with Capacitive Touch Technology . . . . . . . . . . . . . . . 38

3 Grid Pattern Design and Testing $\quad 42$

3.1 Introduction and Related Work . . . . . . . . . . . . . . . . . . 42

3.1.1 Touch Panel Stack . . . . . . . . . . . . . . . . . . . . . 42

3.1 .2 Touch Panel Sensor Pattern Design . . . . . . . . . . . . . 44

3.2 Sensor Pattern Testing Methodology . . . . . . . . . . . . . . . . . . 49

3.2.1 Diagonal Movement . . . . . . . . . . . . . . . . . 51

3.2 .2 Circular Movement . . . . . . . . . . . . . . . . 52

3.2.3 Angle Sweep Movement . . . . . . . . . . . . . . . . 56

3.2.4 Tracking Error Measurement Apparatus for Grid Patterns . . 58

3.3 Designing Hybrid Patterns using Fourier Transform . . . . . . . . . . 61

3.4 Designing Efficient Patterns by doing Geometric Changes . . . . . . . 64

3.5 Measuring Sensitivity of Capacitive Pattern using ANSYS Capacitance Solver . . . . . . . . . . . . . . . . 66 66

3.6 Validation of Proposed Methodology . . . . . . . . . . . . . . . 70

3.7 Conclusion and Future Work . . . . . . . . . . . . . . . . . 72 
4.1 Introduction and Related Work . . . . . . . . . . . . . . . . . . 74

4.1.1 Traditional Sampling Techniques . . . . . . . . . . 75

4.1.2 Related Work in Efficient Sampling for Capacitive Touch Panels 80

4.1.3 Compressive Sensing for Capacitive Touch Panels . . . . . . . 82

4.2 Structured Binary Matrices for Sparse Signal Recovery . . . . . . . . 86

4.2.1 Restricted Isometry Property . . . . . . . . . . . 86

4.2.2 Compressive Sensing for Augmented Identity Matrices (CSAIM) 87

4.2.3 Block Compressive Sensing using Binary Matrices (BCSBM) 92

4.3 Greedy Algorithms for Sparse Signal Recovery . . . . . . . . . . . . . 96

4.3.1 Gramian Matching Pursuit (GMP) . . . . . . . . . . . 96

4.3.2 Block Matching Pursuit with Non-Iterative Least Squares (BMPNILS) . . . . . . . . . . . . . . . 101

4.4 Simulations and Results . . . . . . . . . . . . . . . . 110

4.4.1 Sampling Matrix Selection . . . . . . . . . . . . . . . 111

4.4 .2 Recovery Analysis . . . . . . . . . . . . . . . . . . 112

4.4 .3 FLOPS Analysis . . . . . . . . . . . . . . 116

4.4.4 Implementation Analysis . . . . . . . . . . . . . . . 120

4.5 Validation of Algorithm Results . . . . . . . . . . . . . . . . 122

4.6 Conclusion and Future Work . . . . . . . . . . . . . . . . . . . . . 124 
5 Subpixel Interpolation

5.1 Introduction . . . . . . . . . . . . . . . . . . . . 125

5.2 Resolution of Capacitive Touch Panels . . . . . . . . . . . . . . 128

5.2.1 Finger Modeling for Capacitive Touch Panels . . . . . . . . 130

5.3 One Dimensional Three-Point Interpolators . . . . . . . . . . . . . . . 131

5.3.1 Diagonal Movement . . . . . . . . . . . . . 133

5.3 .2 Circular Movement . . . . . . . . . . . . . . 137

5.3.3 Angle Sweep Movement . . . . . . . . . . . . . . . 138

5.3.4 Horizontal,Vertical Movement and Bias of Estimators . . . . . 140

5.3.5 Edge Effect . . . . . . . . . . . . . . . . . 141

$5.4 \quad$ 2-D Gaussian Subpixel Interpolator . . . . . . . . . . . . . . . . . 142

5.5 Simulations and Comparison . . . . . . . . . . . . . . 144

5.6 Validation of Research Findings . . . . . . . . . . . . . . . . . 145

5.7 Conclusion and Future Work . . . . . . . . . . . . . . . . . . . 147

6 Summary and Outlook 149

6.1 Summary . . . . . . . . . . . . . . . . . . . . . . . . . 149

6.1.1 Motivation and Objectives . . . . . . . . . . . . 149

6.1.2 Sensor Pattern Design and Testing . . . . . . . . . . . 150

6.1.3 Efficient and Accurate Sampling . . . . . . . . . . . . 150

6.1 .4 Subpixel Interpolation _. . . . . . . . . . . . . . . 152

6.2 Future Work . . . . . . . . . . . . . . . . . . . 152

6.2.1 Sampling Matrix Training . . . . . . . . . . . . 153

6.2.2 Image Processing Techniques . . . . . . . . . . . . . 153

6.2.3 Accurate Noise Modeling . . . . . . . . . . . . . . . 154

6.3 Final Thoughts . . . . . . . . . . . . . . . . . 155 
List of Publications 


\section{List of Figures}

1.1 Touch panel supply chain comprises of Original Equipment Manufacturer (OEM), Original Design Manufacturer (ODM) and Contract Manufacturers (CMs) supplying various components of the touch panel to the retail market. . . . . . . . . . . . . . . . . 3

1.2 Global retail market touch panel shipment forecast 2014-2019 (million units) $[1] . \ldots \ldots \ldots$

1.3 Touch technologies classification $[2] \ldots \ldots \ldots \ldots$

1.4 Touch panel market share in global retail market by technology in 2014 [1] (figure best viewed in color) . . . . . . . . . . . . 5

1.5 Forecast - Touch panel market share in global retail market by technology in 2019. [1] (figure best viewed in color). . . . . . . . . . . . 6

1.6 In a basic resistive touch screen, the pressure of the finger is used to create electrical contact between the two conductive layers causing a voltage divider circuit to be formed. Source: [3]. . . . . . . . . . . . . 7

1.7 In an AMR touch sensor, the conductive layers are shaped as strips such that each intersection of strips forms a square, Each square is an independent 4 -wire analog touch sensor. Source: [3] . . . . . . . . 8

1.8 Multi-touch system based on SAW technology. In the figure, touch causes the wave to be absorbed causing a delay on its way to the receiving transducers. This disturbance can be picked up by the controller as a possible touch point. Source: [3] . . . . . . . . . . . 9 
1.9 Phenomenon of infrared sensing. Blue and green rectangles represent the photo-receptors and the LEDs respectively. Touching the screen causes a break in the light path as shown by red arrows. Figure reproduced with changes from [2] and is best viewed in color. . . . . . 11

1.10 Working of a surface capacitance based touch panel. . . . . . . . . . . 12

1.11 E. A. Johnson with his invention in 1967 [4]. . . . . . . . . . . . . . . 13

1.12 Phenomenon of capacitive sensing. Blue line represents a capacitive circuit formed between finger and copper pad. $\mathrm{C}_{\mathrm{F}}$ and $\mathrm{C}_{\mathrm{S}}$ represent the finger and sensor capacitance respectively whereas d represents the distance between the plates. Figure reproduced with changes from $[5] \ldots \ldots \ldots \ldots \ldots \ldots$

1.13 Figure depicting difference between self and mutual capacitance. (a) Mutual capacitance (b) Self capacitance. . . . . . . . . . . . . . . 17

1.14 Self capacitance ghost point problem. A self capacitance system will not be able to differentiate between two finger touch $[(x 1, y 2),(x 2, y 1)]$ and $[(x 1, y 1),(x 2, y 2)] \ldots \ldots \ldots \ldots$

1.15 Three main components of a capacitive touch system . . . . . . . . . 19

1.16 Capacitance electrode scaling techniques. (a) Scaling up by keeping electrode size same (b) Scaling up by increasing electrode size. . . . . 20

2.1 Basic components of an individual EOS-GeoTouch system. A large scale multi-touch overlay, a PC running the software and local map server and/or a web map server . . . . . . . . . . . . . . . . . . 29

2.2 (a) The original EOS-GeoTouch just after being installed in EOS lobby (2009). (b) Using GeoTouch to give a presentation on climate change to minister for education and Prime Minister of Singapore

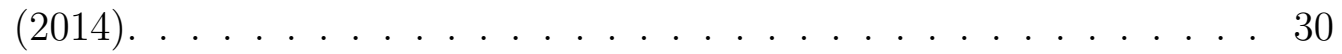

2.3 Basic Concept of a Model-View-View-Model System. . . . . . . . . . 30 
2.4 Simplistic software architecture of EOS-GeoTouch . . . . . . . . . 31

2.5 The main display of EOS-GeoTouch when turned on. . . . . . . . . . 32

2.6 (a) Tools are on the left and (b) Data Layers are on the right hand side of the screen. . . . . . . . . . . . . . . . . . . . 32

2.7 Different features of EOS-GeoTouch. . . . . . . . . . . . . . . 33

2.8 Pie chart representing the breakdown by categories of events in and outside EOS that used the EOS-GeoTouch system (Category A: 25\%, Category B: $33 \%$ and Category C: 42\%) (best viewed in color). . . . 35

2.9 Pie chart representing the breakdown of events by audience type. (Events involving students: $28 \%$, events involving visitors with science background: $28 \%$ and events involving visitors with non scientific background: 44\%) (best viewed in color) . . . . . . . . . . . . 36

2.10 Chart representing number of events per year (2012 - July 2016) . . . 37

2.11 Pie chart showing the percentage of non scientific visitors who interacted with EOS-GeoTouch after an event (61\%) (figure best viewed in Color $\ldots \ldots \ldots \ldots$. . . . . . . . . . . . . . 37

2.12 EOS-GeoTouch and urban planning using the multilayer visualization

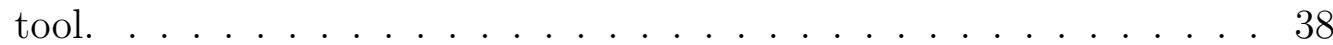

2.13 EOS-GeoTouch touch screen analysis results. The zoomed in shot (b) shows that the finger tracking accuracy is off by some margin. . . . . 40

2.14 Peau Productions infrared touch panel ambient light tests. Five fingers were moved in a straight horizontal line from right to left. The touch points reported are highlighted in red. Screen grabs from [6].

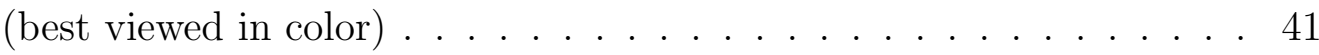

3.1 Typical stack for a touch enabled panel. Figure reproduced from [7] with changes. . . . . . . . . . . . . . . . . 43 
3.2 (a) Sensor on lens stack (b) On-cell stack. Figure reproduced from [7] with changes. . . . . . . . . . . . . . . . . 44

3.3 Diamond shaped (a) basic element and (b) grid pattern for capacitive touch panels. . . . . . . . . . . . . . . . . . . 46

3.4 Grid basic elements (a)-(f) and their respective patterns (g)-(l) formed by replicating the basic element in horizontal and vertical direction. . 48

3.5 (a) Simulated finger profile used in the simulations (b) Finger superimposed on diamond grid. The columns and rows covered by the round finger are highlighted. . . . . . . . . . . . . . . 50

3.6 (a) Touch controller readings with zero noise (b) Touch controller readings with added noise. . . . . . . . . . . . . . 51

3.7 (a) Diagonal movement tracking error in $\mathrm{mm}$ with finger radius $7.5 \mathrm{~mm}$ and no noise (b) with additional noise (c) Finger radius $5 \mathrm{~mm}$ with zero noise $[$ In color $] \ldots \ldots \ldots \ldots$

3.8 A $7.5 \mathrm{~mm}$ radius finger moved in a diagonal path with uniformly distributed random noise of 0.3 amplitude for different grid patterns. The units for rows and columns are in pixels. Blue: Exact Route, Red: Estimated Route. (100 pixels $=5 \mathrm{~mm}$ ). Figure is best viewed in color. . . . . . . . . . . . . . . . . . . . . 54

3.9 A $7.5 \mathrm{~mm}$ radius finger moved in a circular path for different grid patterns. The units for rows and columns are in pixels. Blue: Exact Route, Red: Estimated Route. (100 pixels $=5 \mathrm{~mm})$. Figure is best viewed in color. . . . . . . . . . . . . . . . . . . 55

3.10 Angle sweep performed by a $7.5 \mathrm{~mm}$ radius finger for different grid patterns. The units for rows and columns are in pixels. Blue: Exact Route, Red: Estimated Route. (100 pixels $=5 \mathrm{~mm})$. Figure is best viewed in color 
3.11 (a) Circular movement tracking error in $\mathrm{mm}$ with finger radius $7.5 \mathrm{~mm}$ and no noise (b) Angle Sweep movement with Finger radius $7.5 \mathrm{~mm}$

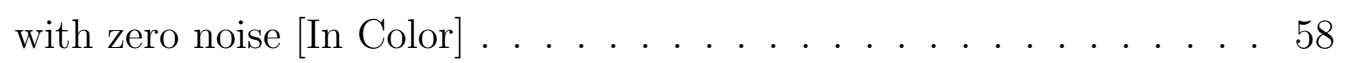

3.12 (a) Microchip projected capacitive touch board (top view) (b) MPLAB PCAP controller detecting track of two fingers, shown in red color. . 59

3.13 XPS NewPort motion stage controller. . . . . . . . . . . . . . 60

3.14 Robotic finger moving in a diagonal path. True path is shown in blue and estimated path is shown in green. Mean error is $2 \mathrm{~mm}$. Figure best viewed in color. . . . . . . . . . . . . . . . 61

3.15 Capacitive touch panel hardware testing using motion stage. . . . . . 61

3.16 Fourier log magnitude and phase for interleaved and angled cross. It can be seen from the magnitude plot that angled cross has high frequency values at multiples of 45 degrees whereas the interleaved pattern has high frequency values at multiples of 90 degrees (best viewed in color). . . . . . . . . . . . . . . . . .

3.17 (a) Hybrid pattern no. 1 consisting of Fourier Magnitude of Angled Cross and Phase of Interleaved Pattern, (b) Hybrid pattern no. 2 consisting of Fourier Magnitude of Interleaved and Phase of Angled Cross Pattern. ..................... . . 6 . . . . . . .

3.18 Interleaved sensor with unoptimized gap regions not included as part of sensing area (best viewed in color). . . . . . . . . . . . 65

3.19 Redesigned interleaved sensor with curved corners to increase the efficiency of the sensor (best viewed in color). . . . . . . . . . . 66

3.21 Simulation results for sensor patterns change in capacitance with 3D plots.

4.1 Simplified block diagram of a traditional capacitive touch panel system. 75 
4.2 Simple relaxation oscillator topology. Figure reproduced from [8]. . . 76

4.3 Simple sigma delta modulator topology. The Pseudo Random Sequence (PRS) generator is responsible for controlling the switch between external and sensor capacitor. Figure reproduced from [9] with simplification. . . . . . . . . . . . . . . . 77

4.4 Waveform showing complete operation for Charge Voltage Divider. In (a) without presence of finger, the charges settle in same amount of time which is not the case when there is finger touching the panel

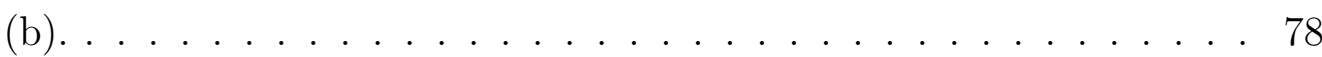

4.5 Graph showing voltage accumulated over time for charge transfer technique.

4.6 Block diagram explaining the measurement process of a capacitive touch controller. A square $N \times N$ panel is shown, although panels can be rectangular as well. . . . . . . . . . . . . . . . . . . 80

4.7 In this figure, (a)-(b) and (c)-(f) represent the two and four possible cases that need to be considered by CSAIM for $k=1$ and $k \geq 2$ respectively. ...................... . . 89

4.8 Fingers rested on a touch panel and the sparse signal arising from it. 102

4.9 Architectural diagrams for (a) BMP (b) BOMP/Block-CoSaMP showing all the steps involved for signal recovery. . . . . . . . . . . . 106

4.10 Architectural diagram of BMPNILS showing all steps involved in signal recovery. . . . . . . . . . . . . . . . . . . 107

4.11 RMSE versus $f$, with sparsity $k$ and $N$ kept constant at 10 and 600 respectively. . . . . . . . . . . . . . . . . . 111

4.12 Recovery of unknown vector using CSAIM, BCSBM, GMP and BMPNILS with Gaussian noise (figure best viewed in color). . . . . . . . . 112 
4.13 RMSE versus number of fingers $F$ for CSAIM, GMP, BCSBM and BMPNILS. $F$ is increased from 1 to 10 while keeping $N$ constant at 400. (figure best viewed in color) . . . . . . . . . . . . . . 113

4.14 RMSE versus number of fingers $F$ for BMPNILS, GMP, SCPMP, SCPHT and CoSaMP. $F$ is increased from 1 to 10 while keeping $N$ constant at 400 (figure best viewed in color) . . . . . . . . . . . . . 114

4.15 RMSE versus number of fingers $F$ for BMPNILS, BOMP, BMP, Block-CoSaMP. $F$ is increased from 1 to 30 (three people using the panel at the same time) while keeping $N$ constant at 600 (figure best viewed in color $)$. . . . . . . . . . . . . . . . . . . . 115

4.16 Comparison of number of FLOPS required between CSAIM, GMP, BCSBM and BMPNILS. In this figure, the number of fingers touching the panel is changed from 1 to 10 while keeping $N$ constant at 400 (using a $80 \times 80$ touch panel). Figure is best viewed in color. . . . 116

4.17 Comparison of number of FLOPS required between BMPNILS, GMP, SCPMP, SCPHT and CoSaMP. In this figure, the number of fingers touching the panel is changed from 1 to 10 while keeping $N$ constant at 400 (using a $80 \times 80$ touch panel). Figure is best viewed in color. . 117

4.18 Comparison of number of FLOPS required between BMPNILS, BOMP, BMP and Block-CoSaMP. In this figure, the number of fingers touching the panel is changed from 1 to 30 (three people using the panel at the same time) while keeping $N$ constant at 600 (We are using a $118 \times 118$ inch touch panel). Figure is best viewed in color. . . . . . . 118

4.19 Power consumption comparison for CSAIM, BCSBM, GMP and BMPNILS.

5.1 Wire grid sensor pattern with finger modeled as a 2-D circle . . . . . 126

5.2 A zoomed in image of finger response on the panel. Notice that a single finger covers many pixels. . . . . . . . . . . . . . . 127 
5.3 Figure depicting sensor readings and coarse peak position. . . . . . . 129

5.4 Figure depicting peak position derived by subpixel linear interpolation.129

5.5 An example diamond pattern column showing fine resolution values for each sensor $\mathrm{x} 0-\mathrm{x} 9 \ldots \ldots$. . . . . . . . . . . . . . . . . . . . . .

5.6 Varying pitch angle of the finger while the position of the touch panel contact remains unchanged. . . . . . . . . . . . . . . . . 131

5.7 Finger superimposed on a diamond grid. r1-r12 and c1-c9 depict the number of sensors triggered due to finger presence. . . . . . . . . 132

5.8 Finger of radius $=7.5 \mathrm{~mm}$ moving in a diagonal path across the interleaved grid touch panel (zoomed in, without noise). The units for rows and columns are in pixels (100 pixels $=5 \mathrm{~mm})$. Figure is best viewed in color. . . . . . . . . . . . . . . . . . . . 134

5.9 Finger of radius $=7.5 \mathrm{~mm}$ moving in a circular path across the interleaved grid touch panel (without noise). The units for rows and columns are in pixels $(100$ pixels $=5 \mathrm{~mm})$. Figure is best viewed in color. . . . . . . . . . . . . . . . . . . . . 138

5.10 Angle sweep performed by a $7.5 \mathrm{~mm}$ radius finger for different interpolators. The units for rows and columns are in pixels (100 pixels $=$ $5 \mathrm{~mm})$. Figure is best viewed in color.

5.11 Bias in estimator result due to the same column value input to the estimators throughout the motion of the finger in the interleaved pattern. Notice that the horizontal axis is scaled to show the bias. Units for rows and columns are in pixels. (100 pixels $=5 \mathrm{~mm})$. Figure is best viewed in color. . . . . . . . . . . . . . . . . . . . . . . 140

5.12 Bias in subpixel estimators. The column value was changed from 450 to 750 in steps of 10 after a single vertical motion by the finger and then mean of the estimator result was subtracted from the true value to get the bias. Notice that COM estimator has poor performance when compared with other estimators. 
5.13 Minimizing the edge effect on interleaved grid. (a) Original result. Notice that due to the finger diameter being 300 pixels, the error due to edge effect starts to show after 900 pixels on x-axis when the finger starts leaving the screen (b) After correction. The error starts appearing now from 1000 pixels onwards (figure is best viewed in color).142

5.14 Subpixel error $E$ versus SNR for a steady single finger placed on a touch panel. Figure is best viewed in color. . . . . . . . . . . . . . 145

5.15 Computation time ( $\mu$ sec) vs SNR for a steady single finger placed on a touch panel. Figure is best viewed in color. . . . . . . . . . . . 146

5.16 Framework depicting the recovery of noisy capacitive touch signals using BMPNILS and subpixel peak estimation using Gaussian interpolator. . . . . . . . . . . . . . . . . . . . . . 148

6.1 Recovery of noisy capacitive touch panel image using dictionaries trained by K-SVD and Block K-SVD and comparison of their PSNR with image recovered using a Discrete Cosine Transform (DCT) dictionary. . . . . . . . . . . . . . . . . . . . 154 


\section{List of Tables}

1.1 Resistivity of different materials in $\mathrm{Ohm}-\mathrm{cm}$. . . . . . . . . . . . 15

3.1 Diagonal movement tracking error comparison. Smallest error value is shown in bold. . . . . . . . . . . . . . . . . . 52

3.2 Circular movement mean tracking error $(\mathrm{mm})$ with finger radius $7.5 \mathrm{~mm}$ and no noise. . . . . . . . . . . . . . . . . . 56 56

3.3 Angle sweep mean tracking error ( $\mathrm{mm}$ ) with finger radius $7.5 \mathrm{~mm}$ and no noise. . . . . . . . . . . . . . . . . 5 56

3.4 Performance comparison of hybrid patterns with angled cross and interleaved pattern. . . . . . . . . . . . . 63

4.1 Proposed algorithms comparison . . . . . . . . . . . . . . . . . . . . . 122

4.2 Accumulated Stack Size per Algorithm in kilo Bytes . . . . . . . . . . 123

5.1 Three-point subpixel interpolation algorithms. Formulas taken from $[10] . \ldots \ldots \ldots \ldots$

5.2 Interleaved pattern diagonal movement tracking error comparison for varying pitch angle. Smallest error value is shown in bold. . . . . . . 135

5.3 Interleaved pattern circular movement mean tracking error ( $\mathrm{mm}$ ) with

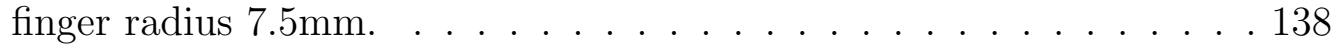

5.4 Interleaved pattern angle sweep mean tracking error $(\mathrm{mm})$ with finger radius $7.5 \mathrm{~mm}$. . . . . . . . . . . . . . . . . . . . 140 


\section{Abbreviations}

$\begin{array}{ll}\text { AMR } & \text { Analog Multi-touch Resistive } \\ \text { BCSBM } & \text { Block Compressive Sensing using Binary Matrices } \\ \text { BMPNILS } & \text { Block Matching Pursuit with Non-Iterative Least Squares } \\ \text { CM } & \text { Contract Manufacturer } \\ \text { COM } & \text { Center of Mass } \\ \text { CSAIM } & \text { Compressive Sensing for Augmented Identity Matrices } \\ \text { CoSaMP } & \text { Compressed Sampling Matching Pursuit } \\ \text { FEM } & \text { Finite Element Method } \\ \text { FLOPS } & \text { FLoating-point Operations Per Second } \\ \text { GIS } & \text { Geographic Information System } \\ \text { I2C } & \text { Inter-Integrated Circuit } \\ \text { ITO } & \text { Indium Tin Oxide } \\ \text { MOM } & \text { Method Of Moments } \\ \text { MP } & \text { Matching Pursuit } \\ \text { MVVM } & \text { Model View View Model } \\ \text { ODM } & \text { Original Design Manufacturer } \\ \text { OEM } & \text { Original Equipment Manufacturer } \\ \text { OMP } & \text { Orthogonal Matching Pursuit } \\ \text { POS } & \text { Point Of Sales } \\ \text { SAW } & \text { Surface Acoustic Wave } \\ \text { SV } & \text { Spatial Visualization } \\ \end{array}$





\section{Chapter 1}

\section{Introduction}

This is the age of touch technology. Once considered revolutionary, it is now ubiquitous. A touch screen is an electronic display that the user can control through single or multi-touch gestures by touching the screen with one or more fingers, thus enabling them to interact directly with the display rather than using a traditional keyboard-mouse interface. Apart from bare fingers, a touch screen can take input commands through a stylus or special gloves. A simple use case scenario would be zooming in on a picture using two fingers or panning a map using one finger.

Touch screens are commonly found in smartphones, tablet computers, gaming consoles and kiosks, either as an integrated layer below the cover glass, or as an overlay that can be attached to any display making it touch enabled.

In devices such as Automated Teller Machines (ATM) and information kiosks, touch technology has brought a revolution as the user interface for touch is simpler and easier to understand than that of a keyboard-mouse system. Also, in the case of information kiosks, the traditional way of user input through a keyboard does not allow an intuitive, rapid or precise interaction with the display.

Historically, the touch sensor and controller firmware were not sold by display panel manufacturers. They was available in market through a variety of after-market system integrators. In recent years, the trend has changed due to wide demand of 
touch interface and $\mathrm{TV} /$ notebook display manufacturers have begun to incorporate touch sensors in the fundamental design of their products.

Broadly speaking, touch technology can be divided into two categories based on their application/deployment:

1. Smartphones, tablets and laptops

2. Retail outlets and wall displays

The technology behind each of the above is the same, but the differentiating factor is the size of the display. There is no standard definition for what constitutes a large-scale retail outlet display, but in this thesis, we would consider a touch screen greater than 30 inches in the second category. From this point onward, we will refer to large touch screens used in retail outlets as touch panels and the rest as simply touch screens.

Touch market technology for smartphones, tablets and laptops is established and saturated as millions of touch screen phones are sold every year around the world. Mobile applications have adapted to touch input in their user interface (UI) and although multiple technologies are being used, capacitive touch technology is the leader in the smartphone industry. The same can be said for tablets and laptops. Capacitive touch screen with its non moving parts and high precision took over resistive touch technology soon after the release of iPhone in 2007.

Currently, the touch panel industry has no monopoly. There are multiple technologies that are currently used for touch panels with resistive touch being the most dominant one due to its low cost. Although, infrared touch is also capturing the market but capacitive touch is fast growing to overcome both and lead the market just like it did in the smartphone industry [3].

There are different places in retail outlets where touch panels are used. Some of them are listed below: 
1. Point of sales (POS) systems

2. Kiosks

3. Vending machines

4. Digital signage

5. System control and office automation

POS has the highest application for touch panels at the moment but after the advent of Internet of Things (IoT), rapid growth of applications in digital signage and automation is expected.

The touch panel industry is a combination of vendors and manufacturers and the supply chain is complex as depicted by Figure 1.1.

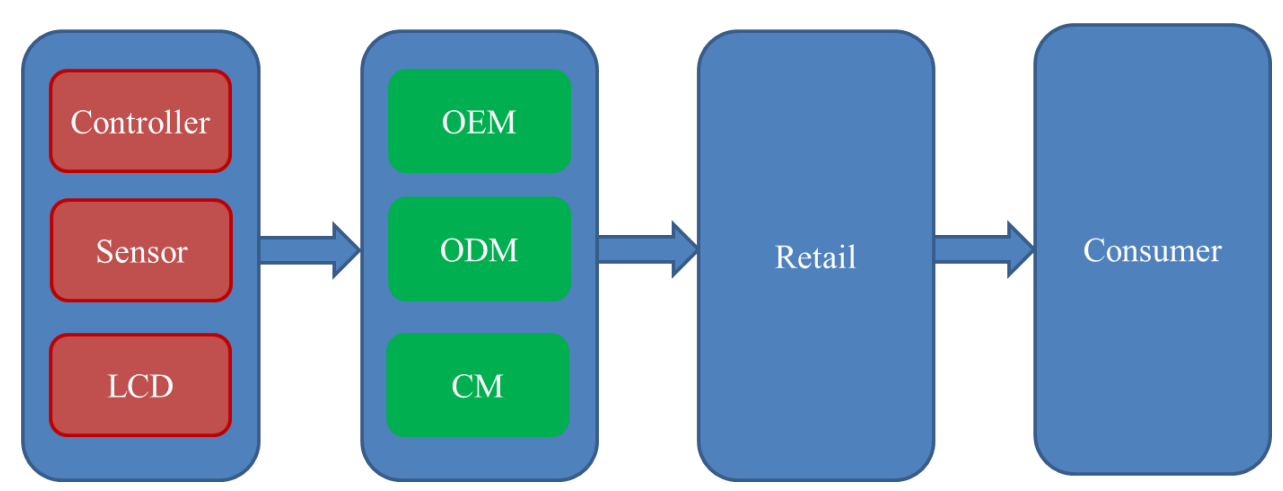

Figure 1.1: Touch panel supply chain comprises of Original Equipment Manufacturer (OEM), Original Design Manufacturer (ODM) and Contract Manufacturers (CMs) supplying various components of the touch panel to the retail market.

\subsection{Touch Panel Market Share and Forecast}

According to market research [1], the global touch panel market shipped around 1.02 million units in 2014 and is expected to ship more than 3.1 million units in 2019 , with an annual growth rate of $25 \%$ as shown in Figure 1.2. 


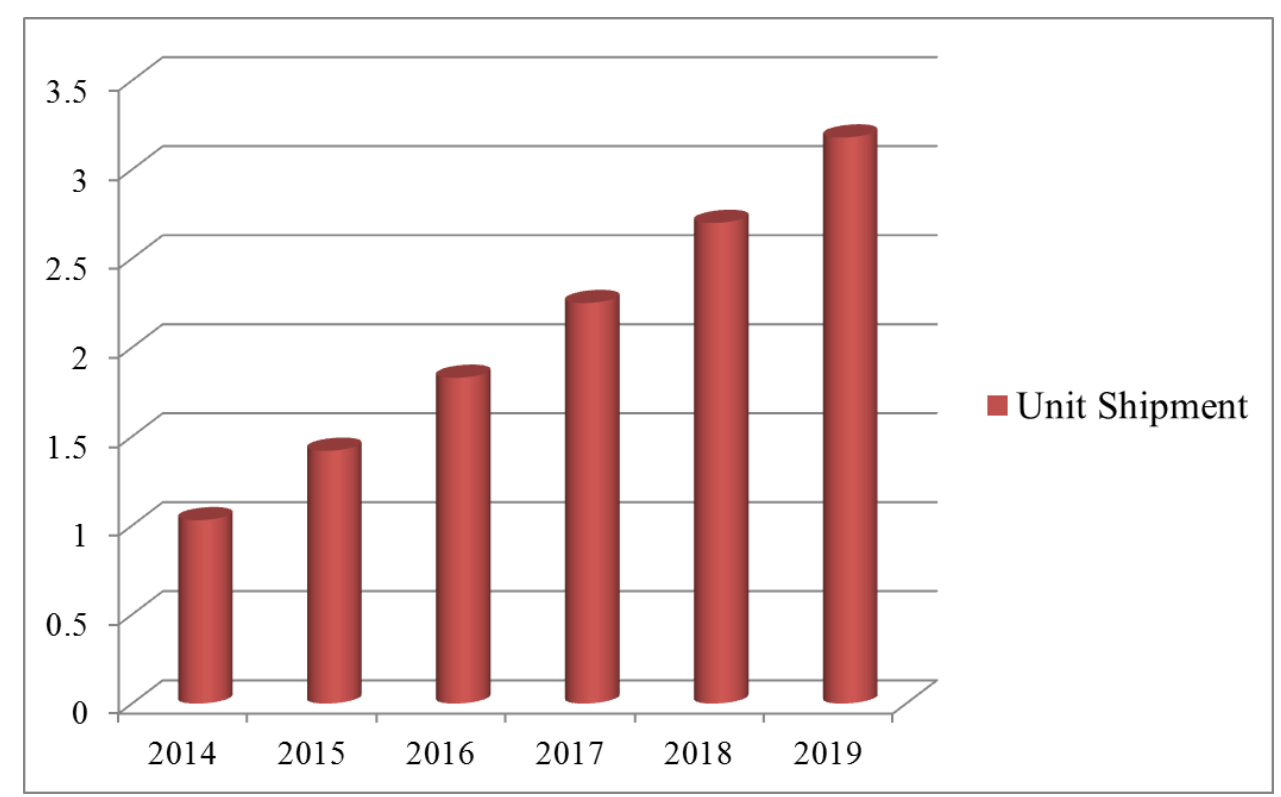

Figure 1.2: Global retail market touch panel shipment forecast 2014-2019 (million units) [1].

There are a number of touch screen technologies that use different methods for sensing touch as shown in Figure 1.3. We will explain the basics of these technologies in this chapter but our main focus will be on projected capacitive touch technology. In order to differentiate between projected capacitance and surface capacitance, from this point on we will refer to projected capacitive touch technology as capacitive touch technology and surface capacitive as is, unless stated otherwise. Figure 1.4 and 1.5 give the 2014 and projected 2019 market share of various touch technologies in retail market $[1]$.

As seen in Figure 1.4 and 1.5, resistive touch technology was dominating retail outlets in 2014 at $47 \%$ and will continue to do so in 2019 at $42 \%$. However, capacitive touch and infrared share is rising and they will overcome resistive touch in years to come. Capacitive touch technology has not dominated the market over the five years compared, largely because of the high cost. As mobile shopping and self service kiosks with increasing complex UI become more mainstream, further need will arise for high precision multi-touch kiosks. Therefore, market share for capacitive touch will keep on rising as forecast in the pie-charts shown in Figure 1.4 and 1.5. Industry has started doing research in manufacturing very large-scale capacitive 


\begin{tabular}{|c|c|c|c|c|}
\hline & Touch Technology & 2"-17" & $10 "-30 "$ & $>30^{\prime \prime}$ \\
\hline 1 & Projected Capacitive & M & M & $\mathbf{E}$ \\
\hline 2 & Surface Capacitive & & M & \\
\hline 3 & Resistive & $\mathbf{L}$ & $\mathbf{L}$ & M \\
\hline 4 & Surface Acoustic Wave & & M & $\mathbf{L}$ \\
\hline 5 & Infrared & & M & M \\
\hline 6 & Camera Based Optical & & M & M \\
\hline 7 & Vision Based & & & $\mathbf{E}$ \\
\hline \multirow[t]{5}{*}{8} & Force Sensing & & $\mathbf{E}$ & \\
\hline & & & & \\
\hline & & M & Mainstream & \\
\hline & Key: & $\mathrm{L}$ & Low-Volume & \\
\hline & & E & Emerging & \\
\hline
\end{tabular}

Figure 1.3: Touch technologies classification [2].

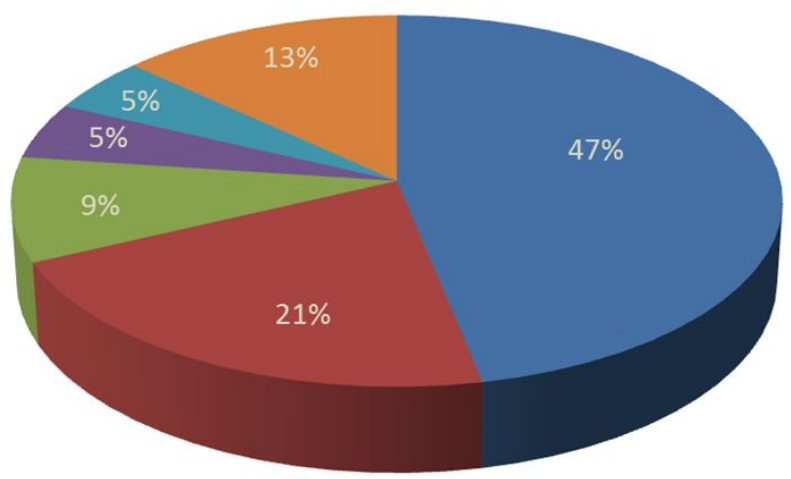

\author{
nesistive \\ - Projected Capacitive \\ n Infrared \\ - Surface Capacitive \\ - Surface Acoustic Wave \\ - Others
}

Figure 1.4: Touch panel market share in global retail market by technology in 2014 [1] (figure best viewed in color).

touch panels. Microsoft [11] and Zytronic [12] have recently started producing up to 82 inch capacitive touch panels.

Below, the key technologies are discussed in detail. We will go through their technical principles as well as where they stand in terms of forecasts and what the future holds for them. 


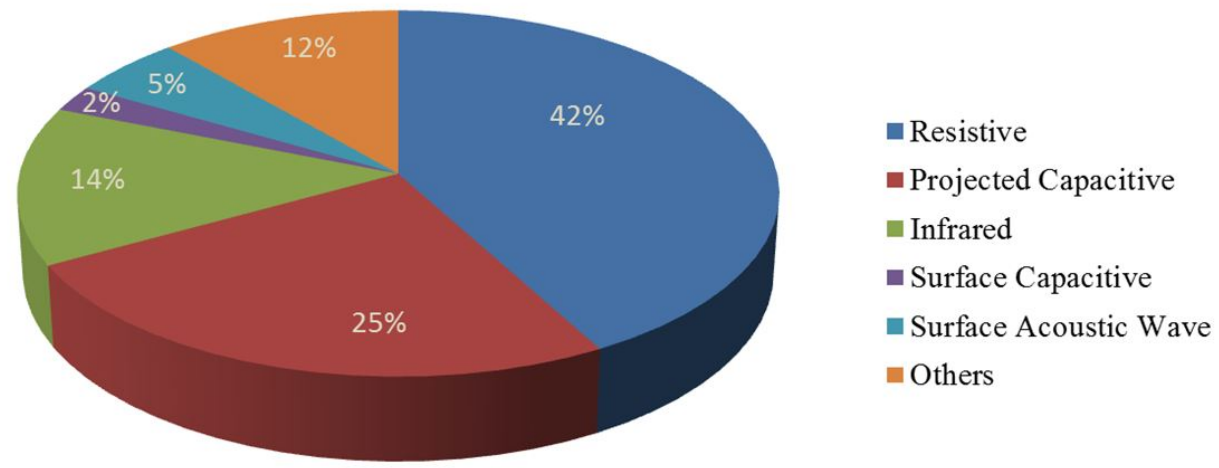

Figure 1.5: Forecast - Touch panel market share in global retail market by technology in 2019. [1] (figure best viewed in color).

\subsection{Touch Technologies Overview}

\subsubsection{Resistive-based Technology}

Resistive touch is the oldest touch technology (approximately 40 years old) currently in production, although its share has reduced considerably over the years. In 1977, the first transparent resistive touch panel was developed by Elo TouchSystems [7].

For a typical resistive touch screen, two layers of conductive coating are separated by small insulating spacer dots and a voltage is applied to one or both sheets depending upon the application (Figure 1.6).

This is the amended thesis, the sheets make contact and the resistance of the spacer dots creates a voltage divider circuit at the contact point which is then picked up by the touch controller as a valid touch input. Because the only requirement for generating a touch signal is applied pressure, resistive touch screens can work with gloves on and with nearly any stylus shaped object. This useful feature increases the reliability and usability of the technology, however as pressure is required to press the conductive sheets together, devices incorporating resistive touch are more prone to damage. 


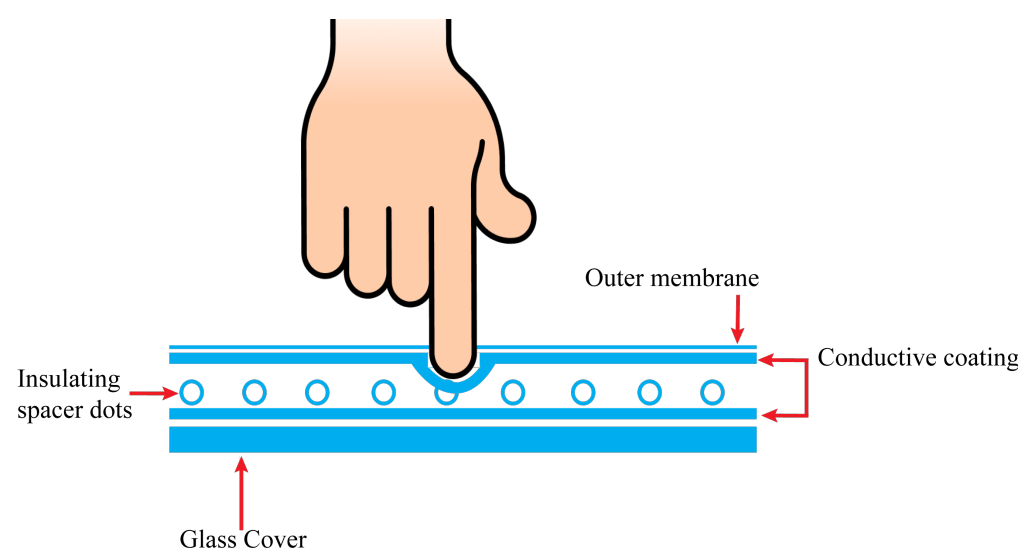

Figure 1.6: In a basic resistive touch screen, the pressure of the finger is used to create electrical contact between the two conductive layers causing a voltage divider circuit to be formed. Source: [3].

\subsubsection{Large-scale Multi-touch Resistive Touch Panels}

Touch resistive technology was used only in single touch detection devices for many years and it was only around 2009 after the invention of iPhone, when two small firms namely Stantum [13] and now defunct Touchco [14] started researching and producing small prototypes of multi-touch resistive touch screens. Stantum touch screen contains two Indium Tin Oxide (ITO) layers which are separated by an insulating medium. This multi-touch resistive sensing hardware provides two dimensional data from which the data processing algorithm removes the background noise and identifies the valid touch points [7]. The technology was labeled Analog Multi-touch Resistive (AMR). Figure 1.7 shows the working of a large-scale AMR panel.

In Figure 1.7, each box is around 10-20 $\mathrm{mm}$ wide and that can cause an issue when two fingers are held close together on the panel, as it may report only a single finger touch. On the other hand, if the box is made smaller than $10 \mathrm{~mm}$, it increases the manufacturing cost of the panel by a considerable amount. Apart from these fundamental issues, AMR technology suffers from less durability and low optical performance because a touch has to be registered using physical force [15]. As pointed out in [7], AMR will not have any significant share on the consumer electronics market in the years to come. 


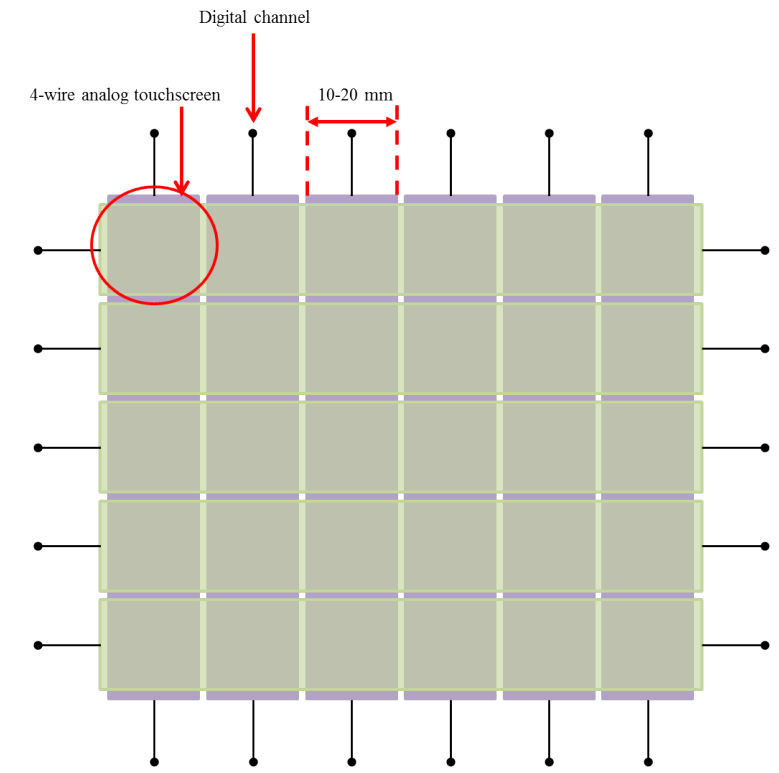

Figure 1.7: In an AMR touch sensor, the conductive layers are shaped as strips such that each intersection of strips forms a square, Each square is an independent 4-wire analog touch sensor. Source: [3].

\subsubsection{Surface Acoustic Wave-based technology}

Surface acoustic wave (SAW) was first designed and manufactured by Zenith and Elo TouchSystems [16] in 1985. In the late 1980s, there was a production boom for SAW devices when capacitive touch systems were not in large-scale production. The technology employs acoustic wave transducers, both receivers and transmitters, which are installed at the corners of the panel. The transmitter sends out bursts of ultrasonic Rayleigh waves that are reflected towards the receiver by an array of reflectors along the sides of the panel. When a finger or stylus approaches, a portion of the transmitted wave is absorbed and thus there is a delay in the time taken for the wave to reach the receiver $[15,7]$. This delay is picked up by the touch controller as a valid touch input. There is room to measure the touch pressure by measuring the amount of wave absorption and thus computing the $\mathrm{Z}$ axis value but it has never been widely practiced in current devices.

Surface acoustic wave devices have high light transparency and can be used for multi-touch detection. They are generally high resolution and can work even when 


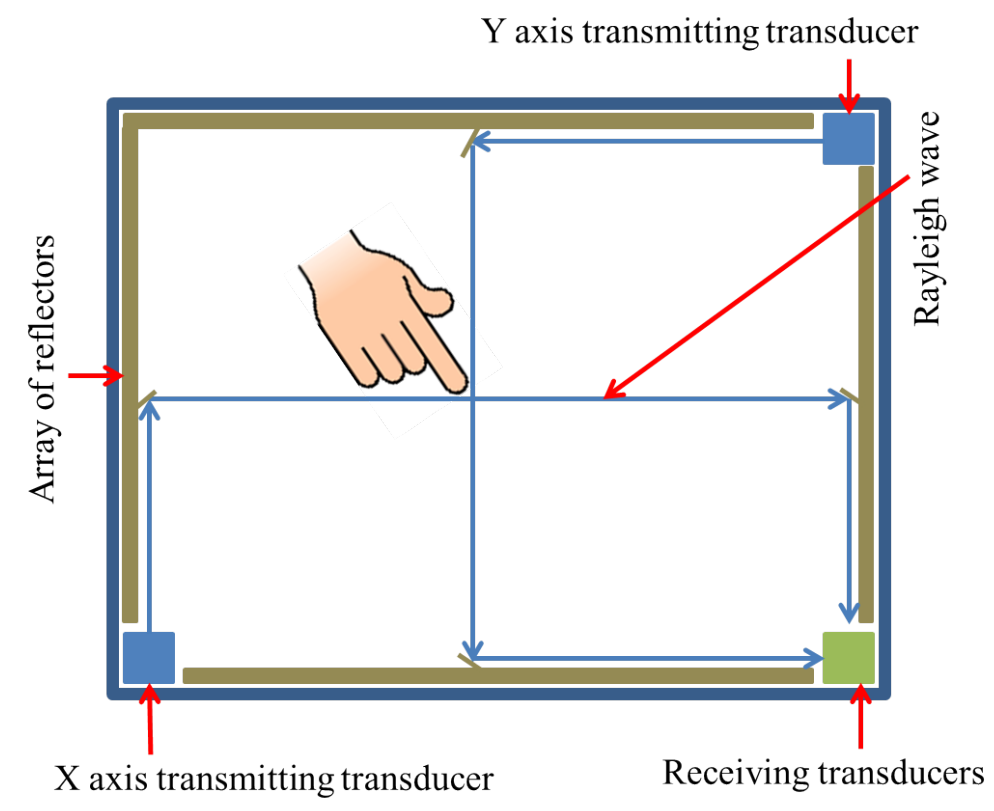

Figure 1.8: Multi-touch system based on SAW technology. In the figure, touch causes the wave to be absorbed causing a delay on its way to the receiving transducers. This disturbance can be picked up by the controller as a possible touch point. Source: [3].

the display glass is damaged. However they are highly sensitive to environmental conditions such as temperature and humidity and are costly to manufacture. Another issue that hinders a widespread use of SAW devices is the considerable amount of pressure that is required for registering touch which limits the usability of the technology.

\subsubsection{Large-Scale SAW Touch Panels}

Due to the aforementioned issues, SAW never became popular as a preferable technology for large-scale touch panels over the years. It was used extensively in medium sized kiosks such as ATM. However, in May of 2016, Elo unveiled its new eSAW technology calling it the next generation of SAW. The Elo Intellitouch eSAW panel is available up to 55 inches in size and has the ability to measure touch response on $\mathrm{Z}$ axis as well [17]. At the time of this writing, the eSAW technology is very new and untested, and thus it can not be said how well it will perform in the coming years. 
According to market research, 0.05 million units of SAW were shipped in 2014 and this figure will rise to 0.17 million in 2019 [1].

\subsubsection{Infrared-based technology}

The first ever commercial application of infrared technology was unveiled in 1983 as HP-150, HP's first touch microcomputer. Infrared touch technology is very unique because of its extremely low manufacturing cost and high portability. The early infrared touch systems detected single touch and were composed of a frame containing infrared LEDs and photo-detectors on the sides. Each LED was pulsed in sequence and the light emitted was received by the photo-detectors. As shown in the Figure 1.9, this mechanism forms a grid of infrared light which gets disturbed when a foreign object touches the panel. The disturbance is read by the touch controller determining the location of the touch. Due to this simple design, infrared screens can detect any foreign object touching the panel, similar to the resistive touch panels. The resolution of the touch panel can be increased by adding more transmitters. There is an upper limit on the accuracy of infrared panels as the maximum resolution of the panel is proportional to the minimum size of the transmitters installed in the frame.

\subsubsection{Large-Scale Infrared Touch Panels}

Infrared is a widely manufactured and used technology for large-scale touch panels. The reason being that they are so cheap to manufacture, many small OEMs have started producing infrared panels in large quantities, which is further bringing the cost down. The multi-touch version of infrared touch panel works on the principle of imaging. The difference is in the detection algorithm of the touch controller. Instead of one to one correspondence between detector and transmitter, multiple photodetectors record their level of light intensity and produce a 'shadow image' of the touch point [18]. This process is repeated over a period of time, creating a sequence 


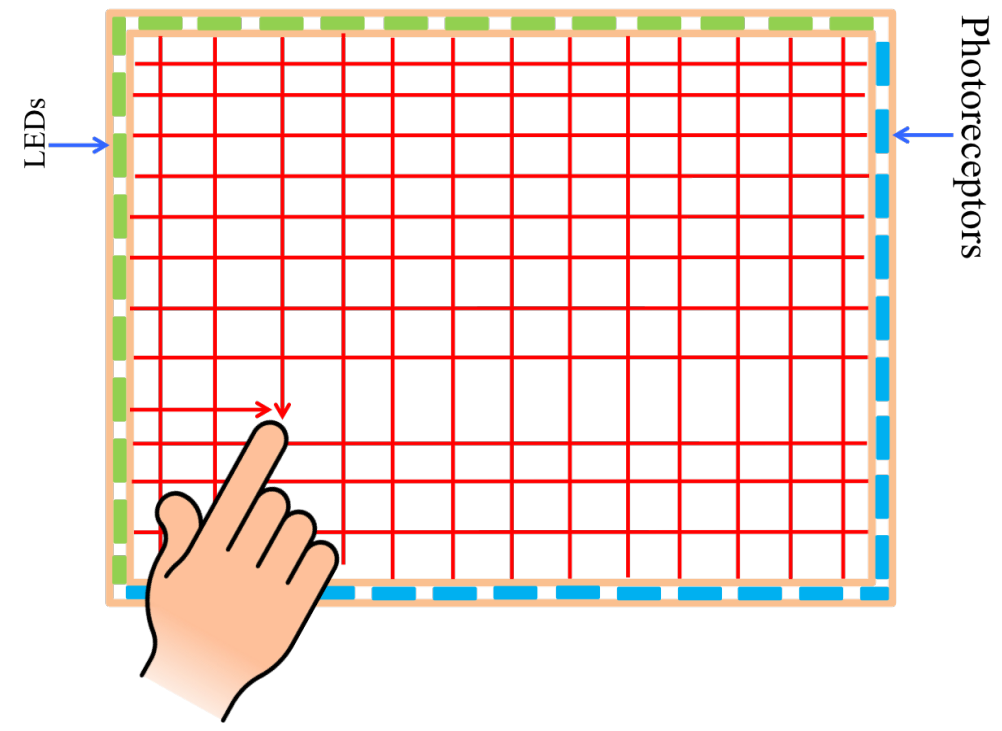

Figure 1.9: Phenomenon of infrared sensing. Blue and green rectangles represent the photo-receptors and the LEDs respectively. Touching the screen causes a break in the light path as shown by red arrows. Figure reproduced with changes from [2] and is best viewed in color.

of images which can be used for tracking multiple objects. The major providers of this technology are PQ Labs [19], ZaagTech [20] and Citron [21]. According to market research, the market shipped 0.09 million units in 2014 and is expected to ship 0.46 million units in 2019 [1].

\subsubsection{Surface Capacitive-based technology}

In surface capacitive technology, the panel is usually covered with a conductive coating constructed from Indium Tin Oxide (ITO) which represents lower plate of a capacitor. The human finger behaves as the upper plate with cover glass acting as the dielectric. The touch controller can easily determine the location of touch by measuring the voltage at four corners of the panel. This technology has no moving parts so it is durable but suffers from limited resolution. It does not support multitouch and thus can only be used in simple applications such as ATM kiosks and industrial control panels. Figure 1.10 explains the working of a surface capacitive panel. 


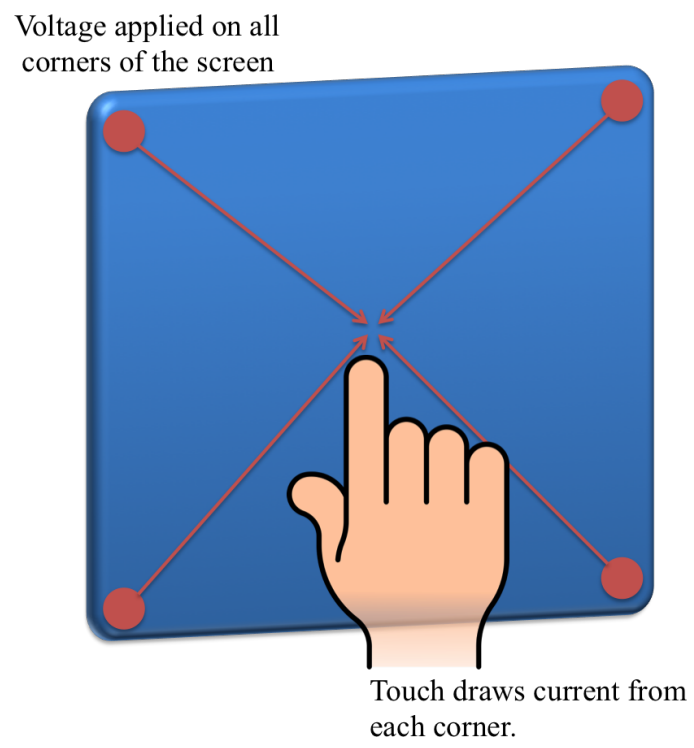

Figure 1.10: Working of a surface capacitance based touch panel.

\subsubsection{Large-Scale Surface Capacitance Touch Panels}

Surface capacitance is a highly accurate and durable technology which can be scaled up without any significant cost increase. However, due to lack of multi-touch capability, its use is declining slowly (Figure 1.5). The biggest use of surface capacitance can be found in amusement machines and legacy gaming.

\subsubsection{Projected Capacitive-based technology}

\subsubsection{History}

Projected capacitive touch technology is not new and has been around for more than 50 years. It is generally agreed among historians that the first finger capacitive driven touch screen was invented by E. A. Johnson in 1965 while working at Royal Radar Establishment in United Kingdom [4]. His work was published in electronics letters under the title "Touch display - a novel input/output device for computers". Johnson explained his work further in "Touch Displays: A Programmed ManMachine Interface", published in Ergonomics in 1967 [22]. His invention is shown in Figure 1.11. 


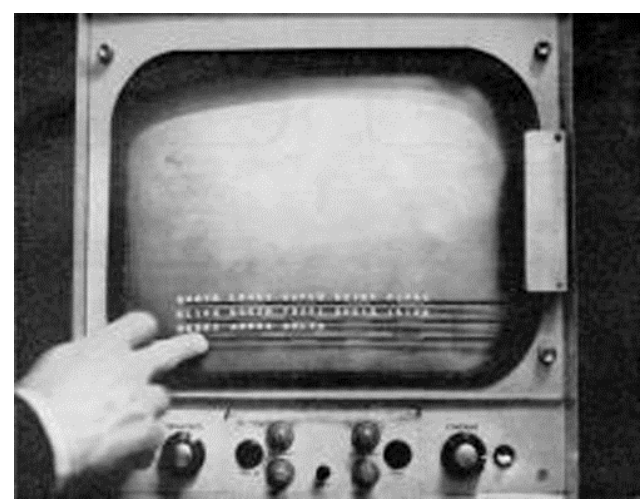

Figure 1.11: E. A. Johnson with his invention in 1967 [4].

During 1985, surface capacitance based technology was commercialized by Microtouch systems and in the 1990s, many US based companies started producing capacitive touch panels and accompanying touch controllers. The sensors were manufactured using Indium Tin Oxide (ITO), which is still widely used in manufacturing low cost capacitive touch screens. Dynapro and MicroTouch systems (later acquired by $3 \mathrm{M}$ touch systems) were one of the first companies to introduce surface capacitance systems in the market. 3M Touch system's first touch screen product named as Near Field Imaging was based on technologies developed by Microtouch and Dynapro and was launched in 2001.

In 1994, R. P. Binstead designed a projective capacitance circuit using a pattern consisting of microfine wires. A single microfine wire thickness is around $25 \mathrm{mi}$ cron. The technology was licensed to Zytronic and Visual Planet in 1998 and 2003 respectively.

The release of Apple iPhone in 2007 brought a revolution to the smart phone industry. It was the first touch screen smart phone which employed capacitive multi-touch capability to a limited degree [23]. Also in the same year Microsoft released Surface 1.0 [24], which was basically an interactive table surface capable of sensing more than five fingers at the same time. This was used as a tool in collaborative and informative learning environment. Surface 1.0 was followed by Surface 2.0 in 2011. Microsoft re-branded the technology as PixelSense in 2012-2013.

As capacitive touch technology makes its way into our daily lives now more than 
ever, it is amazing how simple this technology actually is. The working principle of capacitive touch technology is explained below.

\subsubsection{Working Principle of Capacitive Touch Technology}

A capacitive touch screen panel works on the principle of capacitance/charge measurement. The panel is coated with a material capable of storing electrical charge. When a conductor such as a human finger is brought in contact with the panel, a small amount of charge is drawn to the point of contact. The circuit located at the border (or edges) of the panel measures the charge difference and transmits this information to the touch controller for processing. As conductivity of charge plays a central part in workings of capacitive touch screens, they cannot be operated using regular gloves or plastic stylus. Due to the transparent nature of the conducting material, capacitive touch panels have excellent clarity and are unaffected by common contaminants like dirt or grease.

The manufacturing material used for capacitive electrodes can be Indium Tin Oxide (ITO), Copper or Silver. The performance of the panel depends upon the type of material used for construction of electrodes. The increase in resistivity decreases the sensitivity of the whole system, thus decreasing the performance.

We know that the ability of an electrode to transfer charge is inversely proportional to the resistivity of the conductor. Some commonly used materials and their resistivity values are given in Table 1.1 [25].

The recommended practice is to use low resistance materials to increase the sensitivity of the sensor. An exception to this practice is the wide spread use of ITO which provides lower sensitivity than other materials. This drawback of ITO must be compensated for in the capacitance measurement algorithm by adjustment of scanning rate. However ITO is still used widely by touch screen manufacturers as it allows the capacitive sensor to be up to 90 percent transparent for both single and double layered solutions. 
Table 1.1: Resistivity of different materials in Ohm-cm

\begin{tabular}{|c|c|}
\hline Material & Resistivity $\rho($ Ohm-cm) \\
\hline Copper & $1.68 \times 10^{-6}$ \\
\hline Silver & $1.59 \times 10^{-6}$ \\
\hline Tin & $1.09 \times 10^{-5}$ \\
\hline Indium Tin Oxide & $1.05 \times 10^{-3}$ \\
\hline
\end{tabular}

In Projected Capacitive Touch (PCT), electrodes are etched on an insulator thus allowing accurate and flexible operation. There can be a single or double layer of conductive material and the choice is based on the type of application. These electrodes form an X-Y grid pattern and an external conductive object can change the voltage flowing through the electrode lines by forming a capacitor with the conductive traces. This phenomenon is illustrated in Figure 1.12.

The alternating current flowing is continuous across the conducting material surface, and when human body touches the screen, it starts conducting and hence the voltage drop at the touch point is captured as a touch event. Electronic circuits located under the ITO layer measure the distortion produced in voltage and transmit information to the main controller to translate the event into a meaningful gesture. The cost of manufacturing capacitive touch sensing devices (although decreasing with the increase in production volume) is greater than optical as well as resistive devices. Because the device must be able to sense changes in capacitance as small as few Femtofarads $\left(10^{-15} \mathrm{~F}\right)$, therefore the sensors are very sensitive to electromagnetic interference. The aim of a capacitive touch panel designer is to find the optimal point 


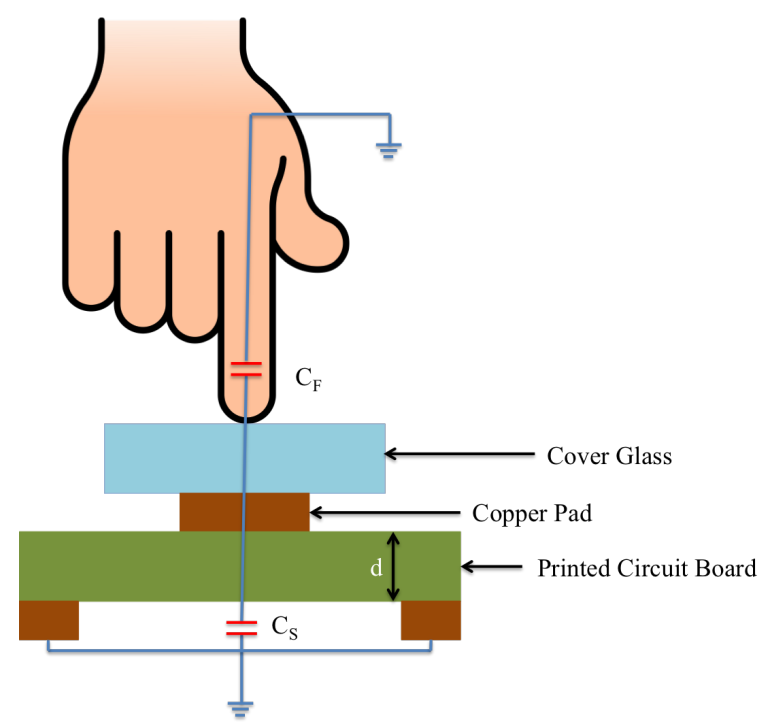

Figure 1.12: Phenomenon of capacitive sensing. Blue line represents a capacitive circuit formed between finger and copper pad. $\mathrm{C}_{\mathrm{F}}$ and $\mathrm{C}_{\mathrm{S}}$ represent the finger and sensor capacitance respectively whereas d represents the distance between the plates. Figure reproduced with changes from [5].

between sensitivity of the sensor and its immunity to noise [26]. Also capacitive touch sensors have the advantage that they can be integrated in a small area and thus are suitable for mobile phones and Personal Digital Assistant (PDA) devices. Projected capacitance can be further divided into self and mutual capacitance. Self (or absolute) capacitive sensing is used when the processor wants to determine only one finger touch location over the grid. Although self capacitance can measure multiple touches but it is not a common practice due to the phenomenon of ghost points (explained below). As a low cost solution it is still widely used in ATM kiosks and in information and education targeted kiosks. Smart phones like iPhone use a different type of touch sensing called mutual capacitance in which the processor can detect multi-touch.

Although both self and mutual capacitance are based on projected capacitive touch technology and in both techniques, capacitance variation is measured in column and row basis, they have notable differences as indicated by Figure 1.13.

In self capacitance, when a conductive object approaches the sensor, node to ground capacitance of a channel under the object increases. The controller scans all rows and 


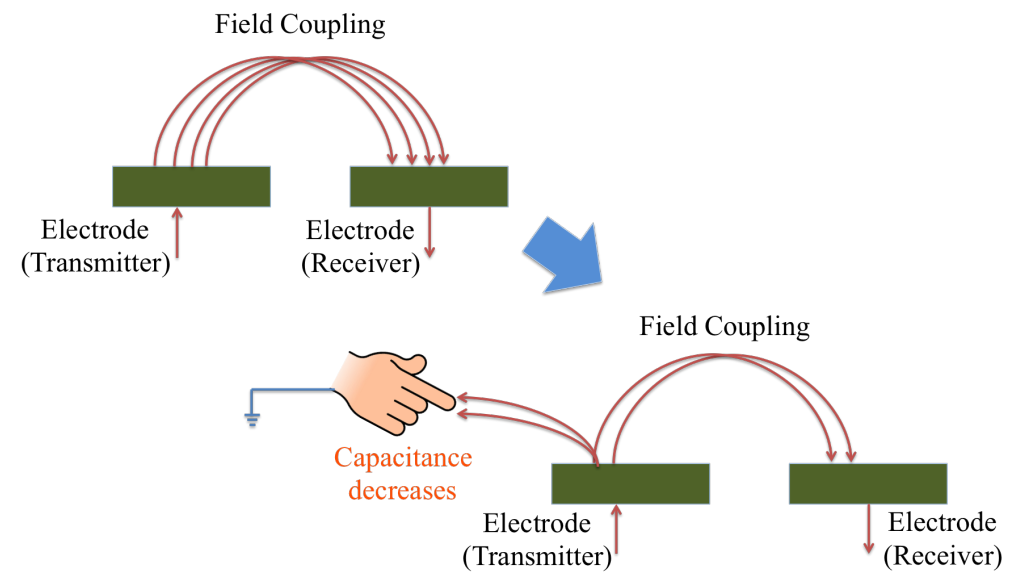

(a)

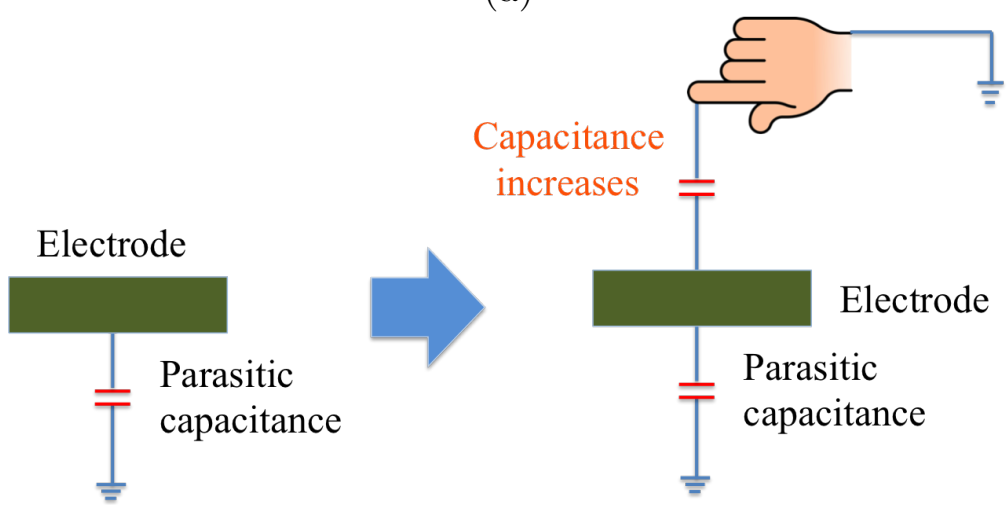

(b)

Figure 1.13: Figure depicting difference between self and mutual capacitance. (a) Mutual capacitance (b) Self capacitance.

columns to find out the exact location of a single touch. Note that, if the panel has 10 rows and 7 columns, scanning 17 channels can identify 70 touch points in theory. While this approach can be implemented for one touch or simple two touch gestures, it has limitations for more than two-touch (or complex) gestures. The system may detect two $x$ coordinates $(x 1, x 2)$ and two $y$ coordinates $(y 1, y 2)$ but it has no way of knowing that which $x$ coordinate goes with which $y$ and vice versa, therefore introducing two ghost points on the panel. In Figure 1.14, a self capacitive system will not be able to tell the difference between two finger touches, $[(x 1, y 2),(x 2, y 1)]$ and $[(x 1, y 1),(x 2, y 2)]$. However, self capacitance is still very popular because its ease of implementation in applications which require single touch gestures.

Mutual capacitance eliminates the ghost point problem encountered in self capac- 


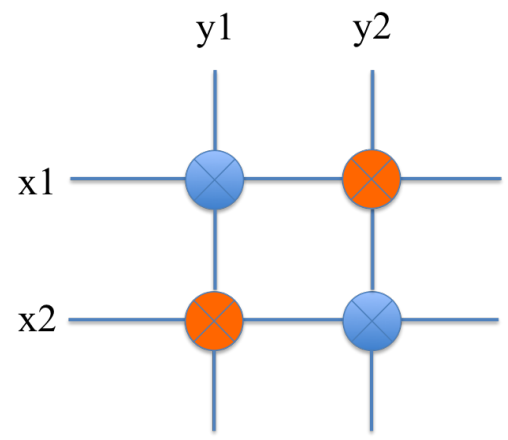

Figure 1.14: Self capacitance ghost point problem. A self capacitance system will not be able to differentiate between two finger touch $[(x 1, y 2),(x 2, y 1)]$ and $[(x 1, y 1),(x 2, y 2)]$.

itance systems. Usually mutual capacitance has two cross patterned layers with a dielectric in between. This two-layer design is the same as self capacitance but capacitance is formed at intersection of both layers.

During the scanning process, the upper layer drives the signal (usually a square wave) and the charge magnitude is sensed at each line of lower layer. The touch controller has to scan every single intersection of the driving lines with sensing lines. Thus for 70 nodes, 70 intersections have to be scanned, taking longer time than what is required for scanning the same number of nodes for self capacitance. Also signal sensitivity is lower because charge is measured between the intersections of electrodes rather than on the electrodes itself.

A typical capacitive touch panel has three major parts: physical sensor/analog front end, signal sampling and signal processing as shown in Figure 1.15. The physical sensor is responsible for changing its state when a conductive object is placed near the panel which is then sampled by the touch controller. The signal processing part is responsible for noise filtering and interpolation in order to recover the touch location.

The advantages of using capacitive touch technology are numerous. Some of the major advantages are listed below:

1. Capacitive touch panels support multi-touch thus enabling developers to create high-tech applications. 


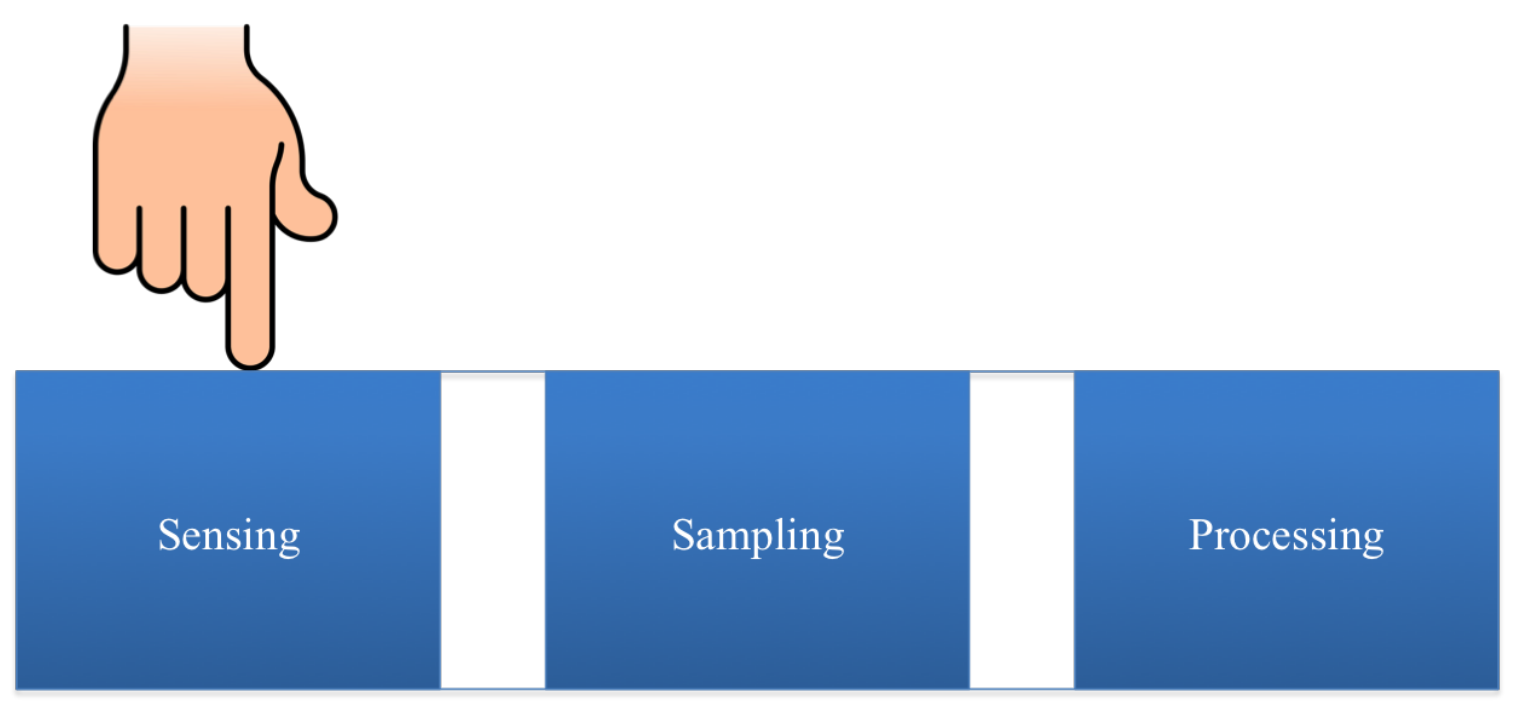

Figure 1.15: Three main components of a capacitive touch system

2. They do not require a bezel to operate and the resulting panel is a true flat surface that is aesthetically pleasing.

3. They are scratch-resistant and have a high endurance limit. They also do not require finger pressure to operate unlike resistive or SAW touch devices.

Some of the early critics of capacitive touch panels would point out the limitation that it cannot be used while wearing insulating gloves or with plastic stylus, but with advances in technology, capacitive touch supporting gloves and stylus are now available in market which allow the use of technology in harsh cold environments as well.

\subsection{Motivation}

When reviewing the academic literature, it can be seen that there is very little published research in the field of capacitive touch technology. Our work deals with sensor pattern design and testing, signal sampling and subpixel interpolation for accurate finger detection. Most of the research in these three fields has been done by industry and not by academia, therefore it is confidential and unpublished. 
The little available academic literature is concerned with designing more accurate, robust and noise-free analog front end for a capacitive touch sensor and less research effort is directed towards the digital back-end. This provides an interesting research opportunity to explore the issues associated with back-end of capacitive touch technology. One of the issues of interest is the scaling of capacitive electrode from a smart phone touch screen to a large wall mounted display. Figure 1.16 is a good representation of the two options pattern designer can deploy during the scaling process.

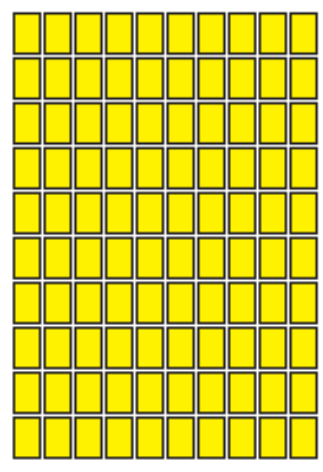

(a)

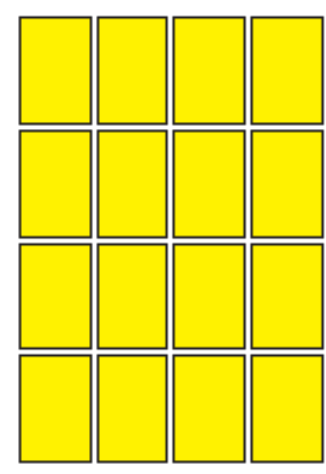

(b)

Figure 1.16: Capacitance electrode scaling techniques. (a) Scaling up by keeping electrode size same (b) Scaling up by increasing electrode size.

Going by the arrangement in Figure 1.16 (a), the microprocessor responsible for collecting touch signals from the physical sensors will have to scan a large number of sensors in order to successfully locate the finger. This implies that more than one microprocessor will be used in a multiplex arrangement which will increase the cost and real estate issues. If we decide to go by arrangement in Figure 1.16 (b) then we can work with only one microprocessor but our results will be inaccurate as now the basic element of the grid is scaled up and for good linearity tracking, the finger must cover more than one grid element in both row and column direction. If we follow a simple formula of scaling up the electrode size in the grid with the increase in area of touch panel, then we will end up with electrodes bigger than the size of 
normal human finger.

Another option is to add up all the charge present in all electrodes in a row and only send the sum to the controller, similar to what happens in self capacitance. But that, as mentioned earlier will diminish the multi-touch capability of the panel. Some of the current OEMs manufacturing large-scale capacitive touch screens tend to use self capacitance technique instead of mutual capacitance but it is not a viable solution as it does not support multi-touch [12].

Another interesting topic is the design (or shape) of the sensor itself. Each OEM is using their own patented designs in their products. It is not known which pattern is optimal in terms of accuracy and whether a methodology can be devised to compare different grid patterns. This is an exciting topic which will be addressed in detail in Chapter 3. Similarly, industry is using exhaustive sampling in existing designs, which is not an optimal solution as explained in Chapter 4. Interpolation techniques in place are also outdated and more accurate ones will be proposed in Chapter 5 .

During this research, different capacitive touch screen sensor patterns were studied and a methodology was devised to test these patterns for accuracy and precision. We looked at characteristics of capacitive touch signal and constructed a finger model to test our methodology. We experimented with various sub pixel interpolator algorithms during our work and proposed efficient and accurate sampling techniques for finger detection and tracking.

\subsubsection{Issues to Address}

Our investigation has provided us with these three research problems, that will be explored in detail in subsequent chapters.

1. Can we design a robust methodology to test different grid pattern designs in order to find the optimal pattern?

2. Can we transform a capacitive touch screen from small to large-scale panel without loss of precision or increase in controller cost? 
3. Can we propose an interpolation algorithm for capacitive touch panels which is based on finger model and has the least subpixel error?

One may ask the potential benefits from answering these questions. It is a known fact that retail market is shifting to high-tech touch enabled applications and thus, in future, a seamless user experience is the key for both manufacturers and customers. Capacitive touch technology already holds the market for smartphone and tablet touch screens and thus it is only certain that with time, it will dominate the touch panel market as well. As noted in section 1.1, the capacitive touch panel market is growing (0.22 million units in 2014 and 0.78 million units in 2019 [1]) but at a slower than expected rate. The reasons are the challenges faced by the industry in scaling up the touch panel without sacrificing the cost or precision. Answering these questions will help not only the industry but also retail sector and the consumer in getting the same smooth and precise touch experience from a retail touch panel which they get from their touch enabled smartphone device.

\subsection{Outline and Contribution}

This thesis investigates the issues raised in section 1.3.1 and proposes viable solutions that can be implemented in hardware.

In order to stress why research on accurate large-scale touch panels is important, we start our thesis with Chapter 2 which introduces a geo-visualization multi-touch portal developed in Nanyang Technological University (NTU). The portal uses a 55 inch infrared touch panel to visualize natural hazards data around the world. We discuss the impact of a large-scale touch panel on Earth science education and outreach using quantitative analysis and demonstrate through precision analysis why a capacitive touch panel will increase the usefulness of the system manifold.

Starting from Chapter 3, each issue listed in section 1.3.1 is tackled one by one. In Chapter 3, capacitive touch panel is introduced as an image processing problem and 
a methodology is devised for testing grid patterns. Multiple design concepts were implemented as binary images in Matlab and ANSYS, and a simulated robotic finger moved over the touch panel in various directions. Using Fourier transform, hybrid grid patterns are designed and simple geometrical techniques are proposed to make existing touch patterns more responsive. The goal is not to design an optimal grid pattern but to provide a methodology from which different patterns can be analyzed on image processing merits and techniques. The majority of the work presented in this chapter has already been published in [27] and [28].

In Chapter 4, we discuss the traditional sampling methods used in capacitive touch panels and their shortcomings. As the screen size becomes large, sampling becomes a problem because it takes time to measure capacitance at each capacitor on the panel. We refer to one of the traditional sampling methods i-e charge transfer technique and improve upon it by proposing multiple combinatorial algorithms in order to reduce the sampling time without sacrificing accuracy. The concept of greedy algorithms and compressed sensing is explored in depth with respect to capacitive touch panels. We propose an efficient sampling algorithm based on compressive sensing principle. We also observe that if the unique characteristics of capacitive touch panels are taken into account, a more efficient and accurate sampling algorithm can be formulated. We discuss the concept of pre-recovery sampling, which helps in reducing the sampling time even further. Using FLOPS analysis, it is shown that the proposed sampling procedures can outperform the traditional methods by a large margin. Some of the work presented in this chapter has been published in [29] and [30].

After sampling the signal, the third important aspect of capacitive touch panel (subpixel interpolation) is investigated in Chapter 5. Interpolation is important in order to find the accurate location of finger on the panel. We investigate various one dimensional subpixel interpolation techniques and observe their bias due to finger movement across the panel. After careful observation, a two dimensional Gaussian interpolation algorithm is proposed and it is shown that it is not only accurate but also efficient enough to be easily implemented in an embedded system application. 
The work presented in this chapter is published in [30] and [27].

The final chapter summarizes the work presented in this thesis and provides conclusion and closure. It also talks about possible future research directions on both academic and industrial level in order to solve up and coming important problems in the field of touch technology. 


\section{Chapter 2}

\section{Large-Scale Touch Panels and Earth Science Education}

\subsection{Introduction}

In Chapter 1, basics of various touch technologies were discussed, and based on market forecast and advantages of capacitive touch technology, it was concluded that capacitive touch panels will overcome resistive touch panel market share in years to come. However, the motivation of doing research on large-scale panels instead of small-scale touch screens is still not strong enough. It is already known that there are unanswered questions associated with transformation from small-scale touch panel to large-scale, but a bigger reason is required to dedicate this research to only big touch panels and this chapter does exactly that. It introduces a geo-visualization application called EOS-GeoTouch that is running on a large 55 inch infrared touch panel and is used to visualize natural hazards data around the world. The value of such a tool in education outreach and information dissemination is shown in this chapter as a means to understand the importance of doing research on design and development of large-scale capacitive touch panels, and why such technology has uses far beyond POS and industrial control systems. In this chapter, a link is established between the importance of large-scale touch panels and Earth science 
education using EOS-GeoTouch as an example. The current EOS-GeoTouch uses an infrared panel as it is the only commercially viable option at the present. Infrared panels although cheap, suffer from some serious problems. They cannot distinguish between user inputs and foreign objects like insects landing on the screen. Infrared panels can be accidentally activated when exposed to ambient light, a phenomenon more prominent if the panel is installed outdoors. Design-wise, they are aesthetically unappealing due to large protruding bezel all around the corners.

Despite all these issues, infrared panels are still extensively used due to their low cost. Capacitive touch panels, on the other hand do not suffer from the above mentioned issues. They are more precise and are unaffected by ambient lighting. They can work outdoors and indoors without any loss in precision [7].

Therefore, once it is established that large-scale touch panels are useful outreach tools, we perform a precision analysis to demonstrate that using a capacitive touch panel instead of an infrared one will only increase the usefulness of our system.

Use of multimedia in Earth science education is a well researched topic as indicated in $[31,32,33,34,35,36,37,38,39]$. However, the impact of large-scale touch panels on Earth science education is not covered in academic literature in a comprehensive manner. Research centers are developing multi-touch based visualization and interaction displays to disseminate information. ETH Value lab [40] is one such example of a collaborative research discussion environment situated at ETH Zrich. The Laboratory of Computational Science and Engineering (LCSE) at the University of Minnesota created an interactive visualization network to better understand mantle convection in 3D [41]. Their system consisted of a PowerWall display and interactive visualization software DSCVR. In [42], multi-touch display was used in designing a touch based 2D and 3D interactive dynamic exploration of Acoustic Doppler Current Profiler (ACDP) data sets. The Chinese Academy of Sciences (CAS) developed a Digital Earth Prototype System (DEPS) with the purpose of establishing a framework and model of digital Earth [43]. This three tier architectural model had spatial information about the surface data along with data 
coming in from various map servers around the world. The user interface layer was designed in a way that abstracted the hardware and the technical aspects of the software away from the user, making it intuitive to use.

Earth science education in state of the art institutions of higher learning has moved on from traditional learning materials that involve books, presentation slides and whiteboards to a more technological based approach. Use of Geographic Information Systems (GIS) as a teaching approach is common in modern research and teaching facilities. In [44], it is shown that the use of modern technology can have a positive impact on student performance and learning, and that these tools have, in certain cases, outperformed the traditional learning methods [45]. Today, there are several databases that can be readily incorporated in the teaching of Earth science. For example, the Smithsonian Institution (SI) for volcanoes and the United States Geological Survey (USGS) for earthquakes. This provides a great opportunity to develop and implement systems that can read into different formats of complex databases and visualize them in an easy to understand manner for students to see and interact with. Tablet devices provide superb interactivity, but are not as effective as very large-scale displays for handling multi-layered complex mapping applications.

Another use of large-scale panels is in increasing Spatial Visualization (SV) abilities of students. $\mathrm{SV}$ is the mental representation and manipulation of $2 \mathrm{D} / 3 \mathrm{D}$ shapes or 2D/3D topography. A 2010 report [46] identified SV as an important skill for student performance in STEM courses and as stated in [47], Earth science courses at school and college level help develop SV skills of the students. One of the major objectives behind developing and installing a large-scale touch screen based software is that such displays can provide a high sense of geographical spatial visualization. Also, touch based input helps in designing gesture based responses which are not only natural to the user but with the advent of touch based smart phones, easy to follow.

With massive data sets of natural hazards, it becomes important to create systems with user friendly touch experience. Large-scale, high resolution multi-touch panels 
are the solution to this problem. The large visual real estate provided by such displays combined with the usability of touch input ensures a learning experience not possible using small-screen computing systems.

\subsection{EOS-GeoTouch: Basic Architecture}

The EOS-GeoTouch project was launched in 2008 and the main purpose of this project was to build a multi-touch system for education, collaboration, public dissemination and discussion within Earth Observatory of Singapore (EOS) for geologists and Earth scientists [48]. As the scientists required a means of accessing accurate data quickly in a showcase location for dignitaries, press and other visitors, EOS-GeoTouch vision was to emerge as a useful tool to visualize GIS content on a large multi-touch screen.

Figure 2.1 shows the basic components of an individual EOS-GeoTouch system. The system uses a multi-touch infrared overlay as the primary display ( 55 inch). The touch panel is permanently attached as a frame on a smart TV. Both TV and the panel are connected to a laptop hidden in a cabinet behind the screen, responsible for running the EOS-GeoTouch software. The whole system is also connected to a local map server, which continuously feeds data to EOS-GeoTouch, both real time and static.

The touch panel uses a touch driver software from PQ labs for recognizing touch input [19]. This information is then sent to the higher application layer in the laptop via a USB connection where it is translated in a meaningful gesture using Microsoft surface API. The application layer then responds to the touch command and causes changes in map display. If the command is to fetch data from the servers, the application sends a query to the local server for map data which is then georeferenced and displayed. The whole system in use is shown in the Figure 2.2.

The software is built on a client-server model for obtaining and displaying data. The map and layers are stored in a local server and the PC running the software queries 


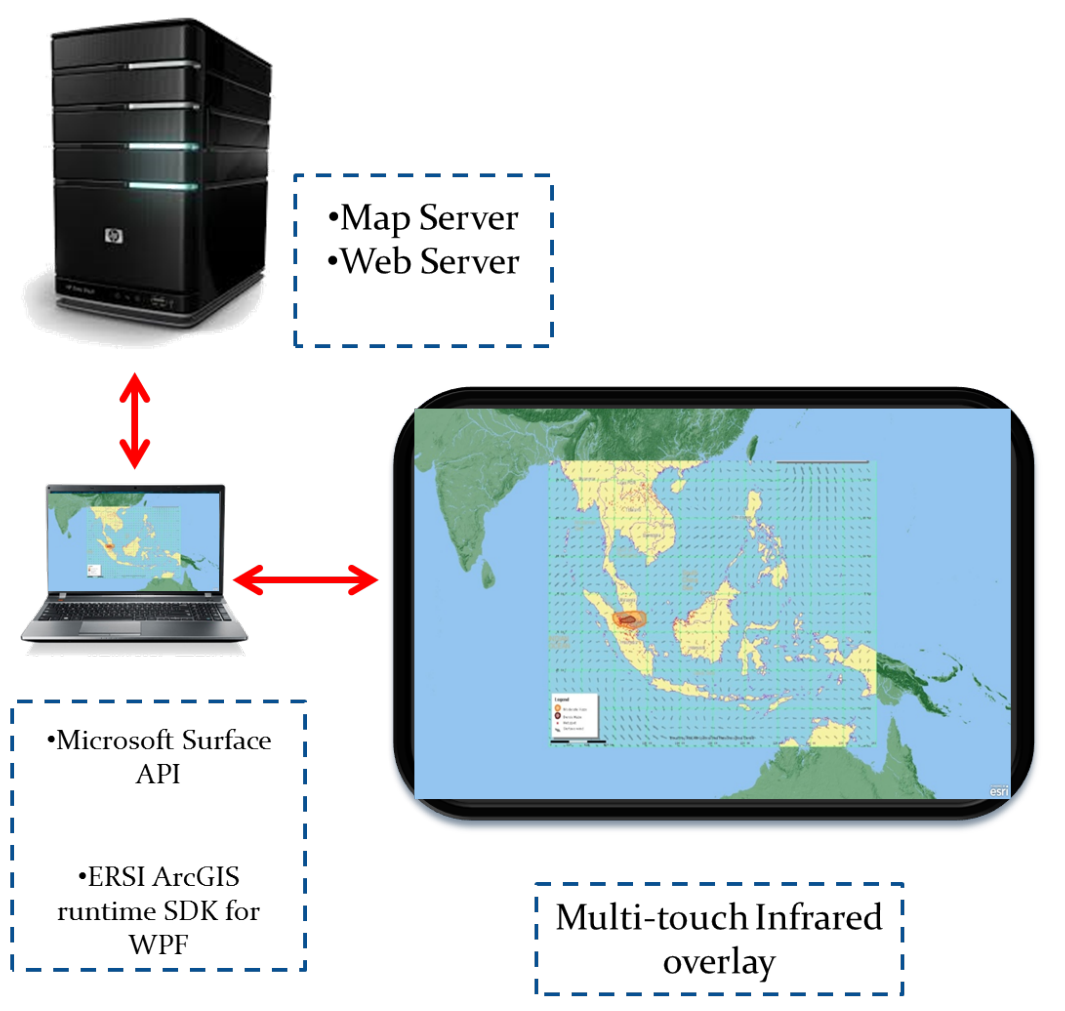

Figure 2.1: Basic components of an individual EOS-GeoTouch system. A large scale multi-touch overlay, a PC running the software and local map server and/or a web map server

the server for data and displays it on the touch screen. The server used is ArcGIS which is specifically designed by ESRI for hosting GIS datasets [49]. The software at the client side uses C\# libraries and ArcGIS SDK functions to query maps and layers constantly. EOS-GeoTouch was initially confined to the premises of Nanyang Technological University (NTU) because the server was a local machine, but later the data was transferred to a web server.

EOS-GeoTouch uses the Model-View-View-Model (MVVM) architecture which is specially designed for Microsoft Surface API applications. Figure 2.3 explains the basic flow of MVVM pattern.

As shown in Figure 2.4, the software architecture is built using a minimalistic approach. The View and the ViewModel are separate entities throughout the code and whenever a new feature (for example freehand drawing tool) is added, it is always divided into View (responsible for styling and display of the tool button) 


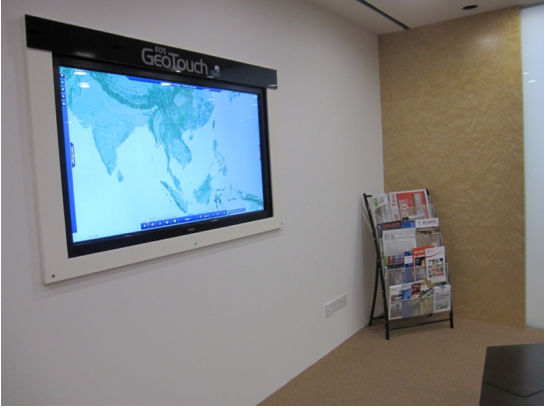

(a)

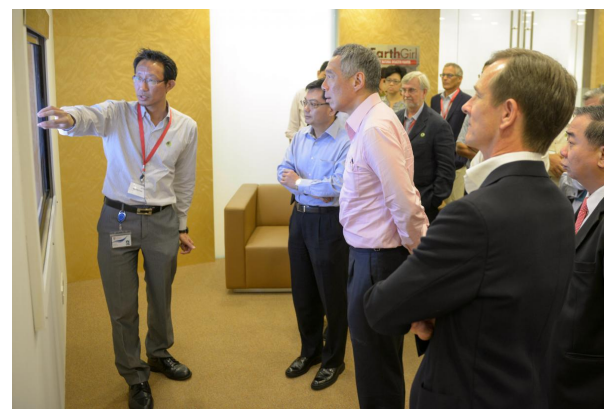

(b)

Figure 2.2: (a) The original EOS-GeoTouch just after being installed in EOS lobby (2009). (b) Using GeoTouch to give a presentation on climate change to minister for education and Prime Minister of Singapore (2014).

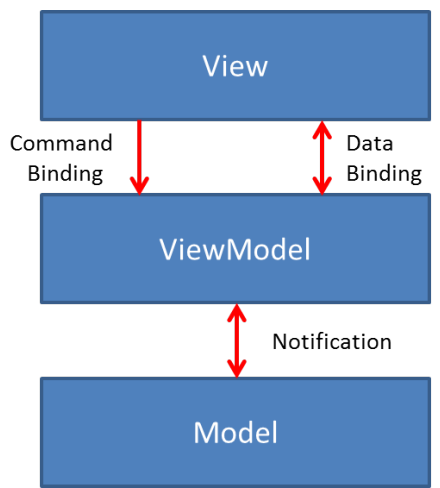

Figure 2.3: Basic Concept of a Model-View-View-Model System.

and ViewModel (responsible for the function to be run when the button is tapped). There is no User Interface (UI) specific logic in ViewModels throughout the code. A ViewModelLocator is used to bind Views to their respective ViewModels, whereas RelayCommands are used to call methods from Views to ViewModels. MVVM Light Messenger service is used to communicate between different ViewModels. EOSGeoTouch uses Extensible Application Markup Language (XAML) for the View files, C\# for the ViewModel files, and the ViewModelLocator for custom methods. Because EOS-GeoTouch system relies on an infrared panel, it is important to install it at a location with minimal direct lighting as infrared panels are susceptible to ambient light (More details on this problem at the end of this Chapter). The display is also located close to the power and network outlets for convenience. The original EOS-GeoTouch was installed in the EOS lobby where there is ample space to sit or 


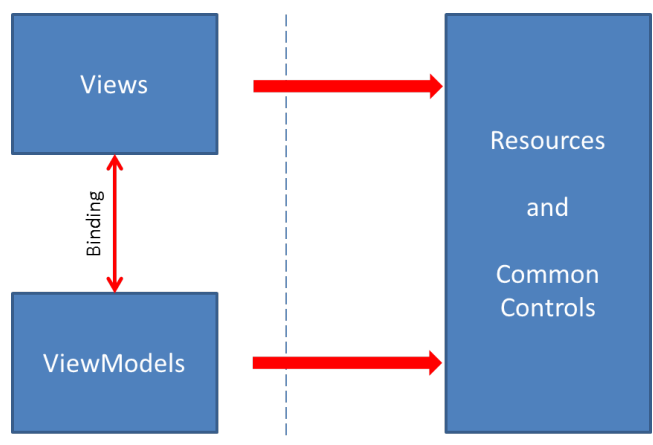

Figure 2.4: Simplistic software architecture of EOS-GeoTouch

stand in front of the screen. An informal survey showed that the majority of the EOS researchers were using EOS-GeoTouch during office hours, mainly for research discussions and project demonstrations to visitors. The system was used exclusively by principal investigators and senior researchers which led to the suggestion that perhaps additional stations would foster usage by students and the junior research staff.

\subsection{EOS-GeoTouch as an Education and Outreach Tool}

EOS-GeoTouch is a map visualization application and hence most of the screen space is reserved for the map itself (Figure 2.5). The tools are on the left and layers are on the right hand panels as shown in Figure 2.6. Let us briefly list the main features of EOS-GeoTouch.

Using the freehand draw tool shown in Figure 2.7(a), user can identify a region of interest. The freehand drawing precision depends upon the precision of the touch panel. EOS-GeoTouch has the ability to display otherwise difficult to understand databases and tables of earthquake and volcano information in an easy to understand manner. Using the Layers menu from the right hand panel, various topographic and seismic layers can be added on to the map and it is possible to adjust their transparency in order to better visualize the correlation between basemap and the 


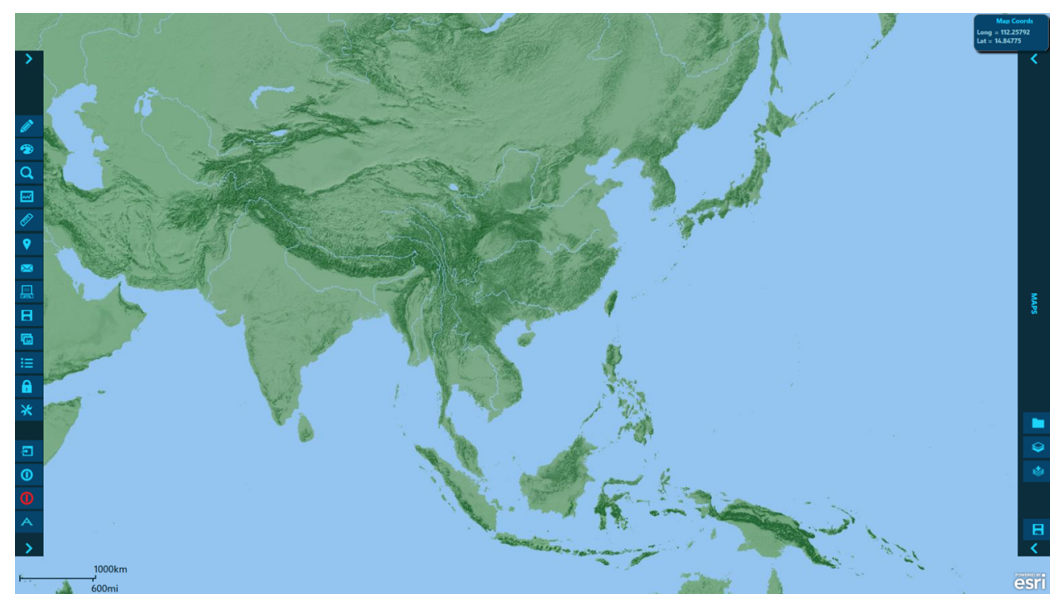

Figure 2.5: The main display of EOS-GeoTouch when turned on.

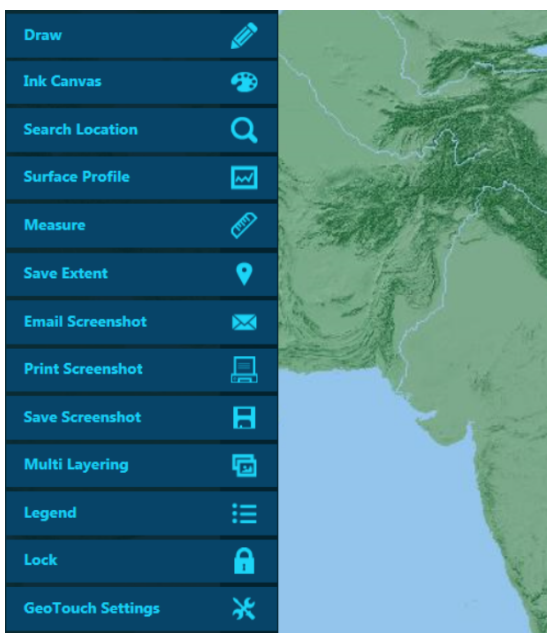

(a)

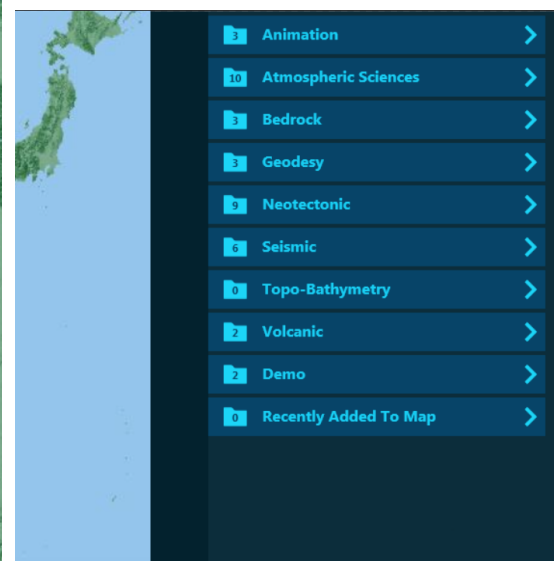

(b)

Figure 2.6: (a) Tools are on the left and (b) Data Layers are on the right hand side of the screen.

layer. As shown in Figure 2.7(b), bedrock layer from the Sumatra region has been added on top of the basemap with $50 \%$ transparency.

It is also possible to add point based layers, for-example volcanoes in South-East Asia, so that when the points are touched, relevant information about the volcano shows up in a pop-up window as seen in Figure 2.7(c). EOS-GeoTouch contains a wealth of information for those interested in Earth science. The data is divided into 4 major categories covering atmospheric, volcanic, seismic and structural geology research. The research done by EOS scientists on the recent major earthquakes (Nepal 
2015, Kota Kinabalu 2015) are also available. Database of historic earthquakes and typhoon tracks is provided as well. Other features include elevation profiling, cloud sharing and private layers access. The available data is regularly updated with new EOS research results or after significant events like a hurricane, an earthquake or a volcanic eruption. The focus normally remains in the South-East Asia region but global datasets of volcanoes and earthquake events are also available. The EOSGeoTouch system allows seamless communication between students and teachers. Both students and teachers can share maps with each other and upload from their personal accounts. This way, user submitted data from time to time further enriches the information available to students.

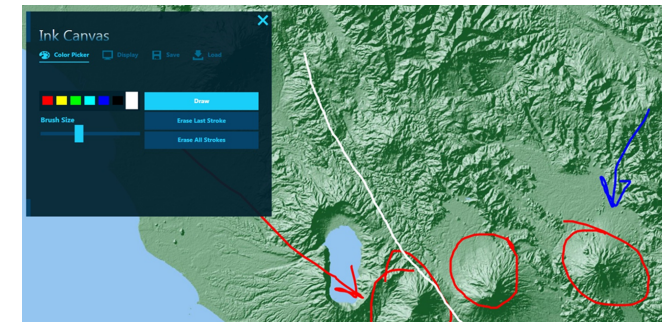

(a) Using the draw tool.

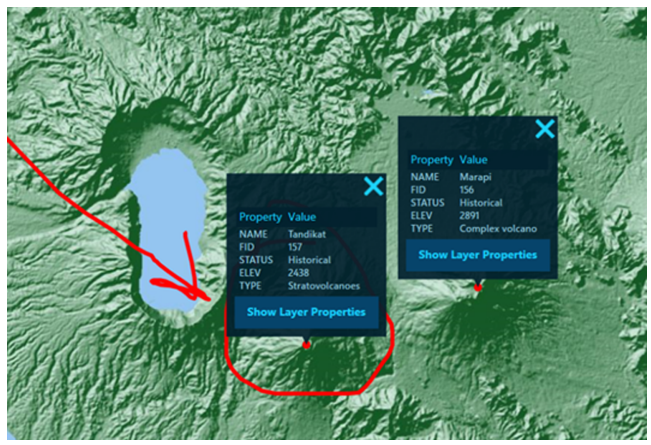

(c) Tapping the volcanoes to study their basic properties.

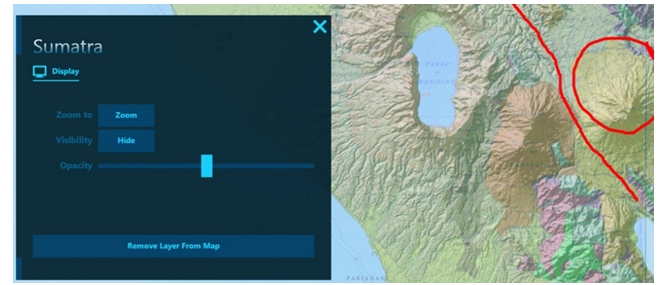

(b) Adding georeferenced layers on top of basemap.

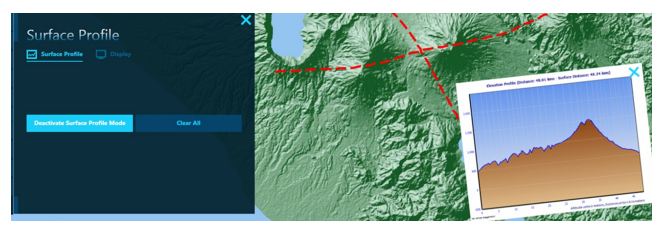

(d) Elevation profiling.

Figure 2.7: Different features of EOS-GeoTouch. 


\subsubsection{Quantitative Analysis of EOS-GeoTouch Impact as an Outreach Tool}

A quantitative measurement of the EOS-GeoTouch system usage can help to better understand its effectiveness and impact as an outreach and education tool at EOS. This section analyzes data gathered between January 2012 to July 2016.

\subsubsection{Objective}

We wish to understand the impact EOS-GeoTouch has had in the past 4 years in terms of being an effective outreach and education tool at EOS.

\subsubsection{Methodology}

The process of using EOS-GeoTouch in a presentation or discussion has three major components.

1. The presenter: one who is using EOS-GeoTouch for presenting a project or an idea.

2. The event: project demonstration, presentation, research discussion meeting or an undergraduate class.

3. The audience: can be students, teachers or scientists etc.

The methodology is to take all the events involving EOS-GeoTouch that happened in EOS from 2012 to 2016 and visualize them by following categories.

1. Category A: Where presenting on EOS-GeoTouch was only a part of the whole event.

2. Category B: Where all presentations at an event were done using EOS-GeoTouch. 
3. Category C: Where the audience interacted with EOS-GeoTouch on their own after the event.

The results are shown as a pie chart in Figure 2.8. We are also going to divide the audience with respect to the following categories and visualize the result in the form of another pie chart shown in Figure 2.9.

1. Students

2. Visitors with scientific background

3. Visitors with non-scientific background

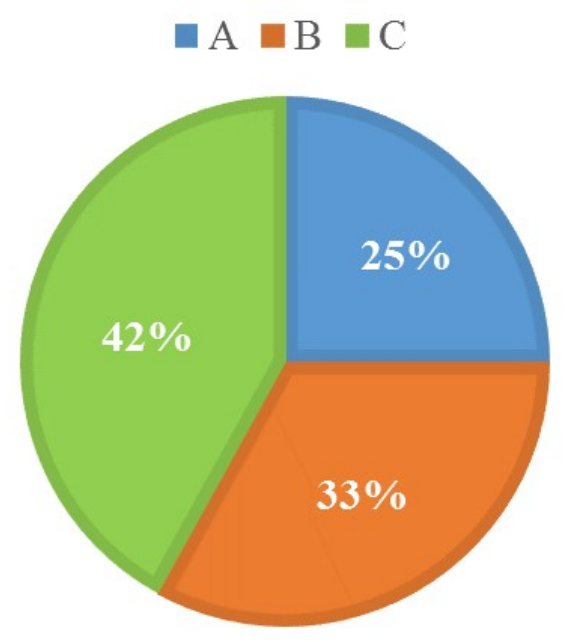

Figure 2.8: Pie chart representing the breakdown by categories of events in and outside EOS that used the EOS-GeoTouch system (Category A: 25\%, Category B: $33 \%$ and Category C: $42 \%$ ) (best viewed in color).

It is also important to divide events by year in order to understand how much attention EOS-GeoTouch got by the EOS scientists, students and teachers alike over the years (Figure 2.10). 
- Students

- Visitors with scientific background

घ Visitors with non-scientific background

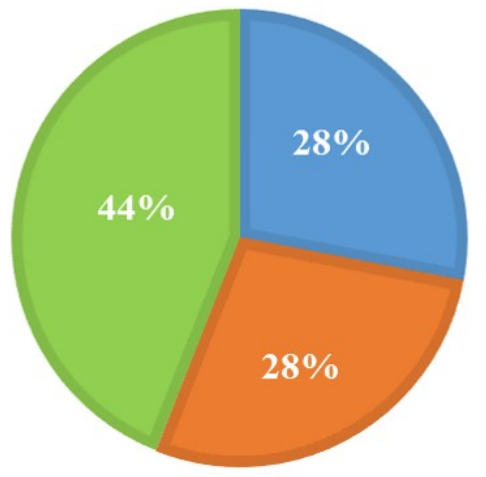

Figure 2.9: Pie chart representing the breakdown of events by audience type. (Events involving students: 28\%, events involving visitors with science background: $28 \%$ and events involving visitors with non scientific background: $44 \%$ ) (best viewed in color).

\subsubsection{Discussion}

From Figure 2.10 it can be seen that, EOS-GeoTouch has been increasingly utilized in both scientific and non-scientific events. In the first seven months of 2016, the total number of events involving EOS-GeoTouch is almost equal to the number of events for the whole 2015. The reason for this ascend can be found out by drawing a correlation between Figure 2.8 and Figure 2.9.

In approximately $42 \%$ of all events, the audience expressed likability to EOS-GeoTouch by interacting with the system and by asking details on how to create a similar map visualization display at their place of business. EOS-GeoTouch is more usable than other commercial Earth science visualization software, as evident from the fact that it was used to demonstrate complex geology research problems to a non scientific audience in $44 \%$ of all events. In Figure 2.11, a correlation is drawn between nonscientific audience and their level of interaction with EOS-GeoTouch after the event. More than $60 \%$ of the visitors were seen interacting with the system. As an outreach tool, EOS-GeoTouch goal has always been to create Earth science and geology interest in the common public by means of an interactive hardware and software. 


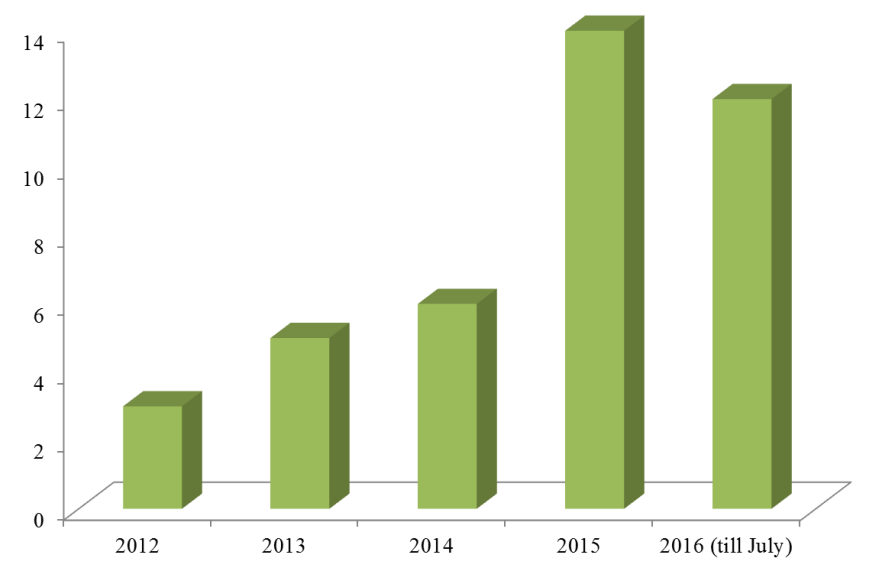

Figure 2.10: Chart representing number of events per year (2012 - July 2016).

- Visitors interacted with EOS-GeoTouch

- Visitors did not interact with EOS-GeoTouch

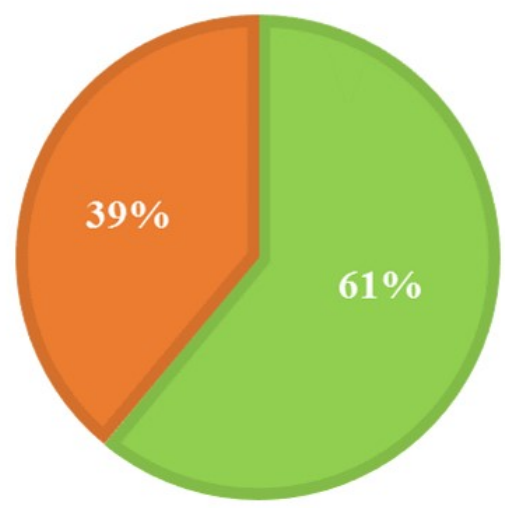

Figure 2.11: Pie chart showing the percentage of non scientific visitors who interacted with EOS-GeoTouch after an event (61\%) (figure best viewed in Color)

\subsection{EOS-GeoTouch and Urban Planning}

EOS-GeoTouch can be a useful tool for urban planners. Using the multilayer visualization tool (shown in Figure 2.12), urban planners have the ability to visualize different layers of data for the same geographical region. This functionality can help in decision-making about urban development in a particular region. Before and after comparisons can also be useful for observing the effects of tsunami, earthquake and volcanic damage. 


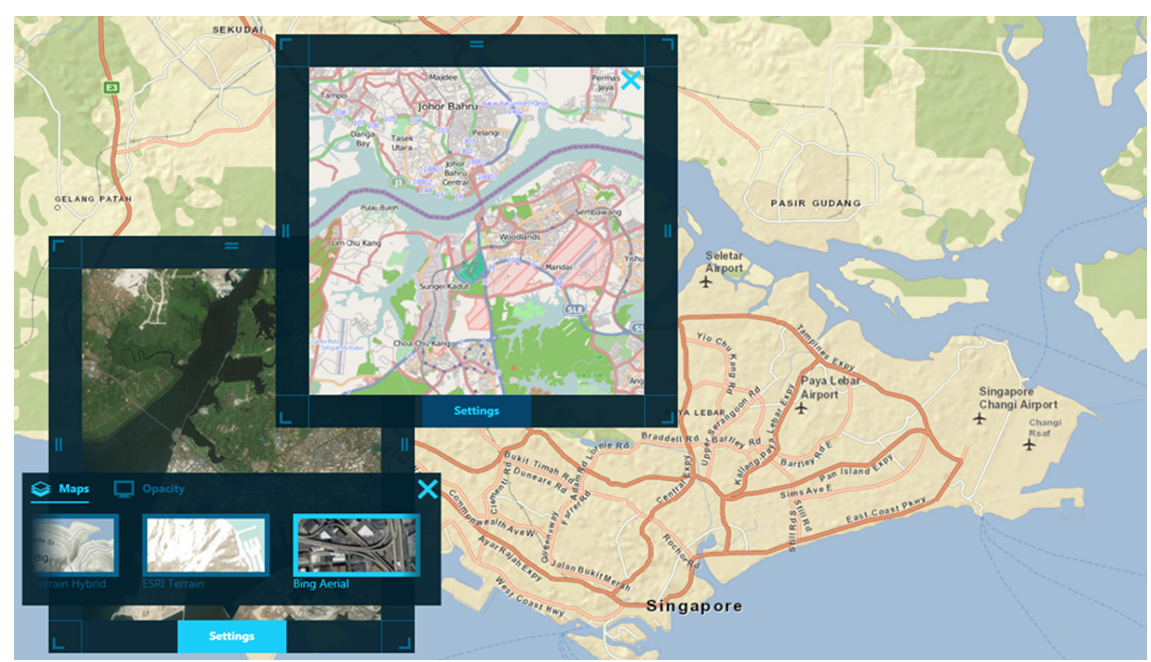

Figure 2.12: EOS-GeoTouch and urban planning using the multilayer visualization tool.

Urban planners using EOS-GeoTouch can also pose questions, such as 'what will happen if a volcano erupts in this region?' As mentioned before, EOS-GeoTouch has tools that allow users to put all kinds of overlays and animations on the basemap of their choice. Urban planners can also overlay visualization of power networks, transport grids and pollution data from industrial zones.

\subsection{Shortcomings of Infrared Touch Technology and Comparison with Capacitive Touch Tech- nology}

Large scale touch panels have following major applications:

1. Collaboration and Discussion

2. Point of sales (POS) systems and Kiosks

3. Control rooms dashboarding

4. Dissemination of knowledge for example in Earth Science Education. 
In this chapter, we focussed on one of the application i-e Earth science education as a motivation for study on large scale touch panels. Once it was established during the research that EOS Geotouch is a useful application for education and outreach, we tried to improve the hardware of the touch panel to make EOS-Geotouch more useful. However, we faced the following three problems.

Low Touch Precision Accurate path drawing was not possible due to false touches in the current hardware (infrared touch panel). However, touch precision can be improved if the software is hosted on capacitive touch panels.

A simple test for analyzing EOS-GeoTouch touch panel precision using its draw tool is devised. The test is simple: using our fingers, we draw some slow, steady lines across the screen and see the smoothness and straightness of the results. Figure 2.13 shows that the infrared panel is displaying its strokes in a jaggedly manner, prominent when zoomed in (Finger 2.13(b)) and this causes some precision to be lost.

No bezier curve algorithm was used to smoothen the strokes and only the raw data reported by the touch panel API was used to draw the stroke. As reported in data sheet for PQ labs G4 series 55 inch multi-touch panel (similar to the one used in EOS-GeoTouch), the finger touch accuracy is $2.7 \mathrm{~mm}$ [50]. This accuracy is lower than Microsoft's 82 inch Perceptive Pixel (PPI) touch panel accuracy which is less than $0.036 \mathrm{~mm}$ according to their data sheet [51].

Limited Feature Development Scope due to non-availability of 3D touch. On the other hand, capacitive touch technology supports 3D touch thus it is useful for introducing additional gestures in the application.

Limitations in Use because of false touch reporting under direct sunlight. The EOS-GeoTouch system is installed indoors hence it is not directly affected by sunlight, but in future, if it is decided to install such systems outdoors, the current installations will start to report false touches. Recently, Peau Productions [6] tested infrared touch panels in varying light conditions and concluded that the touch panels do not work under direct sunlight. Referring to some screenshots of their experiment 


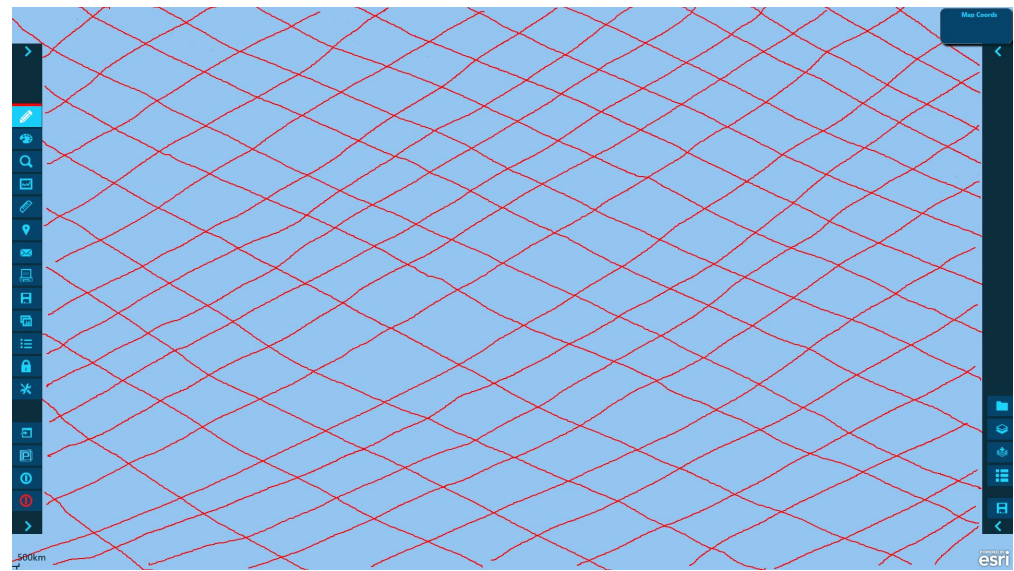

(a)

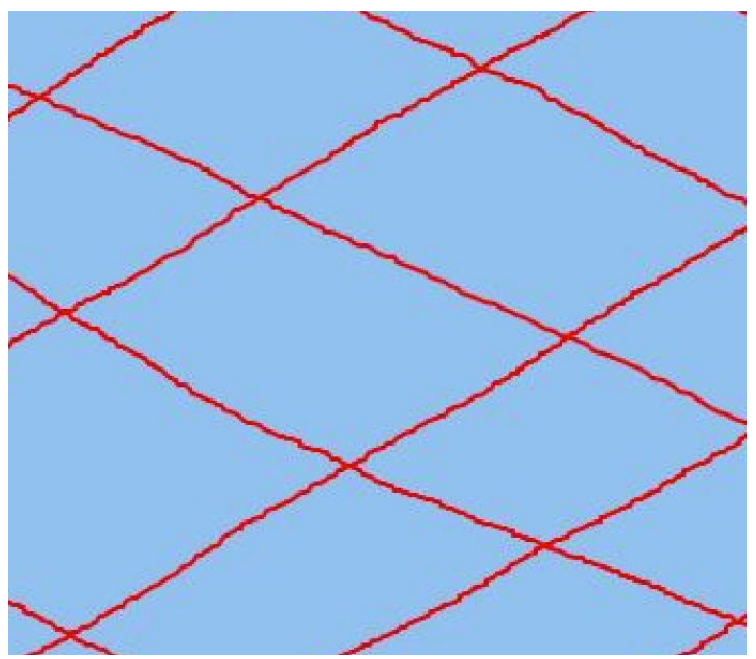

(b)

Figure 2.13: EOS-GeoTouch touch screen analysis results. The zoomed in shot (b) shows that the finger tracking accuracy is off by some margin.

video in Figure 2.14, it is obvious that the panel is reporting false touches during a finger tracking test. The false touches have been highlighted in the figure so that it is easier for the reader to notice them. Capacitive touch panels, as mentioned in Chapter 1, do not suffer from ambient light and thus are perfect for outdoor installation.

In summary, user experience of a useful and proven application like EOS-GeoTouch can be improved if it were to be hosted on capacitive touch panels. The need of the hour is to propose solutions for issues that are making it difficult for the industry to produce cost-effective capacitive touch panels. Therefore, in the next chapters, we 


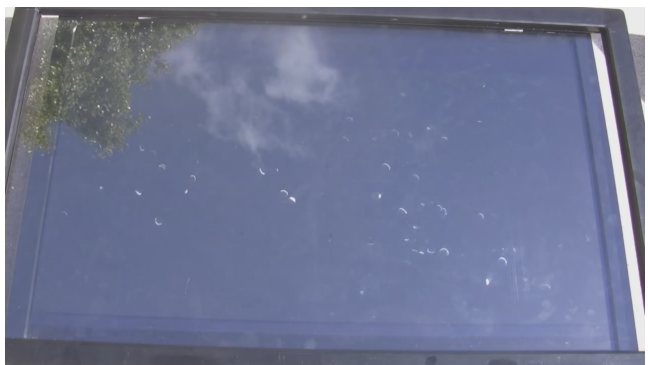

(a)

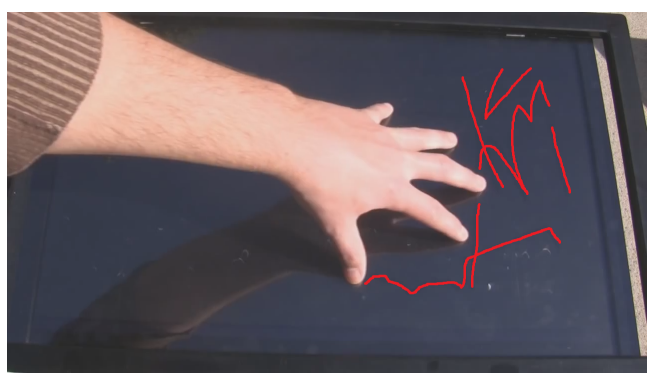

(c)

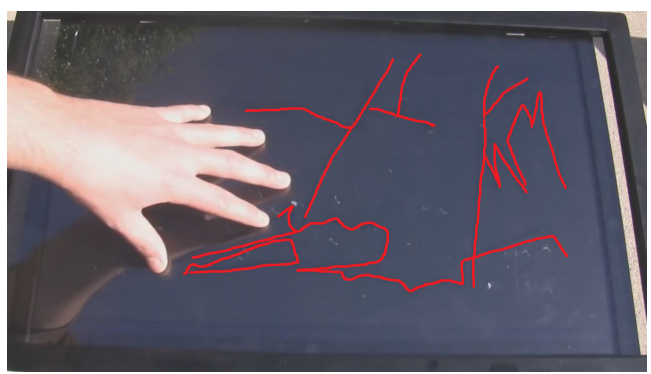

(e)

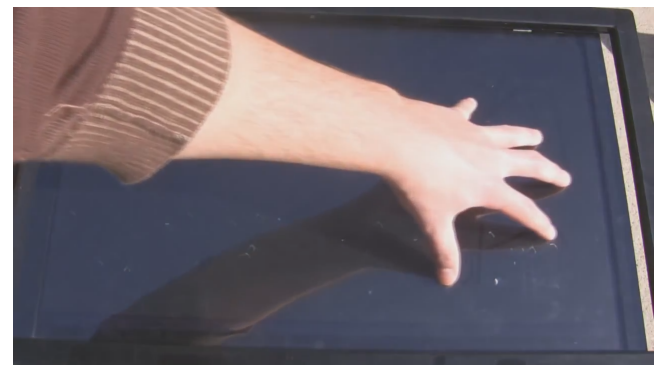

(b)

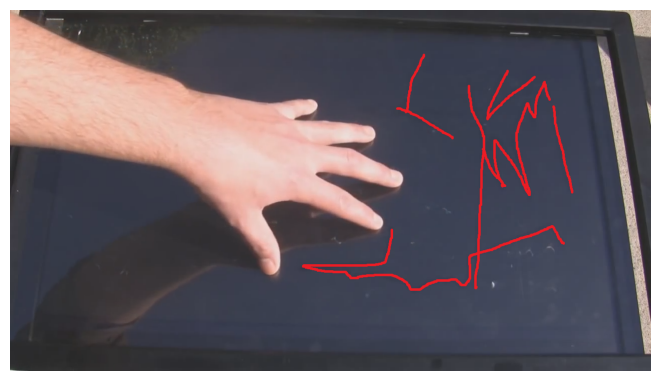

(d)

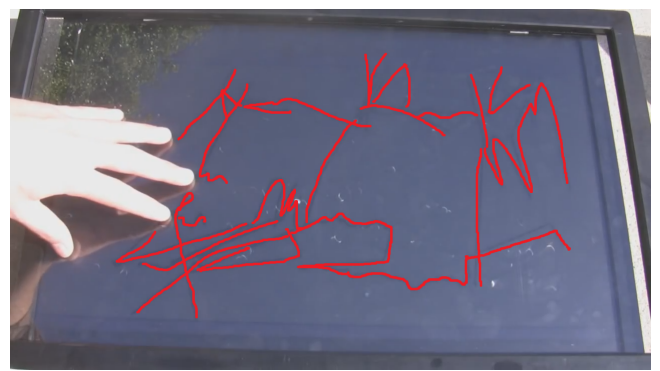

(f)

Figure 2.14: Peau Productions infrared touch panel ambient light tests. Five fingers were moved in a straight horizontal line from right to left. The touch points reported are highlighted in red. Screen grabs from [6]. (best viewed in color)

address various research problems involving design of large scale capacitive touch panels. 


\section{Chapter 3}

\section{Grid Pattern Design and Testing}

\subsection{Introduction and Related Work}

With the rise in penetration of capacitive touch screens into every aspect of our lives, there is an increasing demand of large sized touch screens for various applications in the market. Cost of manufacturing capacitive touch sensing devices is still greater than optical based and resistive devices. Because the device must be able to sense very small changes in capacitance (few Femtofarads $\left[10^{-15} \mathrm{~F}\right]$ ), the aim of a designer should be to find out the optimal point between sensitivity of the sensor and its immunity to noise [26]. The first issue to be addressed in this thesis is related to the physical design of the capacitive sensor (or electrode). This chapter probes into grid pattern design of capacitive touch panels and provides a methodology to compare the performance (accuracy) of touch panels so that an optimal design pattern can be constructed.

\subsubsection{Touch Panel Stack}

A typical touch enabled panel has a stack of several layers, which varies with the type of application and also depends upon cost and width of the touch panel. The common layers used are shown in Figure 3.1. 


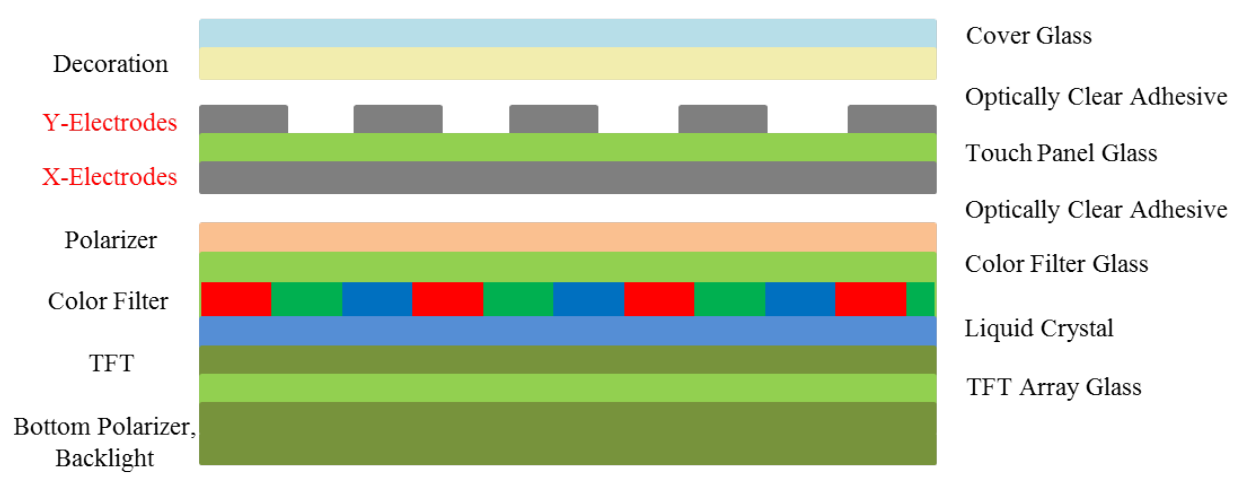

Figure 3.1: Typical stack for a touch enabled panel. Figure reproduced from [7] with changes.

Figure 3.1 shows the typical stack for a touch panel. A decorative coating under the cover glass provides protection to the layers beneath. The touch panel is attached to the cover glass and polarizer using an optical clear adhesive.

The arrangement shown above is the add-on type in which the touch panel is directly below the cover glass. The touch panel itself consists of a grid with driving (Yelectrodes) and sensing (X-electrodes) lines made from a conducting material. A rectangular grid pattern with $\mathrm{X}$ and $\mathrm{Y}$ electrodes perpendicular to each other is shown in Figure 3.1, forming intersections for charge storage. The color filter and its glass rests above the Thin-Film-Transistor (TFT) array. The bottom polarizer and back light are at the bottom of the arrangement.

Different companies use variations of traditional stackup in their products. Two major variations are sensor-on-lens and on-cell variation. These are shown in Figure 3.2. In a sensor-on-lens variation, the touch panel electrodes are moved to the bottom of the cover glass thus eliminating the need of touch panel glass and making the panel lighter and thinner. In an on-cell solution, the touch electrodes are moved to the bottom, right on top of the color filter glass, also resulting in a thinner panel as the touch panel glass is discarded. No matter what the arrangement is, the working principle is the same. 


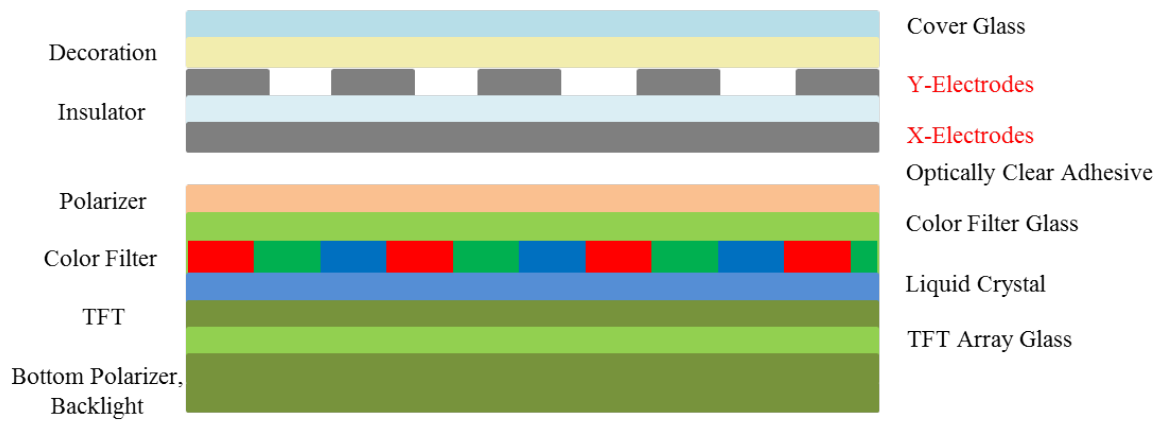

(a)

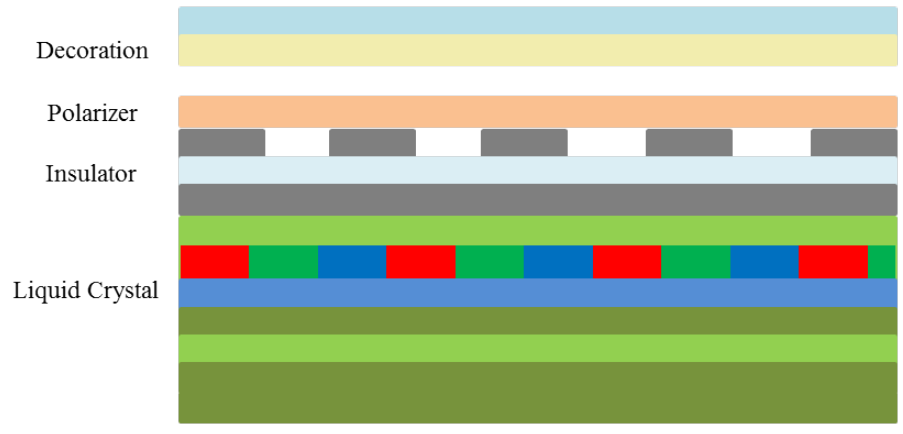

Cover Glass

Y-Electrodes

X-Electrodes

Color Filter

TFT

Bottom Polarizer,

Backlight

(b)

Figure 3.2: (a) Sensor on lens stack (b) On-cell stack. Figure reproduced from [7] with changes.

\subsubsection{Touch Panel Sensor Pattern Design}

Although a tremendous amount of research has been done in the field of capacitive touch technology, there is very little academic literature available on design of grid patterns for capacitive touch screens. Most of the material exists in the form of patents. Companies such as Avago, Synaptics etc. have been designing grid patterns for touch screens and track-pads for more than 15 years now [52, 53]. Krein and Meadows [54] addressed the issue of optimizing a capacitive panel so as to make it inexpensive and accurate while retaining immunity from electromagnetic interference from the TFT-LCD. They used a quasi-static electric field and applied it to the coating on the panel surface, thus making the computed position independent of coating resistivity. Ruan, Chao and Chen [55] proposed a multi-touch capacitive interface for medium sized touch panels (more than 12in). They used a low 
disturbance array cell, constructed using four MOSFETs in their sensing circuit in order to eliminate the noise effect from the LCD underneath the touch panel. Tai, Chiu and Chou [56] overcame the same issue of noise reduction by using a single conventional transistor in the touch panel circuit. In order to improve handwriting recognition in capacitive touch screen smart phones, Lin et al. [57] used a passive 3D stylus in order to measure $x, y$-directional position and $z$-directional strength during handwriting recognition. Baharav and Kakarala [58] explained the working of touch sensing mechanism of a diamond shaped grid pattern by simulation of a Gaussian finger on a touch panel. Expanding on their work, we present a methodology to evaluate the performance of grid patterns and study their Fourier transform plots in order to gain insight into what makes one pattern perform better than the other. A preliminary version of this work was presented in [28] and a technical extension has been published in [27].

The most common pattern is the diamond pattern shown in Figure 3.3. Here, the capacitors are neatly arranged in a diamond like structure and are spread over two layers, the upper layer forms the column layer and below it, is the row layer. The row and columns are not fused together and provide separate readings of the finger coordinates which is then used by the processor to determine the location of the touch.

Besides the diamond pattern, there are a number of grid patterns proposed by designers over the years. One of these patterns is the interleaved pattern proposed by Harley [52]. The basic element of the interleaved pattern is shown in Figure 3.4(b). After repeating the basic element along $\mathrm{x}$ and $\mathrm{y}$ axis, the resultant full scale touch screen panel is shown below in Figure 3.4(h). A similar design was proposed in [59] for use in laptop touch pads.

Mackey [53] patented a grid design for capacitive sensing where the shape of the basic element looks like two angled crosses as shown in Figure 3.4(c). However it should be noted that this is not the final form of the angled cross pattern. As the inventor suggests, this pattern should not be limited to this shape only but the 


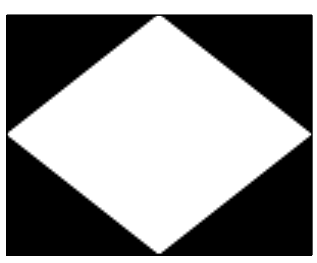

(a)

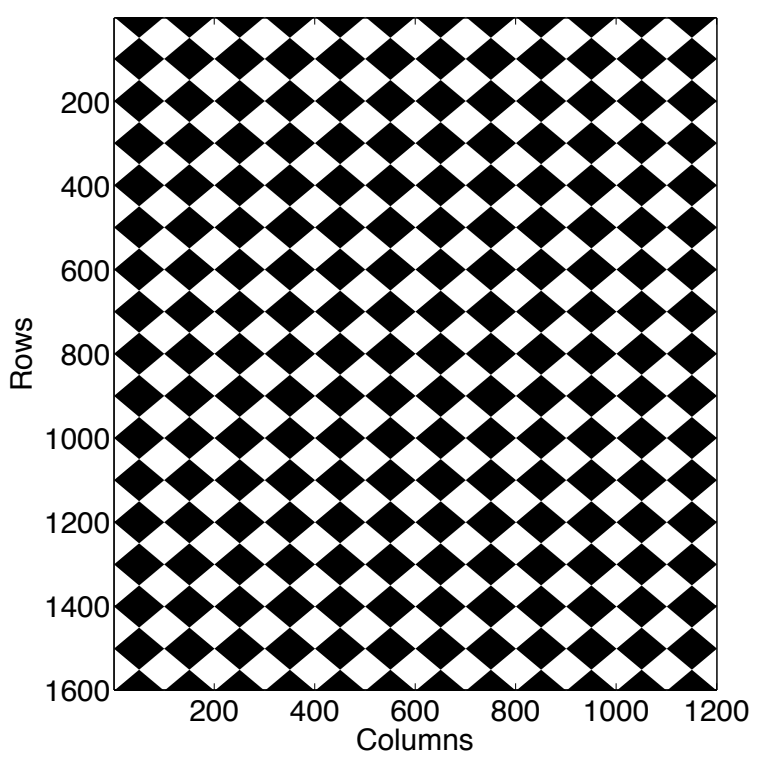

(b)

Figure 3.3: Diamond shaped (a) basic element and (b) grid pattern for capacitive touch panels.

pattern can be suitably varied while fitting in the basic shape of an angled cross. A $16 \times 12$ touch panel is obtained by repeating the angled cross along $\mathrm{x}$ and $\mathrm{y}$ axis as shown in Figure 3.4(i).

There are patents that propose a hybrid of diamond and interleaved patterns [60]. The basic element of one such design is shown in Figure 3.4(d) and is labeled Diamond-Interleaved for reference. From the patent, one cannot tell what advantage such a design can have over traditional interleaved or diamond pattern, thus strengthening the case for a methodology on testing capacitive grid designs.

It is not possible to review every single patented capacitive sensor design in this chapter as there are numerous patented designs $[61,62,63]$ but we would like to 
include one more pattern proposed by Ritter et al. [64], which is shown in Figure 3.4(e). This pattern can be labeled as double $\mathrm{T}$ pattern and it is designed in a way to take advantage of the fringing electric fields accumulated around the corners of the $\mathrm{T}$. One variation of double $\mathrm{T}$ can be an octagon-end grid pattern (shown in Figure 3.4(f)) and is labeled as modified double $\mathrm{T}$ for reference.

In order to determine finger position, the touch processor needs to get readings from both row and column layer. Therefore it first scans the rows and then the columns to get both $\mathrm{x}$ and $\mathrm{y}$ data which is then passed through an estimation algorithm to determine the location of finger on the screen. As a rule of thumb, the finger area must occupy at least one grid element (row and column both) at a given time in order to get a meaningful reading. If the grid basic element is greater than the finger diameter itself, it is difficult for the processor to calculate an accurate result. Also if the grid element size is reduced in order to get better accuracy, the designer will still end up with a lot of pin outs for even a smaller sized touch screen. Therefore the research problem is formulated as follows:

- The academic literature is limited when it comes to capacitive touch grid pattern design and comparison. All the information available is published as patents. Without any comparison methodology, it is impossible to justify what kind of grid pattern design is suitable for a particular application.

- Also, given a particular capacitive grid pattern, it is not known on how to improve its sensitivity or accuracy. In order to address the problem, it is important to compare and contrast various grid patterns for tracking accuracy and sensitivity.

It will be an interesting approach to go about changing the shape of a capacitive grid pattern basic element so that the new pattern is capable of sensing the finger more accurately even if the size of a basic element is the same or even smaller than the area occupied by the finger. An experiment to investigate this phenomenon is presented in Section 3.2. 

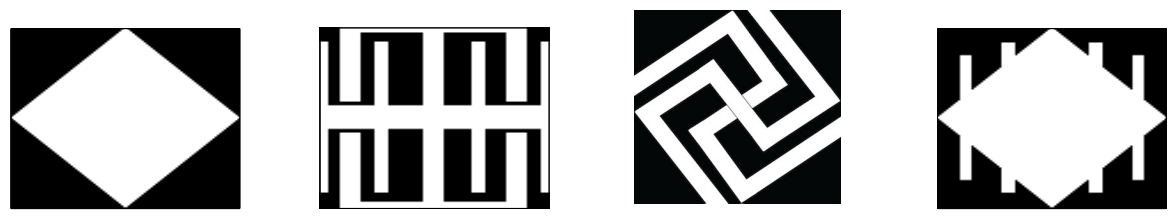

(a) Diamond grid (b) Interleaved grid (c) Angled cross (d) Diamond-
basic element.
basic element.
grid basic element
Interleaved grid basic element.
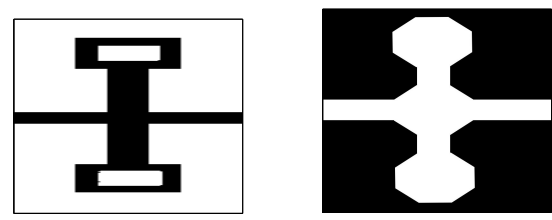

(e) Double $\mathrm{T}$ grid (f) Modified Doubasic element. ble T grid basic element

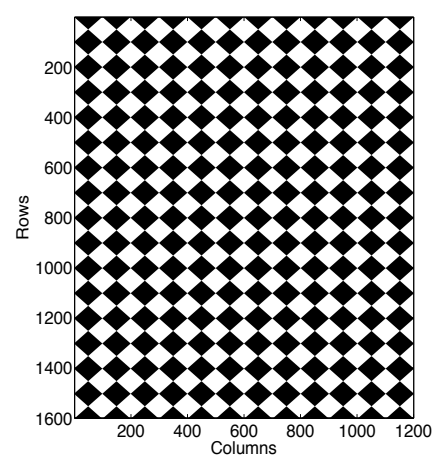

(g) Diamond grid panel

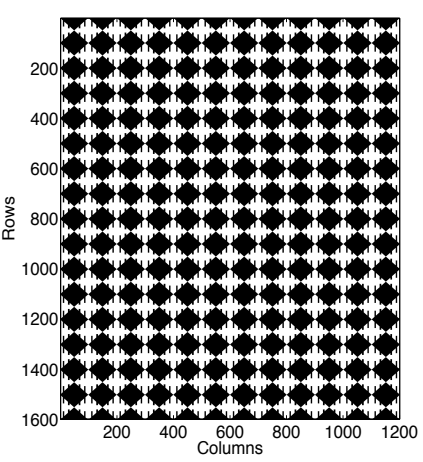

(j) Diamond-Antenna grid panel

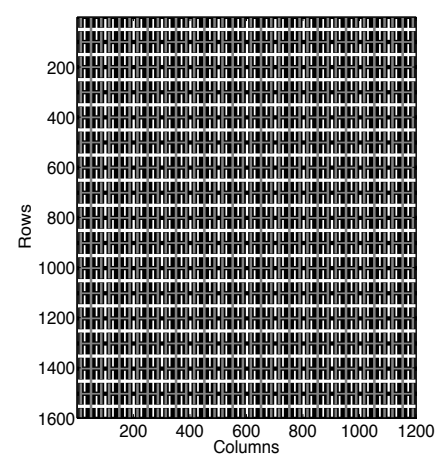

(h) Interleaved grid panel

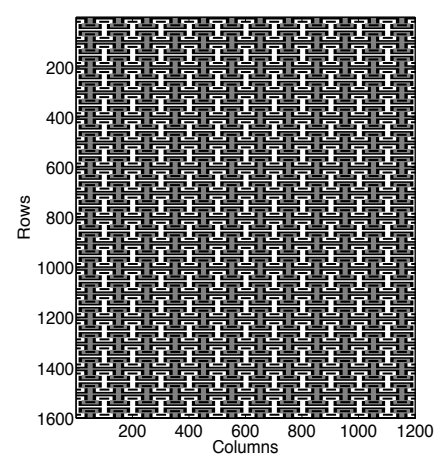

(k) Double T grid panel

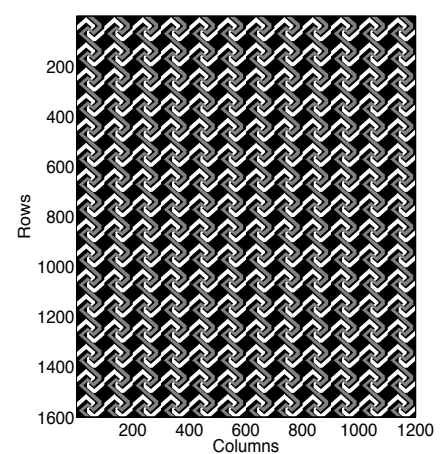

(i) Angled cross grid panel

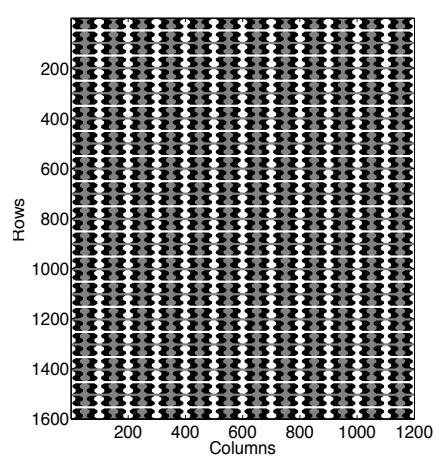

(l) Modified Double T grid panel

Figure 3.4: Grid basic elements (a)-(f) and their respective patterns (g)-(l) formed by replicating the basic element in horizontal and vertical direction. 


\subsection{Sensor Pattern Testing Methodology}

In order to address the problem of providing a methodology for calculating and comparing accuracy of grid patterns, all six grid patterns were designed in Matlab and were passed through a series of tests in order to determine their tracking accuracy. The panels used in these simulations have 16 rows and 12 columns each. The finger used is shown in Figure 3.5 and has a diameter of $15 \mathrm{~mm}$. The assumption here is that a finger can be represented by a 2-D Gaussian function. This assumption is not far fetched as previous research in this field has used such a profile in their simulations $[58,65,66,67]$. This assumption was also established based on our personal communications with touch panel industry during the course of this research, where it was observed that the set of measurements reported by the touch controller in a typical testing environment appears substantially Gaussian-shaped. Typical dimensions for the row and column spacing (and size) is about $5 \mathrm{~mm}$ in touch panels, also called pitch of the panel [58]. A single sensor in our experiments is made up of 100 pixels except for the double $\mathrm{T}$ and modified double $\mathrm{T}$ pattern. Taking the basic element of size 100 pixels will result in a $50 \mu \mathrm{m}$ sized single pixel.

There is no correlation between the overall size of panel and the diameter of finger. The proposed methodology and the results given in this chapter are not dependant on the size of the panel. The simulated finger movements on the panel are designed to be always contained with-in the total area of the panel. The reason for choosing a 16 by 12 grid was that 4:3 ratio for sensing and driving lines is most common in smart phones (iPhone 5 for example has a 4:3 ratio for its driving and sensing lines).

After getting the touch sensor panels and finger ready, it is quite a straight forward method to find out the amount of area covered by the finger. It can be done by placing the finger on the panel at a known location (see Figure 3.5 (b)) and then calculating the increase in capacitance for all the rows and columns one by one. The rows which are covered by the finger give much higher values as compared to 


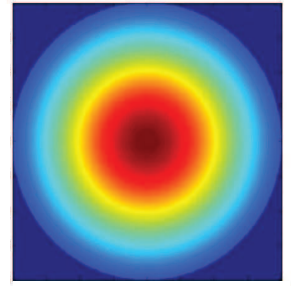

(a)

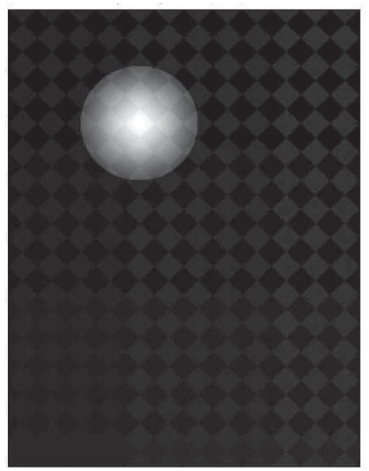

(b)

Figure 3.5: (a) Simulated finger profile used in the simulations (b) Finger superimposed on diamond grid. The columns and rows covered by the round finger are highlighted.

the other rows. These values are sent to an interpolation algorithm to estimate the location of the finger.

The response of estimators on all six patterns was tested when the finger was moved in diagonal, circular and in vertical direction, thus covering all the possible angles of motion. These tests are explained one by one below.

The diagonal motion experiment was also performed with multiplicative noise. The main sources of noise are from the LCD display and the charger. As LCD display is tightly coupled with the touchscreen sensor, it can often produce high noise levels (sometimes up to 10V). These noise levels are greater than the finger signal [68]. Similarly, charger noise can exceed touch signals by a large factor. These two sources of noise when combined, produce wide range, random and time-varying noise, making accurate noise modeling difficult. In Figure 3.6 (a) the readings collected by the touch processor in three rows are labeled as $x_{0}, x_{1}$ and $x_{2}$. The true peak will lie 
somewhere between $x_{1}$ and $x_{2}$ and is found by applying an interpolation algorithm. However after addition of noise, the readings change as shown in Figure 3.6 (b) thus making $x_{2}$ larger than $x_{1}$ and in effect changing the peak location. The interpolator has no way of knowing the original maximum value $x_{1}$ and will report inaccurate finger coordinates.

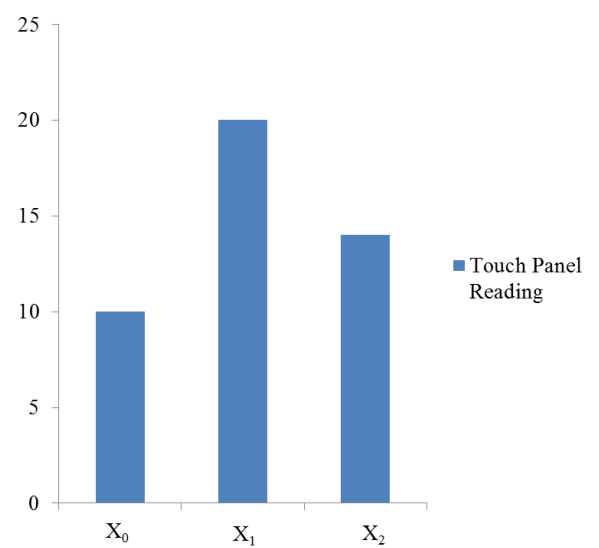

(a)

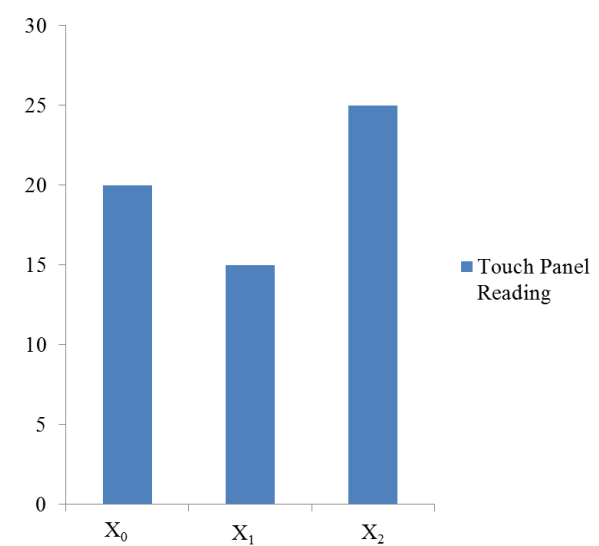

(b)

Figure 3.6: (a) Touch controller readings with zero noise (b) Touch controller readings with added noise.

\subsubsection{Diagonal Movement}

The finger was placed at a known location on all panels and dragged diagonally towards the center of the panel. On every iteration the finger was dragged to another known location on the panel and for each location the algorithm computed the new estimated position. Mean tracking error was calculated by finding the Euclidean distance between the true and the estimated location for each iteration.

The interpolator used in these simulations is Center of Mass (COM) interpolator, which uses three values as input and assumes that the spread of the inputs conform to a Gaussian distribution. The interpolator is just a weighted-average procedure and can be written as:

$$
\Delta r=\frac{r_{i+1}-r_{i-1}}{r_{i-1}+r_{i}+r_{i+1}}
$$


where the maximum value of the row reading is denoted by $r_{i}$, whereas $r_{i-1}$ and $r_{i+1}$ indicate the reading in the row below and above the maximum respectively.

Figure 3.8 shows the response of simulating the finger traveling along a straight line at $45^{\circ}$. Random multiplicative noise with amplitude of 0.3 is added to the signal. In Figure 3.8 and also for the subsequent Figures 3.9 - 3.10, the color code is as follows: Blue is the exact route and Red represents the estimated route using COM interpolator. The mean estimation error for each pattern is given in Table 3.1 with the smallest value highlighted in bold. It can be observed that:

- Interleaved pattern performs better than the other patterns even with reduced finger diameter.

- Angled cross pattern has the overall worst performance.

- The error for COM increases by a large margin with noisy signal.

Figure 3.7 shows the median, maximum and minimum tracking error along with mean error for diagonal movement. The conclusion still remains the same that the interleaved pattern has the overall least tracking error out of the six patterns.

Table 3.1: Diagonal movement tracking error comparison. Smallest error value is shown in bold.

\begin{tabular}{|c|c|c|c|c|c|c|}
\hline \multicolumn{7}{|c|}{ Diagonal movement tracking error with finger radius $7.5 \mathrm{~mm}$ and no noise } \\
\hline $\begin{array}{l}\text { Interpolator } \\
\text { COM }\end{array}$ & $\begin{array}{l}\text { Interleaved } \\
\text { Mean }(\mathrm{mm}) \\
\mathbf{0 . 6 1 4 9}\end{array}$ & $\begin{array}{l}\text { Diamond } \\
\text { Mean }(\mathrm{mm}) \\
0.6729\end{array}$ & $\begin{array}{c}\text { Angled Cross } \\
\text { Mean }(\mathrm{mm}) \\
0.7610\end{array}$ & $\begin{array}{c}\text { Diamond-Interleaved } \\
\text { Mean }(\mathrm{mm}) \\
0.6722\end{array}$ & $\begin{array}{c}\text { Double T } \\
\text { Mean }(\mathrm{mm}) \\
0.6880\end{array}$ & $\begin{array}{c}\text { Modified double } \mathbf{T} \\
\text { Mean }(\mathrm{mm}) \\
0.6737\end{array}$ \\
\hline \multicolumn{7}{|c|}{ Diagonal movement tracking error with finger radius $7.5 \mathrm{~mm}$ with added noise (noise amplitude: 0.3 ) } \\
\hline COM & 0.9716 & 0.9921 & 1.1599 & 1.0555 & 1.0525 & 0.9948 \\
\hline \multicolumn{7}{|c|}{ Diagonal movement tracking error with finger radius $5.0 \mathrm{~mm}$ and no noise } \\
\hline COM & 1.0268 & 1.1819 & 1.1792 & 1.1849 & 1.1567 & 1.1721 \\
\hline
\end{tabular}

\subsubsection{Circular Movement}

As the diagonal movement only accounts for a $45^{\circ}$ angle, it was necessary to check if the touch panels perform well with other angles. The finger was moved in a circular 


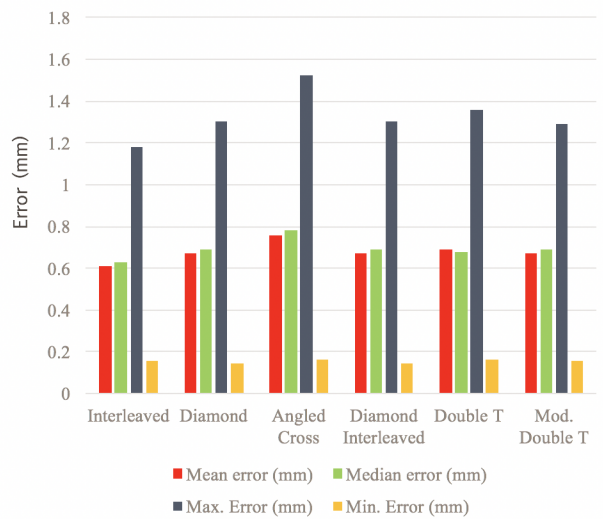

(a)

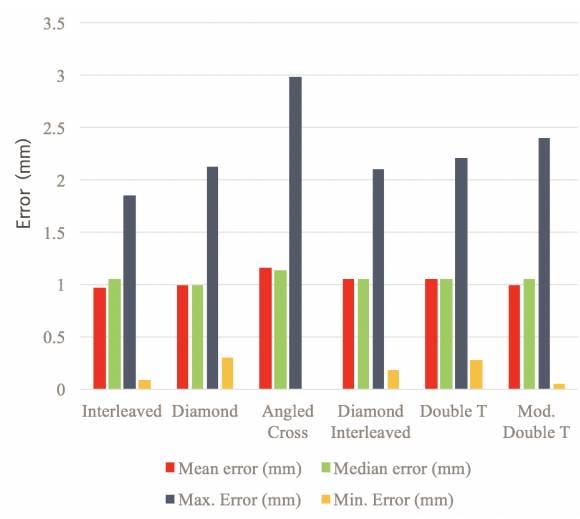

(b)

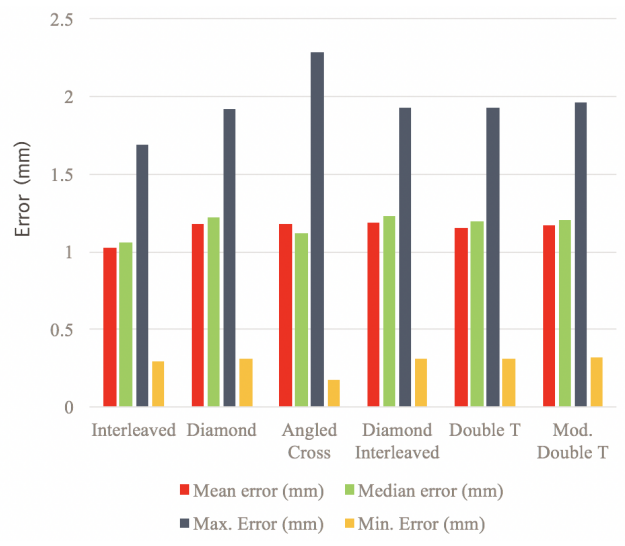

(c)

Figure 3.7: (a) Diagonal movement tracking error in $\mathrm{mm}$ with finger radius $7.5 \mathrm{~mm}$ and no noise (b) with additional noise (c) Finger radius $5 \mathrm{~mm}$ with zero noise [In color]

motion and the tracking estimator results are shown in Figure 3.9. The mean error for COM can be viewed from Table 3.2. The difference in performance is not clear from the figure but as seen from the table, interleaved pattern has less tracking error than the other patterns.

Figure 3.9 provides us with an interesting insight. As seen, the COM interpolator generally gives accurate readings at multiples of $45^{\circ}$ angles around the panel and tracking deviation occurs at the other angles. An angle-sweep of all the panels can help understand this phenomenon better. 


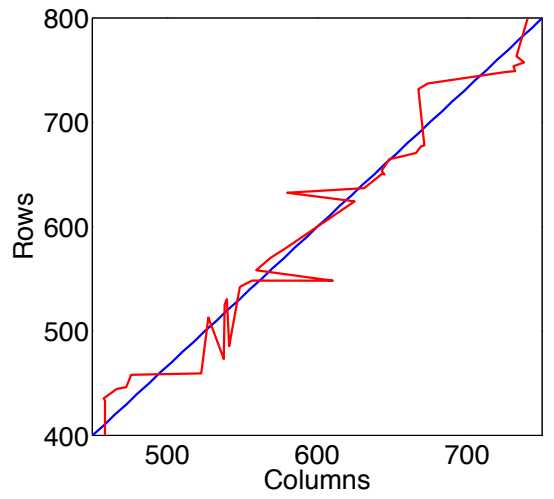

(a) Interleaved

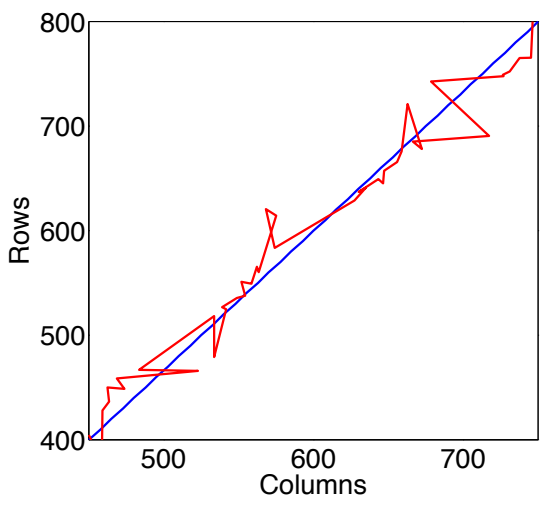

(c) Angled Cross

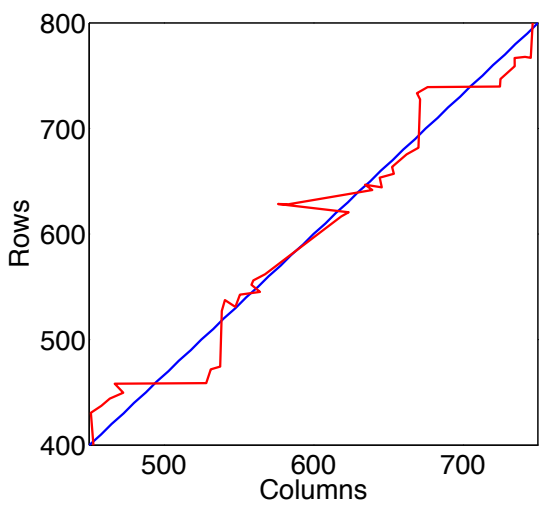

(e) Double T

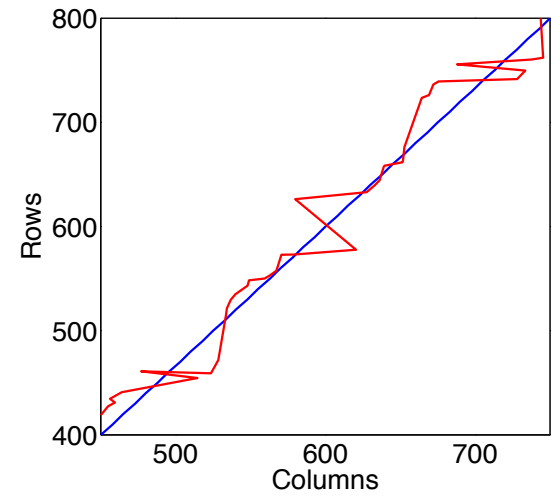

(b) Diamond

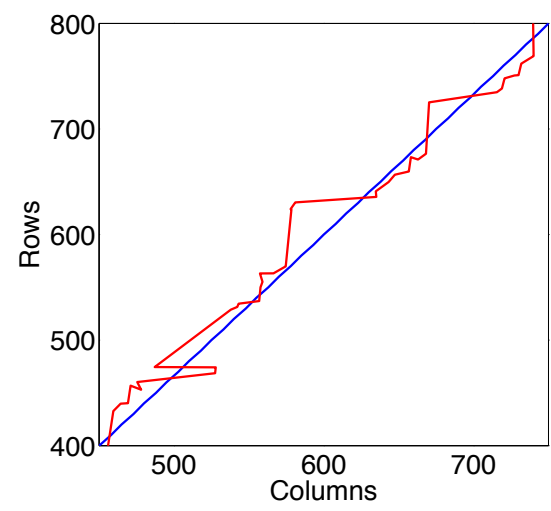

(d) Diamond-Interleaved

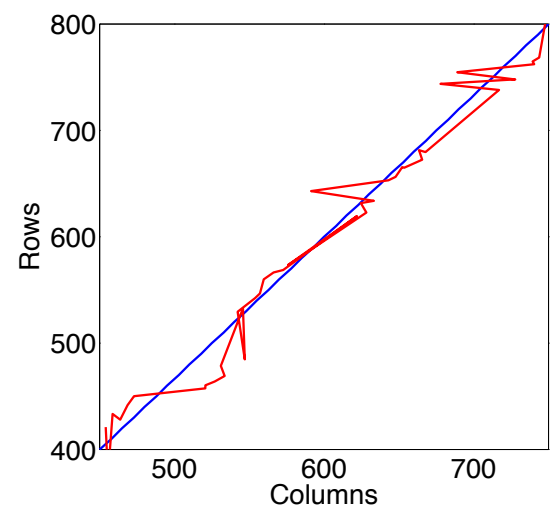

(f) Modified double T

Figure 3.8: A 7.5mm radius finger moved in a diagonal path with uniformly distributed random noise of 0.3 amplitude for different grid patterns. The units for rows and columns are in pixels. Blue: Exact Route, Red: Estimated Route. (100 pixels $=5 \mathrm{~mm}$ ). Figure is best viewed in color. 


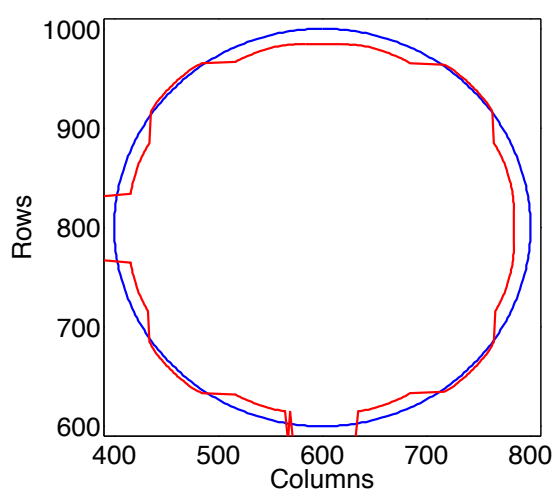

(a) Interleaved

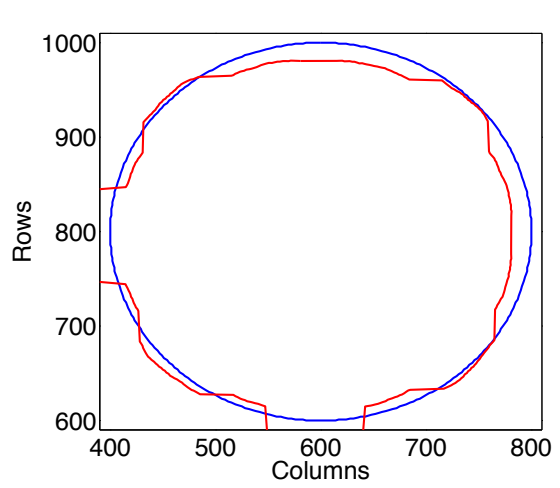

(c) Angled Cross

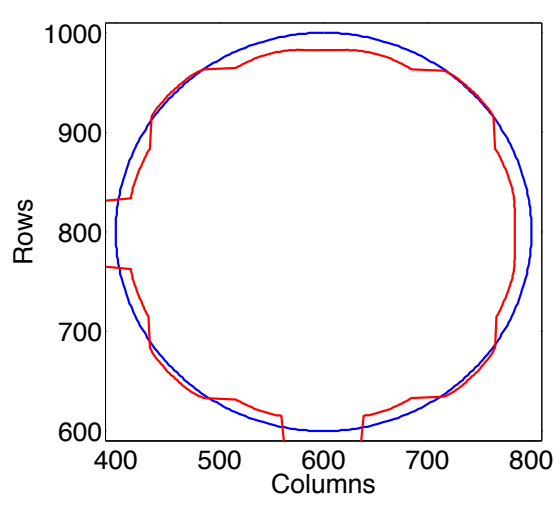

(e) Double T

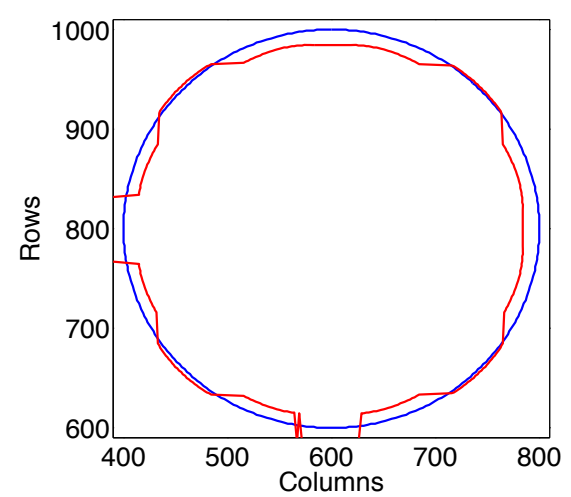

(b) Diamond

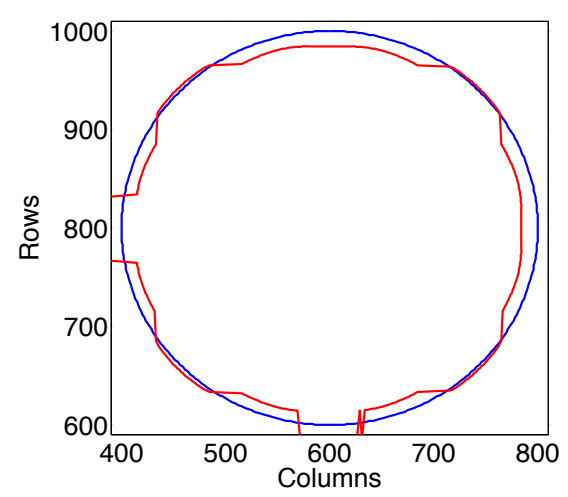

(d) Diamond-Interleaved

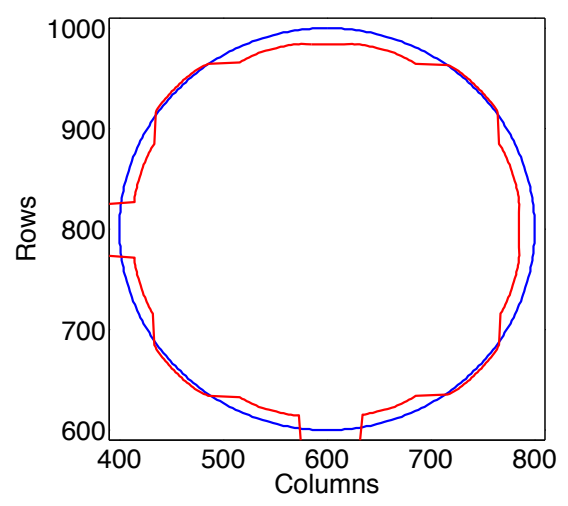

(f) Modified double T

Figure 3.9: A 7.5mm radius finger moved in a circular path for different grid patterns. The units for rows and columns are in pixels. Blue: Exact Route, Red: Estimated Route. (100 pixels $=5 \mathrm{~mm}$ ). Figure is best viewed in color. 
Table 3.2: Circular movement mean tracking error $(\mathrm{mm})$ with finger radius $7.5 \mathrm{~mm}$ and no noise.

\begin{tabular}{ccccccc}
\hline & Interleaved & Diamond & Angled Cross & Diamond-Interleaved & Double T & Modified double T \\
COM & $\mathbf{0 . 9 1 1 4}$ & 1.0037 & 1.0980 & 1.0028 & 1.0171 & 1.0011 \\
\hline
\end{tabular}

\subsubsection{Angle Sweep Movement}

An angle sweep per every $5^{\circ}$ was performed to check the performance of the grid patterns in terms of deviation from the exact (or true) route. The results are presented in Table 3.3 and Figure 3.10. The interleaved grid performance was better than the rest as shown in the table by bold font cell. The angled cross grid, on the other hand, reported the highest tracking error. As seen from Figure 3.10, the interpolator has a noticeable deviation for horizontal and vertical movements. Therefore, it can be stated that even with a less robust COM interpolator, interleaved pattern has the least tracking error and that COM suffers from high bias at multiples of $90^{\circ}$ angles.

Table 3.3: Angle sweep mean tracking error ( $\mathrm{mm})$ with finger radius $7.5 \mathrm{~mm}$ and no noise.

\begin{tabular}{ccccccc}
\hline & Interleaved & Diamond & Angled Cross & Diamond-Interleaved & Double T & Modified double T \\
COM & $\mathbf{0 . 8 2 6 2}$ & 0.9092 & 0.9788 & 0.9085 & 0.9193 & 0.9082 \\
\hline
\end{tabular}

Figure 3.11 shows the median, maximum and minimum tracking error along with mean error for circular and angle sweep movement. The conclusion still remains the same that the interleaved pattern has the overall least tracking error out of the six patterns.

The diamond pattern is widely used because it is very easy to manufacture and has a simple design. However, in this chapter, it has been shown that OEMs should consider adapting to new patterns so that higher accuracy can be achieved. Different companies like Synaptics and Avago Technologies use their own patented designs as explained earlier in the chapter. The main objective of this research is to compare performance of various patterns using a simulation environment. 


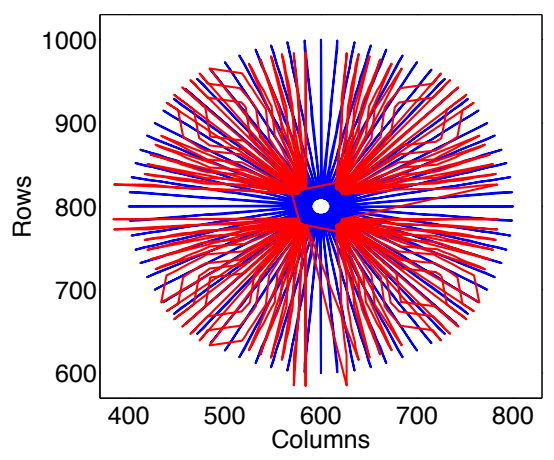

(a) Interleaved

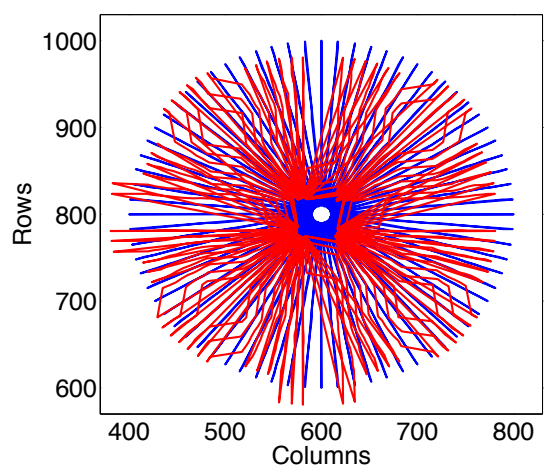

(c) Angled Cross

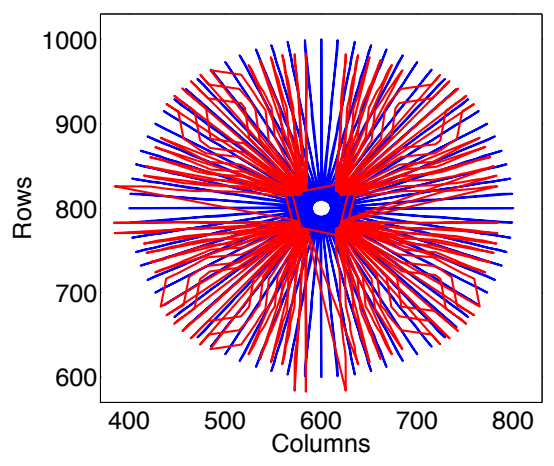

(e) Double T

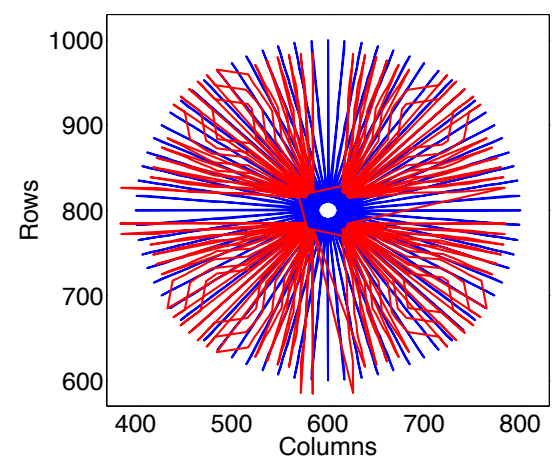

(b) Diamond

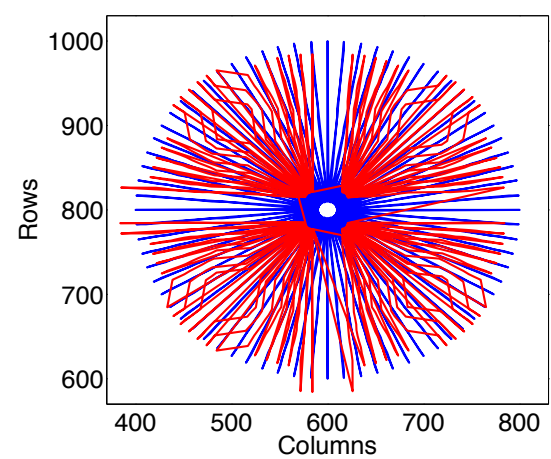

(d) Diamond-Interleaved

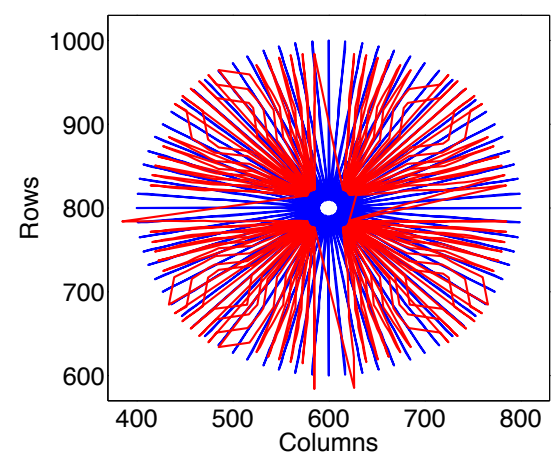

(f) Modified double T

Figure 3.10: Angle sweep performed by a $7.5 \mathrm{~mm}$ radius finger for different grid patterns. The units for rows and columns are in pixels. Blue: Exact Route, Red: Estimated Route. (100 pixels $=5 \mathrm{~mm}$ ). Figure is best viewed in color. 


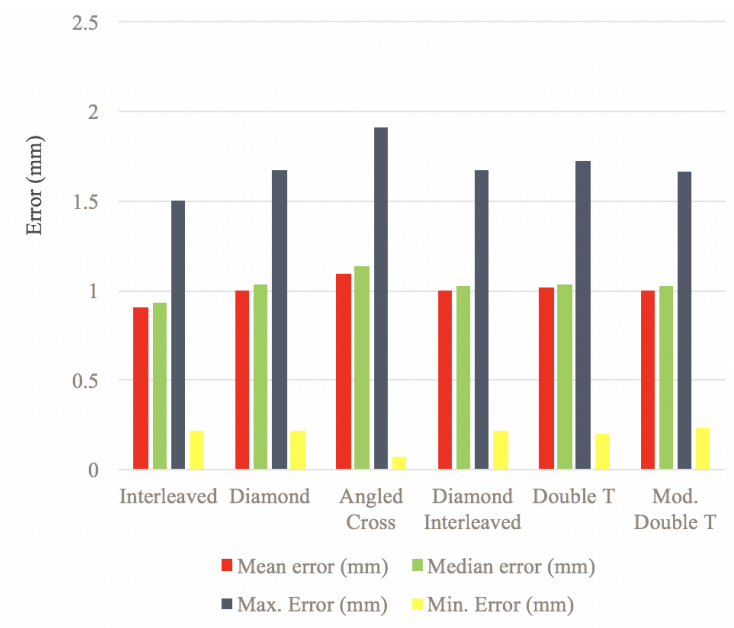

(a)

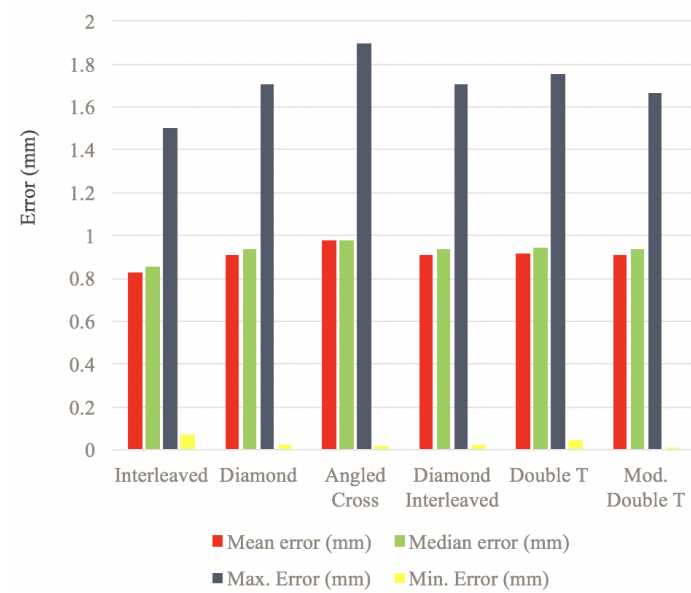

(b)

Figure 3.11: (a) Circular movement tracking error in mm with finger radius $7.5 \mathrm{~mm}$ and no noise (b) Angle Sweep movement with Finger radius $7.5 \mathrm{~mm}$ with zero noise [In Color]

The methodology proposed can work for both self and mutual capacitance systems. Earlier mutual capacitance circuits called for a separate pin out for every single row and column intersection. This has been changed with the advent of charge transfer technique which will be discussed in Chapter 4. Even then, the transformation from a small-scale design to a large-scale one is not a simple process. It involves study of cost and manufacturability, and a trade-off has to be established between feasibility and accuracy while designing sensor patterns. The first step in obtaining an optimal grid pattern which can be used for both small and large-scale touch panels, without extra cost and performance degradation is to define a methodology to test accuracy of existing grid patterns. In this chapter, such a methodology is devised for different grid pattern designs.

\subsubsection{Tracking Error Measurement Apparatus for Grid Pat- terns}

Capacitive touch controller manufacturers do not tend to release their research in the form of academic literature, therefore making it difficult to understand grid patterns, 
noise filtering and interpolation algorithms that are used in industry. However some companies do sell various kinds of capacitive touch demo boards for hobbyists and researchers [69, 70]. Microchip manufactures and sells a projected capacitive touch demo board equipped with a multi-touch controller and a main PIC32 processor shown in Figure 3.12(a).

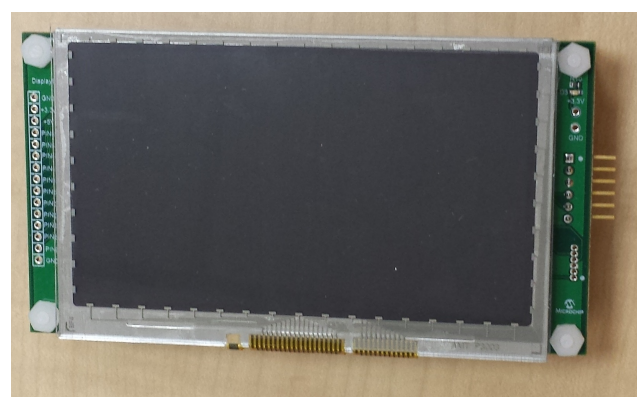

(a)

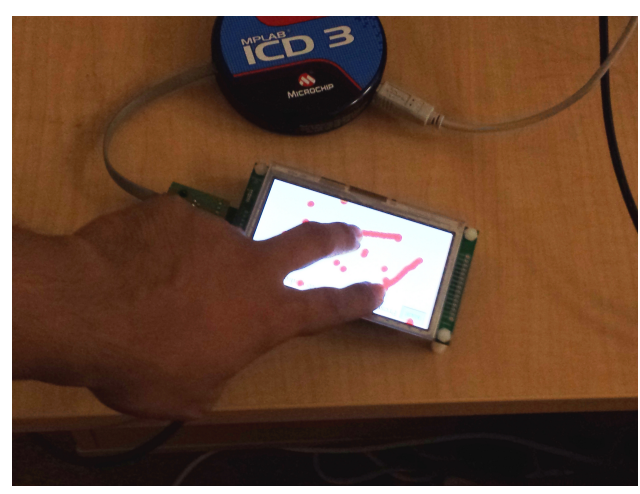

(b)

Figure 3.12: (a) Microchip projected capacitive touch board (top view) (b) MPLAB PCAP controller detecting track of two fingers, shown in red color.

The board is a stand-alone solution which has all the components embedded in a single PCB. It does not require a starter kit or a plug in module to run. The board uses a PIC32MX795F512L micro controller to control the LCD using LowCost Controllerless (LCC) graphics and a MTCH6301 micro controller to handle the capacitive sensing signals. Figure 3.12(b) shows the multi-touch capability of PCAP board.

As we did not have access to the raw touch data captured by the touch controller, we constructed a metallic finger and moved it on the screen so that we could calculate the tracking accuracy of the touch controller. We used XPS Newport motion stage [71] (Figure 3.13) and attached a metallic stylus to it. The idea was to fix the touch panel on the motion stage and then perform diagonal movement over the screen using the stylus. The motion stage was controlled using a user interface designed in Labview.

The touch controller used Inter-Integrated Circuit (I2C) protocol to transfer data to 


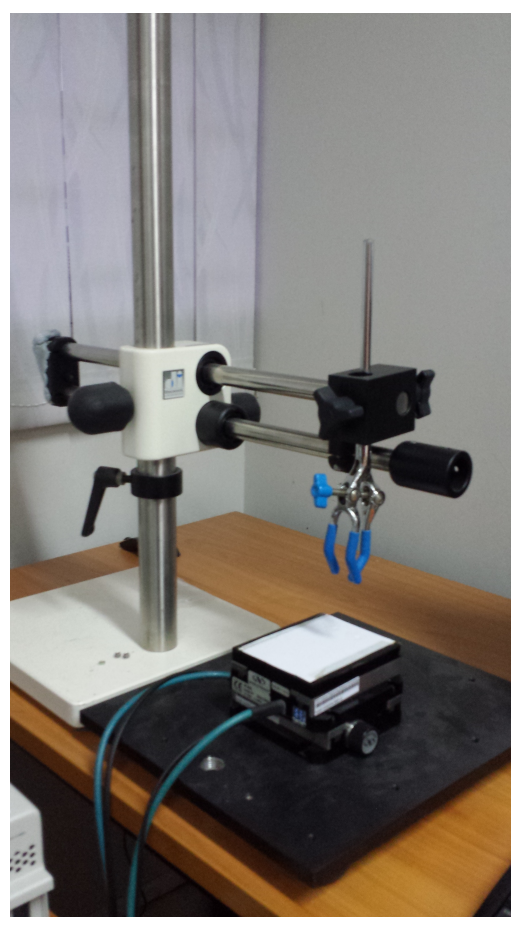

Figure 3.13: XPS NewPort motion stage controller.

microprocessor and the data was read out of the microprocessor using $\mathrm{C}$ language. The touch values captured by the controller were transformed into screen coordinates and graphic commands were sent to the LCD controller to draw points on those coordinates. As we knew our true coordinates from motion stage and estimated coordinates from the PIC controller, we used these values to determine the average mean linearity error which was approximately $2 \mathrm{~mm}$, which is huge for a typical capacitive touch screen. The results are shown in Figure 3.14. The whole experiment set-up can be seen in Figure 3.15.

The demo board used in this simulation had a $17 \times 13$ diamond pattern embedded in it. This is slightly different from the simulated case of 16 rows and 12 columns. This difference does not require any adjustment before comparison of results. The start and end point for diagonal movement is from $(4,4)$ to $(10,10)$ electrodes on the grid and thus the overall size of the panel is irrelevant.

Unfortunately, as we did not have access to other sensor designs, we could not test them under real conditions using this set-up. The tracking error will always be higher in real life for a grid pattern than in simulation environment. If we see the 


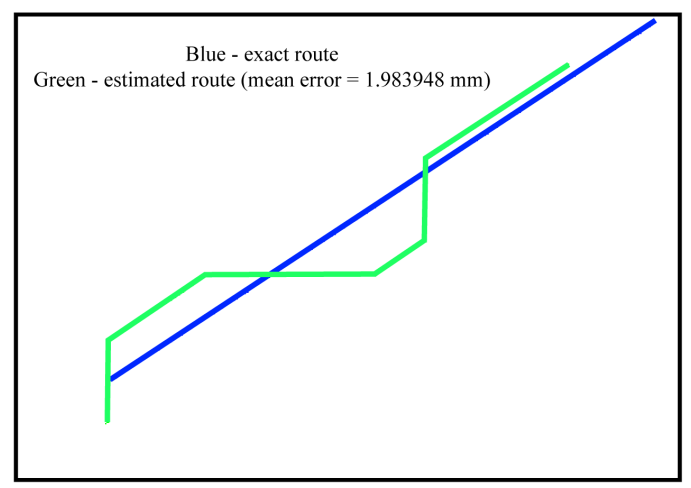

Figure 3.14: Robotic finger moving in a diagonal path. True path is shown in blue and estimated path is shown in green. Mean error is $2 \mathrm{~mm}$. Figure best viewed in color.

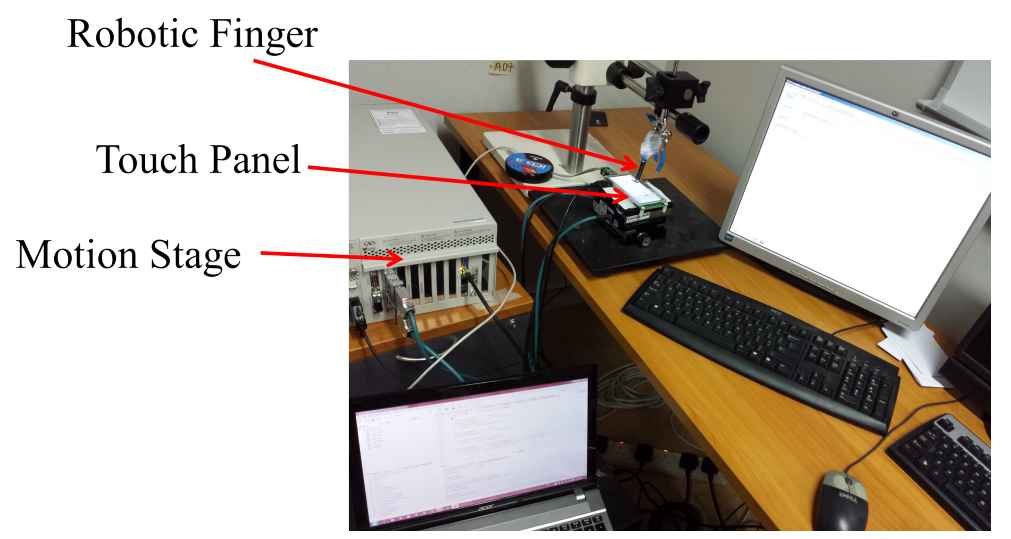

Figure 3.15: Capacitive touch panel hardware testing using motion stage.

results in Table 3.1, the mean tracking error for interleaved pattern in simulation is $0.6 \mathrm{~mm}$, whereas mean tracking error in real environment is $1.98 \mathrm{~mm}$. This is due to noise induced by LCD underneath and the random noise by the charger. The margin difference in this particular comparison is of $1.38 \mathrm{~mm}$.

\subsection{Designing Hybrid Patterns using Fourier Trans- form}

As explained in Section 3.1.2, there are numerous grid patterns proposed by designers over the years. It is not feasible for us to design, fabricate and test these grid 
patterns in hardware because of the lack of resources. A simulation environment, on the other hand provides a viable option to construct a methodology for these grid patterns as it is easy to simulate a complex design and environmental variables can be controlled for testing under different conditions.

From image and signal processing literature, it is known that Fourier phase usually contains more information about an image than Fourier magnitude [72]. As we are constructing sensor patterns as binary images, one way of designing hybrid patterns would be to combine the Fourier phase from one pattern (binary image) with Fourier magnitude of the other pattern (binary image). As an example, if we try to combine Fourier phase of Interleaved pattern (least tracking error) with Fourier magnitude of Angled Cross pattern (highest tracking error) in Figure 3.16, hypothetically, one should get a hybrid pattern with performance close to interleaved pattern (as phase of an image contains most information about an image).

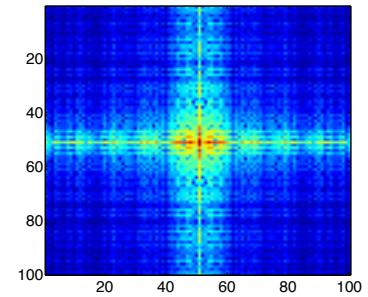

(a) Interleaved Magnitude

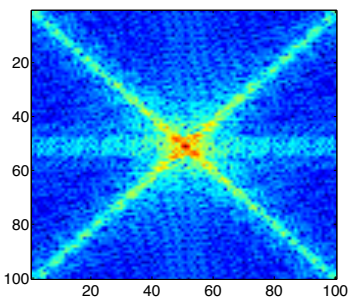

(c) Angled Cross Magnitude

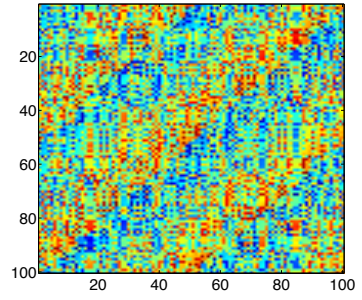

(b) Interleaved Phase

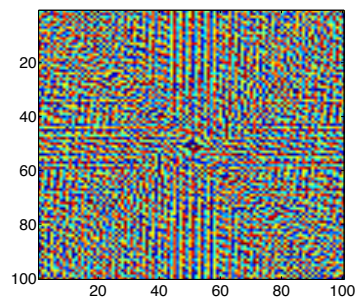

(d) Angled Cross Phase

Figure 3.16: Fourier log magnitude and phase for interleaved and angled cross. It can be seen from the magnitude plot that angled cross has high frequency values at multiples of 45 degrees whereas the interleaved pattern has high frequency values at multiples of 90 degrees (best viewed in color).

The hypothesis that hybrid pattern np. 1 will perform much better than hybrid 


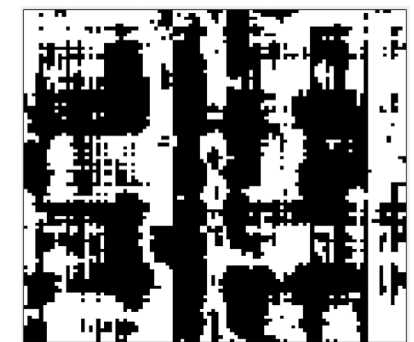

(a)

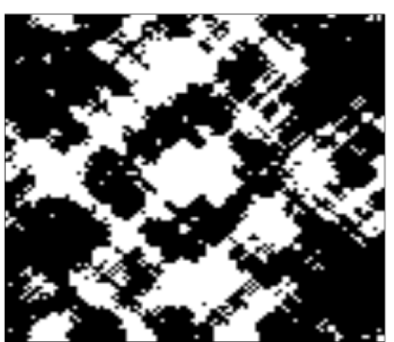

(b)

Figure 3.17: (a) Hybrid pattern no. 1 consisting of Fourier Magnitude of Angled Cross and Phase of Interleaved Pattern, (b) Hybrid pattern no. 2 consisting of Fourier Magnitude of Interleaved and Phase of Angled Cross Pattern.

pattern no. 2 does not hold for this particular case. As seen below in table 3.4, the performance difference is negligible.

The reason for this is that the original grid pattern basic elements are simple binary images which result in even simpler binary hybrid images and there is not much unique information available in both hybrid patterns to have a meaningful impact on the performance. As it can be seen from Figure 3.17 that the patterns do not look much alike interleaved or angled cross grid pattern.

Thus, we can conclude from this experiment that although we can test various hybrid patterns by combining magnitude and phase of various patterns together, more investigation needs to be done in the future in order to understand how to optimize the hybrid patterns using linearity or non-linearity of Fourier phase. Theoretically, the magnitude of the Fourier transform will have negligible impact on the performance of the grid pattern provided that the pattern is complex enough to be reconstructed by the phase alone.

Table 3.4: Performance comparison of hybrid patterns with angled cross and interleaved pattern.

\begin{tabular}{ccccc}
\hline \multicolumn{5}{c}{ Angle Sweep } \\
\hline & Angled Cross Grid & Interleaved Grid & Hybrid Pattern No. 1 & Hybrid Pattern No. 2 \\
COM & Mean Error (mm) & Mean Error (mm) & Mean Error (mm) & Mean Error (mm) \\
\hline
\end{tabular}




\subsection{Designing Efficient Patterns by doing Geo- metric Changes}

So far, we have measured tracking accuracy of a few existing sensor patterns using a simulation environment. In this section, we want to look into how geometry of a single grid element affects its efficiency. The term efficiency is very broad as it can cover accuracy, precision or even Signal to Noise Ratio (SNR). In this very straight forward and simple methodology, efficiency can be referred to as:

$$
\epsilon=\frac{\text { Sensing area }}{\text { Total device area }}
$$

The basic idea was taken from [73], where geometric equations were used to find out the efficiency of interdigitized array electrode sensors. It can be seen from (3.2) that the maximum possible efficiency would equal 1 if total area of the sensor pattern equals its total sensing area. Of course, that is not physically possible, but the objective is to redesign sensors in such a way that the sensing area is increased and the efficiency gets as close to 1 as possible. In this section, interleaved pattern is used as the test subject because (a) it is the most accurate pattern according to our methodology and (b) it displays the effectiveness of this method nicely. Let us apply (3.2) to our interleaved pattern basic element shown in Figure 3.18. The sensing area for an unoptimized design is shown in red, whereas X and Y electrodes are shown in blue and red colors respectively. The space covered in white inside the sensor boundaries is the unoptimized sensing area. For the sake of simplicity, the design is kept symmetrical and it is assumed that $G$ and $E$ remain constant throughout the sensor area.

Equation (3.3) shows the efficiency for an unoptimized interleaved sensor. The underlined term $20 G^{2}$ is important here which is the representative of the unoptimized 


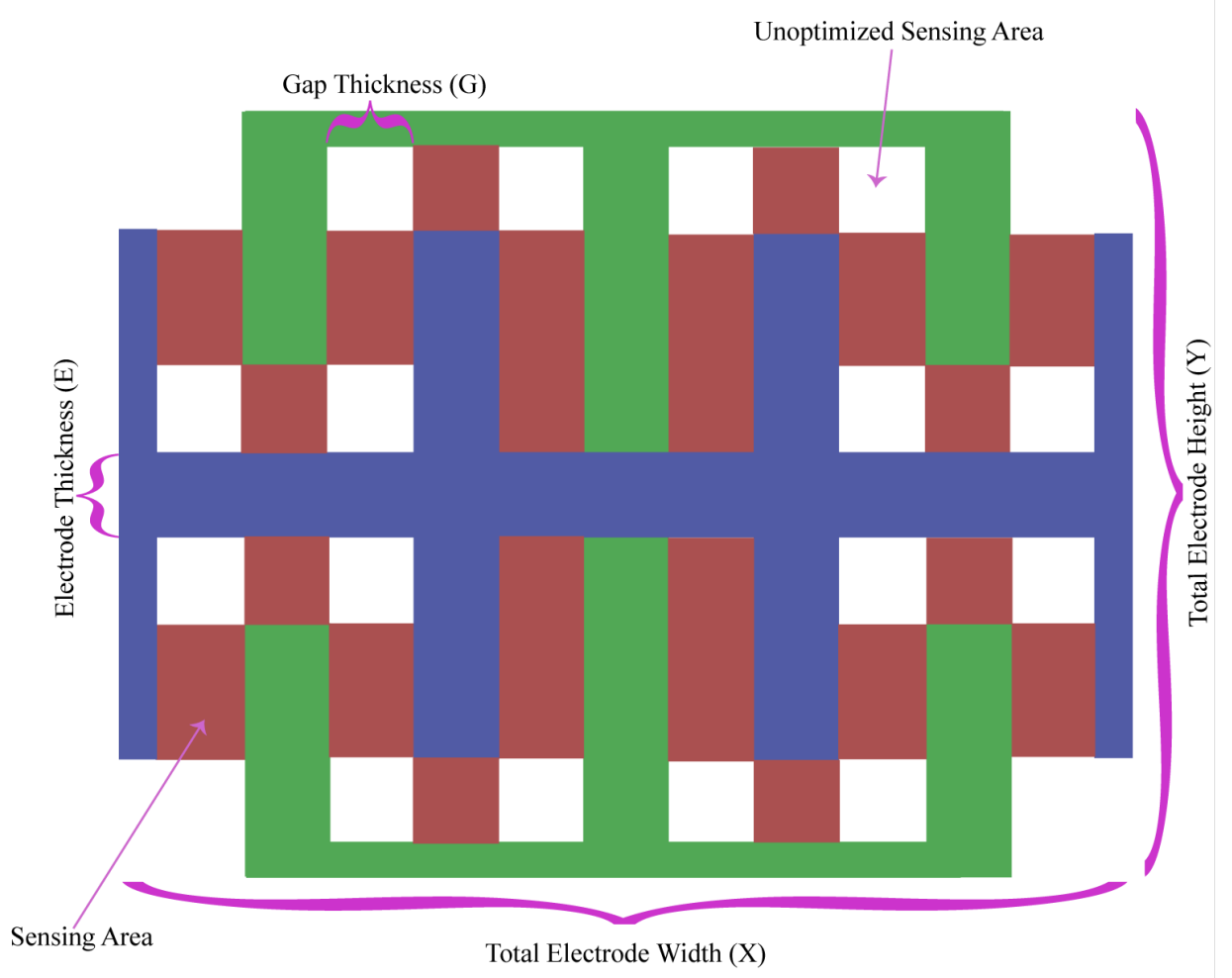

Figure 3.18: Interleaved sensor with unoptimized gap regions not included as part of sensing area (best viewed in color).

sensing area that we wish to reduce.

$$
\epsilon=\frac{X Y-2 E X-6 E Y+7 E G+E^{2}-\underline{20 G^{2}}}{X Y} .
$$

By looking at Figure 3.18, one realizes that some of the white area can be transformed to red if the sensor is redesigned in such a way that the contact area between $\mathrm{X}$ and $\mathrm{Y}$ electrodes at corners is increased. It can be done by simply rounding the corners in the sensors as shown in Figure 3.19. The efficiency of rounded corner sensor may be calculated using (3.4).

$$
\epsilon=\frac{X Y-2 E X-6 E Y+7 E G+E^{2}-\underline{4 G^{2}}}{X Y} .
$$

Note that this equation is identical to (3.3) except that the underlined $20 G^{2}$ term is now changed to $4 G^{2}$. This difference can be seen from the figures as well. Because 


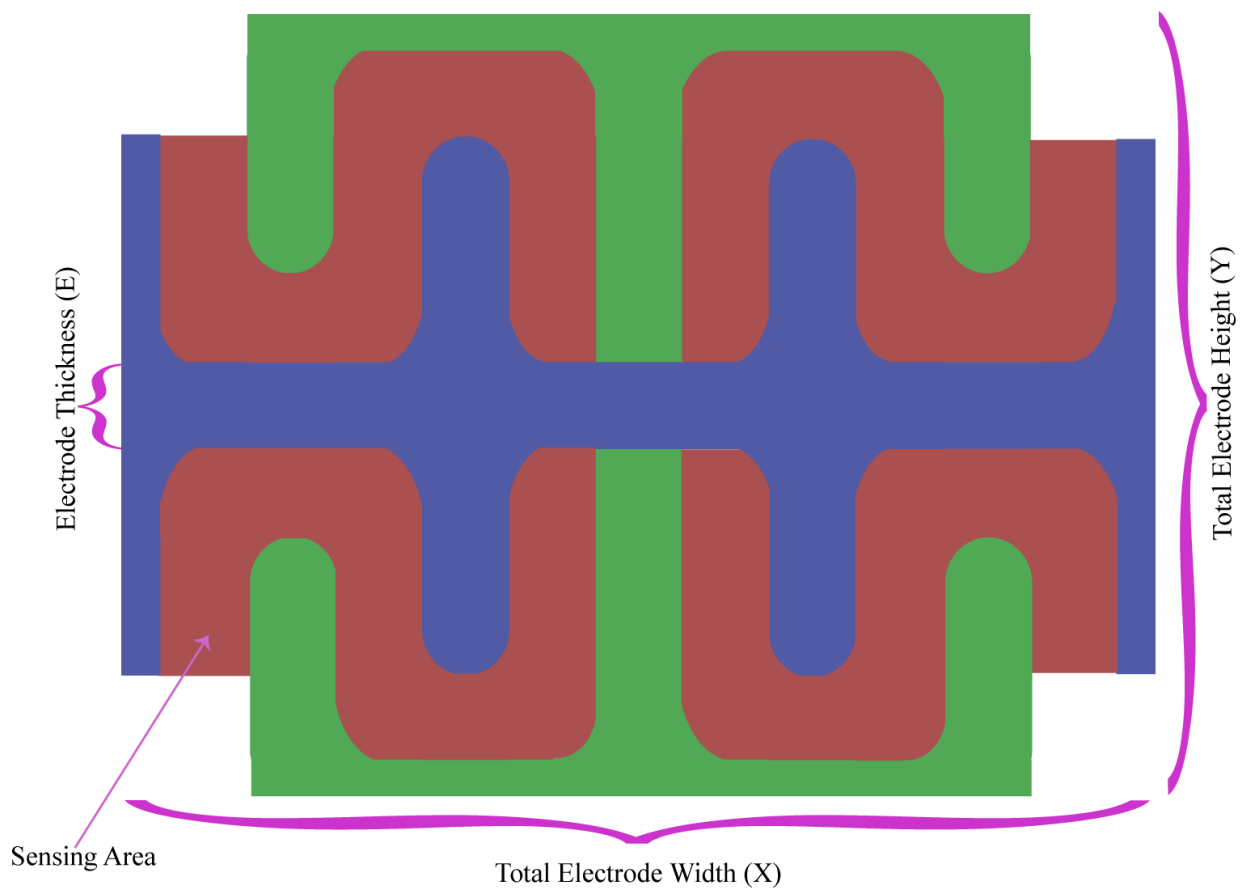

Figure 3.19: Redesigned interleaved sensor with curved corners to increase the efficiency of the sensor (best viewed in color).

the said term is subtracted from the numerator, the logical method to improve the efficiency would be to minimize this term as much as possible and this is exactly what is being achieved here. Rounding the $90^{\circ}$ corners of the traditional interleaved sensor reduces the last term in numerator from 20 to 4 and thus increases the efficiency by a sizable margin. It can be stated that by doing such simple manufacturing changes, one can significantly improve sensitivity of grid patterns.

\subsection{Measuring Sensitivity of Capacitive Pattern using ANSYS Capacitance Solver}

So far we have only tested the tracking accuracy of sensor patterns and concluded that interleaved pattern is more suitable for capacitive touch panels due to its low tracking error. These results were published as an article [27] and as proceedings [28] and were one of the first pieces of academic literature in this field. Recently, 
new research has surfaced which talks about electrostatic simulation methodology for capacitive touch panels [74, 75]. In [74], touch sensitivity for various patterns was analyzed using simulations. The software used was COMSOL [76], which uses Finite Element Method (FEM) for calculating the capacitance matrix. Detail of FEM is not a focus of this thesis but briefly stating, FEM tries to approximate the result of partial differential equations (PDEs) by solving numerical model equations which are in turn constructed using different types of discretizations. One can design sensor patterns and a conductive finger in COMSOL and run FEM to calculate the change in capacitance by the introduction of finger to the panel. A strong correlation was discovered between high sensitivity and location of fringing fields on a pattern [74]. Fringing fields form the sensing area of a touch panel so they are directly proportional to the sensitivity of a sensor pattern. Similar simulations were performed in [75] using both COMSOL and ANSYS Q3D Method Of Moments (MOM) solvers. The difference between MOM and FEM is that MOM uses integration instead of differentiation for the equation to be solved. MOM has overtaken FEM in recent years in terms of usage because of fast integration solvers such as FFT-based ones etc.

Using the ANSYS capacitance solver, one can find the capacitance value of the touch sensors with and without finger present on the panel and then calculate the percentage decrease in the capacitance due to finger presence. Here, simulations were performed using ANSYS Q3D solver (v. 16.0) to check how geometry of patterns affect their touch sensitivity. Note that sensitivity is different from tracking accuracy. A more sensitive pattern is not necessarily less prone to tracking errors. Figure 3.20 shows the interleaved pattern while a metallic copper cylinder representing a finger is resting on it. The finger radius is $7.5 \mathrm{~mm}$ so that it covers more than one electrode in all directions. The finger's center is aligned with the center of a sensor right beneath the finger and is not changed throughout the simulation. The change in capacitance $\Delta C$ due to finger presence is recorded and is shown in Figure 3.21. Similarly, simulation results for diamond, angled cross, diamond-interleaved and double $\mathrm{T}$ are presented in Figure 3.21. The modified double T pattern has been left 


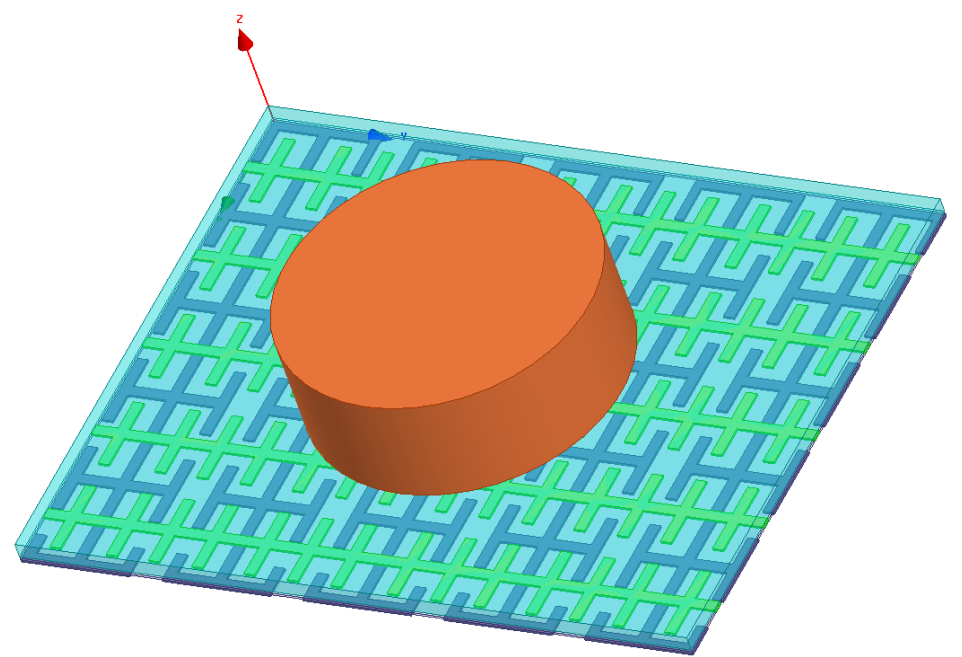

(a)

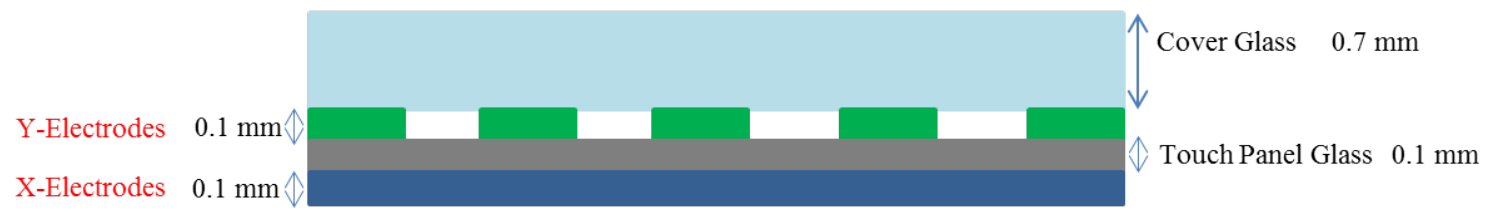

(b)

Figure 3.20: (a) Interleaved pattern with finger as designed in ANSYS Q3D solver (b) Side section showing the widths of various layers in the simulation stack (figure not drawn to scale and is best viewed in color).

out because of its high similarity with double T pattern. Based on the results, it can be stated that interleaved pattern has higher touch sensitivity than the rest of the patterns.

There are some interesting conclusions that can be drawn from this test:

1. Angled cross pattern has higher sensitivity than diamond pattern despite having poor tracking performance. The reason for this anomaly is that sensing and driving electrodes in angled cross pattern have more sensitive regions where the edges of both electrodes meet as compared to diamond pattern. This is highlighted by red lines in Figure 3.22. The angled cross pattern is designed in a way to maximize the contact area between $\mathrm{X}$ and $\mathrm{Y}$ electrodes, something that is not fully utilized in diamond pattern. As demonstrated in [74], there is 


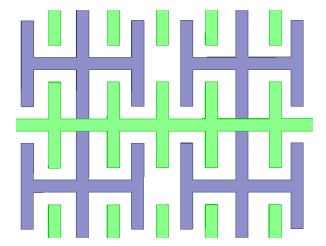

(a) Interleaved

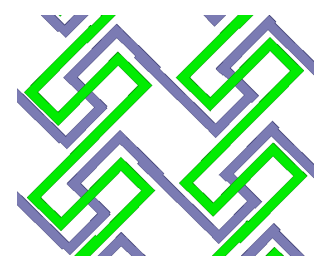

(e) Angled Cross

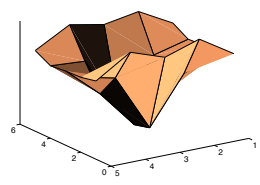

(b) $\Delta C=37 \%$

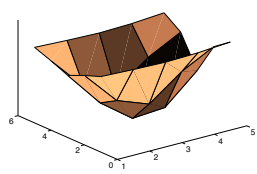

(f) $\Delta C=33 \%$

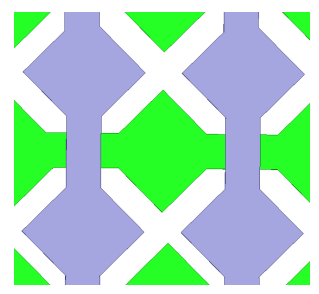

(c) Diamond

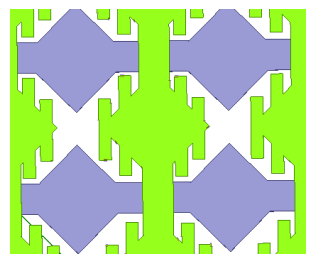

(g)

Diamond-

Interleaved

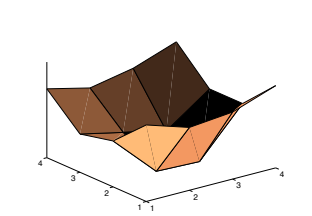

(j) $\Delta C=27 \%$

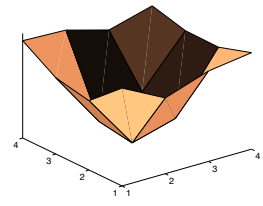

(d) $\Delta C=17 \%$

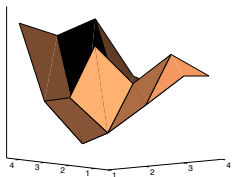

(h) $\Delta C=18 \%$

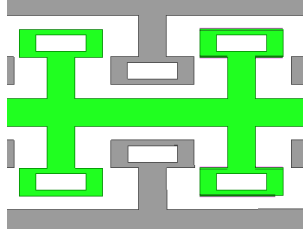

(i) double $\mathrm{T}$

Figure 3.21: Simulation results for sensor patterns change in capacitance with 3D plots.

a strong correlation between the high touch sensitivity areas and location of fringing fields. The fringing fields of angled cross pattern can be enhanced by rounding the corners as was done with interleaved pattern in Section 3.4. An extension of the current experiment can be proposed for future where sensitivity is measured at multiple locations across the panel and tracking error can be estimated from difference in results. However, here we are only interested in capacitance difference when finger is at a stationary position.

2. Designing a more sensitive pattern involves improving the fringing field locations at the intersection of sensing and driving lines.

3. Designing a more accurate pattern involves ensuring that finger has a smooth transition from one sensor to another. Diamond pattern when compared to angled cross has a smoother design between sensors when compared with angled 


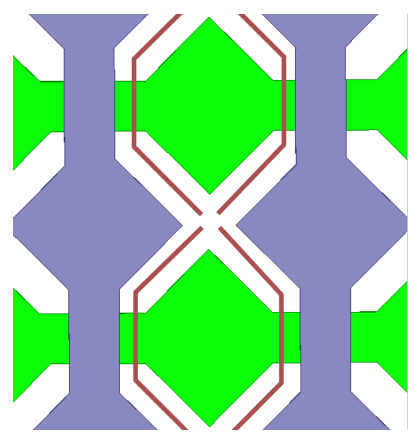

(a)

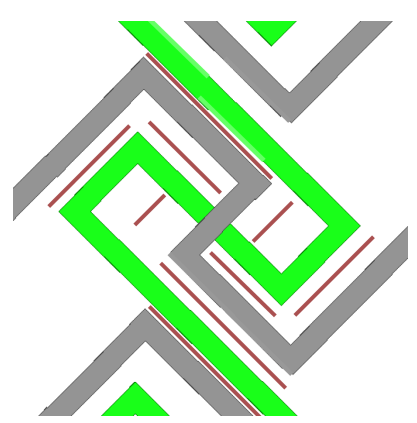

(b)

Figure 3.22: Location of fringing fields (a) Diamond (b) Angled Cross pattern (best viewed in color).

cross pattern hence its tracking error is lower.

\subsection{Validation of Proposed Methodology}

It is shown using literature review, that there are a number of patented patterns but no single method to fairly compare and evaluate them. This motivated us to explore possible evaluation solutions. Therefore, in this Chapter, we propose a methodology for sensor patterns by testing and comparing six designs using a simulation environment. We placed a 2-D Gaussian shaped finger on a touch panel designed like a binary image and performed various movements including diagonal, circular and angle sweep to calculate mean tracking error using COM interpolator. We compared and contrasted the results of patterns amongst each other. It was found that the interleaved pattern performed better than the rest. The methodology 
presented can be extended to any sensor pattern design.

Due to the lack of literature and ground truth data, we were aware of the question from the very beginning whether our proposed algorithm is fair or valid. Below, we write down some measures taken to validate the proposed techniques.

First, intensive communications with touch panel industry (Solomon Systech Limited and Sharp Corporation) was carried out before we dived into research to ensure that our problem statement is indeed valid and meaningful. The industry wished to have an answer to the problem statement we proposed and due to multiple reasons, they did not have a solution.

Second, when we started to simulate the patterns, we chose valid basic elements of a grid pattern from published patents. Samples were taken from patents published by touch panel manufacturers, for example $[52,53]$. When we simulate fingers, the finger model was designed to be a 2D Gaussian surface based on already published literature in this research field $[58,65,66,67]$. This design was also validated based on our personal communications with touch panel industry during the course of this research, where it was observed that the set of measurements reported by the touch controller in a typical testing environment appears substantially Gaussian-shaped.

Third, the methodology was designed initially in Matlab and was tested with various finger movements along $\mathrm{x}$ and $\mathrm{y}$ axis on the panel with and without addition of noise. Later on, we also adopted ANSYS MOM solver to provide a methodology for testing sensitivity of grid patterns and it was concluded that sensitivity and tracking accuracy depend on different aspects of the sensor design.

Forth, we are aware that pure simulation may have limitations to reflect the practical problem at hand, we performed the diagonal movement experiment in hardware, using a robotic finger (capacitive stylus) and a XPS NewPort motion stage controller. The mean tracking error was found out to be around $2 \mathrm{~mm}$. Fourier transform (magnitude and phase) of patterns was studied and a hybrid pattern was proposed combining Fourier magnitude and phase from two different patterns. Being able to treat capacitive touch sensor patterns as images helped us in designing hybrid 
patterns. Doing simple geometric changes to an existing pattern also helped increase the sensitivity of the pattern.

Finally, our simulation results were published as a journal article [27] and in IEEE proceedings [28] and were one of the first pieces of academic literature in this novel field. Recent publications in the same field have cited our work [77, 78, 79] thus proving that this work is advancing human knowledge in this particular field. The conclusions obtained by our sensitivity study are consistent with recent published academic literature studying the role of fringing fields in capacitive touch sensitivity [74].

\subsection{Conclusion and Future Work}

To provide a universal methodology for comparing the performance of different capacitive touch screen grid patterns was the main objective of this chapter. The tracking accuracy of six different grid designs was measured using a capacitive touch panel simulation environment. A methodology for testing tracking performance was designed in Matlab whereas ANSYS was used to test sensitivity of touch panels. Utilizing the methodology presented in this chapter, one can choose a suitable grid structure for their applications.

After looking at the results of the tracking error for diagonal, circular and anglesweep movements, it can be said that the interleaved pattern has the lowest tracking error in mm. The sensitivity of the interleaved pattern is also higher than the rest of the patterns under test.

According to the findings in this chapter, two conclusions can be drawn regarding the rationale for the choice of elemental grid structure:

1. Fringing field locations at the intersection of sensing and driving lines contribute to the sensitivity of a touch panel. 
2. Smooth transition of finger capacitance from one grid basic element to another ensures a more accurate touch panel.

How Fourier transform and non-linearity of phase can be used to construct an optimal pattern will be investigated further in the future. The results shown are based on the assumption that both finger and noise model is Gaussian. A more realistic finger and noise model, along with hardware testing using a robotic finger will be applied in the future to confirm our results and to find out other factors that govern the performance of sensor patterns for capacitive touch screens. 


\section{Chapter 4}

\section{Efficient Sampling and Recovery}

\subsection{Introduction and Related Work}

In the last chapter, sensor pattern design was discussed and a methodology was developed for testing sensor patterns. Hybrid patterns and geometric techniques to increase sensitivity of a touch sensor were also proposed. The second important part of a capacitive touch system is the sampling. As the touch panel size increases, it becomes evident that no matter how sensitive or accurate a touch sensor design is, chances are that without efficient sampling, user experience will not be great, as latency will occur constantly. In this chapter, structured and random sampling matrices are used in an attempt to propose efficient touch location recovery algorithm for capacitive touch panels.

As explained in Chapter 1, capacitive sensor electrodes are arranged on a touch panel in a grid formation, with each electrode representing a touch coordinate. The optimal spacing between electrodes is based on the diameter of a human finger, and is roughly $5 \mathrm{~mm}$. The disadvantage of adding more sensors in the panel in order to increase the resolution is that it results in an increase in scanning time. It is important that efficient scanning algorithms are developed to ensure the highest level of responsiveness and resolution regardless of the size of the touch panel. 


\subsubsection{Traditional Sampling Techniques}

Figure 4.1 shows the simplified block diagram of a capacitive touch panel. As already mentioned in Chapter 1, the touch panel consists of physical sensors, a sampling circuit and a post processing unit. In Figure 4.1, the driving lines are represented by $\mathbf{X}_{1} \ldots \mathbf{X}_{N}$, whereas the sensing lines are shown as $\mathbf{Y}_{1} \ldots \mathbf{Y}_{N}$. The sensor network shown here can be any one of the many sensor pattern designs discussed in Chapter 3. The capacitance at the intersections is represented by $\mathbf{C}_{1,1} \ldots \mathbf{C}_{N, M}$. The excitation driver sends out signals to the driving lines and the difference in charge $Q$ is sensed through the sensing lines. The host micro-controller is connected to the sampling circuit and processes the digital data in order to get the touch position. The processing at the host controller includes back-end noise filtering and subpixel interpolation. Although it can vary, the sampling circuit typically consists of the excitation driver, timing controller, analog to digital converter (ADC) and the analog front-end $[80,81,82]$. Similar to the sensor pattern design, different touch panel manufacturers utilize different sampling techniques for measuring capacitance. This chapter starts by explaining some of those sampling methods in detail below.

Sections 4.1.1.1 to 4.1.1.3 deal with electronics of some of the sampling methods used in industry. Readers who are only interested in the mathematical basis of the sampling methods proposed in this chapter, can skip to section 4.1.1.4 directly.

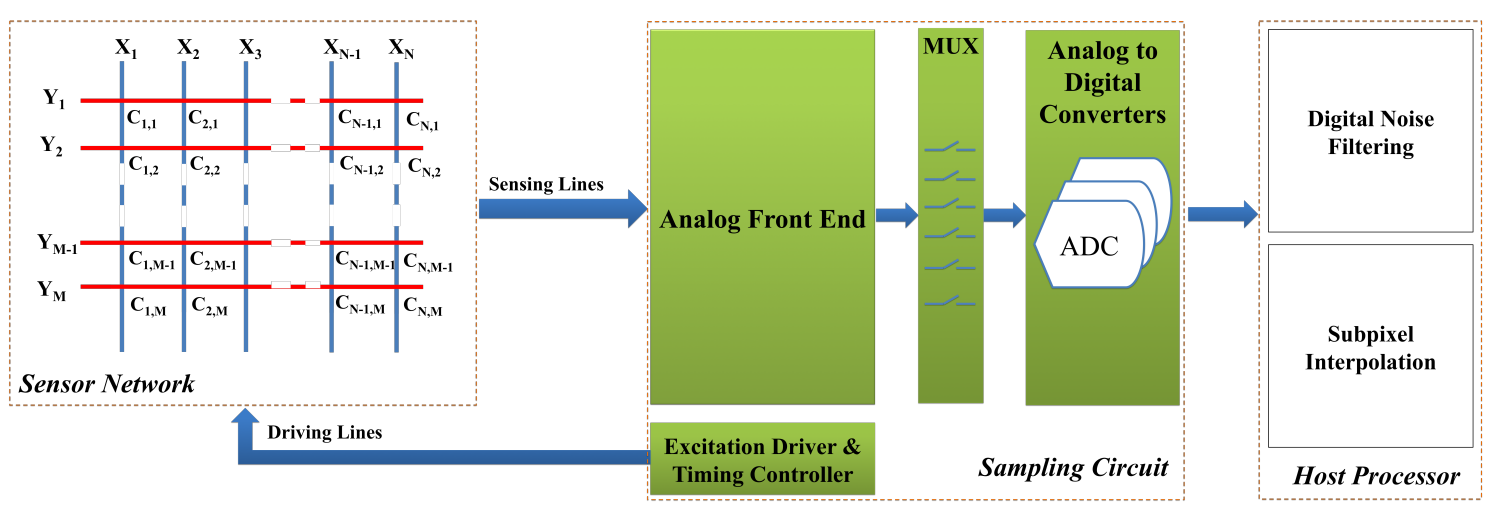

Figure 4.1: Simplified block diagram of a traditional capacitive touch panel system. 


\subsubsection{Relaxation Oscillator}

Figure 4.2 shows a simple relaxation oscillator topology to be used as the sampling circuit for capacitive touch panels. Initially the discharge switch $(\mathrm{S})$ is opened and the sensor capacitor $(\mathrm{C})$ is charged through the pull up resistor. The capacitor is charged until the voltage at the positive end of the comparator exceeds the threshold, changing the output from low to high. This transition will cause the switch to close and the capacitor quickly discharges causing the output of comparator to transition from high to low and the cycle repeats itself. The value at the output of flip flop $\left(\mathrm{f}_{\text {out }}\right)$ is representative of the capacitive sensor value [8].

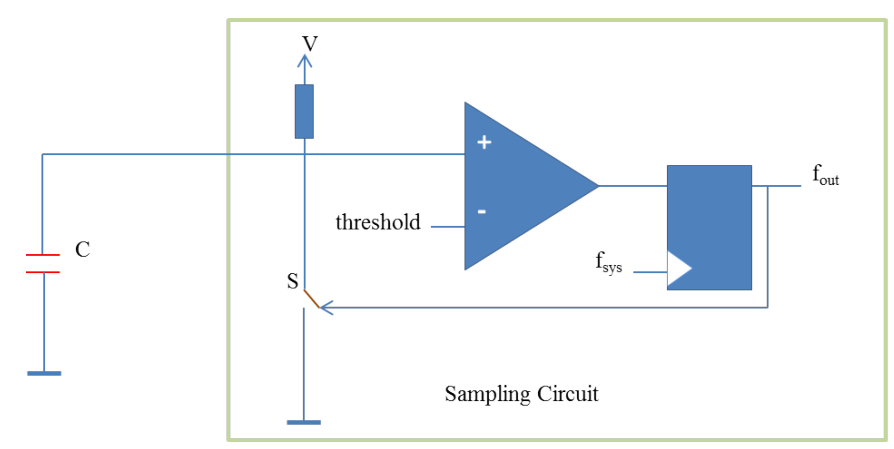

Figure 4.2: Simple relaxation oscillator topology. Figure reproduced from [8].

For a capacitive touch panel, each sensor is connected to a comparator and a flip flop. Based on the sampling rate, the excitation driver charges and discharges the capacitor continuously when the panel is in operation. If a finger presence is detected, the value of the $\mathrm{f}_{\text {out }}$ changes which is picked up by the host controller as a valid touch location.

\subsubsection{Sigma Delta Modulation}

Sigma Delta modulation is a popular technique with companies like Cypress and Microchip both having propriety patents on small modifications of the parent method. The traditional technique uses a switched capacitor network to detect touch. In Figure 4.3, the sensor capacitor $C$, an external capacitor $C_{\text {ext }}$ and a resistor $R$ is connected to the positive input of a comparator with its negative input connected 
to a threshold voltage value just like in relaxation oscillator method. Using small charge up and down steps, the voltage across external capacitor is regulated and that causes the comparator output to change in regular cycles. When a touch occurs, some of the charge at sensor capacitor is taken away by the finger and that causes the time period of the comparator output to change slightly. This change is detected by the host micro-controller as a valid touch input.

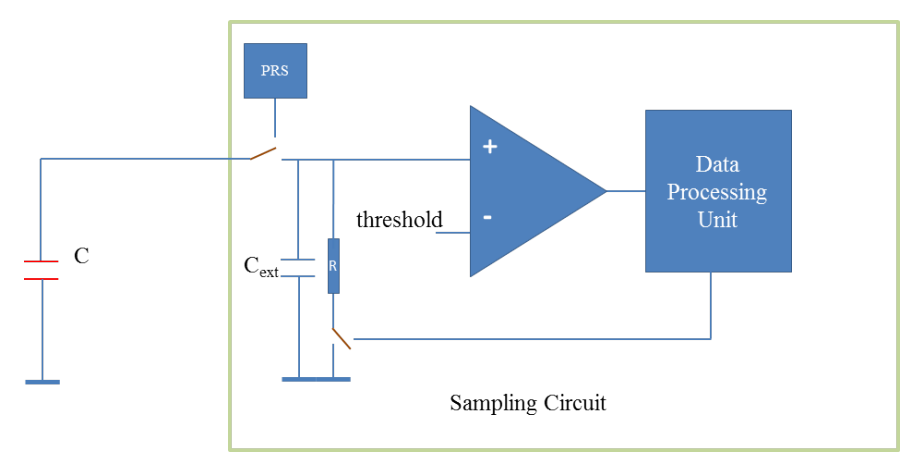

Figure 4.3: Simple sigma delta modulator topology. The Pseudo Random Sequence (PRS) generator is responsible for controlling the switch between external and sensor capacitor. Figure reproduced from [9] with simplification.

In Figure 4.3, the data processing unit consists of an ADC, oscillator and a timer along with a micro-controller. The bit stream resulting from the charge discharge cycle is ANDed with the ADC output to enable a timer. This timer output is used by the micro-controller to determine the level of capacitance change.

\subsubsection{Voltage Divider}

In a voltage divider configuration, a sensor capacitor and an internal capacitor are charged to opposite voltage twice. The first time this operation is performed is titled configuration 1. The second time the configuration is reversed and is titled configuration 2 .

Configuration 1:

Internal capacitor $\left(C_{i n t}\right)$ is discharged to $V_{s s}$ and sensor capacitor $(C)$ is charged to $V_{d d}$ 
Configuration 2:

Internal capacitor $\left(C_{i n t}\right)$ is charged to $V_{d d}$ and sensor capacitor $(C)$ is discharged to $V_{s s}$

After pre-charging, both capacitors are connected in parallel and are given ample time for their charges to settle. The time taken for the charges to reach a settling point is noted. As a finger comes closer to the sensor capacitor, the settling time increases by some margin and that signals the presence of a finger to the controller. A waveform representation of the voltage divider operation is shown in Figure 4.4 for finger and without finger.

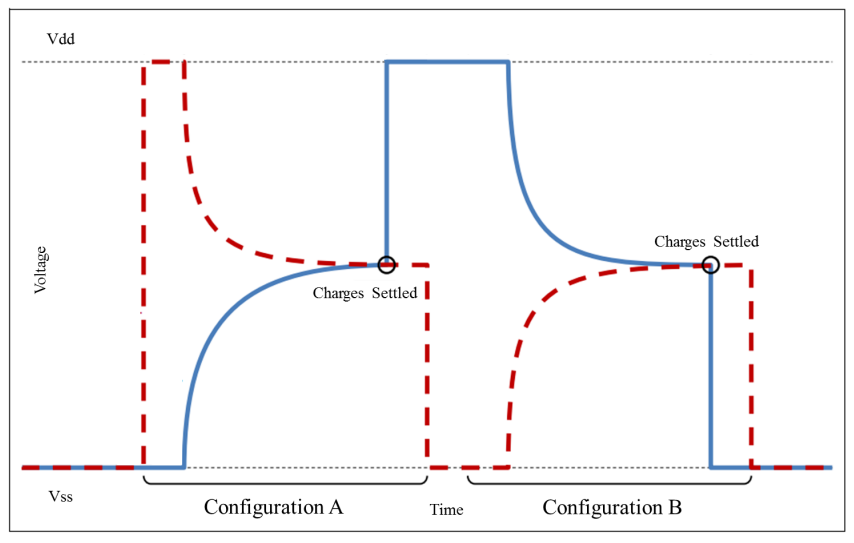

(a) Without finger

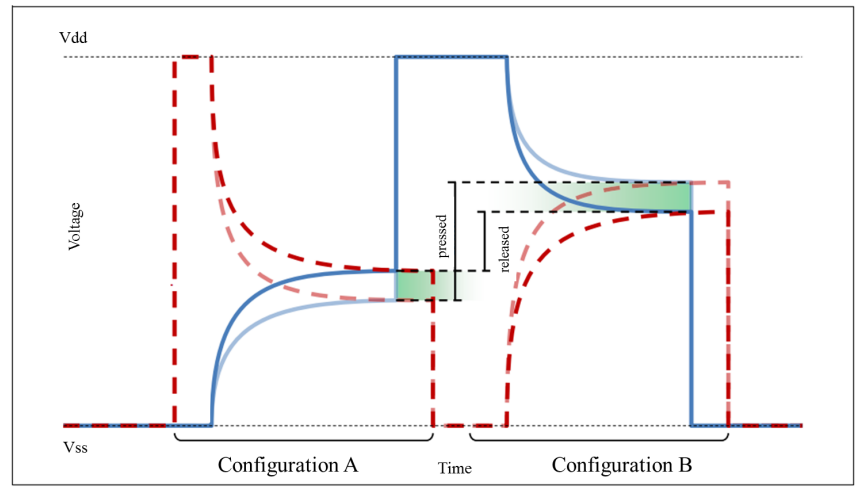

(b) With finger

Figure 4.4: Waveform showing complete operation for Charge Voltage Divider. In (a) without presence of finger, the charges settle in same amount of time which is not the case when there is finger touching the panel (b). 


\subsubsection{Charge Transfer}

In this technique, transfer of charge takes place from one location to another through a series of switches. Using charge transfer technique, capacitance can be measured by accumulating charge from sensor capacitors onto an integrating capacitor. Once accumulated, the potential across the capacitor is measured against reference voltage to calculate the charge across capacitive sensors.

The following graph (Figure 4.5) shows charge integration over time for an example sensing network.

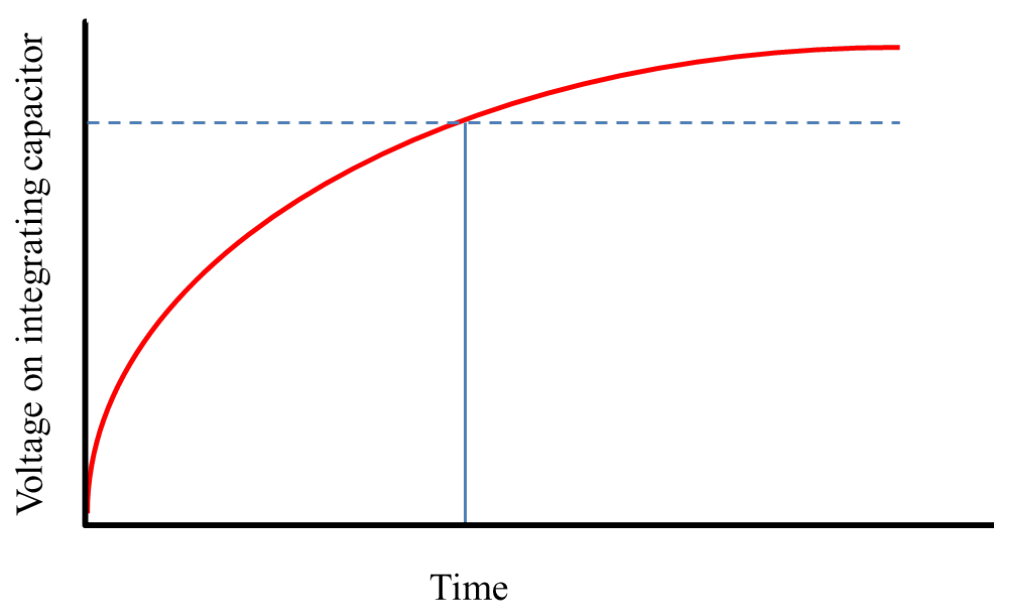

Figure 4.5: Graph showing voltage accumulated over time for charge transfer technique.

When the reference voltage is reached (shown as dotted line in Figure 4.5), the time to achieve this is measured by the controller. This sensing method is robust and has a high signal to noise (SNR) ratio $[9]$.

The focus of this chapter is to express capacitive touch sampling as a mathematical problem. Figure 4.6 shows a very simplified block diagram depicting the working of touch controller. Each intersection of the grid panel has a characteristic mutual capacitance $C_{s}$. The charge transfer sampling process may be restated as follows:

1. A touch panel is selected with $N$ rows and $N$ columns. 


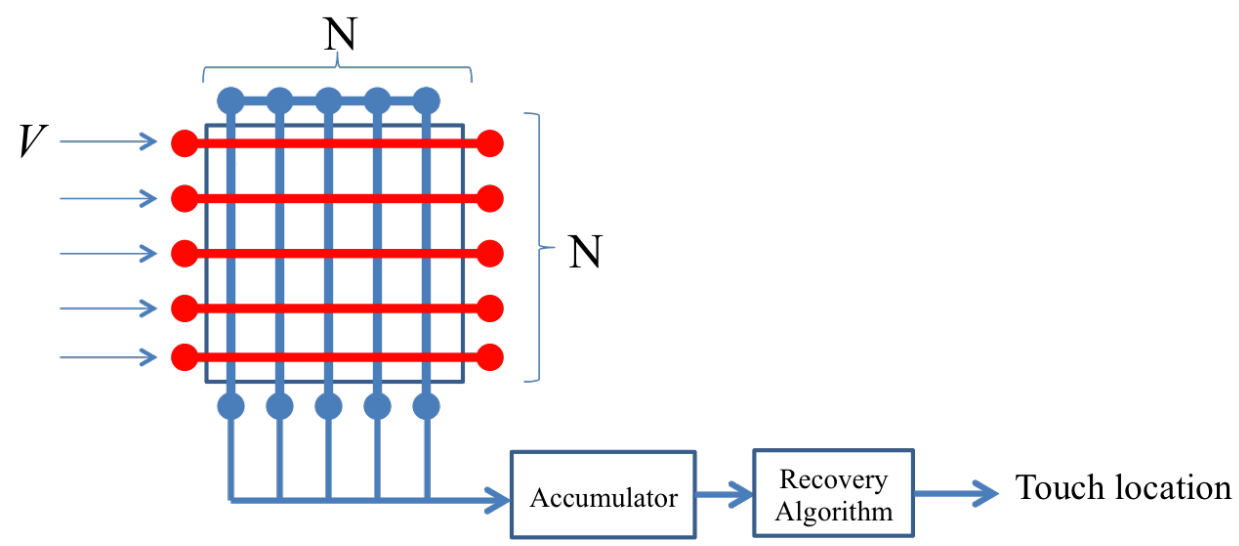

Figure 4.6: Block diagram explaining the measurement process of a capacitive touch controller. A square $N \times N$ panel is shown, although panels can be rectangular as well.

2. The first column of the panel is pre-charged with voltage values taken from an $N \times N$ sampling matrix.

3. The capacitance values on the first column of the panel are accumulated and passed to the recovery algorithm.

4. The algorithm recovers the capacitance values on each node of the touch panel column.

5. The process is repeated for each column of the touch panel.

As opposed to image sensors, which also have a two-dimensional grid, only rows in a capacitive touch panel can be excited and the columns are used for sensing purposes. Sampling of a single column in a touch panel with $N$ intersections traditionally requires $N$ measurements, which induces latency with a larger panel [82, 83].

\subsubsection{Related Work in Efficient Sampling for Capacitive Touch Panels}

The charge transfer sampling process can be represented using a system of linear equations stated below:

$$
\mathbf{v}=\mathbf{A c}+\mathbf{e},
$$


where $\mathbf{c}$ is an unknown $N \times 1$ capacitance vector, $\mathbf{A}$ is an $N \times N$ sampling matrix containing driving voltage values $V$ applied to rows of the panel, $\mathbf{v}$ is an $N \times 1$ measurement vector and $\mathbf{e}$ is the noise vector. This system of equations can be solved easily when $A$ is a square matrix, thus making signal recovery a trivial process. However, doing $N$ number of measurements on a large scale panel is not feasible. As an example scenario, if a touch panel of size $S_{a} \times S_{b}$ inch $^{2}$ is covered by capacitors of individual diameter $d \mathrm{~mm}$, approximately $\frac{25.4 S_{a}}{d} \times \frac{25.4 S_{b}}{d}$ capacitors will be required for the whole panel. The diameter of a single capacitor should be kept smaller than normal finger diameter in order to achieve high accuracy [58], and thus is normally taken between 5-6 mm [84]. Without loss of generality, it is assumed that $S_{a}=S_{b}=50$ inch and $d=5 \mathrm{~mm}$, which results in a requirement of 64516 capacitors to cover the whole panel. Scanning rates in current touch panels range from 20 to 200 $\mathrm{Hz}$ for a smartphone sized touch panel (4-5 inch) with 150-200 capacitors embedded in it [26]. Meanwhile, a large size touch screen ( $\geq 50$ inch) will have more capacitors and therefore it will have much longer sampling time [82]. As a naive extrapolation, if we take a 4 inch panel into consideration and extrapolate its sampling rate for a 50 inch touch panel, the scanning rate per panel will reduce to $8.8 \mathrm{~Hz}$ which is unacceptable for high-end, fast input user applications. Therefore it can be safely stated that a faster sampling method is needed for large scale touch panels.

Traditionally, two approaches are used to counter the increased number of sensors in large panels; one approach is to increase the scanning rate and use parallel sampling for columns on the panel. This approach works with controllers that deploy a lot of multiplexers in order to make the parallel sampling work. Another approach is to increase the number of ADCs used in the sampling circuit. More number of ADCs result in a larger temporal resolution of the panel but both approaches increase the complexity of the analog circuit and also increase power consumption. The goal is to reduce the sampling time without sacrificing accuracy and increasing power consumption. Thus, it is important to look at software based methods which can recover signals using signal processing techniques at the host processor. To be specific, we are interested in reducing sampling time by doing $M$ measurements 
instead of $N$ where $M<<N$. Recently, few works have been reported regarding this problem. A technique proposed in [85] declares that expanding the sensing bandwidth can result in a better sampling strategy for touch panels. Although this technique reduces the measurement time, the data processing cost is increased considerably. Also it demands more low pass filters, modulators and demodulators to be embedded in the analog front end of the system. In [82], concept of partial scanning frames is introduced. After a full scan has been completed, partial scanning regions are identified and only the areas surrounding these regions are subjected to scanning frames. The idea is neat as it decreases the overall scanning time of the panel but the hardware cost and power consumption is quite high because the whole traditional sampling circuit has to be remodeled as highly-parallel hardware components.

In order to reduce sampling time, a non-adaptive group testing (GT) approach has recently been proposed in [86] and [87] to recover touch inputs on a panel using a $M \times N$ Hamming Matrix and $M<N$. The Hamming matrix used is obtained by combining an $N \times N$ Hadamard $\mathbf{H}$ and an identity matrix $\mathbf{I}$ using the matrix operation $(\mathbf{H}+\mathbf{I}) / 2$. This approach only works for single touch and in order to extend the technique to multi-touch, $M$ would be made larger than $N$ rendering the technique inefficient [86]. As stated above, we are interested in an over complete dictionary where $M<<N$ in order to increase the sampling rate. This requires an efficient sampling and recovery technique without compromising accuracy.

\subsubsection{Compressive Sensing for Capacitive Touch Panels}

Compressive sensing is a well-established concept in signal processing where the main idea is that sparse signals and images can be reconstructed using fewer data (or measurements) than what are usually considered necessary by Nyquist-Shannon sampling rate $[88,89,90]$. The major requirement of the compressive sensing problem is the sparsity of the measurement signal. A signal is considered sparse if only a small number $(k)$ of values in the input signal are non-zero and the rest are all zeros. 
In (4.1), $\mathbf{c}$ is the $N \times 1$ sparse signal with $k<<N$ non-zero values. In this scenario, the sparse signal is the capacitive touch signal generated when finger touches the panel. Subtracting the baseline capacitance from c will result in a sparse signal because generally the number of fingers on the panel is much smaller than the total number of sensors embedded on it. The baseline capacitance represents the steadystate no-interaction capacitance seen by the touch controller. It is the sum of all the parasitic capacitance on the touch panel including the electrodes, copper traces, the solder pads and any capacitance associated with the whole circuit (normally falls in the range of few pico-Farads). The vector, which is received at the processor is the $N \times 1$ measured signal $\mathbf{v}$, and consists of accumulated voltage values. The host processor has to derive $\mathbf{c}$ from $\mathbf{v}$ by taking inverse of $N \times N$ matrix $\mathbf{A}$. The matrix $\mathbf{A}$ is called sampling or measurement matrix and its simplest form is the identity matrix, where each row of the touch panel can be driven one by one. When $\mathbf{A}$ is of full rank, it can be titled as a complete dictionary and when its dimensions are $M \times N$, where $M<N$, it is referred to as overcomplete or redundant dictionary. In some compressive sensing applications, the signal has to be converted into a sparse signal using a basis function. Capacitive touch sensing is a unique application where the input signal is already sparse, thus making it a suitable application for compressive sensing. More details on these characteristics of capacitive touch signals is given in later sections.

If the sampling matrix is realized as an over complete dictionary, the signal recovery problem can be stated as:

$$
\min \|\mathbf{c}\|_{0} \quad \text { s.t. } \quad \mathbf{v}=\mathbf{A c}+e,
$$

where $\|.\|_{0}$ denotes the $\ell_{0}$ norm of $\mathbf{c}$. By definition,

$$
\|\mathbf{c}\|_{0}=\sqrt[0]{\sum_{i=1}^{n} c_{i}^{0}}
$$


Going by (4.3), $\ell_{0}$ norm can be classified as a pseudo-norm as it does not satisfy the homogeneity property and is tricky to work with because of the presence of zeroth-power and zeroth-root in it. Each positive signal value will become equal to one, so in the end, it is just a cardinality function which counts the total number of non-zero elements in c. The problem can be stated as: Given the conditions in (4.2), and with A being an over complete dictionary, find the sparsest possible value of $\mathbf{c}$.

Unfortunately, minimizing $\ell_{0}$ norm is not easy. The lack of proper mathematical definition for $\ell_{0}$ norm makes the problem both NP-hard and non-convex which makes it impossible to solve without the use of brute force [91]. But, the beauty of compressive sensing is that with $M<<N$, measurements, the exact signal c can be recovered, if the problem can be rewritten as:

$$
\min \|\mathbf{c}\|_{1} \quad \text { s.t. } \quad \mathbf{v}=\mathbf{A c}+e,
$$

or

$$
\min \|\mathbf{c}\|_{1} \quad \text { s.t. } \quad\|\mathbf{v}-\mathbf{A c}\|_{2} \leq \eta, \quad\|\mathbf{c}\|_{0} \leq k,
$$

where $\|\cdot\|_{1}$ is the $\ell_{1}$ norm of $\mathbf{c}$, which mathematically can be written as $\|\mathbf{c}\|_{1}=$ $\sum_{i=1}^{n}\left|c_{i}\right|$ (sum of signal absolute values), $\|\cdot\|_{2}$ is the $\ell_{2}$ norm (sum of squares of signal values), $\eta$ is the bound for measurement error and $k$ is the sparsity of the touch signal. Hence the updated convex problem is to minimize the $\ell_{1}$ norm of the signal instead.

Much work has been done on compressive sensing, particularly in the field of sampling matrix construction. In [89], it was stated that using a random Gaussian or Bernoulli matrix is an optimal strategy for reconstructing sparse signals from incomplete measurements. In $[92,93,94]$, it was proposed that deterministic matrices such as Toeplitz or circulant matrix can also recover sparse signal with high probability. 
Once the sensing matrix has been selected, the next step is to reconstruct the unknown signal using a recovery algorithm. There are three types of recovery algorithms:

1. Convex optimization and relaxation techniques. These type of methods involve solving a convex program such as the one given in (4.4) using interior point methods [89] and projected gradient methods [95].

2. Greedy algorithms including Matching Pursuit (MP) [96], Orthogonal Matching Pursuit (OMP) [97, 98, 99] and Compressive Sampling Matching Pursuit (CoSaMP) [100]. Greedy algorithms build up to the minimizer of the convex program step by step in an iterative process.

3. Combinatorial algorithms which support rapid reconstruction via group testing methods. These include Fourier sampling [101] and chaining pursuit [102].

From embedded systems point of view, combinatorial algorithms are fast and simple to implement but require highly structured matrices and a much larger set of measurements which may not be feasible in certain applications. Convex relaxation techniques tend to be computationally intensive [103]. Greedy algorithms are intermediate in terms of implementation complexity, but they also require a Moore-Penrose pseudoinverse least squares operation on every iteration and thus, its implementation is not a straight forward task [104].

To the best of our knowledge, compressive sensing remains a theoretical concept in capacitive touch panels because of the difficulty in implementation. Hence there is a need for efficient reconstruction algorithms using simple, deterministic and structured sampling matrices that can bridge the gap between theory and implementation. In this chapter, an efficient framework based on compressive sensing is proposed for reducing the sampling time for capacitive touch sensing. As opposed to well established techniques for compressive sensing where a random matrix and a general purpose algorithm is used, here, recovery algorithms are designed based 
on the structure of the sampling matrices and by using this technique, it is shown that one can come up with efficient solutions for low memory embedded systems. Our framework is aimed at capacitive touch sensing, as discussed below, but is not limited to that application.

The idea that compressive sensing may be used in capacitive touch applications was first introduced by C. Luo et al. [83]. Expanding on their idea, two simple structured binary matrices for sampling a capacitive touch signal are discussed in the following section.

\subsection{Structured Binary Matrices for Sparse Signal Recovery}

Choosing the right sampling matrix is very important for effective signal recovery. It is possible to recover a $k$-sparse signal c provided the matrix $\mathbf{A}$ satisfies the Restricted Isometry Property (RIP) [89, 105, 106].

\subsubsection{Restricted Isometry Property}

A matrix of size $\mathrm{M} \times \mathrm{N}$ is said to satisfy the restricted isometry property with a constant $R$ if, for every $\mathbf{c} \in \chi^{N}(k):=\left\{\mathbf{c} \in \Re^{N}:\|\mathbf{c}\|_{o} \leq k\right\}$,

$$
R:=\min \delta \text { subject to }(1-\delta)\left\|\mathbf{c}_{T}\right\|_{2}^{2} \leq\left\|\mathbf{A}_{M \times T} \mathbf{c}_{T}\right\|_{2}^{2} \leq(1+\delta)\left\|\mathbf{c}_{T}\right\|_{2}^{2},
$$

provided $k \leq T \leq N$ and $\delta^{\prime} \leq \delta \leq \sqrt{2}-1$, where $\delta^{\prime}$ is the RIP constant.

The condition in (4.6) is equivalent to requiring that the Gramian matrix $\mathbf{A}_{M \times T}^{t} \mathbf{A}_{M \times T}$ has all its eigenvalues in $[1-\delta, 1+\delta]$, where $\mathbf{A}_{M \times T}$ is a sub matrix of $\mathbf{A}_{M \times N}$ consisting of $T$ selected columns [107]. Basically RIP forces the condition that all $T$ subsets of columns of A should be nearly orthogonal. They cannot be exactly orthogonal because $M<N$. 
RIP is used to establish theoretical performance guarentees when 1) the measurement vector is corrupted with noise or 2) the vector $\mathbf{c}$ is not $k$-sparse. However, RIP is not the only tool used to analyze the performance of a compressive sensing recovery algorithm. The polytope analysis method proposed in [108] and the geometrical functional analysis techniques [109] are also utilized in academia.

Explicitly computing $R$ would require enumerating all subsets of the columns of $A$, forming each Gramian matrix $\mathbf{A}_{M \times T}^{t} \mathbf{A}_{M \times T}$ and calculting its largest and smallest eigenvalues. This method is not faesible except through brute force and never has been used in academic literature [110]. There is not much information available about RIP for deterministic matrices. On the other hand, much is known about eigenvalues of random Gaussian matrices. It is widely accepted that a random Gaussian matrix can recover the signal with high probability [89, 90, 100]. Though later in this chapter, Gaussian random sampling matrix will be used for one of the proposed algorithms, in the current section, it will be shown that simple deterministic matrices can lead to efficient recovery algorithms. The sampling matrix and recovery algorithm are tightly dependent upon each other. This approach can lead to efficient recovery algorithms without the need of using RIP satisfying matrices.

\subsubsection{Compressive Sensing for Augmented Identity Matri- ces (CSAIM)}

The simplest sparse structured matrix $\mathbf{A}$ is the identity matrix, meaning that each row of the touch panel is excited sequentially. In Figure 4.6, if at one instance, only the top row is driven by some voltage value and the resulting capacitance values in the leftmost column are summed up, the result can be directly mapped to the capacitance value at the first node. The same process can be repeated for each node in the touch panel. However, if only $M<N$ rows from the identity matrix are used, then it is not possible to sense any input occurring at indices $n>M$. This issue is remedied by augmenting the identity matrix as follows. Define the $M \times N$ matrix 
A as:

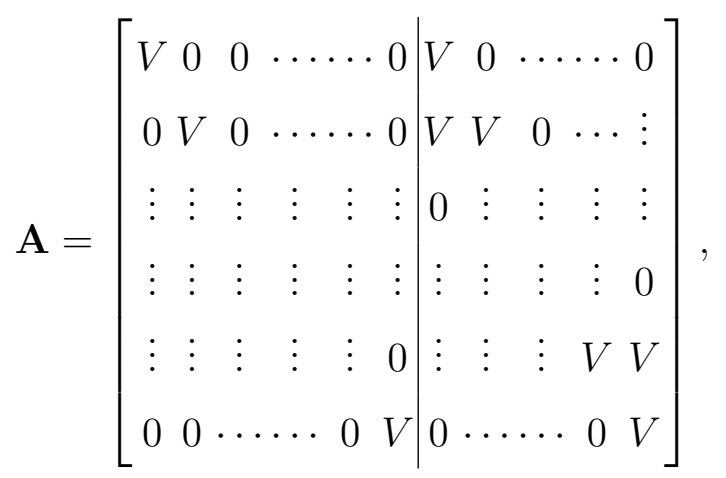

where $N=2 M-1$ and $V$ is the pre-charge voltage. Without loss of generality, $V$ is set equal to 1 in the discussion below. The matrix $\mathbf{A}$ can be defined as a summation of three diagonal matrices of order $M \times N$. It is a sparse and structured matrix that does not need to be stored as it is easily generated.

The augmented identity matrix in (4.7) is a $M \times N$ rectangular matrix. As it will be shown later, it is possible to recover any unknown sparse vector using $M \times N$ measurements, where $\left(\frac{N}{2}+1\right) \leq M<N$. Let us describe a simple algorithm for recovering $\mathbf{c}$, which, unlike traditional greedy algorithms such as OMP, MP or CoSaMP does not require knowledge of the sparsity $k$. The algorithm is titled Compressive Sensing for Augmented Identity Matrices (CSAIM) and makes two assumption for the signal to be recovered $\mathbf{c}$.

1. The non-zero values in $\mathbf{c}$ are non-adjacent to each other by a minimum factor of 2. This means that there are at least two zero values in $\mathbf{c}$ between any two non-zero values. For capacitive touch panels, the algorithm is useful for cases where stylus is used as an input device, as diameter of a stylus is less than or equal to a single sensor on the panel.

2. All non-zero values in the signal $\mathbf{c}$ are positive.

Given both assumptions, and the structure of $\mathbf{A}$ in (4.7), there are only two possible cases for $\mathbf{v}$ values when $k=1$ and four possible cases when $k \geq 2$. These cases are illustrated in Figure 4.7. The cases arising for $k>2$ will be reduced to cases shown 
in Figure 4.7. Justification that there are only limited number of cases is given below, and an algorithm taking advantage of the limited possibilities is described subsequently.

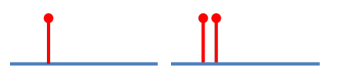

(a) (b)

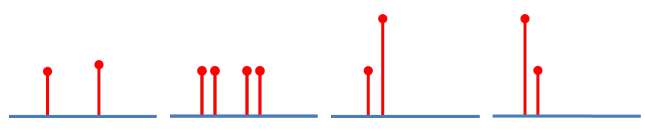

$\begin{array}{llll}(\mathrm{c}) & (\mathrm{d}) \quad(\mathrm{e}) \quad(\mathrm{f})\end{array}$

Figure 4.7: In this figure, (a)-(b) and (c)-(f) represent the two and four possible cases that need to be considered by CSAIM for $k=1$ and $k \geq 2$ respectively.

Consider that the sampling matrix A shown in (4.7) can be divided into two sections column-wise. The first section is an identity matrix and the second is an augmented identity matrix. If we look at the possible areas where the non-zero values in an unknown vector can lie, it can either be in first section or the second section. If a value gets multiplied by the identity matrix, it will be replicated on the correct index in the measurement vector (Figure 4.7(a) and 4.7(c)). On the other hand, if a value gets multiplied by non-zero entries in the second section, they will be shown as duplicates on an incorrect index in the measurement vector (Figure 4.7(b) and $4.7(\mathrm{~d}))$.

Cases (e) or (f) (Figure 4.7(e) and 4.7(f)) can arise if two entries in the unknown signal lie on the same row of the sampling matrix (4.7). As it is assumed that there are no adjacent values in the unknown signal, it can be concluded that no more cases arise for $k>2$ besides the ones explained above.

Algorithm 1 shows the detailed breakdown of all the steps involved in the recovery processes.

Let us look at the rules of recovery for $k \geq 2$ in detail below where $\mathbf{v}$ is the measurement vector and $j$ is the vector index.

CSAIM encounters Case a / Case c: Map the value directly to the output 


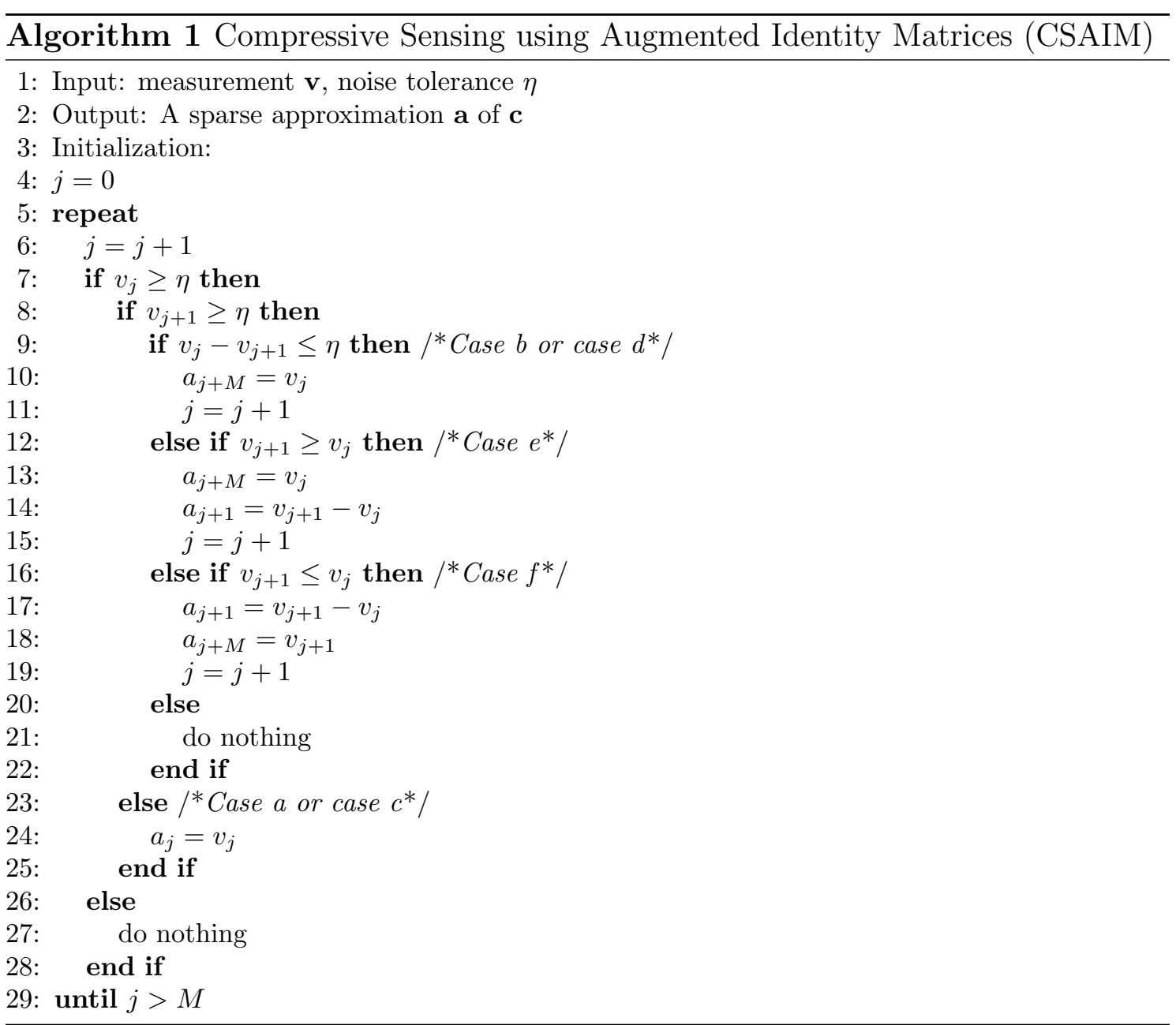

vector a at index $j$.

$$
a_{j}=v_{j}
$$

CSAIM encounters Case b / Case d: Map the value to the output vector a at index $j+M$. Index correction is required as the original system of equations is underdetermined.

$$
a_{j+M}=v_{j} .
$$

CSAIM encounters Case e: Subtract the value $v_{j}$ from its adjacent value $v_{j+1}$ and assign the result to a at index $j+1$. Then map $v_{j}$ to the output vector a at index $j+M$.

$$
a_{j+1}=v_{j+1}-v_{j}
$$




$$
a_{j+M}=v_{j}
$$

CSAIM encounters Case f: Subtract the value $v_{j}$ from its adjacent value $v_{j+1}$ and assign the result to a at index $j+1$. Then map $v_{j+1}$ to the output vector a at index $j+M$.

$$
\begin{aligned}
a_{j+1} & =v_{j+1}-v_{j}, \\
a_{j+M} & =v_{j+1} .
\end{aligned}
$$

The algorithm terminates when all the values in $\mathbf{v}$ have been analyzed. This algorithm neither requires sampling matrix $\mathbf{A}$ to be pre-stored (as it is very easy to generate on the fly) nor requires any knowledge of sparsity $k$.

In order to illustrate workings of CSAIM, we provide the following example:

Example 1: Let $\mathbf{c}$ be the nine-element sparse vector $[0,0,0,1,0,0,0,2,0]$. We compute A using (4.7) with $V=1$ and our measurement vector is given as:

$$
\mathbf{v}=[0,0,2,3,0]
$$

As this is a simple example without presence of noise, the noise tolerance $\eta$ can be set to any small positive constant greater than 0 . for this example, let's take $\eta=0.1$ and the iteration is run for maximum length of $\mathbf{v}$ i.e. 5.

Because the first two values in $\mathbf{v}$ are zero, we will skip them and move to $v_{3}=2$. We will check if $v_{3}$ and $v_{4}$ are both greater than $\eta$ and if their difference is also greater than $\eta$ or not. If so, then we are dealing with either case $e$ or case $f$. Because $v_{4}>v_{3}$, it is case $e$ and as mentioned earlier in algorithm explanation, for case e, we subtract $v_{3}$ from $v_{4}$ and assign its result to an all-zero output vector $\hat{\mathbf{c}}$ at index 4. Then we assign $v_{3}$ to index $3+M$ where $M=5$. Thus, our resulting vector is:

$$
\hat{\mathbf{c}}=[0,0,0,1,0,0,0,2,0],
$$


which is equal to our original $\mathbf{c}$ vector that we were trying to recover.

The limitation of CSAIM is that it imposes on non-zero values in $\mathbf{c}$ to be nonadjacent to each other by a minimum factor of 2 . In the case of capacitive touch sensing, there are two topologies where CSAIM can be used:

1. Capacitive touch panels designed for applications with large sensor spacing topology. Examples may include but are not limited to POS systems and ATM machines. In such devices, the pitch size can be set up equal to the finger size or more, as they do not require a very precise and accurate touch input [83]. For such panels, CSAIM can be an ideal option for efficient sampling.

2. Capacitive touch panel applications that involve use of a stylus instead of finger touch. Normally, the diameter of stylus is smaller than the diameter of sensor regardless of the pitch size. For such applications, it is safe to assume that there will only be one or two sensors triggered at a given time over the whole panel. Drawing, sketching and writing are such applications where use of stylus is necessary.

In case of large-scale touch panels where finger is used and covers multiple sensors, the block sparse nature of the touch signal must be taken into account. This means that the non-zero values will be adjacent to each other. Keeping this in mind, let us introduce a new algorithm for block sparse signals below.

\subsubsection{Block Compressive Sensing using Binary Matrices (BCSBM)}

In capacitive touch sensing, the electrodes are designed such that the finger usually covers adjacent rows and columns on the touch panel. This allows precise subpixel identification of the finger position. It can be stated that in the case of finger touching the panel, all non-zero values will occur in blocks separated by blocks of zeros. Assume, that the finger covers at most $s \times s$ electrodes. Then a block sparse 
sampling matrix may be constructed using the Kronecker product of pre-charge voltage and an identity matrix as shown in (4.14).

$$
\mathbf{A}=\mathbf{I} \otimes \mathbf{V}_{1 \times s}
$$

As an example, for $s=3, \mathbf{I}_{3 \times 3}$ and $\mathbf{V}=[V V V]$, the resulting matrix $\mathbf{A}$ generated using (4.14) is:

$$
\mathbf{A}_{3 \times 9}=\left[\begin{array}{ccccccccc}
V & V & V & 0 & 0 & 0 & 0 & 0 & 0 \\
0 & 0 & 0 & V & V & V & 0 & 0 & 0 \\
0 & 0 & 0 & 0 & 0 & 0 & V & V & V
\end{array}\right] .
$$

The $M \times s M$ matrix is generated easily using a shift register. The block structure can be easily adjusted to use any block size besides three.

The algorithm using $\mathbf{A}$ to recover $\mathbf{c}$ is titled Block Compressive Sensing for Binary Matrices (BCSBM) and its listing is given in Algorithm 2.

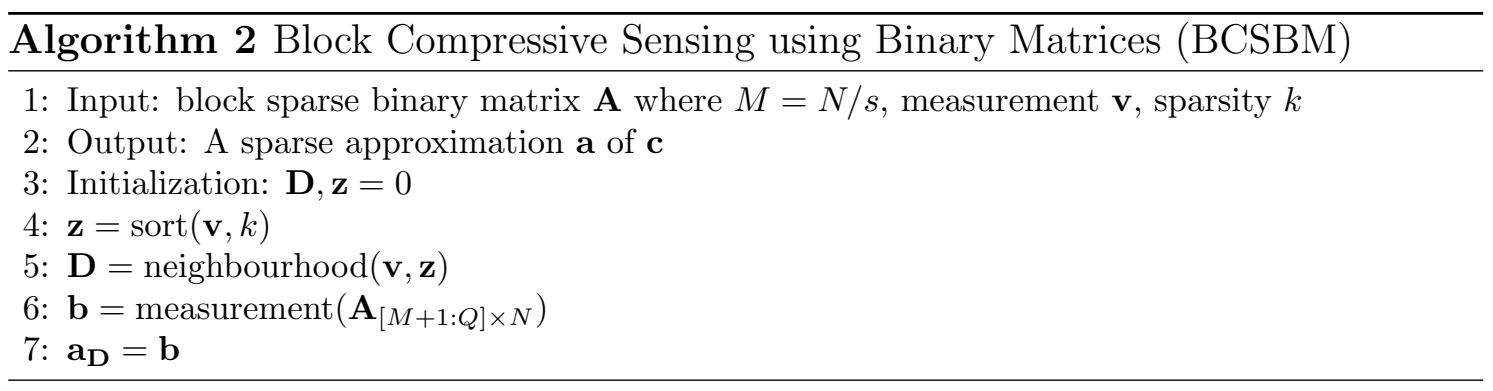

At the start, the measurement vector $\mathbf{v}$ is sorted in descending order using the sort() function in line 4 of Algorithm 2; and the indices of the top $k$ values are stored in separate vector $\mathbf{z}$, where $k$ is the sparsity of the signal.

The neighborhood() function in line 5 is vital for BCSBM. This step is responsible for formation of the support of the estimate. Because of the unique structure of block sampling matrix, it is possible to locate the indices of non-zero values in $\mathbf{c}$ by looking at the values of $\mathbf{v}$. If, for example, $\mathbf{v}$ had its maximum value at index 2 , that means that the second row in $\mathbf{A}$ produced that non-zero value in v. Keeping 
that in mind, we take the indices of the non-zero values in the second row of $\mathbf{A}$ (index 4,5 and 6) and create a row vector spanning these values plus two adjacent indices. In this example, the row vector will be $[3,4,5,6,7]$.

There will be one row vector generated per each non-zero value in $\mathbf{v}$ if that value is greater than the noise threshold. All such row vectors form rows of a support matrix D.

In line 6 , we resample the sparse signal based on the entries in $\mathbf{D}$. It is known that D contains possible indices of non-zero entries of $\mathbf{c}$, thus $Q$ number of extra rows of zeros are added to $\mathbf{A}$ where $Q$ is equal to the total number of unique values in $\mathbf{D}$. Exactly one index per row is set equal to 1 based on the entries in $\mathbf{D}$ and the signal is resampled. The resulting vector $\mathbf{b}$ is then mapped to output vector $\mathbf{a}$ according to the indices stored in $\mathbf{D}$ as shown in line 7 .

In order to illustrate workings of BCSBM, we provide the following example:

Example 2: Let $\mathbf{c}$ be the $N$-element sparse vector with one non-zero block $[0,0,3,4,2,0, \ldots, 0,0,0]$. We compute A using (4.14) with $V=1$ and our measurement vector is given as:

$$
\mathbf{v}=[3,6,0,0,0 \ldots 0]
$$

After sorting and putting $k=1$ first values in $\mathbf{z}$, we have $\mathbf{z}=[2]$. Hence $\mathbf{D}$ can be written as:

$$
\mathbf{D}=[34567]
$$

Based on the values in $\mathbf{D}$, we add $Q=5$ number of rows in the original $\mathbf{A}$ : 


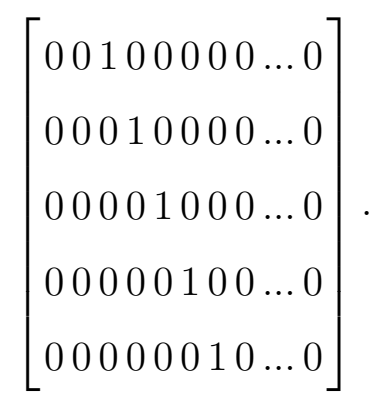

After that, we resample our original unknown signal using the additional rows that we just added. The resulting vector $\mathbf{b}$ is given as:

$$
\mathbf{b}=[3,4,2,0,0]
$$

Vector $\mathbf{b}$ is mapped to the output vector $\hat{\mathbf{c}}$ using the values in $\mathbf{D}$ as represented by line 7 of the algorithm. Hence our resulting vector is:

$$
\hat{\mathbf{c}}=[0,0,3,4,2,0, \ldots, 0,0,0],
$$

which is equal to our original unknown $\mathbf{c}$ vector.

Some noise control comes from the sorting function performed at the beginning of the algorithm. Only $k$ maximum values are kept. As an example, if the measurement vector is $128 \times 1$ in size and $k=2$, using sorting and selection of top $k$ elements will ensure that the random noise added to the rest of the elements in measurement vector are not used in the recovery algorithm. However, the added noise to the first two entries in the sorted vector will not be suppressed. Keeping this in mind, the algorithm can under-perform in the presence of high noise. In capacitive touch sensing, BCSBM can be useful is applications where there is low noise or noise can be removed using simple thresholding methods. Accurate knowledge of $k$ is also required which may not be available all the time, thus posing a limitation on this algorithm. 


\subsection{Greedy Algorithms for Sparse Signal Recov- ery}

Greedy algorithms try to solve compressive sensing problems using linear algebraic tools. They are computationally efficient and perform on similar accuracy levels as the traditional and more complex convex optimization tools. Greedy algorithms take measurement vector as input along with the sampling matrix (sometimes) and try to estimate the support set of the underlying sparse vector. There are two major steps involved in almost all of the Greedy algorithms. A matched filter is used for support set detection and a least squares is used for signal values estimation. Three most popular greedy algorithms are OMP, MP and CoSaMP. After designing simple recovery algorithms which use structured sampling matrices, we wanted to look at greedy algorithms to see if they solve the problem of sampling in capacitive touch panels. We designed a variation of Matching Pursuit called Gramian Matching Pursuit (GMP) and a Block Matching Pursuit with Non-Iterative Least Squares (BMPNILS), which work better than combinatorial algorithms in presence of noise. These algorithms are discussed in detail below.

\subsubsection{Gramian Matching Pursuit (GMP)}

We propose below a variation of MP [96] using rectangular Hadamard matrices as its sampling matrix, but before introducing our proposed algorithm, it is beneficial to define Hadamard matrices in general and how a standard MP algorithm works.

A Hadamard matrix is one whose entries are either +1 or -1 and whose rows are mutually orthogonal. The order of the rows of any Hadamard matrix must be 1, 2 or a multiple of 4 [111]. For simplicity, let us assume $N$ is a power of 2 . Then it is well-known that a Hadamard matrix of order $2^{q} \times 2^{q}$ is obtained recursively using 
Kronecker products, starting with the order 2 matrix

$$
\left[\begin{array}{cc}
1 & 1 \\
1 & -1
\end{array}\right] \text {. }
$$

Let $\mathbf{H}_{2^{q} \times 2^{q}}$ denote the Hadamard matrix constructed in this manner.

Define a restriction matrix containing top $M$ rows of an Identity matrix $\mathbf{I}$ as:

$$
\mathbf{R}_{M \times 2^{q}}=\mathbf{I}_{M \times 2^{q}} .
$$

The standard Hadamard matrix can be restricted in order to obtain rectangular sampling matrix as follows:

$$
\mathbf{A}_{M \times 2^{q}}=\mathbf{R}_{M \times 2^{q}} \mathbf{H}_{2^{q} \times 2^{q}}
$$

The advantage of using Hadamard matrices in GMP will be discussed in detail after explanation of how GMP works. A standard MP algorithm can be stated as follows:

1. Initialize a residual $\mathbf{r}$ equal to measurement vector $\mathbf{v}$

$$
\mathbf{r}^{0}=\mathbf{v}
$$

2. Find out the maximum correlation between atoms of sampling matrix $\mathbf{A}$ and $\mathbf{r}^{j}$, where $j$ is the iteration index.

$$
\begin{aligned}
\mathbf{p} & =\mathbf{A}^{T} \mathbf{r}^{j}, \\
t & =\operatorname{argmax}_{i}\left(\left|\mathbf{p}_{i}\right|\right) .
\end{aligned}
$$

3. Assign the value to the output vector a.

$$
\mathbf{a}_{t}=\mathbf{a}_{t}+\mathbf{p}_{t}
$$


4. Initialize an all zero masking vector $\mathbf{m}$ and assign $\mathbf{p}_{t}$ to it. Update residual using $\mathbf{m}$ and reiterate from step 2 till residual norm $\|\mathbf{r}\|_{2}$ falls below the norm threshold $\eta$.

$$
\begin{aligned}
\mathbf{m}_{t} & =\mathbf{p}_{t}, \\
\mathbf{r}^{j+1} & =\mathbf{r}^{j}-\mathbf{A m} .
\end{aligned}
$$

The GMP algorithm starts off as a normal matching pursuit algorithm apart from one important change; we run normal matching pursuit for the first iteration to get initial inner product between sampling matrix and measurement vector. Once we have the inner product, multiplication step can be skipped to save resources in the following iterations.

This is achieved by exploiting the structure of the Gramian matrix $\left(\mathbf{A}^{T} \mathbf{A}\right)$. The process of matrix vector multiplication in (4.20) is avoided by a simple substitution as shown below.

$$
\begin{aligned}
\mathbf{p}^{j+1} & =\mathbf{A}^{T} \mathbf{r}^{j+1} \\
& =\mathbf{A}^{T}\left(\mathbf{r}^{j}-\mathbf{A} \mathbf{m}\right) \\
& =\mathbf{p}^{j}-\mathbf{A}^{T} \mathbf{A} \mathbf{m} \\
& =\mathbf{p}^{j}-\mathbf{B m} .
\end{aligned}
$$

Equation (4.28) can be rewritten as:

$$
\mathbf{p}^{j+1}=\mathbf{p}^{j}-\mathbf{B}_{[:, t]} u
$$

where $\mathbf{B}_{[:, t]}$ represents the $t$-th column of $\mathbf{B}$ and $u=\mathbf{p}_{t}$.

Once we have our updated $\mathbf{p}$ vector, the next step is to calculate the residual as 
shown below.

$$
\mathbf{r}^{j+1}=\mathbf{r}^{j}-\mathbf{A}_{[:, t]} u
$$

where $\mathbf{A}_{[:, t]}$ represents the $t$ column of matrix $\mathbf{A}$.

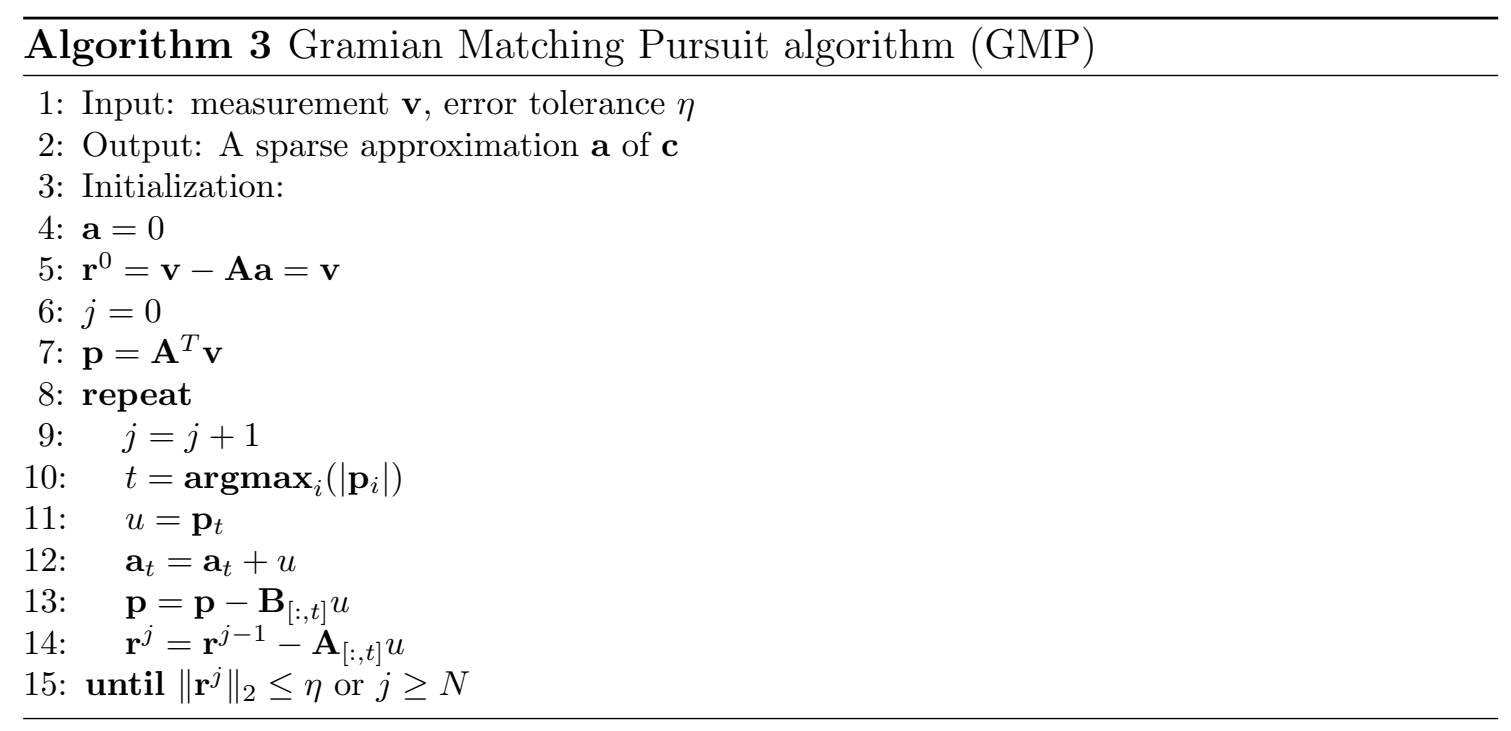

The step by step procedure of GMP is given in Algorithm 3. In the initialization stage, the estimation $\mathbf{a}$ and the residual vector $\mathbf{r}$ is initialized. During the first iteration, vector $\mathbf{p}$ is calculated and its maximum value is assigned to $\mathbf{a}$. In the subsequent iterations, the value of $\mathbf{p}$ is updated. On each iteration a single value is assigned to the output by augmenting the support of the largest entry in p. Line 14 re-evaluates the residual signal so that condition for loop termination can be checked. The loop terminates when the residual energy falls below the error tolerance value. The computation performed during the iterative process is not intensive and thus it can be easily implemented in a low memory embedded system. Unlike the compressive sampling algorithm proposed for capacitive touch in [83], our method does not perform a pseudo-inverse, or carry out a least squares minimization. Furthermore, as the size of vectors remain the same throughout the algorithm runtime, these iterations can be performed in parallel.

Use of a rectangular Hadamard as the sampling matrix A in GMP has two major advantages: 
1. If $\mathbf{A}$ is an $M \times N$ rectangular normalized Hadamard matrix where $M<<N$, then its entries are either $+q$ or $-q$, where $q=\left\|\mathbf{A}_{[:, t]}\right\|_{2}^{-1}$ (or the inverse of the column norm of the matrix) and $t$ can be representative of any column of the rectangular Hadamard. This information reduces the number of multiplications in (4.30) to one.

2. A Hadamard matrix also helps reduce the number of multiplications in (4.29) due to the simple structure of its Gramian matrix. The Gramian matrix $\mathbf{B}=\mathbf{A}^{T} \mathbf{A}$ will always have a block circulant structure with a few distinct values $U$. Further explanation is given below.

By looking at the structure of an $N \times N$ non-normalized Hadamard matrix $\mathbf{H}_{N}$, it can be noticed that barring the leftmost column, it contains exactly $\frac{N}{2}+1$ and -1 values per column. Similar equation holds for the rows barring the top row. Furthermore the columns are orthogonal to each other. This means that the total number of non-zero unique values $(U)$ in $\mathbf{H}_{N}^{T} \mathbf{H}_{N}$ will be equal to 1 as:

$$
\mathbf{H}[:, i]^{T} \mathbf{H}[:, j]=0 \quad \forall \quad i \neq j \quad i, j<N .
$$

When the last row of $\mathbf{H}$ is removed, the number of +1 s and -1 s is no longer equal, thus making the columns non-orthogonal with each other. Equation (4.31) no longer holds true and it can be safely speculated that in this case $U \geq 1$.

If all rows barring the first one are removed, i.e. $M=1$, the Gramian is a $1 \times N$ matrix with all elements equal to 1 , and thus there is only one unique value. As $M$ is increased, for each subsequent row, we are adding equal number of positive and negative values. It can be verified empirically that for a rectangular Hadamard matrix, the upper bound on the number of unique values $U$ for $\mathbf{B}$ is,

$$
U \leq \log _{2}(N)-1 \quad \forall \quad \mathbf{A}_{M \times N},
$$

where $\mathbf{A}$ is a rectangular Hadamard matrix and $M \leq N$. 
GMP can be used with a Toeplitz or circulant [92, 93, 94] or random [89] matrix satisfying RIP. However, for a random Toeplitz or random circulant matrix, upper bound on $U$ is much bigger because apart from the transpose symmetry, all the other values will be unique in the Gramian.

$$
U \leq \frac{N(N+1)}{2} \quad \forall \quad \mathbf{A}_{M \times N}
$$

where $\mathbf{A}$ is a rectangular random Toeplitz or random circulant matrix and $M \leq N$. As an example, for $N=128$, the number of non-zero unique values in the Gramian is equal to 6 and 8256 for rectangular Hadamard and random Toeplitz matrix respectively. If data is represented by 32 bits in the system, this translates to only 24 bytes of storage space required for Gramian of rectangular Hadamard matrix and 33 kilobytes of storage space for Gramian of random Toeplitz or random circulant matrix. The difference in memory requirements is apparent. Thus, in the case of capacitive touch sensing, GMP can be an useful algorithm in cases where there is a large memory constraint on the touch controller side. It is clear from (4.32) and (4.33) that using a partial Hadamard matrix will result in reduction of number of multiplications in (4.29) by a large factor.

In GMP, for a fixed value of $M$, a sparse signal with $k$ non-zero values can be recovered with negligible error if:

$$
k \leq \frac{M}{f \log N}
$$

where $f$ is a small positive constant $[112,113]$.

\subsubsection{Block Matching Pursuit with Non-Iterative Least Squares (BMPNILS)}

In Chapter 3, a 2-D Gaussian function was used to model the finger touching the panel. Capacitance response to a finger touch can also be modeled using a 2-D 
Gaussian:

$$
C(x, y: u, v, \sigma)=C_{b}+C_{p} \exp \left[-\frac{(x-u)^{2}+(y-v)^{2}}{2 \sigma^{2}}\right]
$$

where $C_{b}$ is background or base capacitance, $C_{p}$ is the peak capacitance, varying with finger pressure, and $(u, v)$ is the center of the finger. For convenience, we will assume $C_{p}=1$ in the future.

The capacitive touch signal characteristics are unique and to the best of our knowledge, no one has ever utilized all of these characteristics to propose a greedy algorithm for touch panels. Below we list down some of the more obvious characteristics and see how they can help in designing an accurate and efficient greedy algorithm than the state of the art methods.

\subsubsection{Capacitive Touch Signal Characteristics}

Consider the capacitive touch panel shown in Figure 4.8. It can be noticed that due to finger shape, the capacitor right underneath the finger will report the biggest change in capacitance and the change will decay on the sides. The charge distribution can be represented as a bell shaped curve, and for simplicity reasons, assumed to be a 2-D Gaussian distribution affecting an area covered by $s \times s$ capacitors as already approximated by the model in (4.35).

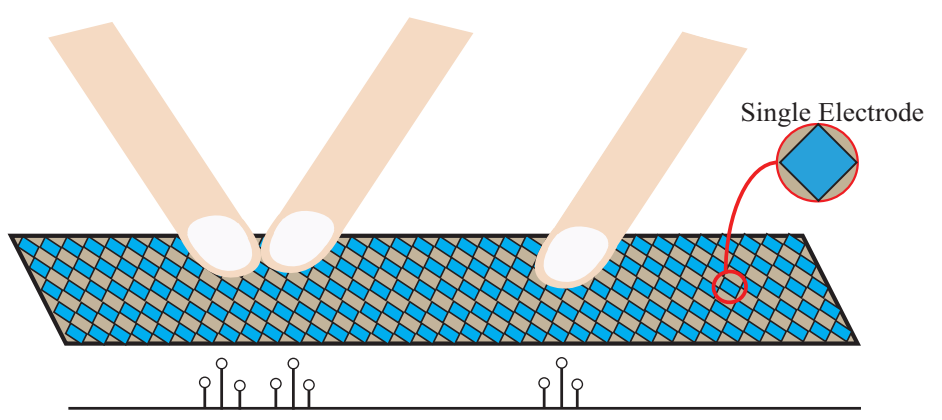

Figure 4.8: Fingers rested on a touch panel and the sparse signal arising from it.

For multiple fingers, there is an air gap between them due to the human finger anatomy as seen in Figure 4.8. The mathematical representation of a capacitive 
touch signal $\mathbf{c}$ for an individual column $n$ of the touch panel with two fingers touching the panel can be represented as:

$$
\mathbf{c}_{n}=[0, \ldots, 0, \underbrace{c_{1}, \ldots c_{s}}_{\text {Finger }}, 0, \ldots, 0, \underbrace{c_{s+1}, \ldots c_{2 s}}_{\text {Finger }}, 0, \ldots, 0]
$$

where $s$ is the largest possible size for an individual block. Equation (4.36) is a representation of a block sparse signal with an assumption that individual blocks are non-adjacent to each other and that each block is roughly Gaussian shaped.

Capacitive touch signals are highly sparse because at any time during operation, the number of fingers touching the panel would be less than the total number of capacitors embedded on the panel by a big margin. As an example, a $50 \times 50$ inch touch panel being used by three people simultaneously with ten fingers each can only have a maximum of 270 capacitors triggered out of 64516 translating to only $0.41 \%$ of the total panel size.

A few greedy algorithms have already been proposed for capacitive touch sampling $[83,114]$. In [83], CoSaMP [100] is adopted for signal recovery which is a general purpose algorithm and does not take into account the prior knowledge about the unique nature and characteristics of capacitive touch signals. In [114] a variation of the matching pursuit (MP) algorithm is presented as a solution to the large scale capacitive touch sampling problem. Similarly, the algorithm does not fully utilize the unique characteristics of the capacitive touch signal. Based on the block sparse nature of touch signal, a number of block-based greedy algorithms, such as BlockOrthogonal Matching Pursuit (BOMP), Block-Matching Pursuit (BMP) [115] and Block-CoSaMP [116] can be adopted for more accurate and efficient recovery. To the best of our knowledge, block based algorithms have never been proposed for touch screen application, despite being more accurate than their non-block based counterparts [115]. We review such algorithms for capacitive touch sampling and propose a modified version of BMP for even higher accuracy and efficiency.

The first step is to select a sampling matrix. Previously, we used deterministic ma- 
trices, but here we want to use a random Gaussian matrix (independent identically distributed (iid)) for BMPNILS. The reason being that unlike other algorithms, now, we are focusing on changes in the BMP algorithm itself in order make it suitable for capacitive touch sensing and are not concerned about designing the sampling matrix. We will touch the topic of sampling matrix design for BMPNILS in Chapter 6.

The matrix can be generated in hardware using a linear feedback shift register (LFSR) [117]. The sampling matrix needs to be an over-complete $M \times N$ dictionary where $M<<N$, and $M$ can be calculated using (4.34). The value of $f$ in (4.34) is normally taken as $\leq 20$ [99]. The log factor ensures that the number of measurements $M$ does not increase considerably with the increase in touch panel size.

\subsubsection{Architectures of Existing Block Sparse Greedy Algorithms}

Once a sampling matrix $\mathbf{A}$ has been generated, $\mathbf{c}$ can be recovered using block sparse compressive sensing techniques. A step by step breakdown of BMP [115] is analyzed in detail as a preparation for introducing other block sparse algorithms and our proposed algorithm.

1. Initialize an iteration index $j=0$ and a support vector $\mathbf{d}$ and set the residual $\mathbf{r}$ equal to the measurement vector $\mathbf{v}$ in $(4.1)$,

$$
\begin{aligned}
\mathbf{r}^{j} & =\mathbf{v}, \\
\mathbf{d}^{j} & =\emptyset .
\end{aligned}
$$

2. Calculate the inner product of the residual $\mathbf{r}^{j}$ with the sampling matrix $\mathbf{A}$,

$$
\mathbf{g}^{j}=\mathbf{A}^{\mathbf{T}} \mathbf{r}^{j}
$$


3. Select index $u$ of the maximum absolute value $\sigma$ in the resulting vector $\mathbf{g}$ and construct a new vector $\mathbf{d}_{\text {new }}$ using the non-zero block containing $u$.

$$
\begin{aligned}
& (u, \sigma)=\max \left(\left|\mathbf{g}^{j}\right|\right), \\
& \mathbf{d}_{\text {new }}=\left\{u-\frac{s-1}{2}, \ldots, u, \ldots, u+\frac{s-1}{2}\right\} .
\end{aligned}
$$

where $s$ is the size of individual non-zero block.

4. Merge $\mathbf{d}_{\text {new }}$ with the support vector $\mathbf{d}$ and update the output vector $\tilde{\mathbf{c}}$ using $d_{\text {new }}$.

$$
\begin{aligned}
\mathbf{d}^{j+1} & =\mathbf{d}^{j} \cup \mathbf{d}_{\text {new }}, \\
\tilde{\mathbf{c}}(t) & \leftarrow \tilde{\mathbf{c}}(t)+\mathbf{g}(t), \quad \forall t \in \mathbf{d}_{\text {new }} .
\end{aligned}
$$

5. Generate a zero valued temporary vector $\mathbf{m}$, and use it for updating the residual.

$$
\begin{aligned}
\mathbf{m}(t) & =\mathbf{g}(t), \quad \forall t \in \mathbf{d}_{\text {new }} \\
\mathbf{r}^{j+1} & =\mathbf{r}^{j}-\mathbf{A} \mathbf{m}
\end{aligned}
$$

6. Increment $j$ by 1 and repeat steps $2-5$ till $j$ equals the maximum number of iterations $J$ or the residual norm value falls below a certain threshold $\eta$.

$$
\left\|\mathbf{r}^{j}\right\|_{2}^{2} \leq \eta
$$

where $J$ can be the maximum number of fingers the touch panel can support. Exploiting block nature of the signal results in higher reconstruction accuracy due to block-wise support selection [115] which also leads to an $s$ times increase in convergence speed.

BOMP and Block-CoSaMP $[115,116]$ tend to recover a signal better than BMP in terms of accuracy. In BOMP, the residual is always orthogonal to the span of 
blocks already selected. This step ensures that the already selected blocks will not be reselected during the remaining run of the algorithm. This is achieved using a least squares operation in every iteration as follows:

$$
\tilde{\mathbf{c}}=\operatorname{argmin}\left\|\mathbf{v}-\mathbf{A}_{\mathbf{d}} \mathbf{c}_{\mathbf{d}}\right\|_{2}^{2}
$$

The output vector $\tilde{\mathbf{c}}$ is updated in every iteration. Block-CoSaMP also uses least squares to update the signal estimates, but it selects multiple blocks instead of one, and filters the already done selection in each iteration [116].

The architectural diagrams of BMP, BOMP and Block-CoSaMP are shown in Figure 4.9. All three algorithms assign output inside the loop and while BMP assigns the output directly; BOMP/Block-CoSaMP use least squares.

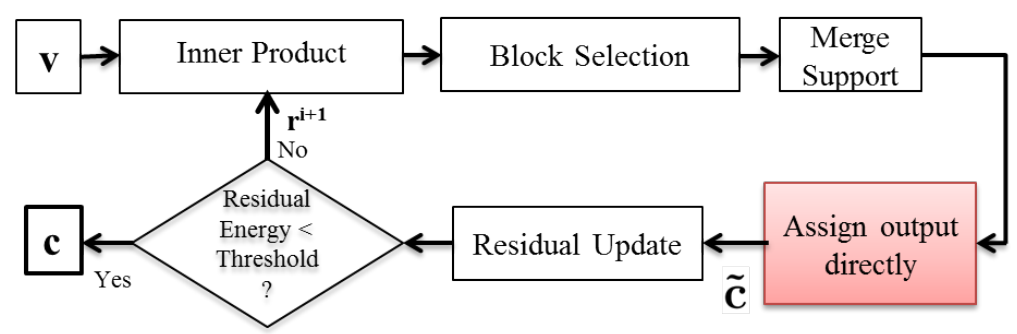

(a)

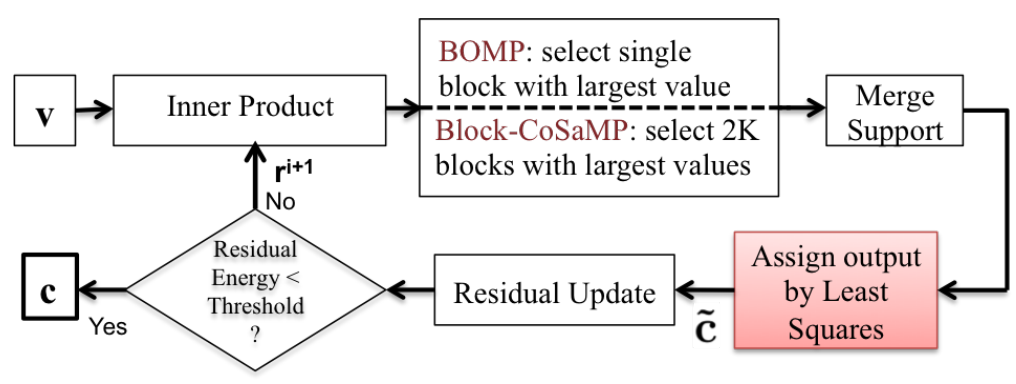

(b)

Figure 4.9: Architectural diagrams for (a) BMP (b) BOMP/Block-CoSaMP showing all the steps involved for signal recovery.

\subsubsection{BMPNILS Algorithm Listing and Discussion}

After reviewing the state of the art algorithms on block sparse signal recovery, we propose a modified version of BMP to recover the touch signal, from which the finger 
peak location can be detected.

Our algorithm is based on BMP in the sense that it does block selection, but instead of assigning output inside the loop, it does a single non-iterative least squares procedure at the end. Due to this modification, the algorithm is titled Block Matching Pursuit with Non-Iterative Least Squares (BMPNILS). The architectural diagram of BMPNILS is given in Figure 4.10 and the pseudo-code is listed in Algorithm 4. The novelties and advantages of BMPNILS for capacitive touch signals are summarized below and are highlighted in Figure 4.10.

1. Pre-recovery global and column-wise sweeps reduce wasteful sampling. Selected support for one column is transmitted to the next in order to speed up the recovery process.

2. Least squares operation is performed only once at the end of the algorithm, thus saves computation cost.

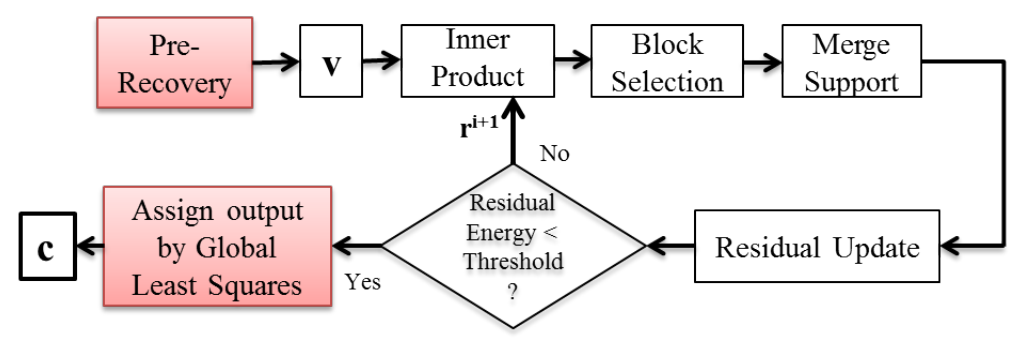

Figure 4.10: Architectural diagram of BMPNILS showing all steps involved in signal recovery.

\subsubsection{Pre-Recovery}

Capacitive touch panels exhibit the following characteristics under normal operation.

1. A high idle to active time ratio because user is not in physical contact with their devices all the time. For this reason modern touch screen controllers implement idle/wake up time periods in order to save power [84]. 
2. A highly sparse signal because only a small portion of capacitors are touched by fingers at a given time.

3. The size of finger is larger than a single electrode, thus similar support can be expected from adjacent columns in the panel.

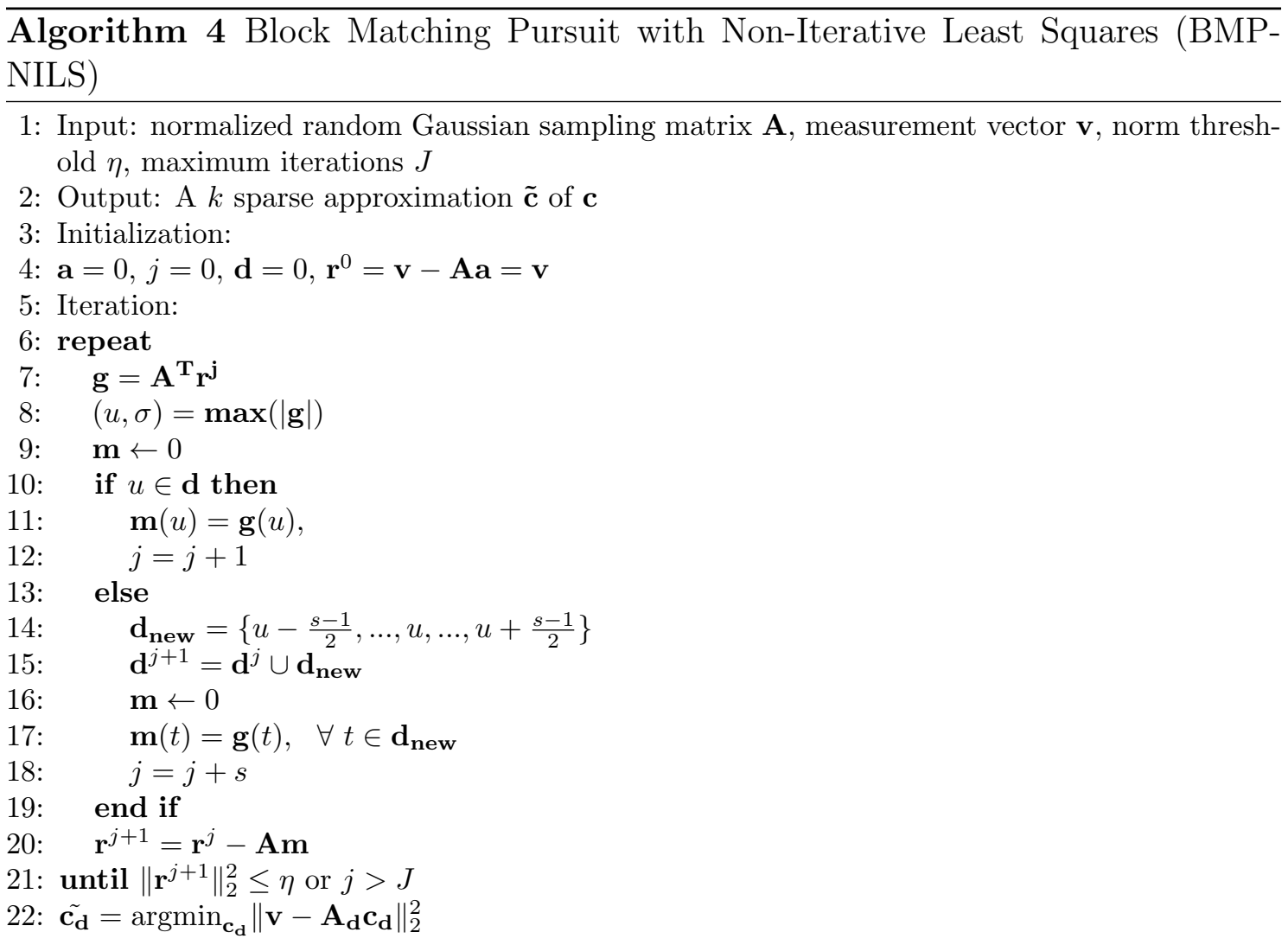

In order to utilize characteristic 1, BMPNILS does a global sweep on the panel by summation of capacitance of the whole panel as follows:

$$
W=\sum_{p=1}^{N} \sum_{q=1}^{N} c_{p q}
$$

where $N$ represents the dimensions of the panel and $c_{p q}$ is the capacitance of an individual node at row $p$ and column $q$. The resulting value $W$ is compared with a constant baseline capacitance to establish the presence of a finger. If there is no finger present, we do not start the recovery process. 
Once a finger is detected on the touch panel, column-wise sweep is performed as:

$$
w_{q}=\sum_{p=1}^{N} c_{p q} .
$$

As a response to characteristic 2 , if $w_{q}$ exceeds a noise threshold, then the recovery process is triggered, otherwise we move on to the next column. This column wise sweep saves time when dealing with highly sparse touch signals.

Due to the block sparsity of capacitive touch signal (characteristic 3), the support vector $\mathbf{d}$ can be pre-populated based on the results of the previous recovery. As an example, if $p$ th column of the touch panel has non-zero values on same indices, this information can be used to pre-populate $\mathbf{d}$ for $(p+1)$ th column. Although pre-recovery seems natural and straightforward, to the best of our knowledge, we have not seen it being proposed in capacitive touch screen literature and therefore it is important to propose pre-recovery in this chapter for future research.

\subsubsection{Global Least Squares}

The second modification to BMP is that, in each iteration, BMPNILS updates the support but does not assign any values to the output vector. Once an accurate support is obtained, the signal is recovered using a single global least squares (line 22 in Algorithm 4). By reducing the number of least squares which is relatively heavy in computation, the computing time can be reduced. The key is then whether such an execution also reduces the recovery accuracy.

The answer hides in the fact that capacitive touch signals are highly sparse. As we see later in simulations, this high sparsity allows a single least squares to perform with similar accuracy as multiple least squares.

In addition to pre-recovery and global least squares, there are a few additional minor modifications in BMPNILS when compared to BMP and are listed below. 
1. As BMPNILS is based on the principle of BMP, there is a lot of re-selection of atoms (columns of $\mathbf{A}$ ) during the iterative process. The algorithm does not add already selected atoms to the support and only updates the residual in the case of re-selection. This minor change ensures that the increment in iteration index $j$ is proportional to the atom selection.

2. Setting an optimal iteration termination criteria is important when implementing sparse recovery algorithms, especially when the exact sparsity value $K$ cannot be predetermined, which is exactly the case for capacitive touch panels. Two different stopping criteria are used in BMPNILS, (1) the maximum iteration index $J$ which is set to be larger than the maximum number of fingers the touch panel can support in order to account for atom re-selection and (2) the residual threshold $\eta$ which is set equal to the $\ell_{2}$ norm of the noise vector added in measurements. The noise level can be tested for each touch panel under various test conditions in order to determine an optimal threshold value.

3. The recovered signal from BMPNILS is subjected to hard thresholding as follows:

$$
\alpha=\operatorname{HT}(\tilde{c}, \zeta)= \begin{cases}\tilde{c}, & \text { if } \tilde{c} \geq \zeta \\ 0, & \text { otherwise. }\end{cases}
$$

which means that a capacitor is considered touched only when the recovered signal $\tilde{c}$ is larger than a threshold $\zeta$.

\subsection{Simulations and Results}

So far, we have proposed four algorithms for efficient sampling in capacitive touch panels. In this section, we will test them with each other and with other block sparse greedy algorithms to see their merits and demerits and comment on when to use them in practical applications. 


\subsubsection{Sampling Matrix Selection}

All algorithms use different sampling matrices. CSAIM and BCSBM use structured binary matrices given in (4.7) and (4.14) respectively. GMP uses a Hadamard matrix and BMPNILS uses a Gaussian random matrix (one whose entries are independent identically distributed(iid)). In order to generate an $M \times N$ Gaussian random, we need to calculate the value of $M$ for a predefined $N$. In our case, $N$ is equal to the number of sensing lines in the panel and $M$ is calculated using (4.34), where $f$ is a small positive constant. The value of $f$ is typically taken as $\leq 20$ [99]. Due to $\log N$, the number of measurements does not increase considerably with the increasing size of touch panel. Figure 4.11 shows the effect of $f$ on Root Mean Square Error (RMSE) and based on the trend, we set $f=3.5$ for $k=10$.

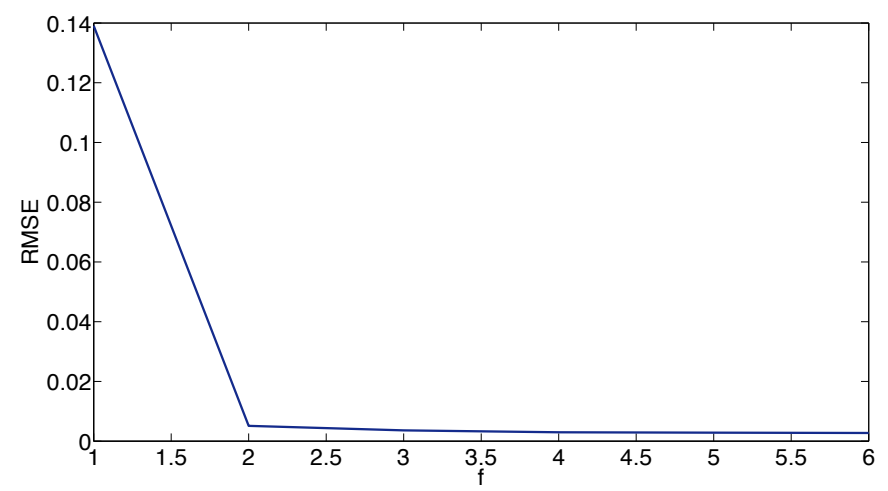

Figure 4.11: RMSE versus $f$, with sparsity $k$ and $N$ kept constant at 10 and 600 respectively.

Figure 4.12 shows recovery of sparse signal using a 30dB SNR measurement vector containing Gaussian noise. The noise vector is generated as a random sequence of normally distributed numbers, which are then multiplied by a noise scale factor. The scale factor is in turn generated proportionally to the variance of the measurement vector. Thus, the variance of the resulting noise vector depends upon the variance of the measurement vector. Accordingly, we use SNR in decibels to represent the amount of noise. 
As seen in Figure 4.12, the algorithms are able to recover the sparse signals accurately. Let us go one step further and compare our algorithms with each other and with state of the art compressive sensing greedy algorithms.
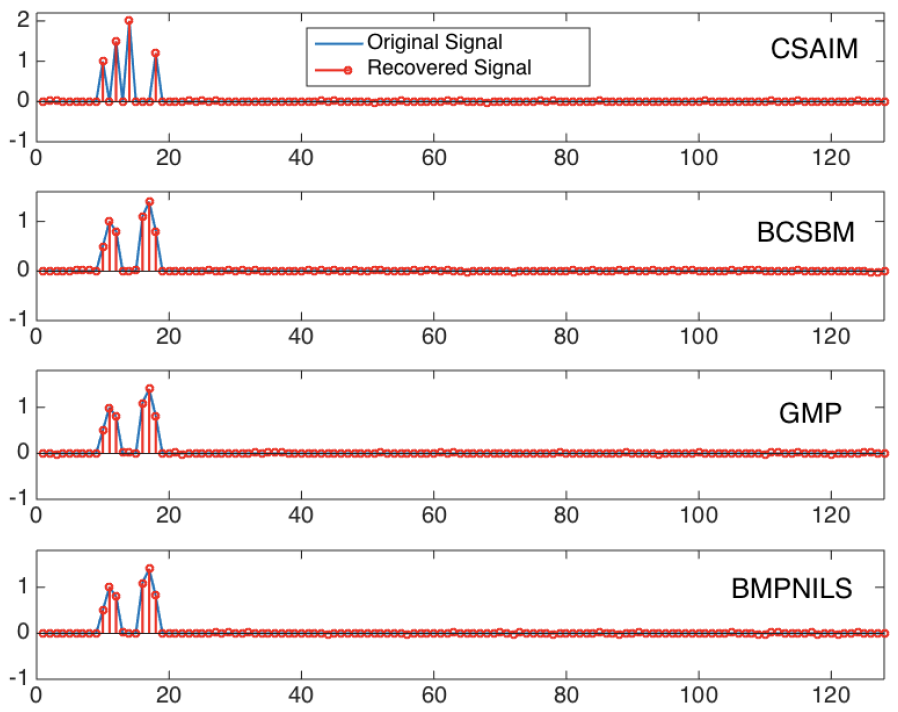

Figure 4.12: Recovery of unknown vector using CSAIM, BCSBM, GMP and BMPNILS with Gaussian noise (figure best viewed in color).

\subsubsection{Recovery Analysis}

In this section, we evaluate the performance of the proposed algorithms for increasing block sparsity levels using a noisy input signal. For each sparsity level, we measure the root mean square error (RMSE) which shows the difference between values estimated by the signal recovery algorithm versus the actual values.

First we compare the proposed algorithms amongst each other. Figure 4.13 shows the RMSE vs sparsity comparison between CSAIM, GMP, BCSBM and BMPNILS algorithms when sampling an $N \times N$ touch panel with 30dB SNR additive Gaussian noise added to the measurement process. We wish to simulate a large-scale $80 \times 80$ inch touch panel and if the pitch of single sensor is assumed to be $5 \mathrm{~mm}, N$ comes out to be 400. The number of fingers $F$ is increased from 1 to 10 (a single person using the panel with both hands). 


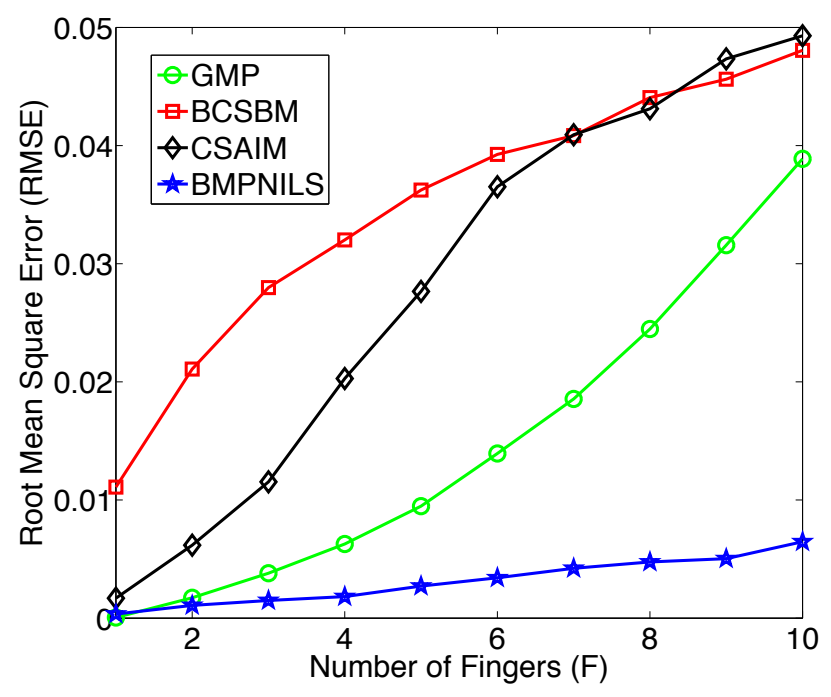

Figure 4.13: RMSE versus number of fingers $F$ for CSAIM, GMP, BCSBM and BMPNILS. $F$ is increased from 1 to 10 while keeping $N$ constant at 400. (figure best viewed in color)

As seen in Figure 4.13, BMPNILS outperforms other algorithms by a big margin. It gives accurate results even in the presence of noise. The reasoning is simple, BMPNILS is based on a variation of BMP whereas GMP is a simple variation of the MP algorithm. It was shown in [115], that BMP is more accurate than MP algorithm for block sparse data and similarly we see that the same results hold for variations of MP and BMP. As CSAIM and BCSBM are non iterative recovery algorithms, their noise filtering process is lacking and hence they under perform in the presence of noise. These algorithms are suitable for low noise conditions and where noise can be removed by global thresholding. In capacitive touch systems where analog noise filtering is proposed $[81,68]$, these two algorithms can be used to sample the filtered signal. We conclude that BMPNILS is the most accurate algorithm out of the four algorithms proposed.

Second, we compare RMSE of GMP and BMPNILS with that of already proposed greedy algorithms for capacitive touch panels. So far, only CoSaMP [83], variation of iterative hard thresholding [118] titled Support Constrained Positive Hard Thresholding (SCPHT) and a variation of MP titled Support Constraint Positive Matching Pursuit (SCPMP) [114] have been proposed for capacitive touch sampling. 
We left out BCSBM and CSAIM from the simulation as they are non-iterative nongreedy algorithms. Figure 4.14 shows the RMSE comparison among BMPNILS, GMP, SCPMP, SCPHT and CoSaMP while sampling an $N \times N$ touch panel with 30dB SNR additive Gaussian noise added to the measurement process. Same as before, the number of fingers $F$ is increased from 1 to 10 (a single person using the panel with both hands) and $N$ is kept constant at 400 .

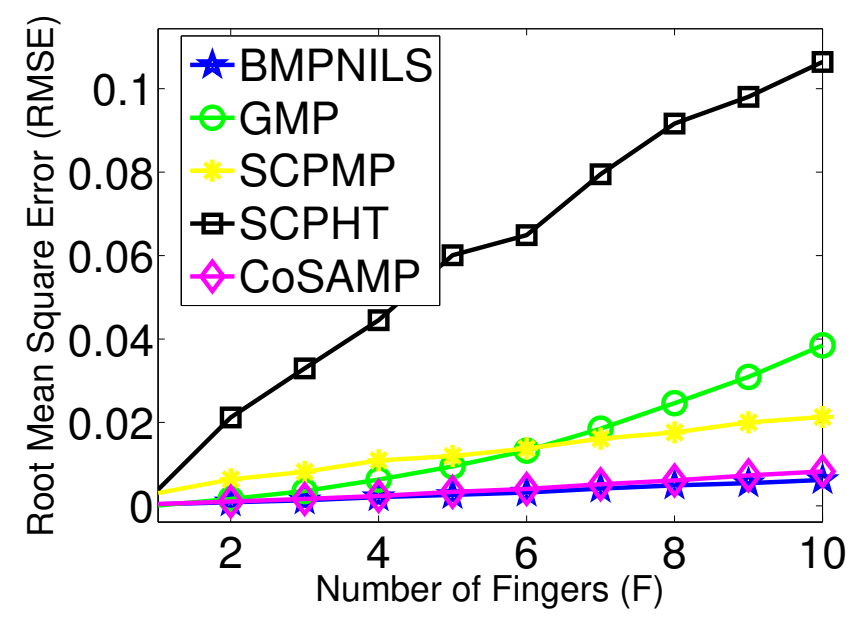

Figure 4.14: RMSE versus number of fingers $F$ for BMPNILS, GMP, SCPMP, SCPHT and CoSaMP. $F$ is increased from 1 to 10 while keeping $N$ constant at 400 (figure best viewed in color).

Figure 4.14 shows that GMP accuracy is comparable to SCPMP at low sparsity levels because both algorithms are similar in the sense that they are both variations of MP algorithm. The advantage of GMP is that it is faster as we will see in the efficiency analysis later. On the other hand, BMPNILS accuracy is comparable to CoSaMP which is an interesting result. BMPNILS is a variation of BMP which itself is based on MP. It has been already proven that CoSaMP is more accurate than the original MP [100]. However, it can be inferred from Figure 4.14, that utilizing the block sparsity nature of capacitive touch signals can lead to an increase in accuracy for simple MP based algorithms.

Third, we compare block sparse based BMPNILS with other state of the art block sparse greedy algorithms. Because our signal has block sparse characteristics, we wish to provide recovery analysis of multiple block sparse greedy algorithms. Figure 
4.15 shows the RMSE comparison among BMPNILS, BOMP, BMP and BlockCoSaMP while sampling an $N \times N$ touch panel with 30dB SNR additive Gaussian noise added to the measurement process. The testing process is same as before but this time a wall display is simulated. Taking the height and width of the panel equal to 3 meters which is approximately equal to the average height of a storey, the size of the wall display will be equal to an $118 \times 118$ inch panel. This panel is simulated with the scenario of three people using the display at the same time. Consequently, the number of fingers $F$ on the panel is increased from 1 to 30 . The sampling matrix is equal to $M \times N$ where $\mathrm{N}$ is equal to 600 assuming pitch size of the panel to be $5 \mathrm{~mm}$. The touch signal to be recovered is block sparse and is generated based on (4.36). Each individual block is referred to as a finger $F$.

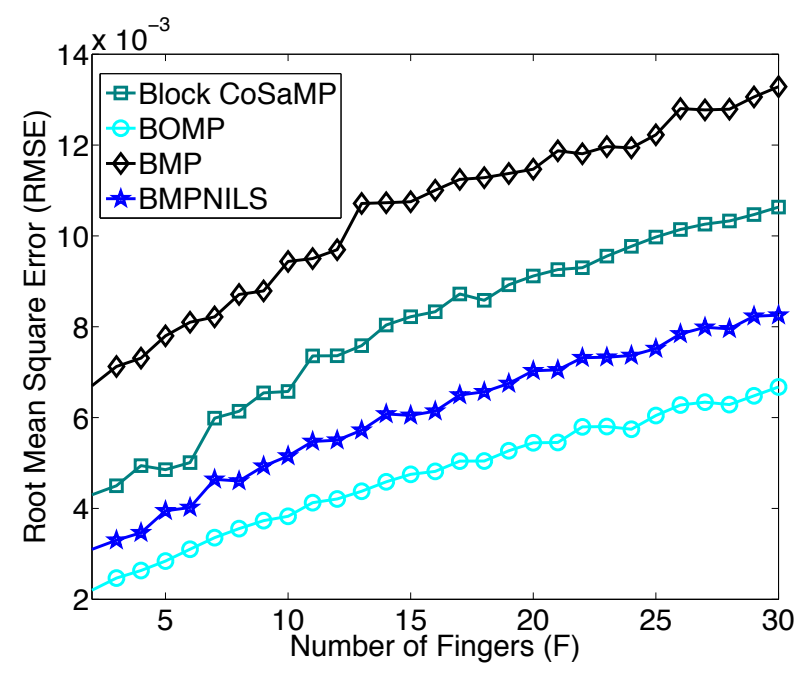

Figure 4.15: RMSE versus number of fingers $F$ for BMPNILS, BOMP, BMP, BlockCoSaMP. $F$ is increased from 1 to 30 (three people using the panel at the same time) while keeping $N$ constant at 600 (figure best viewed in color).

It can be seen in Figure 4.15 that BMPNILS is more accurate than BMP, and is comparable to Block-CoSaMP and BOMP. The popular conventional MP and OMP are also tested and found to be less accurate than BMPNILS, but not shown in the figure to make it clearer for viewing. 


\subsubsection{FLOPS Analysis}

FLOPS (FLoating-point Operations Per Second), is a measure of computer performance. We are using FLOPS as a measure here to show the performance improvement of proposed algorithms while comparing them with other greedy algorithms.

Figure 4.16 shows the number of FLOPS required for all the major operations performed in each proposed algorithm to recover a sparse vector, whose sparsity value is increased from 1 to 10 . It can be observed easily that as the number of fingers increase, BMPNILS requires a larger number of FLOPS than the other three algorithms. The reason is the least squares operation at the end of the algorithm. Another interesting thing to note here is that the number of FLOPS required for the other algorithms do not increase much as the number of fingers is increased. Similar to previous simulations, this simulation is run with additive random measurement noise.

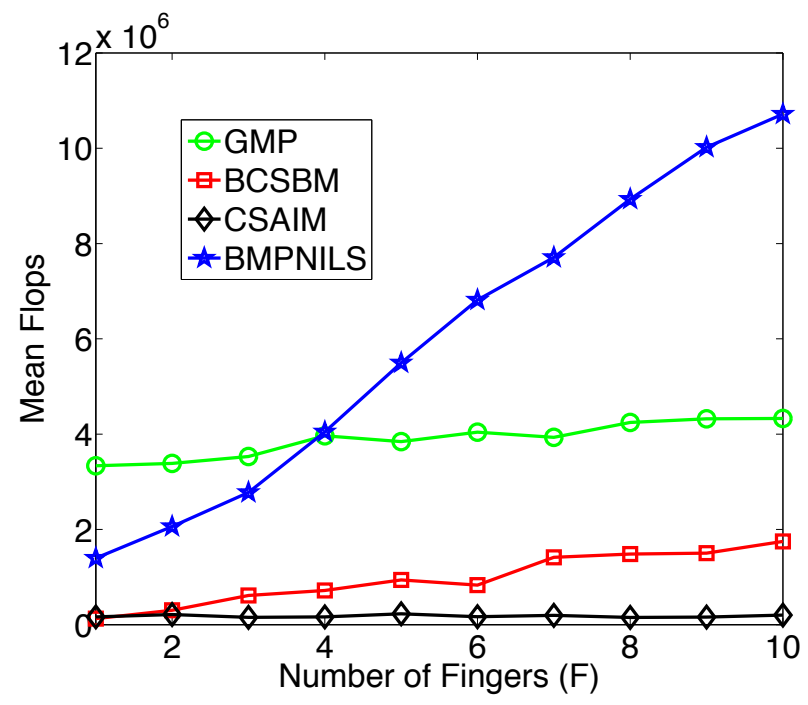

Figure 4.16: Comparison of number of FLOPS required between CSAIM, GMP, BCSBM and BMPNILS. In this figure, the number of fingers touching the panel is changed from 1 to 10 while keeping $N$ constant at 400 (using a $80 \times 80$ touch panel). Figure is best viewed in color.

Next, we compare GMP and BMPNILS with already proposed greedy algorithms for capacitive touch panels for efficiency. Figure 4.17 shows that GMP is faster than 
SCPMP because of its use of Hadamard as the sampling matrix. On the other hand, BMPNILS is much faster than its counterpart CoSaMP. Based on Figure 4.17 and 4.14 , it can be stated that,

1. BMPNILS provides a comparable accuracy to CoSaMP but is more efficient.

2. GMP provides comparable accuracy to SCPMP but is more efficient. The difference in performance between SCPMP and GMP will be more evident when the algorithm is implemented in a low memory touch controller for a practical application.

3. SCPHT is the fastest greedy algorithm ever proposed for capacitive touch panels, but suffers from high estimation inaccuracies.

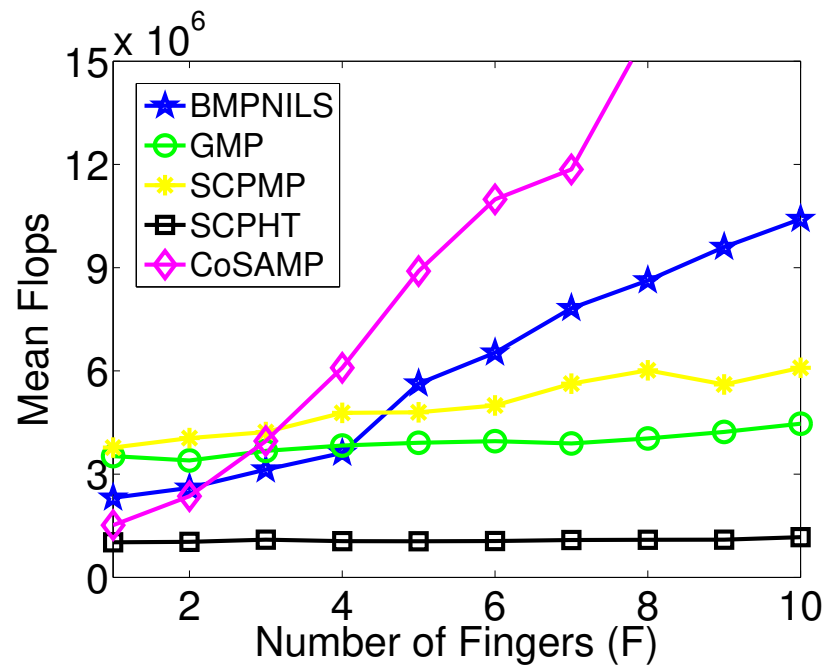

Figure 4.17: Comparison of number of FLOPS required between BMPNILS, GMP, SCPMP, SCPHT and CoSaMP. In this figure, the number of fingers touching the panel is changed from 1 to 10 while keeping $N$ constant at 400 (using a $80 \times 80$ touch panel). Figure is best viewed in color.

Figure 4.18 shows the FLOPS comparison among BMPNILS, BOMP, BMP and Block-CoSaMP when sampling an $N \times N$ touch panel with 30dB SNR additive Gaussian noise added to the measurement process. Because BMPNILS is a block sparse based greedy algorithm, we wish to see how it stacks against other block sparse greedy algorithms. In the figure, initially all algorithms are performing at the 
same speed but BOMP and Block-CoSaMP become slower as $F$ increases. This is because of the increasing number of sampling matrix atoms involved in least squares operation. On the other hand, BMPNILS and BMP maintain their high efficiency even when dealing with high sparsity values. The minor difference between speeds of BMP and BMPNILS is due to the least squares performed at the end of the algorithm. BMPNILS's pre-recovery step is not included in the simulations thus keeping the comparison fair. The simulation is run on an Intel Core i7 $2.8 \mathrm{GHz}$ CPU with $8 \mathrm{~GB}$ of RAM. As a comparison, for 25 fingers, BMPNILS is 0.0170 seconds faster than BOMP and has a difference of approximately 0.001 in RMSE. The computation time difference will be more significant once these algorithms are adopted by a touch controller with a lower processing power. In order to generate Figure 4.16- 4.18, we first calculated the FLOPS constant for our machine and the mean time required per recovery per algorithm, which was then divided by FLOPS constant to find out total number of FLOPS required per algorithm.

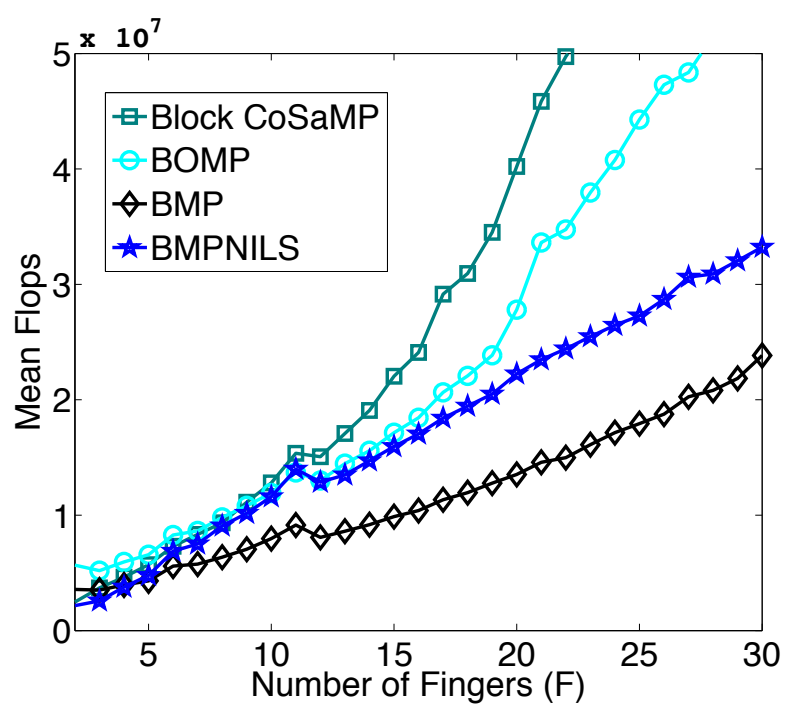

Figure 4.18: Comparison of number of FLOPS required between BMPNILS, BOMP, BMP and Block-CoSaMP. In this figure, the number of fingers touching the panel is changed from 1 to 30 (three people using the panel at the same time) while keeping $N$ constant at 600 (We are using a $118 \times 118$ inch touch panel). Figure is best viewed in color.

Based on Figure 4.13-4.18, the following conclusions can be drawn: 
1. All proposed sampling algorithms in this chapter provide faster sampling and recovery options than the state of the art block sparse greedy algorithms.

2. BMPNILS is the most accurate algorithm out of the ones proposed whereas CSAIM is the fastest in terms of mean FLOPS required.

3. For applications with low noise and when using a stylus, CSAIM is a good choice for a large-scale touch panel.

4. For applications involving low noise and when using a finger, both GMP and BCSBM can be used to save sampling time in large touch panels.

5. For applications with high presence of noise, BMPNILS can be used for speedy and accurate recovery.

We can calculate the power consumption by the touch panel for each recovery algorithms when implemented in an embedded hardware using the expression below.

$$
P=(M N E) F
$$

In (4.51), $P, F$ and $E$ are the power consumption, scanning rate and energy spent on measuring the capacitance of an electrode for a touch panel respectively. For an $118 \times 118$ inch touch wall display, if we take total energy spent on measuring each sensor as $1 / 30 \mu \mathrm{J}[83]$ and a standard scanning rate of $75 \mathrm{~Hz}$, then the power consumption for the proposed algorithms for sampling 10 fingers simultaneously is given in Figure 4.19.

As seen in Figure 4.19, the power consumption of proposed algorithms comes out to be $0.45 \mathrm{~W}, 0.43 \mathrm{~W}, 0.31 \mathrm{~W}$ and $0.31 \mathrm{~W}$ for CSAIM, BCSBM, GMP and BMPNILS respectively which is significantly less than power consumed $(0.9 \mathrm{~W})$ if we scan the panel without using compressive sampling using the formula $P=N^{2} E F$. 


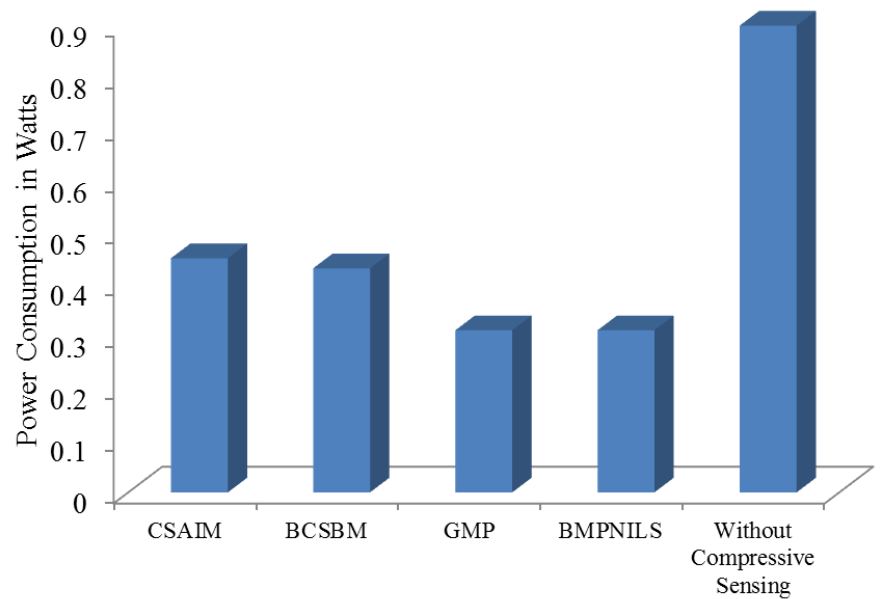

Figure 4.19: Power consumption comparison for CSAIM, BCSBM, GMP and BMPNILS.

\subsubsection{Implementation Analysis}

All state of the art greedy algorithms need least squares calculations, which brings in heavy computation cost. As indicated in [83], Richardson's iteration is proposed to solve the least squares problem, however the performance of Richardson's iteration depends upon the design of sampling matrix and can slow down the iteration convergence significantly. Also Richardson's iterations introduces an unnecessary overhead which can result in high latency when implemented in an embedded system.

The second issue is the dynamically changing dimensions of least squares operation in CoSaMP based algorithms. As an example, during the merging step in CoSaMP in [83], new atoms are added dynamically to the subset of sampling matrix which is then used in the least squares step. thus requiring dynamic memory allocation, which is not advisable according to the MISRA-C standard [119].

On the other hand, the algorithms proposed in this paper do not rely on least squares to recover a sparse signal, except for single instance at the end of BMPNILS. As a result, the number of multiplication operations required are reduced, making the algorithms faster and easier to implement.

Below, we analyze the algorithms in terms of number of multiplications required. 


\subsubsection{CSAIM}

When using a measurement vector of size $M \times 1$, in the worst case scenario, the number of operations (scalar comparisons and subtraction) would be $7 M$, and in the best case scenario, $3 M$. This is a huge improvement from the traditional greedy compressive sensing algorithms, as CSAIM does not require any matrix vector multiplication.

\subsubsection{BCSBM}

This algorithm requires a few matrix vector multiplications and some assignment operations. We will focus on the multiplication operations only. For a sampling matrix of size $M \times N$ and a measurement vector of $M$, the total number of multiplication operations required by the algorithm will be $(M+s k) N$, where $s$ is the block size and $k$ is the block-sparsity of the signal.

\subsubsection{GMP}

The Gramian matching pursuit removes the matrix vector multiplication in each iteration by exploiting the structure of the Hadamard matrix, and thus we are able to reduce number of multiplication operations to $M N+(J-1)(U+1+M)$, where $J$ is the number of iterations and $U$ is the number of unique values in the Gramian matrix. Line 14 costs only 1 multiplication. Line 7 when realized using a fast WalshHadamard transform (FWHT) [111], reduces number of multiplications from $M N$ to $N \log N$. Use of FWHT also ensures that we do not need to store sampling matrix. Thus the total number of multiplications for GMP are $N \log N+(J-1)(U+1+M)$. There is no pseudo-inverse calculation as well as no sorting operation in GMP which helps in saving resources. 
Table 4.1: Proposed algorithms comparison

\begin{tabular}{rrrr}
\hline Algorithm & $\begin{array}{r}\text { Exact sparsity } \\
\text { value required }\end{array}$ & Multiplications & $\begin{array}{c}\text { Minimum number of } \\
\text { measurements required }\end{array}$ \\
\hline CSAIM & No & 0 & $\frac{N}{2}+1$ \\
BCSBM & Yes & $(M+s k) N$ & $\frac{N}{s}+5 s k$ \\
GMP & No & $N \log N+(J-1)(U+1+M)$ & $f k \log N$ \\
BMPNILS & No & $2 J\left(N \log _{2} N\right)+M k+2 N \log _{2} N+J M$ & $f k \log N$ \\
\hline
\end{tabular}

\subsubsection{BMPNILS}

The first step in BMPNILS is the inner matrix product which takes $M N$ multiplications. The residual update and norm calculation takes another $M N+M$ multiplications. The least squares step at the end takes $M k+2 N \log _{2} N$ multiplications. The total number comes out to be $2 J\left(N \log _{2} N\right)+M k+2 N \log _{2} N+J M$ (not taking into account the pre-recovery steps), where $J$ is the total number of iterations. The least squares operation at the end can be realized by incorporating a fast FFT algorithm in order to reduce the number of multiplications. Table 4.1 summarizes the above discussed implementation comparison between these algorithms.

\subsubsection{Accumulated Stack Size}

It is known that number of FLOPS required by an algorithm has a direct effect on the touch registration. The static implementation stack size required by the algorithm is crucial in choosing because of low memory touch controllers being produced by industry. As seen below in Table 4.2, different algorithms have very different accumulated stack size when implemented in memory of touch controller. As a comparison, Block-CoSaMP has a stack size of $410 \mathrm{kB}$, which is double than BMPNILS.

\subsection{Validation of Algorithm Results}

Perfecting a sensor design is just the first step. As the capacitive touch panel size increases, sampling time also increases resulting in: 
Table 4.2: Accumulated Stack Size per Algorithm in kilo Bytes

\begin{tabular}{|l|r|}
\hline Algorithm & Accumulated Stack Size (kB) \\
\hline CSAIM & 0.1 \\
\hline BSCBM & 7.6 \\
\hline GMP & 2.6 \\
\hline BMPNILS & $\mathbf{2 0 9}$ \\
\hline Block-CoSaMP & 410 \\
\hline
\end{tabular}

1. Low Sampling Rate

2. Delay in Touch Registration

3. Bad User Experience

This problem can be treated as an academic research problem. The solution is to design new efficient and accurate sampling techniques. In this chapter, structured and random sampling matrices are used in an attempt to propose efficient touch location recovery algorithms. The algorithms proposed in this chapter were designed to be more accurate and efficient than state-of-the-art algorithms in capacitive touch sampling $[83,114]$. It can be seen in Figure 4.14 and Figure 4.17, that the proposed algorithms are more accurate and efficient than the already published state-of-the-art in this field. Not only that, the algorithms proposed in this thesis are more efficient than the state-of-the-art algorithms for block sparse sensing literature $[115,116]$ and with comparable accuracy (see Figure 4.15 and 4.18). An important point to note here is that although our proposed algorithm BMPNILS was initially designed to solve sampling problem associated with large-scale touch panels, it can be utilized in other signal processing applications as well now that it has proved itself to be of higher efficiency and accuracy. The results have also been published in IEEE proceedings [30]. 


\subsection{Conclusion and Future Work}

The main objective of this chapter is to provide efficient and accurate sampling algorithms for large-scale capacitive touch panels. The objective is to minimize the sampling time, reduce power consumption and increase accuracy for large-scale touch panels. Compressive sensing when used with capacitive touch technology can help achieve these objectives. We designed two binary structured matrices which can be used as driving voltage values for touch panel. We also investigated greedy algorithms and looked at unique characteristics of capacitive touch signal in order to propose an accurate and efficient variation of block matching pursuit. We proposed simple and easy to implement recovery algorithms by exploiting the structure of the sensing matrix, and conducted various experiments including recovery and FLOPS analysis to compare and contrast our algorithms and see how they stack against other greedy algorithms. As observed from the simulations, BMPNILS has comparable accuracy with other greedy algorithms whereas CSAIM is the most efficient recovery algorithm. Based on the requirements, the panel designers can employ one of the four recovery proposed algorithms for their capacitive touch panels. 


\section{Chapter 5}

\section{Subpixel Interpolation}

\subsection{Introduction}

In the previous two chapters, we discussed sensor pattern design and efficient sampling algorithms for capacitive touch sensing. Another important part of the overall capacitive touch system is subpixel interpolation. After sampling, it is important for the touch controller to deploy subpixel interpolation algorithms for an accurate finger location detection. Center Of Mass (COM) interpolator was used in Chapter 3 in order to test various sensor patterns. In this chapter, multiple subpixel interpolation algorithms will be studied in detail and simulations will be performed to find out an accurate interpolation technique for touch panels.

It has been mentioned before that the diameter of the sensor capacitor is assumed to be smaller than the diameter of a finger. Baharav and Kakarala [58] derived an analytic solution for a wire grid sensor pattern shown in Figure 5.1. They assumed that the wire pitch is narrower than diameter of a normal sized finger and that the finger can be modeled as a circle. It can be stated that the response of the sensor is proportional to the area covered by the round finger.

In Figure 5.1, if we denote capacitance value at $C_{2}$ and $C_{3}$ as $c_{2}$ and $c_{3}$, we realize that $c_{2}$ and $c_{3}$ are just chords of the finger circle. Using basic geometric equations, 


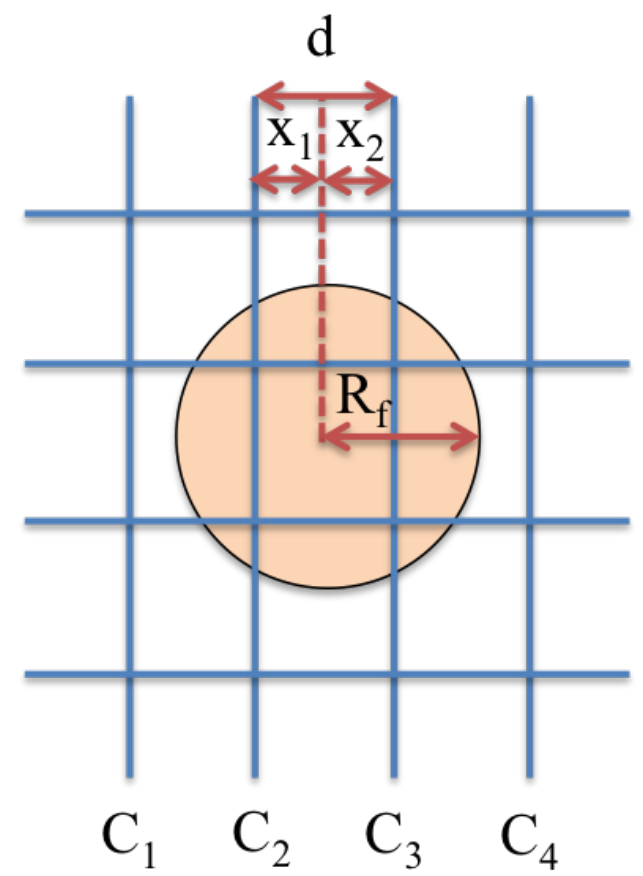

Figure 5.1: Wire grid sensor pattern with finger modeled as a 2-D circle

we can find the true center of the finger that lies between $C_{2}$ and $C_{3}$.

$$
\begin{gathered}
x_{1}=\sqrt{R_{f}^{2}-\left(\frac{c_{2}}{2}\right)^{2}} \\
x_{2}=\sqrt{R_{f}^{2}-\left(\frac{c_{3}}{2}\right)^{2}} \\
d=x_{1}+x_{2}=\sqrt{R_{f}^{2}-\left(\frac{c_{2}}{2}\right)^{2}}+\sqrt{R_{f}^{2}-\left(\frac{c_{3}}{2}\right)^{2}}
\end{gathered}
$$

Such analytical solutions can work for simple wire grid designs. However as it was seen in Chapter 3, sensor design can have complicated geometry and in order to find out the accurate finger position, controller has to resolve to subpixel interpolation. Capacitive touch panel is treated as an image processing problem in this thesis. If finger is treated as a bell shaped blob, it can be observed that the blob may occupy more than one pixel as shown in Figure 5.2. In this chapter, the term 1 pixel refers to a single capacitor sensor on the grid. 


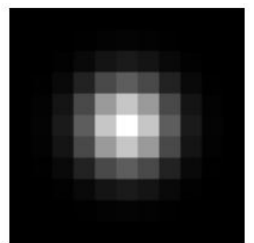

Figure 5.2: A zoomed in image of finger response on the panel. Notice that a single finger covers many pixels.

An approximate model of finger response can be a 2-D Gaussian point as its edges are smooth and blurred. Looking back at $(4.35),(u, v)$ can give the peak location of the point. Normal interpolation algorithms can estimate values up to single pixel accuracy. However, in order to estimate the position of finger, pixel size accuracy will not give accurate results as the values are in continuous range with subpixel precision. Thus subpixel interpolation algorithms are required.

Fisher and Naidu [10] compared four such subpixel interpolation algorithms namely Gaussian, COM, linear and parabolic while determining the position of a light stripe with subpixel accuracy. The estimators (or interpolators) are listed in table 5.1. It is important to note here that in the process of estimating finger location, the interpolation algorithms will estimate both row and column readings separately and it is the job of the processor to combine the two estimates. Thus, let us call them $2 \times 1$-D interpolators or simply three point interpolators. It is assumed here for the sake of simplicity that the finger will occupy more than one basic element of row $\left(r_{i}\right)$ and column $\left(c_{i}\right)$. The maximum value of the row reading is denoted by $r_{i}$ whereas $r_{i-1}$ and $r_{i+1}$ indicate the reading in the row below and above the maximum respectfully. For each interpolator, three values will be used to calculate estimated row or column coordinates as seen in Table 5.1. For each interpolator, the $r$ values are assumed to be non-negative, and in the case of Gaussian estimator, positive.

Gaussian and COM estimators assume that the observed peak shape of the readings will follow a Gaussian profile. The linear estimator on the other hand assumes a linear relationship between the spread of values before and after the peak reading. Similarly, a parabolic interpolator will try to fit the signal using a parabolic curve 
Table 5.1: Three-point subpixel interpolation algorithms. Formulas taken from [10].

\begin{tabular}{|c|c|}
\hline Interpolator & Formula \\
\hline Center of Mass & $\frac{r_{i+1}-r_{i-1}}{r_{i-1}+r_{i}+r_{i+1}}$ \\
\hline Gaussian & $\frac{1}{2} \frac{\ln r_{i-1}-\ln r_{i+1}}{\ln r_{i-1}-2 \ln r_{i}+\ln r_{i+1}}$ \\
\hline Linear & $\frac{1}{2} \frac{r_{i+1}-r_{i-1}}{r_{i}-\min \left[r_{i-1}, r_{i+1}\right]}$ \\
\hline Parabolic & $\frac{1}{2} \frac{r_{i-1}-r_{i+1}}{r_{i-1}-2 r_{i}+r_{i+1}}$ \\
\hline
\end{tabular}

[10]. It can be verified that the subpixel estimate value generated by these estimators lies between -0.5 to $0.5[58]$.

\subsection{Resolution of Capacitive Touch Panels}

The smallest change in finger or stylus movement across the panel that can be detected by the touch controller is labeled as touch panel resolution. For capacitive touch panels, the coarse-resolution of the panel is the physical distance between the sensors embedded on it. This distance is sometimes titled as pitch. As an example, the coarse-resolution is equal to $5 \mathrm{~mm}$ if the sensors are spaced $5 \mathrm{~mm}$ apart. However, the resolution achieved on the sensor level is not satisfactory for most touch based applications. Figure 5.3 shows example capacitance readings from sensors $\mathrm{x} 0, \mathrm{x} 1$ and $\mathrm{x} 2$. Using coarse-resolution, the finger location will be identified as the location of sensor $\mathrm{x} 1$, however as it can be seen, the true peak location will lie somewhere between location of $\mathrm{x} 1$ and $\mathrm{x} 2$. It is not feasible to increase the number of sensors for large-scale panels in order to increase resolution, because of 
manufacturing issues and increase in scanning time [82]. The coarse-resolution can only be increased by performing interpolation between sensor readings in order to get accurate finger touch coordinates. There are two basic steps for interpolation:

1. The host controller finds the coarse-resolution peak of finger reading after signal recovery and noise filtering.

2. The host controller then has to use one of many available interpolation algorithms to find the fine location or super-resolution between sensor readings.

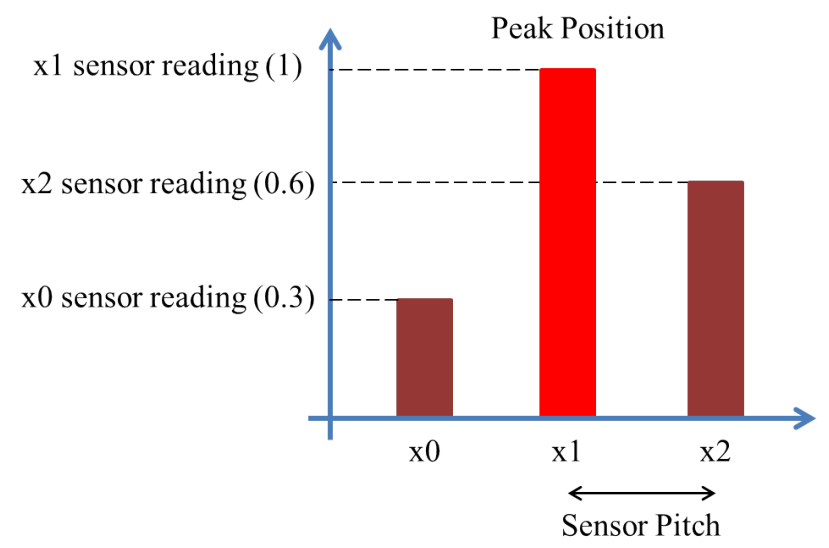

Figure 5.3: Figure depicting sensor readings and coarse peak position.

Figure 5.4 shows the three point linear interpolation result on example sensor readings. The interpolated peak location falls in between $\mathrm{x} 1$ and $\mathrm{x} 2$ sensor location.

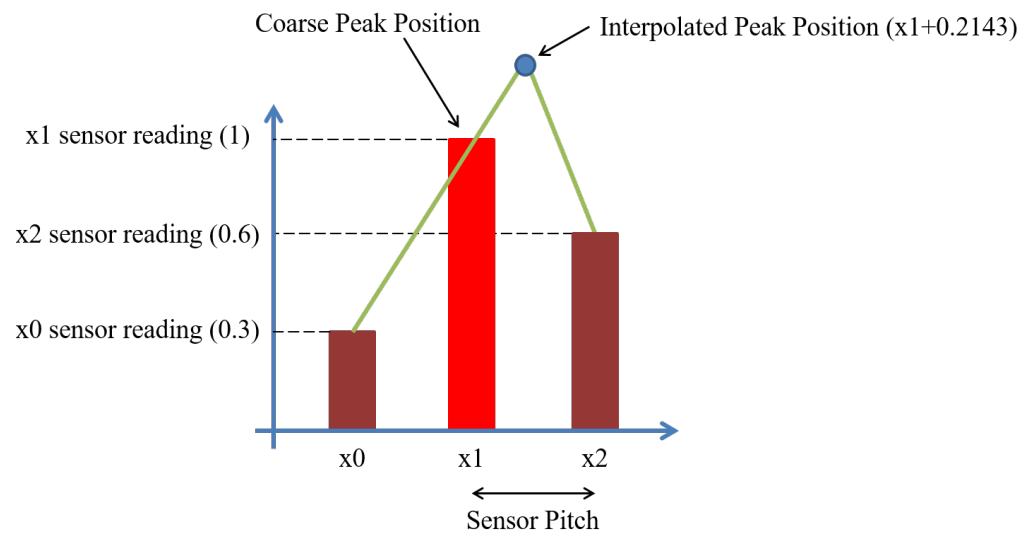

Figure 5.4: Figure depicting peak position derived by subpixel linear interpolation. 
As an example, in Figure 5.4, the interpolated touch position will be the location of $\mathrm{x} 1$ plus 0.2143 (the result from linear interpolator formula). The sensor resolution can be increased theoretically by thinking of capacitive sensors as pixelated binary images. In Chapter 3, grid patterns were designed as binary images with each sensor having size of $100 \times 100$ pixels. This means that within each sensor, we can have 100 different possible locations for $\mathrm{x} 1$ sensor, or in other words, the resolution is equal to 100. For example, a diamond sensor panel with 10 sensors will have a total resolution of $100 \times(10-1)=900$ points, as shown in Figure 5.5. This resolution is theoretical and can be increased or decreased. It can be set in practical applications by performing extensive sensitivity tests on a touch-panel grid using a robotic finger placed at multiple locations on a single sensor.

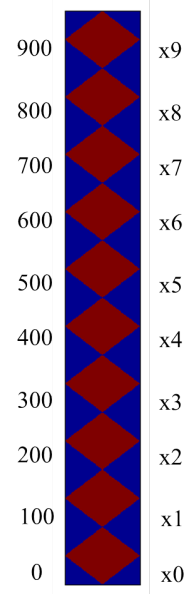

Figure 5.5: An example diamond pattern column showing fine resolution values for each sensor $\mathrm{x} 0-\mathrm{x} 9$.

\subsubsection{Finger Modeling for Capacitive Touch Panels}

Up to this point, capacitive touch signal has been modeled as a 2-D Gaussian function. This assumption was based on the shape of the finger in contact with the panel and also because generally, academics in related fields tend to use 2-D Gaussian fingers in their research $[58,65,66,67]$. 
A sophisticated finger model would be the one which incorporates pitch angle of the finger when touching the screen. Pitch angle is defined as the angle between finger and the cover glass of the panel. Individual users may touch the panel with different pitch angles. A finger resting on the panel with varying pitch angle will generate a varying response as shown in Figure 5.6. Existing literature utilizes the pitch angle info to generate more accurate finger models [120, 121], but to the best of our knowledge, the connection of pitch angle with subpixel interpolation is not yet investigated. In this chapter, we will look at different interpolation algorithms and simulate them for varying pitch angle responses.

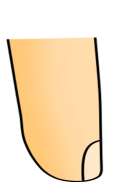

(a) $90^{\circ}$

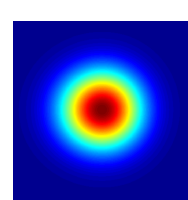

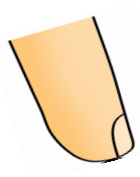

(b) $75^{\circ}$

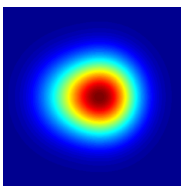

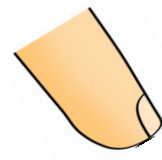

(c) $60^{\circ}$

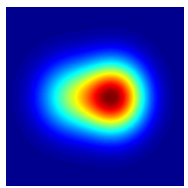

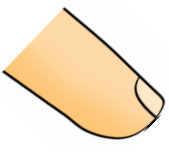

(d) $45^{\circ}$

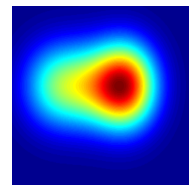

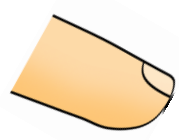

(e) $30^{\circ}$

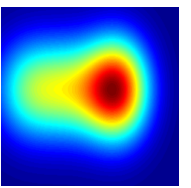

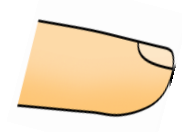

(f) $15^{\circ}$

Figure 5.6: Varying pitch angle of the finger while the position of the touch panel contact remains unchanged.

In upcoming sections, accuracy of various subpixel interpolators will be tested with varying finger pitch angles.

\subsection{One Dimensional Three-Point Interpolators}

From equations in Table 5.1, it is not obvious which algorithm will perform better in capacitive touch panel environment. There is no explanation available of why touch panel manufacturing industry would opt for one interpolation algorithm over the other. Lack of existing theoretical work linking subpixel interpolators with capacitive touch panels is an exciting and motivating factor for this research. To the best of our knowledge, the only published instance of linear interpolator being 
used in capacitive touch screens in industry was in a white paper by Microchip [122], where a three-point linear interpolator was used.

Previously, in Chapter 3, diagonal, circular and angle sweep movement were performed on different sensor patterns using COM interpolator. The grid pattern test methodology was designed for self capacitance systems. It was briefly hinted that the methodology can be adapted for mutual capacitance but the details were not given. We will explain below how COM interpolator can be used for both self and mutual capacitance schemes.

Consider a 2-D Gaussian finger resting on a touch panel shown in Figure 5.7 at a $90^{\circ}$ pitch angle. If the touch sensing scheme is self-capacitance, then the host controller will recover the sum of sensors (c1 to c9) for every sensor column under the finger area and the sum of sensors for each row under the finger area (r1 to r12). It will then send six readings (3 row-sum readings and 3 column-sum readings) to COM in order to find the subpixel peak location.

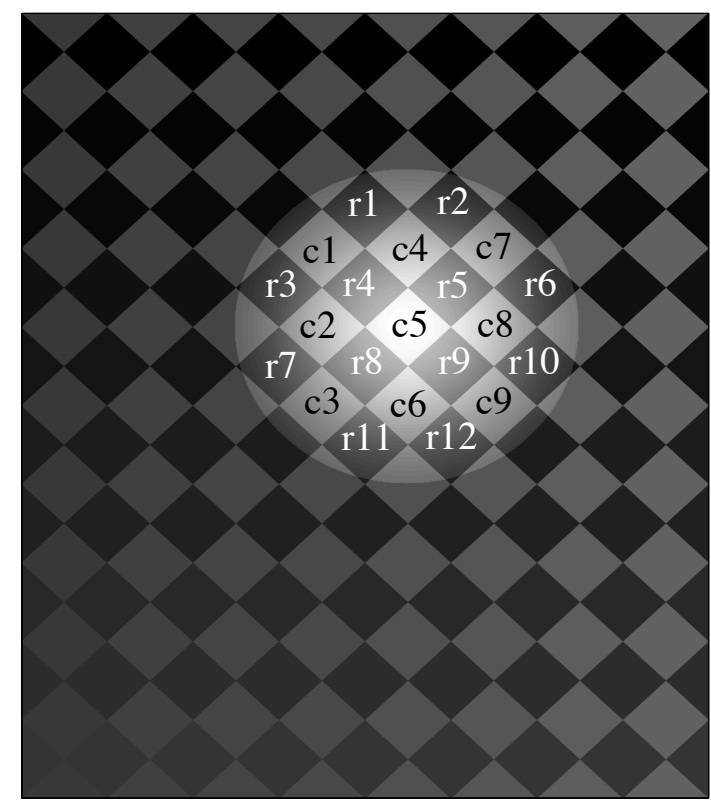

Figure 5.7: Finger superimposed on a diamond grid. r1-r12 and c1-c9 depict the number of sensors triggered due to finger presence. 
In the case of mutual capacitance, the host controller will recover the individual readings of sensors ( $\mathrm{c} 1$ to $\mathrm{c} 9$ and $\mathrm{r} 1$ to $\mathrm{r} 12$ ) under the finger area using traditional sampling or using one of the four proposed algorithms in Chapter 4. It can then, either

1. send the six maximum individual sensor readings to COM or,

2. calculate the row and column sum over the panel and send the summed values to the interpolator.

Both approaches will guarantee subpixel accuracy, but the second approach will result in more accurate results because more sensor readings will be used by COM. In either case, it is a straightforward procedure to use three-point interpolators for self or mutual capacitance sensing schemes.

In the current section, first, we would re-run simulations for grid pattern test methodology using the four interpolators given in Table 5.1 and compare the subpixel accuracy results for a 2-D Gaussian finger with varying pitch angles. We will also present a simple theoretical explanation of the results.

\subsubsection{Diagonal Movement}

Similar to the experiment in Chapter 3, finger was placed at a known location on interleaved sensor pattern and dragged towards the center of the panel in a straight line at a $45^{\circ}$ angle. Mean error in millimeters was calculated by finding the Euclidean distance between the true and estimated location in each iteration and then by taking the average of resulting vector at the end of the diagonal motion. The experiment was repeated for different pitch angles. Figure 5.8 shows the tracking results for a finger moving on interleaved pattern in a diagonal motion at $90^{\circ}$ pitch angle.

Table 5.2 shows the mean tracking error for diagonal movement. There are a couple of interesting insights that can be acquired from the table. 


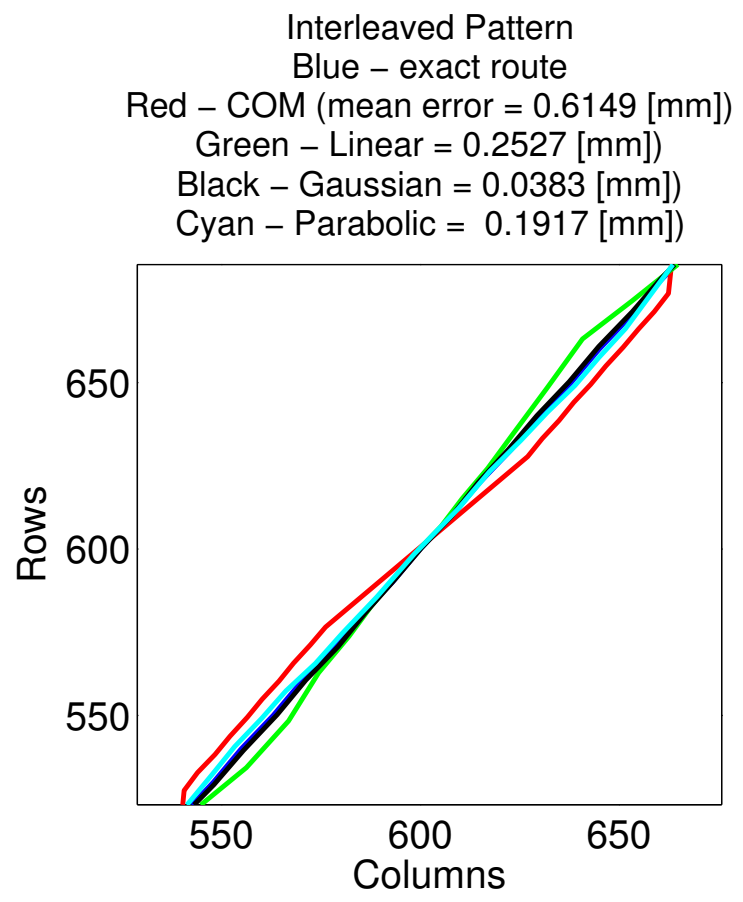

Figure 5.8: Finger of radius $=7.5 \mathrm{~mm}$ moving in a diagonal path across the interleaved grid touch panel (zoomed in, without noise). The units for rows and columns are in pixels (100 pixels $=5 \mathrm{~mm})$. Figure is best viewed in color.

1. Gaussian interpolator is by far the most accurate one in the case of noiseless and noisy measurements.

2. When the pitch angle is very small, the difference in performance between Gaussian and other interpolators becomes small too. We have also added random multiplicative noise with amplitude 0.3 to the finger measurements. At very small pitch angles, the shape of touch response gets very skewed and results in a large subpixel error.

3. When the pitch angle is $90^{\circ}$, it is recommended to use Gaussian interpolator because of its superior performance. For wall displays, it is typical for the user to touch the panel with a pitch angle of $90^{\circ}$.

These results are based on simulation and in order to back them up, we performed theoretical analysis for the Gaussian interpolator to show that it is ideal for our application. The cross section of the finger for $90^{\circ}$ pitch angle can be represented 
Table 5.2: Interleaved pattern diagonal movement tracking error comparison for varying pitch angle. Smallest error value is shown in bold.

Mean tracking error $(\mathrm{mm})$ for varying pitch

\begin{tabular}{ccccccc}
\hline \multicolumn{7}{c}{ Finger radius $=7.5 \mathrm{~mm}$ with zero noise } \\
\hline Interpolator & $90^{\circ}$ & $75^{\circ}$ & $60^{\circ}$ & $45^{\circ}$ & $30^{\circ}$ & $15^{\circ}$ \\
COM & 0.6149 & 1.018 & 1.8063 & 2.0823 & 2.1106 & 2.1128 \\
Linear & 0.2527 & 0.9839 & 1.8059 & 2.1707 & 2.2383 & 2.2434 \\
Gaussian & $\mathbf{0 . 0 3 8 3}$ & $\mathbf{0 . 7 3 6 5}$ & $\mathbf{1 . 5 3 7 4}$ & $\mathbf{1 . 8 8 1 8}$ & $\mathbf{1 . 9 4 3 1}$ & $\mathbf{1 . 9 4 7 5}$ \\
Parabolic & 0.1917 & 0.9632 & 1.7656 & 2.1071 & 2.1669 & 2.1712
\end{tabular}

\begin{tabular}{ccccccc}
\hline \multicolumn{6}{c}{ Finger radius $=7.5 \mathrm{~mm}$ with multiplicative noise of amplitude 0.3} \\
\hline Interpolator & $90^{\circ}$ & $75^{\circ}$ & $60^{\circ}$ & $45^{\circ}$ & $30^{\circ}$ & $15^{\circ}$ \\
COM & 0.9716 & 1.3479 & 2.1229 & 2.2944 & 2.391 & 2.457 \\
Linear & 0.62 & 1.2557 & 2.0287 & 2.1739 & 2.3609 & 2.3815 \\
Gaussian & $\mathbf{0 . 5 8 4 8}$ & $\mathbf{1 . 1 9 4 8}$ & $\mathbf{1 . 9 6 8 4}$ & $\mathbf{1 . 9 6 2 8}$ & $\mathbf{2 . 3 0 5 7}$ & $\mathbf{2 . 3 4 5 7}$ \\
Parabolic & 0.6933 & 1.2872 & 2.0527 & 2.1026 & $\mathbf{2 . 2 9 6 9}$ & $\mathbf{2 . 3 4 6 9}$
\end{tabular}

\begin{tabular}{ccccccc}
\hline \multicolumn{7}{c}{ Finger radius $=5 \mathrm{~mm}$ with zero noise } \\
\hline Interpolator & $90^{\circ}$ & $75^{\circ}$ & $60^{\circ}$ & $45^{\circ}$ & $30^{\circ}$ & $15^{\circ}$ \\
COM & 1.0268 & 1.3965 & 1.9213 & 2.0009 & 2.0029 & 2.0304 \\
Linear & 0.0821 & 0.9259 & 1.4256 & 1.495 & 1.4966 & 1.5015 \\
Gaussian & $\mathbf{0 . 0 0 9 1}$ & $\mathbf{0 . 8 7 8 9}$ & $\mathbf{1 . 3 7 2 6}$ & $\mathbf{1 . 4 2 5}$ & $\mathbf{1 . 4 3 2}$ & $\mathbf{1 . 4 4 1 3}$ \\
Parabolic & 0.3993 & 0.9452 & 1.3762 & 1.4333 & 1.4384 & 1.4454
\end{tabular}

by a one dimensional Gaussian function:

$$
f(x)=\exp \left[-\frac{(x-u)^{2}}{2 \sigma^{2}}\right]
$$

where $u$ is the real offset and $\sigma$ is the standard deviation. To calculate the estimated offset $\hat{u}$, we substitute $f(x)$ in Gaussian interpolator function for $x=-1,0$ and 1 .

$$
\begin{aligned}
\hat{u} & =\frac{1}{2}\left(\frac{\ln f(-1)-\ln f(1)}{\ln f(-1)-2 \ln f(0)+\ln f(1)}\right) \\
& =\frac{1}{2}\left(\frac{\ln \left(\exp \left[-\frac{(-1-u)^{2}}{2 \sigma^{2}}\right]\right)-\ln \left(\exp \left[-\frac{(1-u)^{2}}{2 \sigma^{2}}\right]\right)}{\ln \left(\exp \left[-\frac{(-1-u)^{2}}{2 \sigma^{2}}\right]\right)-2 \ln \left(\exp \left[-\frac{(-u)^{2}}{2 \sigma^{2}}\right]\right)+\ln \left(\exp \left[-\frac{(1-u)^{2}}{2 \sigma^{2}}\right]\right)}\right)
\end{aligned}
$$




$$
\begin{aligned}
& =\frac{1}{2}\left(\frac{-(-1-u)^{2}+(1-u)^{2}}{-(-1-u)^{2}+2(u)^{2}-(1-u)^{2}}\right) \\
& =\frac{1}{2}\left(\frac{-1-u^{2}-2 u+1+u^{2}-2 u}{-1-u^{2}-2 u-1-u^{2}+2 u+2 u^{2}}\right) \\
& =\frac{1}{2}\left(\frac{-4 u}{-2}\right) \\
& =u .
\end{aligned}
$$

For COM, the first order Taylor series expansion for (5.4) will be:

$$
\hat{f(x)}=\left(1+\frac{x u}{\sigma^{2}}\right) \exp \left[-\frac{x^{2}}{2 \sigma^{2}}\right]
$$

Substituting (5.11) into COM formula for $x=-1,0$ and 1 , we get:

$$
\begin{aligned}
\hat{u} & =\frac{f \hat{(1)}-f(\hat{-}-1)}{f(\hat{-}-1)+\hat{f(0)}+\hat{f(1)}} \\
& =\frac{\left(1+\frac{u}{\sigma^{2}}\right) \exp \left[\frac{-1}{2 \sigma^{2}}\right]-\left(1-\frac{u}{\sigma^{2}}\right) \exp \left[\frac{-1}{2 \sigma^{2}}\right]}{\left(1-\frac{u}{\sigma^{2}}\right) \exp \left[\frac{-1}{2 \sigma^{2}}\right]+1+\left(1+\frac{u}{\sigma^{2}}\right) \exp \left[\frac{-1}{2 \sigma^{2}}\right]} \\
& =\frac{\left(\sigma^{2}+u\right) \exp \left[\frac{-1}{2 \sigma^{2}}\right]-\left(\sigma^{2}-u\right) \exp \left[\frac{-1}{2 \sigma^{2}}\right]}{\left(\sigma^{2}-u\right) \exp \left[\frac{-1}{2 \sigma^{2}}\right]+\sigma^{2}+\left(\sigma^{2}+u\right) \exp \left[\frac{-1}{2 \sigma^{2}}\right]} \\
& =\frac{2 u \exp \left[\frac{-1}{2 \sigma^{2}}\right]}{2 \sigma^{2} \exp \left[\frac{-1}{2 \sigma^{2}}\right]+\sigma^{2}} \\
& =u\left(\frac{2 \exp \left[\frac{-1}{2 \sigma^{2}}\right]}{\sigma^{2}+2 \sigma^{2} \exp \left[\frac{-1}{2 \sigma^{2}}\right]}\right) .
\end{aligned}
$$

Similarly, for linear and parabolic estimators, we get (5.17) and (5.18) respectively.

$$
\hat{u}=u\left(\frac{\exp \left[\frac{-1}{2 \sigma^{2}}\right]}{\sigma^{2}-\sigma^{2} \exp \left[\frac{-1}{2 \sigma^{2}}\right]}\right) .
$$




$$
\hat{u}=u\left(\frac{\exp \left[\frac{-1}{2 \sigma^{2}}\right]}{2 \sigma^{2}-2 \sigma^{2} \exp \left[\frac{-1}{2 \sigma^{2}}\right]}\right) .
$$

As an example, if $\sigma=1$ then,

- Bias for Gaussian: $\hat{u}=u$

- Bias for COM: $\hat{u}=0.54 u$

- Bias for linear: $\hat{u}=1.54 u$

- Bias for parabolic: $\hat{u}=0.77 u$

These results show that Gaussian estimator has the least bias in ideal conditions (perfect Gaussian input signal) followed by COM estimator. However, it will be shown later, COM estimator has an overall poorer performance than linear and parabolic estimator. Because COM is just a weighted average, it suffers from pixel or peak locking when the finger tip falls in the middle of two sensors. Gaussian interpolator, on the other hand is an ideal solution for large-scale wall displays where in most cases the finger pitch angle is approximately equal to 90 degrees.

\subsubsection{Circular Movement}

Figure 5.9 shows the tracking results for a finger moving on interleaved pattern in a circular motion at $90^{\circ}$ pitch angle.

Table 5.3 shows the interpolators performance for circular movement at different pitch angles. Overall, Gaussian interpolator gives the most accurate result. For an ideal Gaussian response, all four interpolators perform with acceptable error, whereas there is a noticeable increase in error with the decrease of pitch angle. For Gaussian interpolator, there is not much difference in performance between diagonal and circular motion (difference of $0.0042 \mathrm{~mm}$ ) as opposed to COM interpolator where the difference is of $0.29 \mathrm{~mm}$. 


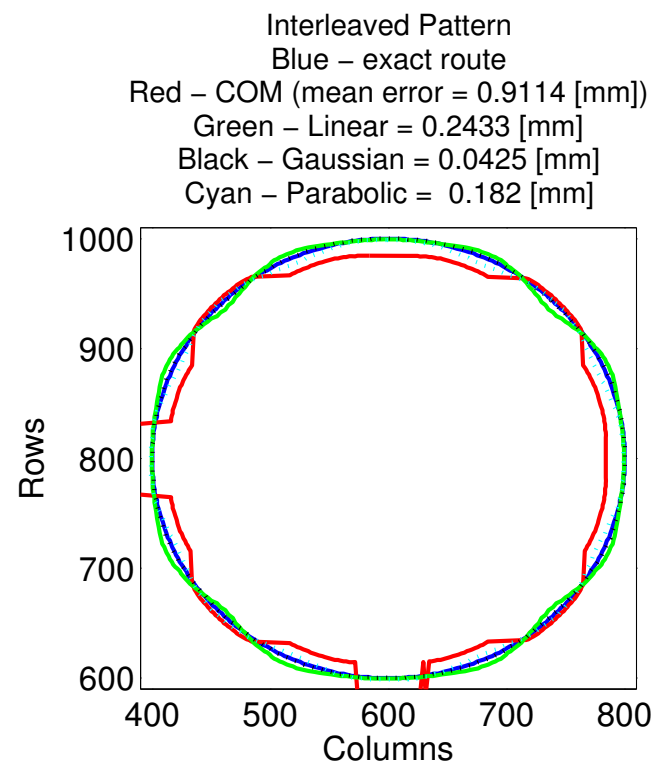

Figure 5.9: Finger of radius $=7.5 \mathrm{~mm}$ moving in a circular path across the interleaved grid touch panel (without noise). The units for rows and columns are in pixels (100 pixels $=5 \mathrm{~mm}$ ). Figure is best viewed in color.

Table 5.3: Interleaved pattern circular movement mean tracking error (mm) with finger radius $7.5 \mathrm{~mm}$.

\section{Mean tracking error $(\mathrm{mm})$ for varying pitch}

Finger radius $=7.5 \mathrm{~mm}$ with zero noise

$\begin{array}{ccccccc}\text { Interpolator } & 90^{\circ} & 75^{\circ} & 60^{\circ} & 45^{\circ} & 30^{\circ} & 15^{\circ} \\ \text { COM } & 0.9114 & 1.2735 & 1.7856 & 2.1008 & 2.1694 & 2.1746 \\ \text { Linear } & 0.2433 & 0.9369 & 1.7665 & 2.1755 & 2.2579 & 2.2644 \\ \text { Gaussian } & \mathbf{0 . 0 4 2 5} & \mathbf{0 . 9 0 1 3} & \mathbf{1 . 7 3 1 9} & \mathbf{2 . 0 3 6 1} & \mathbf{2 . 0 5 8 7} & \mathbf{2 . 0 6 0 4} \\ \text { Parabolic } & 0.182 & 0.9878 & 1.7923 & 2.1451 & 2.2102 & 2.215\end{array}$

While comparing Table 5.3 and 5.2, it can be inferred that there is an overall decrease in subpixel accuracy. As diagonal motion only corresponds to a $45^{\circ}$ motion along the panel, an overall increase in error means that the interpolators do not perform equally well with angles other than $45^{\circ}$. In order to further investigate this, we will perform an angle sweep across the panel in next section.

\subsubsection{Angle Sweep Movement}

In order to thoroughly investigate the interpolators performance, an angle sweep per every $10^{\circ}$ was performed on interleaved pattern to check the performance of 


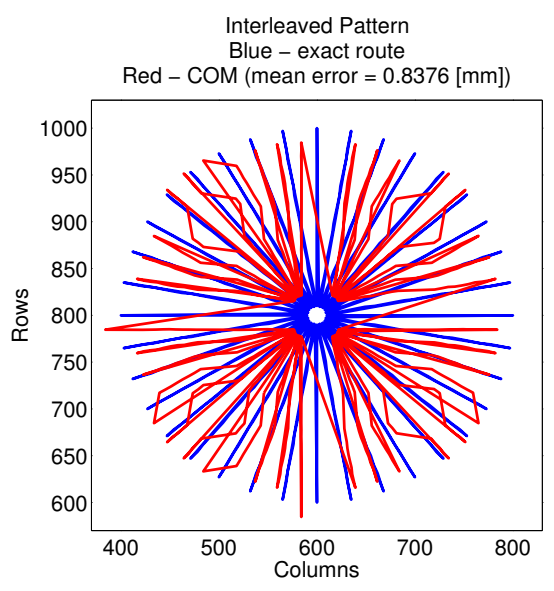

(a) $\mathrm{COM}$

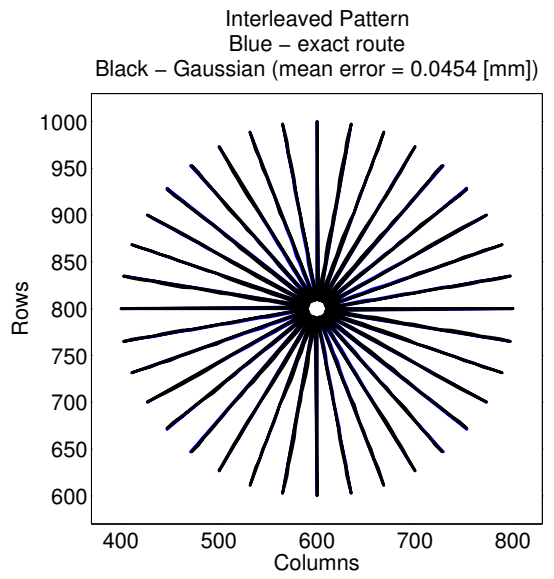

(c) Gaussian

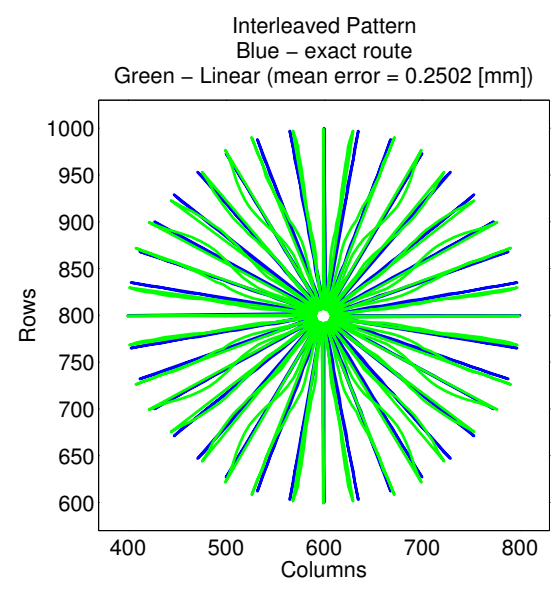

(b) Linear

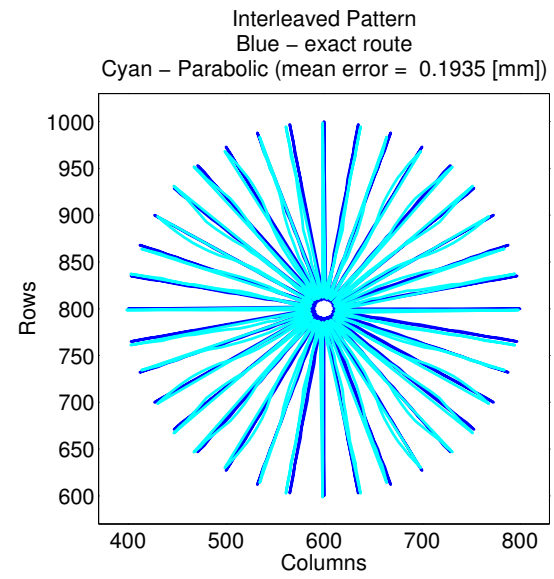

(d) Parabolic

Figure 5.10: Angle sweep performed by a $7.5 \mathrm{~mm}$ radius finger for different interpolators. The units for rows and columns are in pixels (100 pixels $=5 \mathrm{~mm})$. Figure is best viewed in color.

estimators in terms of deviation from the exact (or true) route. The results are presented in Table 5.4. Once more, it can be concluded that the Gaussian interpolator for interleaved pattern gives the most accurate results and performs equally well for all angles around the panel. Figure 5.10 shows the tracking results with four interpolators for a finger moving on interleaved pattern at $90^{\circ}$ pitch angle. It can be seen that the error increases considerably for horizontal and vertical motion along the panel, indicating presence of bias, especially for COM estimator. 
Table 5.4: Interleaved pattern angle sweep mean tracking error ( $\mathrm{mm})$ with finger radius $7.5 \mathrm{~mm}$.

\begin{tabular}{ccccccc}
\multicolumn{8}{c}{ Mean tracking error $(\mathbf{m m})$ for varying pitch } \\
Interpolator & $90^{\circ}$ & $75^{\circ}$ & $60^{\circ}$ & $45^{\circ}$ & $30^{\circ}$ & $15^{\circ}$ \\
COM & 0.8376 & 1.1978 & 1.7891 & 2.1521 & 2.2385 & 2.2404 \\
Linear & 0.2502 & 0.9622 & 1.7328 & 2.1714 & 2.2443 & 2.2499 \\
Gaussian & $\mathbf{0 . 0 4 5 4}$ & $\mathbf{0 . 9 0 5 5}$ & $\mathbf{1 . 7 2 6 3}$ & $\mathbf{2 . 0 8 2 1}$ & $\mathbf{2 . 1 4 6 6}$ & $\mathbf{2 . 1 5 1 4}$ \\
Parabolic & 0.1935 & 0.9754 & 1.7777 & 2.123 & 2.1847 & 2.1892
\end{tabular}

\subsubsection{Horizontal, Vertical Movement and Bias of Estimators}

If the finger is moved vertically or horizontally in a straight line, it is evident upon careful observation that there is a bias in the readings (see Figure 5.11).

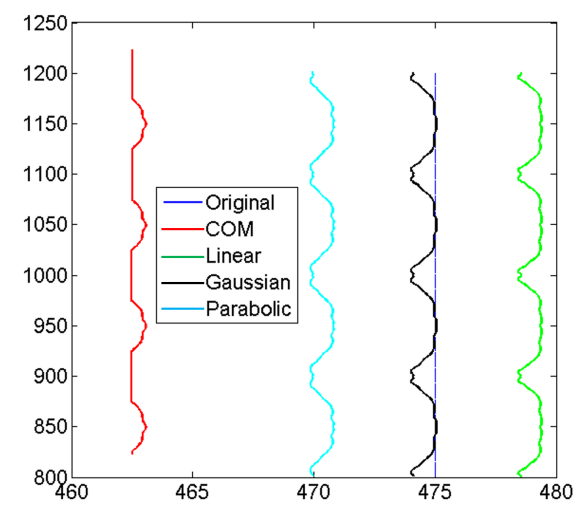

Figure 5.11: Bias in estimator result due to the same column value input to the estimators throughout the motion of the finger in the interleaved pattern. Notice that the horizontal axis is scaled to show the bias. Units for rows and columns are in pixels. (100 pixels $=5 \mathrm{~mm}$ ). Figure is best viewed in color.

This kind of bias was first observed in Chapter 3 for COM interpolator. The error induced in the position estimate is due to the mechanism of how the interpolation algorithms work. If the position of the finger with respect to the columns is not changed by the user throughout the motion, the fitting algorithms will have same column reading throughout the simulation while tracking the finger position. This can result in a non-uniform fitting curve with a shifted global maxima thus adding a constant bias to the estimate. This type of error is different from the tracking 
error due to random noise as this only induces bias in an estimate and can be easily removed from the final result.

To see the effect of bias in simulations, the finger starting column position was changed from 450 to 750 pixels in steps of 10 to generate a graph (Figure 5.12) showing the trend of the bias for all the estimators. The value of bias is obtained by subtracting the mean of the estimator result from the true value.
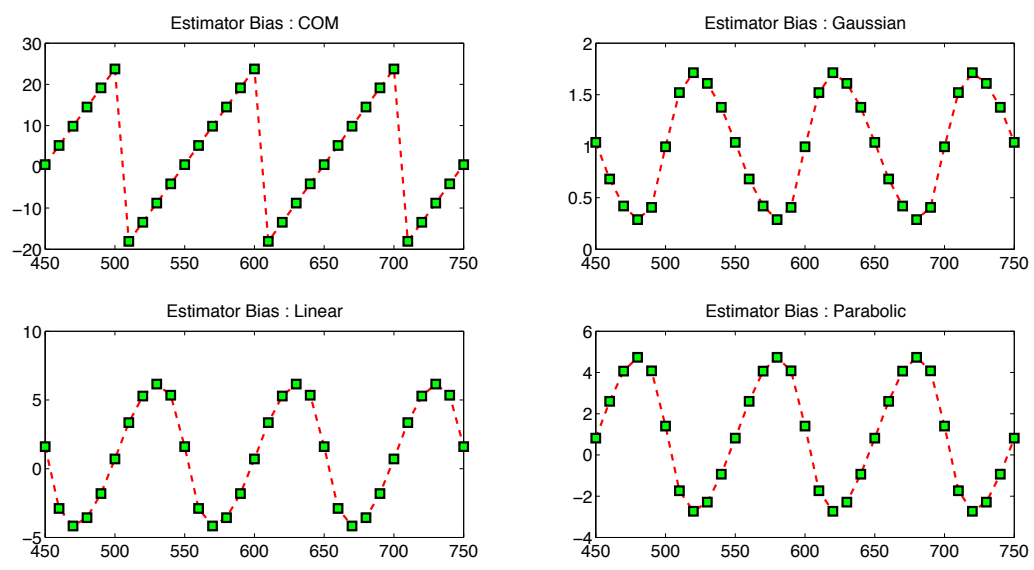

Figure 5.12: Bias in subpixel estimators. The column value was changed from 450 to 750 in steps of 10 after a single vertical motion by the finger and then mean of the estimator result was subtracted from the true value to get the bias. Notice that COM estimator has poor performance when compared with other estimators.

\subsubsection{Edge Effect}

As the finger reaches the edge of the panel, the performance of the interpolation algorithms get worse even with no additional noise. This is because of the lack of row and column readings available at the edges of the screen for the interpolation algorithms to perform well. As each algorithm requires minimum three readings (see Table 5.1) to calculate an estimate; at the edge, the readings reduce to two and then finally to one as the finger continues to move outside the panel. The missing readings if treated as zeros make the results inaccurate. 
In order to reduce the edge effect, a check was imposed, so that, whenever finger reached the edge and there were only two values $(A$ and $B$ ) available instead of three, we switched to a simpler two point linear algorithm where $X$ varies from 0 to 1 . The value calculated by this algorithm were used to get a better estimate of the finger movement at the edge. The results are shown in Figure 5.13.

$$
X=1-(0.5) *(\min (A, B) / \max (A, B))
$$

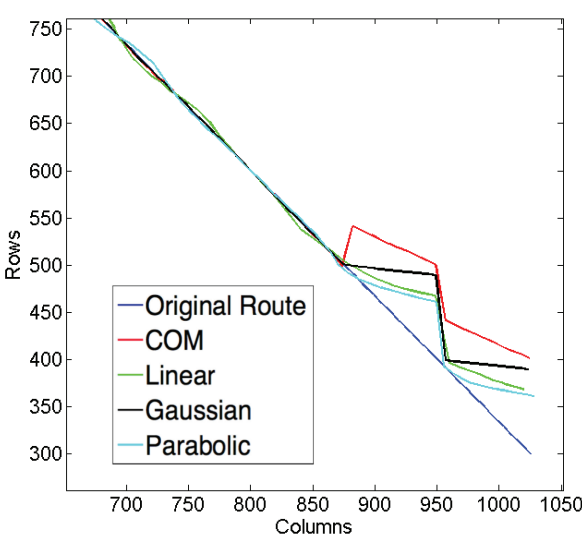

(a)

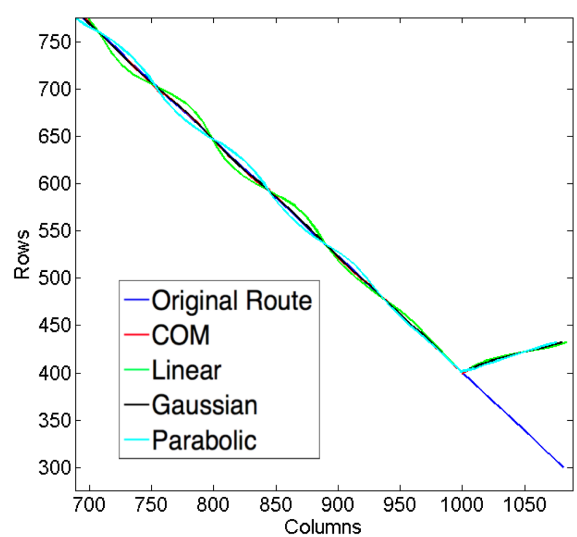

(b)

Figure 5.13: Minimizing the edge effect on interleaved grid. (a) Original result. Notice that due to the finger diameter being 300 pixels, the error due to edge effect starts to show after 900 pixels on x-axis when the finger starts leaving the screen (b) After correction. The error starts appearing now from 1000 pixels onwards (figure is best viewed in color).

\subsection{2-D Gaussian Subpixel Interpolator}

Since the recovered capacitive signal of a complete finger can be modeled as a Gaussian, the most natural and straightforward interpolation method is Gaussian fitting which works by minimizing the energy function $\|\tilde{\mathbf{C}}(x, y)-\mathbf{C}(x, y: u, v, \sigma)\|_{2}^{2}$, where $\tilde{\mathbf{C}}$ is the recovered signal of one finger and $\mathbf{C}$ is the truncated version of the signal model given in (4.35). Both $\mathbf{C}$ and $\tilde{\mathbf{C}}$ are of the same size. 
The minimization of the energy function can be realized by an iterative optimization process, and is able to achieve very high accuracy. Due to high computation cost, it cannot be deemed suitable for a low memory touch controller implementation.

However, the capacitive touch application provides us with a unique opportunity of utilizing the high accuracy of Gaussian fitting without sacrificing speed. It is already known that in most cases a finger covers roughly $3 \times 3$ capacitors in both dimensions. We can therefore use a closed form solution of Gaussian fitting which was proposed in [123] for particle image velocimetry. Titled as 2-D Gaussian subpixel interpolator, it utilizes a $3 \times 3$ window around the peak location for an accurate and efficient subpixel peak detection. Here we demonstrate that the interpolator can be used for capacitive touch application and show through simulations that it is a more viable solution than any $2 \times 1-\mathrm{D}$ interpolator. We give a brief summary of this interpolator and the details can be found from [123]. A $3 \times 3$ window on the peak location $\tilde{c}$ at $x_{i}, y_{i}$ gives the $x$ and $y$ coordinates needed for the interpolator equations, which can be written as a matrix $G$ :

$$
G=\left[\begin{array}{ccc}
x_{i-1}, y_{i-1} & x_{i-1}, y_{i} x_{i-1}, y_{i+1} \\
x_{i}, y_{i-1} & x_{i}, y_{i} & x_{i}, y_{i+1} \\
x_{i+1}, y_{i-1} & x_{i+1}, y_{i} x_{i+1}, y_{i+1}
\end{array}\right]
$$

The subpixel displacement can then be derived as follows:

$$
\begin{aligned}
& \Delta x=\frac{w_{11} w_{01}-2 w_{10} w_{02}}{4 w_{20} w_{02}-w_{11}^{2}}, \\
& \Delta y=\frac{w_{11} w_{10}-2 w_{01} w_{20}}{4 w_{20} w_{02}-w_{11}^{2}},
\end{aligned}
$$

where the $w$ coefficients can be calculated as:

$$
w_{10}=\frac{1}{6} \sum_{i ; j=-1}^{1} i \ln \left(\tilde{c}_{x+i ; y+j}\right)
$$




$$
\begin{aligned}
& w_{01}=\frac{1}{6} \sum_{i ; j=-1}^{1} j \ln \left(\tilde{c}_{x+i ; y+j}\right) \\
& w_{11}=\frac{1}{4} \sum_{i ; j=-1}^{1} i j \ln \left(\tilde{c}_{x+i ; y+j}\right) \\
& w_{20}=\frac{1}{6} \sum_{i ; j=-1}^{1}\left(3 i^{2}-2\right) \ln \left(\tilde{c}_{x+i ; y+j}\right) \\
& w_{02}=\frac{1}{6} \sum_{i ; j=-1}^{1}\left(3 j^{2}-2\right) \ln \left(\tilde{c}_{x+i ; y+j}\right) \\
& w_{00}=\frac{1}{9} \sum_{i ; j=-1}^{1}\left(5-3 j^{2}-3 i^{2}\right) \ln \left(\tilde{c}_{x+i ; y+j}\right) .
\end{aligned}
$$

Once we have $\Delta x$ and $\Delta y$, the super-resolution finger peak location can be calculated using:

$$
\begin{aligned}
& c_{x}=\hat{c}_{x}+\Delta x \\
& c_{y}=\hat{c}_{y}+\Delta y
\end{aligned}
$$

where $\hat{c}_{x}, \hat{c}_{y}$ are the coarse resolution peak coordinates, or the sensor readings after charge transfer and signal recovery step.

\subsection{Simulations and Comparison}

In order to compare $2 \times 1$-D and 2 -D interpolators, a small portion of the touch panel containing one finger is used. The finger position is not changed throughout the experiments and noise is added in order to rigorously test the interpolators' performance. After calculating the subpixel peak location for each input SNR value, the mean error $E$ is calculated by measuring the Euclidean distance between the original noise free finger peak $\left(c_{x}, c_{y}\right)$ and the recovered super-resolution signal peak 
$\left(\beta_{x}, \beta_{y}\right)$ as:

$$
E=\sqrt{\left(c_{x}-\beta_{x}\right)^{2}+\left(c_{y}-\beta_{y}\right)^{2}}
$$

The accuracy and speed comparison between $2 \times 1$-D Gaussian and 2-D Gaussian interpolators is shown in Figures 5.14 and 5.15 respectively.

It can be seen that 2 -D interpolator is more accurate than $2 \times 1$-D Gaussian interpolator without any significant computation cost increase. Although the location error increases manifold, as the finger pitch angle decreases from 90, the 2-D Gaussian is still more accurate. The computation time difference between $2 \times 1-\mathrm{D}$ and 2-D Gaussian interpolator is in the order of $10^{-5}$ seconds.

By analyzing these results, we can state that a 2-D Gaussian interpolator will be an optimal choice for achieving subpixel finger location tracking on capacitive touch panels due to its high speed and accuracy.

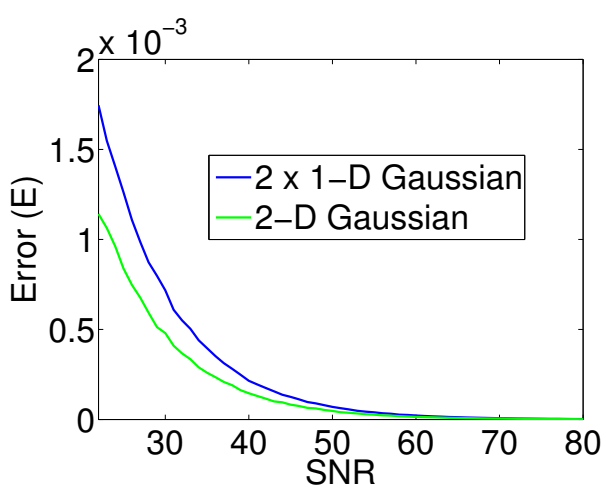

(a) Finger pitch angle $=90^{\circ}$

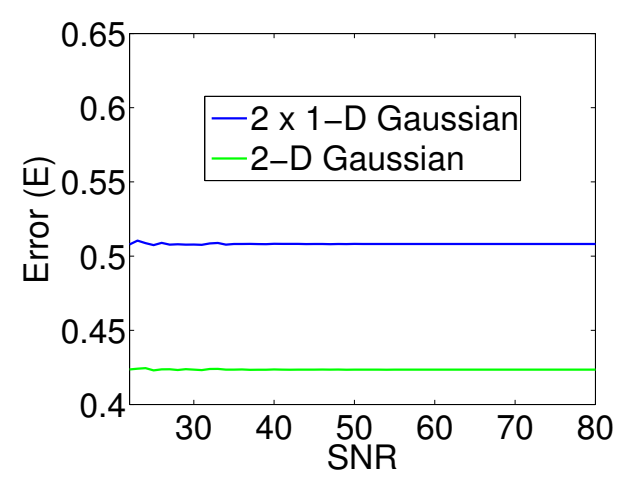

(b) Finger pitch angle $=15^{\circ}$

Figure 5.14: Subpixel error $E$ versus SNR for a steady single finger placed on a touch panel. Figure is best viewed in color.

\subsection{Validation of Research Findings}

The resolution obtained by algorithms proposed in Chapter 4 is called coarseresolution in academic literature. This resolution may not be sufficient for some high precision applications. The coarse resolution is limited by the distance between 


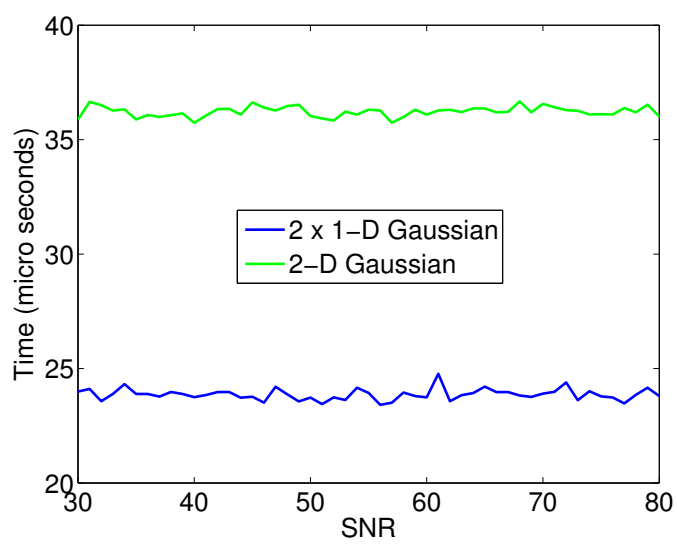

Figure 5.15: Computation time ( $\mu$ sec) vs SNR for a steady single finger placed on a touch panel. Figure is best viewed in color.

two capacitors on the panel. The coarse resolution can be improved by designing smaller electrodes. This technique needs modification in the hardware but it does not necessarily guarantee that the accuracy will be improved. In this Chapter, we have explored the problem of resolution enhancement for capacitive touch panels. We are treating the problem as an academic research problem and based on our studies we realize that the super-resolution problem can be computationally very costly. The aim is to present a rather simple algorithm to run in the real-time.

After exhaustive literature review and studying the signal characteristics of a finger touch signal, the technique selected for resolution enhancement was subpixel interpolation. During the review of academic research and white paper publications during the time of this research, it was noted that linear and centre of mass (centroid) sub pixel interpolators are the state-of-the-art in this field [122, 124]. Knowing that, we expanded our literature review outside our field of study and found out that in Particle Image Velocimetry (PIV), researchers have explored other sub-pixel interpolators for their field [123]. Utilizing the 2x1-D, parabolic and 2-D Gaussian interpolator from PIV to capacitive touch technology and showing via simulations that 2-D interpolator is most effective for this domain is the novel contribution of our work. The results have been validated in Chapter 5 via theoretical analysis in Section 5.3.1, and also via simulation in Matlab. The results presented in Chapter 5 are consistent with results obtained in subpixel interpolation studies in PIV 
$[123,125]$.

\subsection{Conclusion and Future Work}

At the end, we wish to reiterate the conclusions that we have drawn from the last three chapters. We tested various sensor designs in Matlab and hailed interleaved pattern as the most suitable for finger detection and tracking. We then moved on to sampling and proposed four algorithms out of which BMPNILS was the most accurate and CSAIM was the most efficient. In Chapter 5, we proposed that 2D Gaussian interpolator is more accurate than closed form $2 \times 1-\mathrm{D}$ interpolators. Putting these conclusions together, the full framework for a capacitive touch panel workings can be summarized using a flow chart diagram as shown in Figure 5.16.

This chapter serves as an insight into subpixel interpolation algorithms and their advantages in capacitive touch sensing. It is widely thought that three point $2 \times$ 1-D subpixel interpolation algorithms are used in existing touch sensing processors, though of course due to commercial sensitivity, such details are not always published. We showed new models for a finger touch response with varying pitch angle, and tested various 1-D and 2-D sub-pixel interpolation algorithms. We looked at interpolation bias, touch panel edge effect on accuracy and in the end designed a complete framework for capacitive touch sensing. Using our framework, one can design more accurate and efficient large scale capacitive touch panels. 


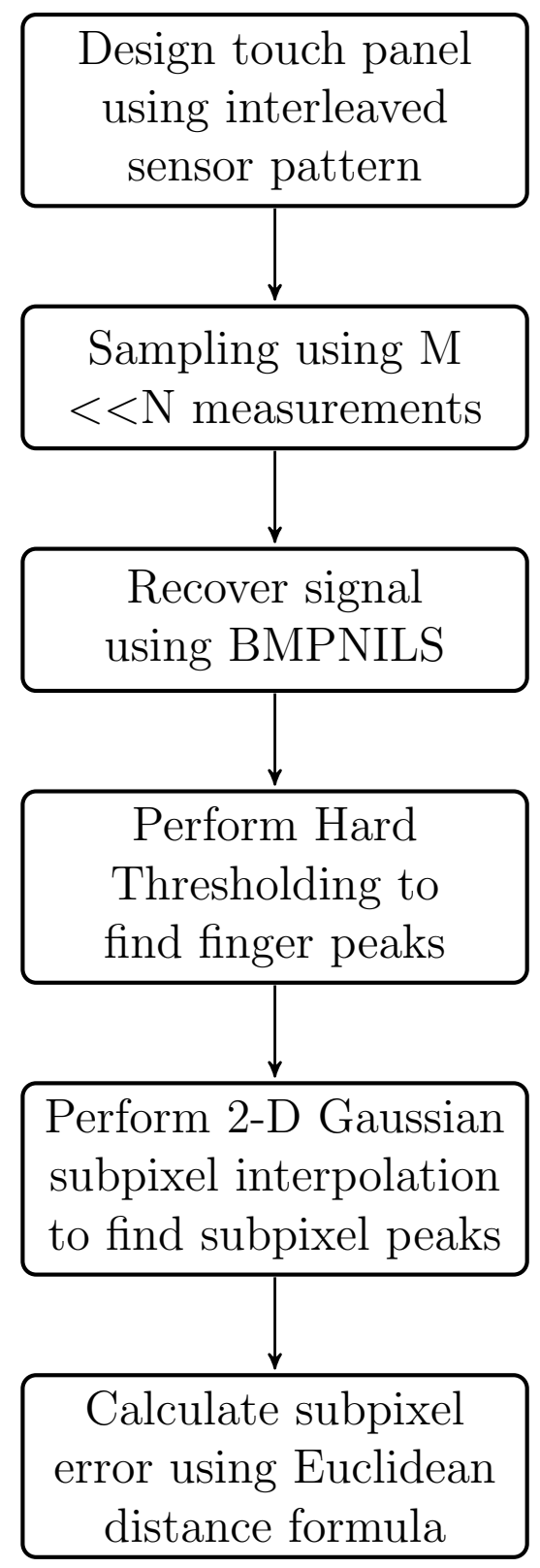

Figure 5.16: Framework depicting the recovery of noisy capacitive touch signals using BMPNILS and subpixel peak estimation using Gaussian interpolator. 


\section{Chapter 6}

\section{Summary and Outlook}

\subsection{Summary}

Large-scale touch panels are becoming popular in consumer industry as a means to disseminate knowledge. This thesis addressed several problems specific to capacitive touch technology and how it is being adapted to support large-scale panels.

\subsubsection{Motivation and Objectives}

We started by giving a strong motivation for the use of capacitive touch panels in retail and consumer industry, and how useful they can be when compared with the dominant infrared technology. We demonstrated a geo-visualization application designed for information display and gave reasoning of how touch panels can be useful in an educational and research environment. We went through pros and cons of all the prominent touch technologies and concluded that capacitive touch is better suited for the job. To give a background and flow to our work in this thesis, we presented a short historical review of developments in capacitive touch over the years, highlighting the major milestones in this field. Capacitive touch technology has been around for more than 40 years now but it is only recent that it is being considered as a viable option in large-scale touch panel manufacturing industry. Our 
detailed analysis of this technology provided us with three research problems that we decided to address in our research.

\subsubsection{Sensor Pattern Design and Testing}

After studying patented sensor patterns proposed for capacitive touch panels, it was obvious that the academic literature does not provide a methodology for comparing these patterns. Hence, in Chapter 3, we proposed a methodology for sensor patterns by testing and comparing six designs using a simulation environment. We placed a 2-D Gaussian shaped finger on a touch panel designed like a binary image and performed various movements including diagonal, circular and angle sweep to calculate mean tracking error using COM interpolator. We compared and contrasted the results of patterns amongst each other. It was found that the interleaved pattern performed better than the rest. The methodology presented can be extended to any sensor pattern design. We performed the diagonal movement experiment in hardware, using a robotic finger (capacitive stylus) and a motion stage. The mean tracking error was found out to be around $1 \mathrm{~mm}$. Fourier transform of patterns were studied and a hybrid pattern was proposed combining Fourier magnitude and phase from two different patterns. Being able to treat capacitive touch sensor patterns as images can help in designing hybrid patterns which improved performance by utilizing various image processing techniques. Doing simple geometric changes to an existing pattern also helps to increase the sensitivity of the capacitor. Sensitivity of sensor designs was also studied using ANSYS MOM solver and it was concluded that sensitivity and tracking accuracy depend on different aspects of the sensor design. The validation of methodology and results is also discussed in Section 3.6.

\subsubsection{Efficient and Accurate Sampling}

Perfecting a sensor design is just the first step. As the capacitive touch panel size increases, sampling becomes an issue because of the large number of sensors 
embedded on a panel. In Chapter 4, our objective was to minimize the sampling time for large-scale touch panels. We already knew that sampling time reduction is proportional to power reduction which is an area of interest while designing large panels. We opted for compressive sensing techniques and proposed four different algorithms for reduced sampling time. First, we designed structured binary matrices and used them for efficient sampling. Compressive sensing for Augmented Identity Matrices (CSAIM) was designed to quickly sample panels which use a stylus for input whereas Block Compressive Sensing using Binary Matrices (BCSBM) was designed for finger detection in low noise conditions on the panel with an assumption of finger covering more than one sensor. We learned that these algorithms underperformed in the presence of high noise, and thus more robust algorithms for noisy conditions were required. Going back to compressive sensing and greedy algorithms, we proposed Gramian Matching Pursuit (GMP), a simple variation of Matching Pursuit (MP) which utilizes Hadamard matrix as its sampling matrix. Because of the structured nature of Hadamard, the algorithm is much faster than traditional MP. We looked into the characteristics of capacitive touch signal and found them to be very unique. This insight inspired us to propose our final algorithm, Block Matching Pursuit with Non-Iterative Least Squares (BMPNILS), which utilizes those characteristics to perform efficient sampling with high precision even in noisy conditions. We compared these algorithms with each other and with other block sparsity based greedy algorithms in terms of accuracy and efficiency. It was found that BMPNILS is the most accurate of the algorithms proposed and is at par with other state of the art algorithms, whereas our structured matrix algorithms are the most efficient in terms of FLOPS. In the end, we performed an implementation analysis by calculating number of multiplication operations required for each algorithm. The proposed algorithm results were validated by comparison with state-of-the-art as discussed in Section 4.5. 


\subsubsection{Subpixel Interpolation}

After sampling, the final step for finger location detection is the interpolation. Traditional subpixel interpolation techniques used by industry are one-dimensional and can be improved in terms of accuracy. We tested four different 1-D three point interpolators by repeating the finger tracking simulation previously done in Chapter 3 and it was seen that Gaussian interpolator outperformed COM, linear and parabolic estimators. We checked the estimator bias on vertical and horizontal movements and proposed a simple two point interpolator for finger-near-edge cases. We utilized the 2-D Gaussian interpolator from Particle Image Velocimetry (PIV) and compared it with 1-D Gaussian and COM only to find that it performs better than those two and is very simple to implement in a low memory touch controller. We finished the chapter by listing a complete framework for capacitive touch panel design, sampling and interpolation based on the results from Chapters 3-5. The proposed methodology and results were validated by comparison with state-of-the-art as discussed in Section 5.5.

\subsection{Future Work}

In this thesis, we did research on various aspects of capacitive touch panels. New sampling algorithms, a methodology for testing sensor patterns and accurate subpixel interpolation open up the potential of future research in this area. Our work on sensor design testing methodology was the first of its kind in academic literature. Now we are seeing other researchers following suit and publishing their work in this area as mentioned at the end of Chapter 3. Similarly, we hope that our work on sampling and interpolation will also motivate researchers to develop algorithms that target large-scale touch panels. Fully utilizing the characteristics of capacitive touch signals to propose an efficient sampling framework is a novel contribution in this thesis. Many other variations of different greedy algorithms can now be explored for touch sampling. Let us list down some proposed future work for this research. 


\subsubsection{Sampling Matrix Training}

One of the straightforward extensions of our work is to further explore the area of sampling matrix/dictionary design directed for capacitive touch panels. From literature, we already know that a trained dictionary can help decrease the RMSE of the block sparse greedy algorithms $[126,127,128]$. However, based on our preliminary results, using Block K-SVD algorithm (reported to decrease recovery error for block sparse representations [126]) does not necessarily improve the reconstruction accuracy in our case. This can be seen in Figure 6.1, where an image representing two fingers resting on a touch panel was used as a test image for dictionaries designed using K-SVD and Block K-SVD algorithms. The figure shows that the improvement in PNSR is negligible even after training the dictionary using K-SVD and Block K-SVD. Initial analyses point out to the fact that because the image is too simple with few discontinuities, training does not have an effect on the reconstruction accuracy. However, it is too early to form a conclusion and we will be studying this phenomenon in the future.

\subsubsection{Image Processing Techniques}

We proposed treating capacitive touch panels as binary images in order to open them up for image processing techniques. This is just the tip of the iceberg. We know that by studying Fourier phase of the sensor image, we can hope to better understand what factors govern its performance. The future work will include understanding unwrapped Fourier phase and how its linearity (or non-linearity) effects the performance of the pattern. We already know that fringing fields contribute to the sensitivity of the pattern, but a complete and concrete procedure for improving fringing fields in a capacitive touch panel is still missing. There is a lot of potential for future research in this field. 


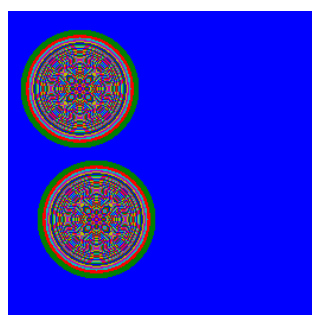

(a) Original image
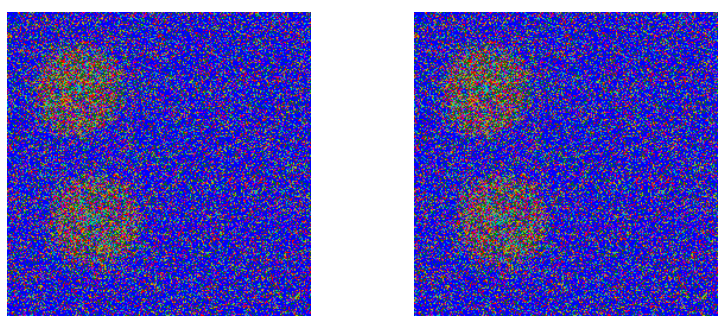

(b) Noisy image, PSNR: $27.5843 \mathrm{~dB}$

(c) Recovered using DCT dictionary, PSNR: 27.5843 $\mathrm{dB}$

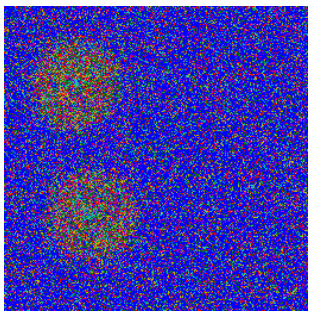

(d) Recovered by dictio- (e) Recovered by dictionary trained by K-SVD, nary trained by Block KPSNR: 27.6203 dB SVD, PSNR: $27.6645 \mathrm{~dB}$

Figure 6.1: Recovery of noisy capacitive touch panel image using dictionaries trained by K-SVD and Block K-SVD and comparison of their PSNR with image recovered using a Discrete Cosine Transform (DCT) dictionary.

\subsubsection{Accurate Noise Modeling}

We have discussed a number of interpolation algorithms that are normally used for subpixel interpolation. Whether it is the sampling or the interpolation algorithms, robustness against noise always plays a huge role in determining their performance. Researchers have primarily focused on improving noise reduction algorithms at the analog front-end of the touch measurement and tracking system, and we would like to see more work on adding noise filters at the digital back-end of the touch controller. We mentioned the charger and LCD noise in our thesis but these are just two major sources of noise and there are numerous others due to environmental factors like humidity, temperature and pressure. Due to these random sources, it is very difficult to construct an accurate noise model for capacitive touch screens. Also there are no 
standardized tests for assessing LCD or charger noise performance. Coming up with a way to characterize touch panel noise is not only an interesting area to work on, but also important in advancing the field of capacitive touch technology research.

\subsection{Final Thoughts}

I wish to conclude this thesis with a few personal thoughts on my work. It is foreseen that with the potential that capacitive touch technology holds, it will surpass infrared and become the market leader in the years to come. Millions of units are going to be sold and people will find capacitive touch panels to be essential in areas where mouse and keyboard based input systems are impractical. Some examples of such areas are shopping malls, hospitals and industrial control rooms. I do not believe that mouse and keyboard systems are going to be replaced by smallish touch screen in office setting, hence my thinking that the research on touch technology will focus solely on large-scale panels in the coming years.

When I started working on my $\mathrm{PhD}$, the amount of academic literature available concerned with the questions that we stated was close to none. This posed a significant challenge for us but it was also a blessing in disguise as now we are observing that people in academia are also giving thought to this field and publishing their findings. I hold a firm belief that in the coming years, the gap between industry and academia working on touch technology will close and there will be more work published on touch sampling algorithms, noise and finger modeling and subpixel interpolation for touch panels. Recently, prediction algorithms such as Kalman filter have been proposed for touch panels [129], and this all in all, is a truly exciting time to be working in this area.

Finally, when the touch panel hardware is perfected, the next challenge would be to develop such software applications which can make use of the extra precision provided by the capacitive touch technology. As I was working on EOS-GeoTouch system, I realized that the aesthetics for a touch panel software application differs 
widely from that of a mobile phone or tablet application. A highly precise touch panel is as good as the application user interface that it is displaying. It is a challenging task to bring the quality of software written for large-scale panel at the level of mobile applications, but as we all very well know, taking on challenging tasks and completing them, is in fact, the beauty of research. 


\section{Bibliography}

[1] Technavio, "Global Touchscreen Market Survey 2015-2019," http://www. technavio.com/report/global-touchscreen-market-2015-2019, [Online].

[2] G. Walker, "Fundamentals of Touch Technologies and Applications," Society for Information Display, 2011.

[3] — - Touch Displays, Handbook of Digital Imaging. John Wiley \& Sons, Ltd, 2015.

[4] E. A. Johnson, "Touch Display? a Novel Input/Output Device for Computers," Electronics Letters, vol. 8, no. 1, pp. 219-220, 1965.

[5] N. K. Mada and H. Jagadish, "Waking Up a Capacitive Touch-Sensing Device with an MCU Peripheral," Microchip, July 2011, www.eetimes.com/ document.asp?doc_id=1278965.

[6] "Peau Productions-Touch Screen Manufacturers Webpage," https://www. peauproductions.com/, [Online].

[7] G. Walker, "A Review of Technologies for Sensing Contact Location on the Surface of a Display," Journal of the Society for Information Display, vol. 20, no. 8, pp. 413-440, 2012.

[8] "Advanced Touch Sense Control from Infineon; a Technical Introduction," http://bit.ly/2fcM9SL, [Online]. 
[9] R. Seguine et al., "Capacitive Sensing Techniques and Considerations, The Basics," Design line, December, vol. 3, 2007.

[10] R. Fisher and D. Naidu, "A Comparison of Algorithms for Subpixel Peak Detection," in Image technology. Springer, 1996, pp. 385-404.

[11] "Microsoft Surface hub large scale touch panel," https://www.microsoft.com/ microsoft-surface-hub/en-sg/product-specs, [Online].

[12] C. Ian and M. Andrew, "Zytronic: Advances in PCap Touch Control Technology," http://zytronic.co.uk/news/ advances-in-p-cap-touch-control-technology/, [Online].

[13] "Stantum Resistive Touch Screen," http://www.stantum.com/, [Online].

[14] "Touchco resistive touch screen technology," http://www.touchco.com/, [Online].

[15] M. Fihn and R. Phares, Introduction to Touchscreen Technologies, Handbook of Visual Display Technology. Springer International Publishing, 2016.

[16] "elo Touch touchnology," https://www.elotouch.com/, [Online].

[17] "elo Touch eSAW technology," http://www.marketwired.com/press-release/ elo-introduces-new-esaw-touchscreen-technology-2124328.htm, [Online].

[18] J. Newton, "Touch Panel Display System," Feb. 10 2004, US Patent 6,690,363.

[19] "PQ Labs infrared panels," http://multitouch.com/, [Online].

[20] "Zaag Tech infrared panels," http://www.zaagtech.com/, [Online].

[21] "Citron infrared panels," http://www.citron.de/, [Online].

[22] E. A. Johnson, "Touch Displays: a Programmed Man-Machine Interface," Ergonomics, vol. 10, no. 2, pp. 271-277, 1967.

[23] "Apple iPhone," http://www.apple.com/iphone/, [Online]. 
[24] "Microsoft Surface," http://www.microsoft.com/Surface/Index.html, [Online].

[25] H. Gu and S. Chris, "Capacitive Touch Hardware Design Guide," MSP430, Texas Instruments, 2013.

[26] G. Barrett and R. Omote, "Projected-Capacitive Touch Technology," Information Display, vol. 26, no. 3, pp. 16-21, 2010.

[27] H. Akhtar and R. Kakarala, "A Methodology for Evaluating Accuracy of Capacitive Touch Sensing Grid Patterns," Journal of Display Technology, vol. 10, no. 8, pp. 672-682, 2014.

[28] — , "A Comparative Analysis of Capacitive Touch Panel Grid Designs and Interpolation Methods," in 2014 IEEE International Conference on Image Processing (ICIP). IEEE, 2014, pp. 5796-5800.

[29] —, "Efficient Capacitive Touch Sensing using Structured Matrices," in SPIE/ISET Electronic Imaging. International Society for Optics and Photonics, 2015, pp. 94-106.

[30] H. Akhtar and Q. Kemao, "An Accurate and Efficient Sampling Algorithm for Capacitive Touch Panels," in 2016 IEEE International Instrumentation and Measurement Technology Conference Proceedings. IEEE, 2016, pp. 1-6.

[31] E. E. Abdul Rahim, "Exploring a Desktop Virtual Reality Application for Education: The Perspectives of Spatial Knowledge Acquisition and Information Integration," Ph.D. dissertation, Lincoln University, 2013.

[32] J. W. Crampton, "Interactivity Types in Geographic Visualization," Cartography and Geographic Information Science, vol. 29, no. 2, pp. 85-98, 2002.

[33] D. DiBiase, "Rethinking Laboratory Education for an Introductory Course on Geographic Information," Cartographica: The International Journal for Geographic Information and Geovisualization, vol. 33, no. 4, pp. 66-72, 1996. 
[34] J. Dykes, A. MacEachren, and M. Kraak, "Facilitating Interaction for Geovisualization," Exploring geovisualization, pp. 265-291, 2005.

[35] A. Giordano and L. Wisniewski, "Teaching Cartography on the Web with a Multimedia GIS: A New Solution," in International Perspectives on Maps and the Internet. Springer, 2008, pp. 219-238.

[36] A. Kereszturi and D. Hyder, "Planetary Science in Higher Education: Ideas and Experiences," Journal of Geography in Higher Education, vol. 36, no. 4, pp. 499-525, 2012.

[37] J. B. Krygier, C. Reeves, D. DiBiase, and J. Cupp, "Design, Implementation and Evaluation of Multimedia Resources for Geography and Earth Science Education," Journal of Geography in Higher Education, vol. 21, no. 1, pp. 17-39, 1997.

[38] W. J. Lloyd, "Integrating GIS into the Undergraduate Learning Environment," Journal of Geography, vol. 100, no. 5, pp. 158-163, 2001.

[39] S. L. Marsh, "Using and Evaluating HCI Techniques in Geovisualization: Applying Standard and Adapted," Information Science, 2007.

[40] J. Halatsch and A. Kunze, "Value Lab: Collaboration in Space," in 11th International Conference on Information Visualization, 200\%. IV'0\%. IEEE, 2007, pp. 376-381.

[41] M. Damon, M. C. Kameyama, M. Knox, D. H. Porter, D. Yuen, and E. O. Sevre, "Interactive Visualization of 3D Mantle Convection," Visual Geosciences, vol. 13, no. 1, pp. 49-57, 2008.

[42] T. Ruth, S. Audersch, L. Huber, U. F. von Lukas, and J. Zabel, "Using DirectTouch Interaction for the Visual Exploration of Profiling Sensor Data," in OCEANS 2015-Genova. IEEE, 2015, pp. 1-8. 
[43] H. Guo, X. Fan, and C. Wang, "A Digital Earth Prototype System: DEPS/CAS," International Journal of Digital Earth, vol. 2, no. 1, pp. 3-15, 2009.

[44] R. M. Tamim, R. M. Bernard, E. Borokhovski, P. C. Abrami, and R. F. Schmid, "What Forty Years of Research says about the Impact of Technology on Learning a Second-Order Meta-Analysis and Validation Study," Review of Educational research, vol. 81, no. 1, pp. 4-28, 2011.

[45] R. M. Bernard, E. Borokhovski, R. F. Schmid, R. M. Tamim, and P. C. Abrami, "A Meta-Analysis of Blended Learning and Technology use in Higher Education: from the General to the Applied," Journal of Computing in Higher Education, vol. 26, no. 1, pp. 87-122, 2014.

[46] C. Hill, C. Corbett, and A. St Rose, Why so few? Women in Science, Technology, Engineering, and Mathematics,. Washington, DC: American Association of University Women, 2010.

[47] N. Orion, D. Ben-Chaim, and Y. Kali, "Relationship Between Earth-Science Education and Spatial Visualization," Journal of Geoscience Education, vol. 45, pp. 129-132, 1997.

[48] I. V. McLoughlin, L. M. Ang, and W. B. Goh, "Multi-touch Wall Displays for Informational and Interactive Collaborative Space," in Digital Urban Modeling and Simulation. Springer, 2012, pp. 318-338.

[49] "Environmental Systems Research Institute," http://www.esri.com, [Online].

[50] "PQ Labs Multi-Touch G4 Series Panel Data Sheet," http://www.pqlabs. com/download/datasheet/G4_Datasheet.pdf, [Online].

[51] "Microsoft PPI Multi-Touch Device Techspecs," https://www.microsoft.com/ microsoft-surface-hub/en-us/product-specs\#tech-specs, [Online]. 
[52] J. A. Harley, "Capacitive Touchscreen or Touchpad for Finger or Stylus," Jul. 31 2008, US Patent App. 12/183,456.

[53] B. L. Mackey, "Sensor Patterns for a Capacitive Sensing Apparatus," Oct. 31 2006, US Patent 7,129,935.

[54] P. T. Krein and R. D. Meadows, "The Electroquasistatics of the Capacitive Touch Panel," IEEE Transactions on Industry Applications, vol. 26, no. 3, pp. 529-534, 1990.

[55] J. Y. Ruan, P. C. P. Chao, and W. D. Chen, "A Multi-Touch Interface Circuit for a Large-Sized Capacitive Touch Panel," in IEEE Sensors, 2010. IEEE, 2010, pp. 309-314.

[56] Y. H. Tai, H. L. Chiu, and L. S. Chou, "Large-Area Capacitive Active Touch Panel Using the Method of Pulse Overlapping Detection," Journal of Display Technology, vol. 9, no. 3, pp. 170-175, 2013.

[57] C. L. Lin, C. S. Li, Y. M. Chang, T. C. Lin, J. F. Chen, and U. C. Lin, "Pressure Sensitive Stylus and Algorithm for Touchscreen Panel," Journal of Display Technology, vol. 9, no. 1, pp. 17-23, 2013.

[58] Z. Baharav and R. Kakarala, "Capacitive Touch Sensing: Signal and Image Processing Algorithms," in ISET/SPIE Electronic Imaging. International Society for Optics and Photonics, 2011.

[59] A. A. Tareev, "Touchpad with Interleaved Traces," Nov. 14 2000, US Patent $6,147,680$.

[60] M. Nagarajan and K. A. Ningrat, "ITO Pattern for Capacitive Touchscreen Applications," Aug. 6 2012, US Patent App. 13/567,855.

[61] M. Trend, S. Brunet, and E. Yilmaz, "Electrode Layout for Touch Screens," Feb. 3 2011, US Patent App. 12/534,748. 
[62] C. Y. Chang and S. T. Chien, "Conductor Pattern Structure of Capacitive Touch Panel," Jul. 10 2012, US Patent 8,217,902.

[63] J. Lukanc, S. L. Morein, C. A. Ludden, G. P. Semeraro, J. K. Reynolds, P. Shepelev, and T. Mackin, "Capacitive Sensing using a Matrix Electrode Pattern," Dec. 20 2013, US Patent App. 14/137,692.

[64] R. Ritter, K. Brewer, V. Souchkov, and S. Narasimhan, "Sensor Patterns for Mutual Capacitance Touchscreens," Jul. 7 2015, US Patent 9,075,484.

[65] R. Mohindra, "Identifying Hover and/or Palm Input and Rejecting Spurious Input for a Touch Panel," Jul. 14 2015, US Patent 9,081,450.

[66] J. T. Bernstein, D. Amm, O. S. Leung, C. T. Mullens, B. M. King, B. R. Land, and R. T. Cutler, "Touch and Hover Sensing," Mar. 28 2016, US Patent App. $15 / 083,102$.

[67] S. C. Kim, A. Israr, and I. Poupyrev, "Tactile Rendering of 3D Features on Touch Surfaces," in Proceedings of the 26th annual ACM symposium on User interface software and technology. ACM, 2013, pp. 531-538.

[68] H. W. Klein, "Noise immunity of touchscreen devices," Cypress Semiconductor Corporation, White Paper, 2013.

[69] "Microchip, PIC32 GUI Development Board with Projected Capacitive Touch, Part Number: DM320015," www.microchip.com/en560014, [Online].

[70] "Cypress Semiconductor, Universal CapSense Controller Board, Part Number: CY3280," http://www.cypress.com/documentation/development-kitsboards/ cy3280-24x94-universal-capsense-controller-board, [Online].

[71] "Newport, XPS Series Motion Controllers, Part Number: XPS-Q8," https: //www.newport.com/p/XPS-Q8, [Online].

[72] A. V. Oppenheim and J. S. Lim, "The importance of phase in signals," IEEE Proceedings, vol. 69, no. 5, pp. 529-541, 1981. 
[73] W. H. Grover, "Interdigitated Array Electrode Sensors: their Design, Efficiency, and Applications," MSc Thesis, 1999.

[74] J. Lee, M. T. Cole, J. C. S. Lai, and A. Nathan, "An Analysis of Electrode Patterns in Capacitive Touch Screen Panels," Journal of Display Technology, vol. 10, no. 5, pp. 362-366, 2014.

[75] B. Cannon and C. Brennan, "Electrostatic Simulation Methodology for Capacitive Touch-Screen Panels," in 25th IET Irish Signals 83 Systems Conference 2014 and 2014 China-Ireland International Conference on Information and Communications Technologies (ISSC 2014/CIICT 2014). IET, 2013, pp. 216-220.

[76] "COMSOL AC/DC Module," https://www.comsol.com/acdc-module, [Online].

[77] B. Li, T. Wei, X. Wei, J. Wang, W. Liu, and R. Zheng, "A touch prediction and window sensing strategy for low-power and low-cost capacitive multitouch screen systems," Journal of Display Technology, vol. 12, no. 6, pp. 646-657, 2016.

[78] M. G. A. Mohamed and H. Kim, "Concurrent driving method with fast scan rate for large mutual capacitance touch screens," Journal of Sensors, vol. 2015, 2015.

[79] C.-H. Huang and C.-Y. Chang, "An area and power efficient adder-based stepwise linear interpolation for digital signal processing," IEEE Transactions on Consumer Electronics, vol. 62, no. 1, pp. 69-75, 2016.

[80] M. Miyamoto, M. Hamaguchi, and A. Nagao, "A 143 x 81 Mutual-Capacitance touch-Sensing Analog Front-end with Parallel Drive and Differential Sensing Architecture," IEEE Journal of Solid-State Circuits, vol. 50, no. 1, pp. 335$343,2015$. 
[81] S. Ko, H. Shin, J. Lee, H. Jang, B. C. So, I. Yun, and K. Lee, "Low Noise Capacitive Sensor for Multi-Touch Mobile Handset's Applications," in IEEE Asian Solid State Circuits Conference (A-SSCC), 2010. IEEE, 2010, pp. 1-4.

[82] K. Lim, K. S. Jung, C. S. Jang, J. S. Baek, and I. B. Kang, "A Fast and Energy Efficient Single-Chip Touch Controller for Tablet Touch Applications," Journal of Display Technology, vol. 9, no. 7, pp. 520-526, 2013.

[83] C. Luo, M. Borkar, A. J. Redfern, J. H. McClellan et al., "Compressive Sensing for Sparse Touch Detection on Capacitive Touch Screens," IEEE Journal on Emerging and Selected Topics in Circuits and Systems, vol. 2, no. 3, pp. 639648, 2012.

[84] "MTCH6102 Low-Power Projected Capacitive Touch Controller," http:// ww1.microchip.com/downloads/en/DeviceDoc/40001750A.pdf, [Online].

[85] Y. Kim and A. H. Tewfik, "An Efficient Detection on Capacitive Touch Screens Using Bandwidth Expansion," in 6th International Symposium on Communications, Control and Signal Processing (ISCCSP). IEEE, 2014, pp. 534-537.

[86] — - "Low Power Detection on Capacitive Touch Screens," in Global Conference on Signal and Information Processing (GlobalSIP), 2013 IEEE. IEEE, 2013, pp. 638-641.

[87] — - "Low Energy and Latency Touch Detection using Group Testing," in 21st European Signal Processing Conference (EUSIPCO 2013). IEEE, 2013, pp. $1-5$.

[88] D. L. Donoho, "Compressed Sensing," IEEE Transactions on Information Theory, vol. 52, no. 4, pp. 1289-1306, 2006.

[89] E. J. Candès, J. Romberg, and T. Tao, "Robust Uncertainty Principles: Exact Signal Reconstruction from Highly Incomplete Frequency Information," IEEE Transactions on Information Theory, vol. 52, no. 2, pp. 489-509, 2006. 
[90] E. J. Candès et al., "Compressive Sampling," in Proceedings of the international congress of mathematicians, vol. 3. Madrid, Spain, 2006, pp. 14331452.

[91] E. J. Candès and M. B. Wakin, "An Introduction to Compressive Sampling," IEEE Signal Processing Magazine, vol. 25, no. 2, pp. 21-30, 2008.

[92] R. A. DeVore, "Deterministic Constructions of Compressed Sensing Matrices," Journal of Complexity, vol. 23, no. 4, pp. 918-925, 2007.

[93] H. Rauhut, "Circulant and Toeplitz matrices in Compressed Sensing," arXiv preprint arXiv:0902.4394, 2009.

[94] W. U. Bajwa, J. D. Haupt, G. M. Raz, S. J. Wright, and R. D. Nowak, "Toeplitz-Structured Compressed Sensing Matrices," in IEEE/SP 14th Workshop on Statistical Signal Processing, 2007, SSP'07. IEEE, 2007, pp. 294-298.

[95] M. A. Figueiredo, R. D. Nowak, and S. J. Wright, "Gradient Projection for Sparse Reconstruction: Application to Compressed Sensing and Other Inverse Problems," IEEE Journal of Selected Topics in Signal Processing, vol. 1, no. 4, pp. 586-597, 2007.

[96] S. G. Mallat and Z. Zhang, "Matching Pursuits with Time-Frequency Dictionaries," IEEE Transactions on Signal Processing, vol. 41, no. 12, pp. 33973415, 1993.

[97] Y. C. Pati, R. Rezaiifar, and P. Krishnaprasad, "Orthogonal Matching Pursuit: Recursive Function Approximation with Applications to Wavelet Decomposition," in Twenty-Seventh Asilomar Conference on Signals, Systems and Computers. IEEE, 1993, pp. 40-44.

[98] G. M. Davis, S. G. Mallat, and Z. Zhang, "Adaptive Time-Frequency Decompositions," Optical Engineering, vol. 33, no. 7, pp. 2183-2191, 1994. 
[99] J. Tropp, A. C. Gilbert et al., "Signal Recovery from Random Measurements via Orthogonal Matching Pursuit," IEEE Transactions on Information Theory, vol. 53, no. 12, pp. 4655-4666, 2007.

[100] D. Needell and J. A. Tropp, "CoSaMP: Iterative Signal Recovery from Incomplete and Inaccurate Samples," Applied and Computational Harmonic Analysis, vol. 26, no. 3, pp. 301-321, 2009.

[101] A. C. Gilbert, S. Guha, P. Indyk, S. Muthukrishnan, and M. Strauss, "Nearoptimal Sparse Fourier Representations via Sampling," in Proceedings of the thiry-fourth annual ACM symposium on Theory of computing. ACM, 2002, pp. $152-161$.

[102] A. C. Gilbert, M. J. Strauss, J. A. Tropp, and R. Vershynin, "Algorithmic Linear Dimension Reduction in the 11 Norm for Sparse Vectors," arXiv preprint cs/0608079, 2006.

[103] J. H. Jung and D. P. OLeary, "Implementing an Interior Point Method for Linear Programs on a CPU-GPU System," Electronic Transactions on Numerical Analysis, vol. 28, no. 174-189, p. 37, 2008.

[104] J. Lu, H. Zhang, and H. Meng, "Novel Hardware Architecture of Sparse Recovery Based on FPGAs," in 2nd International Conference on Signal Processing Systems (ICSPS), 2010, vol. 1. IEEE, 2010, pp. V1-302.

[105] S. Foucart and M.-J. Lai, "Sparsest Solutions of Underdetermined Linear Systems via lq-Minimization for $0<\mathrm{q}<1$," Applied and Computational Harmonic Analysis, vol. 26, no. 3, pp. 395-407, 2009.

[106] E. J. Candès, "The Restricted Isometry Property and its Implications for Compressed Sensing," Comptes Rendus Mathematique, vol. 346, no. 9, pp. 589-592, 2008. 
[107] R. Baraniuk, M. Davenport, R. DeVore, and M. Wakin, "A Simple Proof of the Restricted Isometry Property for Random Matrices," Constructive Approximation, vol. 28, no. 3, pp. 253-263, 2008.

[108] D. L. Donoho, "Neighborly polytopes and sparse solutions of underdetermined linear equations," 2005.

[109] M. Rudelson and R. Vershynin, "On sparse reconstruction from fourier and gaussian measurements," Communications on Pure and Applied Mathematics, vol. 61, no. 8, pp. 1025-1045, 2008.

[110] A. M. Tillmann and M. E. Pfetsch, "The Computational Complexity of the Restricted Isometry Property, the Nullspace Property, and Related Concepts in Compressed Sensing," IEEE Transactions on Information Theory, vol. 60, no. 2, pp. 1248-1259, 2014.

[111] K. J. Horadam, Hadamard Matrices and their Applications. Princeton university press, 2007.

[112] B. S. Kashin, "Diameters of some Finite-dimensional Sets and Classes of Smooth Functions," Izvestiya Rossiiskoi Akademii Nauk. Seriya Matematicheskaya, vol. 41, no. 2, pp. 334-351, 1977.

[113] A. Y. Garnaev and E. D. Gluskin, "The Widths of a Euclidean Ball," in Dokl. Akad. Nauk SSSR, vol. 277, no. 5, 1984, pp. 1048-1052.

[114] M. Yaghoobi, S. McLaughlin, and M. E. Davies, "Super-Resolution Sparse Projected Capacitive Multitouch Sensing," in Intelligent Signal Processing Conference 2013 (ISP 2013), IET. IET, 2013, pp. 1-6.

[115] Y. C. Eldar, P. Kuppinger, and H. Bolcskei, "Block-sparse Signals: Uncertainty Relations and Efficient Recovery," IEEE Transactions on Signal Processing, vol. 58, no. 6, pp. 3042-3054, 2010. 
[116] R. G. Baraniuk, V. Cevher, M. F. Duarte, and C. Hegde, "Model-based Compressive Sensing," IEEE Transactions on Information Theory, vol. 56, no. 4, pp. 1982-2001, 2010.

[117] A. Poorghanad, A. Sadr, and A. Kashanipour, "Generating High Quality Pseudo Random Number using Evolutionary Methods," in International Conference on Computational Intelligence and Security, vol. 1. IEEE, 2008, pp. 331-335.

[118] T. Blumensath and M. E. Davies, "Iterative Hard Thresholding for Compressed Sensing," Applied and Computational Harmonic Analysis, vol. 27, no. 3, pp. 265-274, 2009.

[119] MISRA-C, "Guidelines for the use of the C language in Critical Systems," http://www.misra.org.uk/, [Online].

[120] C. Holz and P. Baudisch, "The Generalized Perceived Input Point Model and how to Double Touch Accuracy by Extracting Fingerprints," in Proceedings of the SIGCHI Conference on Human Factors in Computing Systems. ACM, 2010, pp. 581-590.

[121] S. Rogers, J. Williamson, C. Stewart, and R. Murray-Smith, "AnglePose: Robust, Precise Capacitive Touch Tracking via 3d Orientation Estimation," in Proceedings of the SIGCHI Conference on Human Factors in Computing Systems. ACM, 2011, pp. 2575-2584.

[122] T. O Connor, "mTouch Projected Capacitive Touch Screen Sensing Theory of Operation," TB3064 Microchip Technology Inc, vol. 16, 2010.

[123] H. Nobach and M. Honkanen, "Two-Dimensional Gaussian Regression for SubPixel Displacement Estimation in Particle Image Velocimetry or Particle Position Estimation in Particle Tracking Velocimetry," Experiments in fluids, vol. 38, no. 4, pp. 511-515, 2005. 
[124] "PSoC 4 Capacitive Sensing (CapSense CSD), PSoC Creator Component Datasheet," http://www.cypress.com/file/127396/download, [Online].

[125] J. Westerweel, "Fundamentals of digital particle image velocimetry," Measurement science and technology, vol. 8, no. 12, p. 1379, 1997.

[126] L. Zelnik-Manor, K. Rosenblum, and Y. C. Eldar, "Dictionary Optimization for Block-Sparse Representations," IEEE Transactions on Signal Processing, vol. 60, no. 5, pp. 2386-2395, 2012.

[127] Y. Zhang, J. Liu, M. Li, and Z. Guo, "BSIK-SVD: A Dictionary-Learning Algorithm for Block-Sparse Representations," in 2014 IEEE International Conference on Acoustics, Speech and Signal Processing (ICASSP). IEEE, 2014, pp. $3528-3532$.

[128] L. Zelnik-Manor, K. Rosenblum, and Y. C. Eldar, "Sensing Matrix Optimization for Block-Sparse Decoding," IEEE Transactions on Signal Processing, vol. 59, no. 9, pp. 4300-4312, 2011.

[129] C.-L. Lin, Y.-M. Chang, C.-C. Hung, C.-D. Tu, and C.-Y. Chuang, "Position Estimation and Smooth Tracking with a Fuzzy-logic-based Adaptive Strong Tracking Kalman Filter for Capacitive Touch Panels," IEEE Transactions on Industrial Electronics, vol. 62, no. 8, pp. 5097-5108, 2015. 


\section{List of Publications}

[1] H. Akhtar, R. Kakarala, "A Methodology for Evaluating Accuracy of Capacitive Touch Sensing Grid Patterns," Display Technology, Journal of, vol. 10, no. 8, pp. 672-682, 2014.

[2] H. Akhtar, R. Kakarala, "A Comparative Analysis of Capacitive Touch Panel Grid Designs and Interpolation Methods," in 2014 IEEE International Conference on Image Processing (ICIP). Paris, 2014, pp. 5796-5800.

[3] H. Akhtar, R. Kakarala, "Efficient Capacitive Touch Sensing using Structured Matrices," in SPIE/ISET Electronic Imaging. International Society for Optics and Photonics, San-Fransisco, 2015, pp. 94-104.

[4] H. Akhtar, Q. Kemao, "An Accurate and Efficient Sampling Algorithm for Capacitive Touch Panels," in 2016 IEEE International Instrumentation and Measurement Technology Conference Proceedings. Taipei, 2016, pp. 1-6.

[5] H. Akhtar, Q. Kemao, and R. Kakarala. "A review of sensing technologies for small and large-scale touch panels," in Fifth International Conference on Optical and Photonics Engineering. International Society for Optics and Photonics, 2017. 


\section{List of Papers under review/ to be submitted}

[1] H. Akhtar, I. Kerlow, "EOS-GeoTouch: a Large-Scale Multi-Touch Geovisualization Portal for Earth Science Education and Outreach," Journal of Geography in Higher Education. (Revision submitted - July 2017)

[2] H. Akhtar, Q. Kemao, "Accurate and Efficient Super-Resolution Sampling for Capacitive Touch Panels," in Elsevier Displays. (Under Review)

[3] H. Akhtar, R. Kakarala, "A Compressive Sensing Framework for Capacitive Touch Technology using Structured Matrices," (Preparing For Submission) 